\title{
Volune V
}

Technical Assessment of Critical Issues

in the Steady State Operation of Fusion Confinement Devices

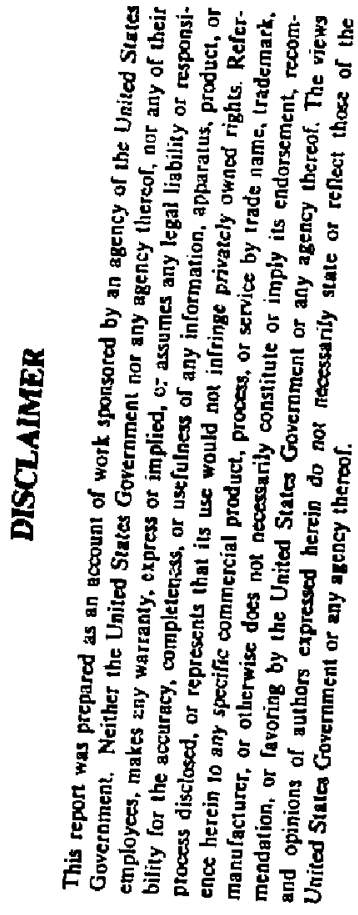

Panel Members

D.M. Goebel', Assessment Chairman

J.N. Downing 2

P.H. Edmonds 3,4

R. Puigh 5

D.K. Owens 6

M. Ulrickson 6

J.B. Whitley7

A.J. Wootton ${ }^{4}$

January 1988

${ }^{1}$ Institute for Plasma and Fusion Research, University of Califomia, Los Angeles, CA 90024

${ }^{2}$ Los Alamos National Laboratory, Los Alamos, NM 87545

3 Oak Ridge National Laboratory, Fusion Energy Division, Oak Ridge, TN 37830

${ }^{4}$ Fusion Research Center, University of Texas at Austin, Austin, TX 78712

5 HEDL, P.O. Box 1970, Richland, WA 99352

${ }^{6}$ Princeton Plasma Physics Laboratory, Princeton University, Princeton, NJ 08544

${ }^{7}$ Sandia National Laboratories, Albuquerque, NM 87185 


\section{Volume $V$}

\section{Technical Assessment of Critical Issues}

in the Steady State Operation of Fusion Confinement Devices

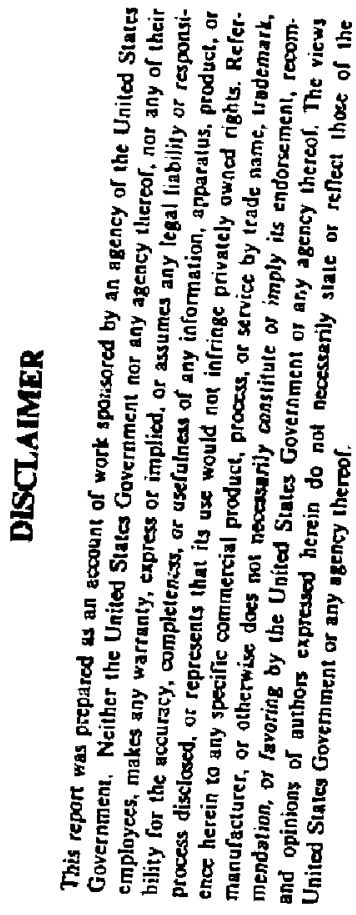

Panel Members

D,M. Goebel' , Assessment Chairman

J.N. Downing 2

P.H. Edmonds 3,4

R. Puigh5

D.K. Owens 6

M. Ulrickson6

J.B. Whitley 7

A.J. Wootton ${ }^{4}$

January 1988

1 Institute for Plasma and Fusion Research, University of Califomia, Los Angeles, CA 90024

2 Los Alarnos National Laboratory, Los Alamos, NM 87545

${ }^{3}$ Oak Ridge National Laboratory, Fusion Energy Division, Oak Ridge, 'TN 37830

${ }^{4}$ Fusion Research Center, University of Texas at Austin, Austin, TX 78712

5 HEDL, P.O. Box 1970, Richland, WA 99352

6 Princeton Plasma Physics Laboratory, Princeton University, Princeton, NJ 08544

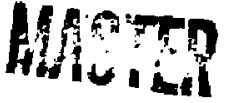

${ }^{7}$ Sandia National Laboratories, Albuquerque, NM 87185 


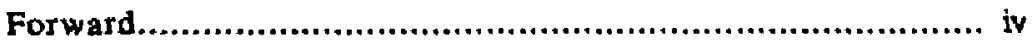

Executive Summary............................................................. vi

Part A : Physics Issues for Steady-State Operation.............................. A-1

Introduction................................................................ A-2

I. Equilibrium.......................................................... A

a) The tokamak..................................................... A-3

b) Stellarators, heliacs, and torsatrons............................... A-5

c) The reversed field pinch......................................... A-5

II. Stability and Profile Control............................................ A-6

III. Density Control....................................................... A-8

a) Particle sources.................................................. A-8

b) Recycling........................................................ A

c) Particle sinks................................................. A-10

IV. Impurity Control Requirements...................................... A-13

a) Divertor issues.................................................. A-13

b) Edge tempt ature control...................................... A-14

c) Flux modification.............................................. A-14

V. Burn Control........................................................... A5

V. Helium Ash Removal................................................. A-16

VII. Edge Plasma Conditions.............................................. A-18

VIII. Impurity Control Systems in Tokamaks.............................. A-19

a) Limiters and pump limiters......................................... A-19

b) Magnetic divertors............................................. A-22

c) Ergodized plasma edge for impurity control...................... A-23

Part B : Technology Issues for Steady-State Operation........................ B-1

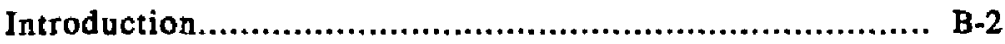

I. PJasms Facing Components....................................... B-3

a) Plasma operational R\&D issues................................ B-3

b) Materials R\&D issues.......................................... B-5 
I. Materials Issues for Steady State Devices............................ B-8

III. Thermal Stress and Fatigue.......................................... B-18

IV. Heat Transfer...................................................... B-25

V. Neutron Irradiation Effects............................................ B-31

a) Neutron irradiation effects issues................................ B-31

b) HHF/PMI materials............................................. B-31

c) Steady State Operation............................................ B-32

d) Irradiation temperatures............................................. B-32

e) Neutron irradiation effects....................................... B-32

f) Testing requirements............................................ B-34

g) Present status................................................... B-35

VI. Erosion and Redeposition............................................. B-36

a) Erosion data base................................................. B-37

b) Redeposition data base........................................... B-44

c) Conclusions for Steady State Operation........................... B-49

VII. Tritium Issues for Steady State Devices............................... B-53

a) Tritium inventory .............................................. B-53

b) Tritium permeation............................................... B-54

c) Laboratory experiments.......................................... B-55

VIII. Transient Behavior................................................... B-57

IX. Steady State Heating and Fueling Components Issues.............. B-59

a) Heating Systems.................................................. B-59

b) Fueling Systems................................................. B-6t

$X$. Diagnostics and Plasma Control.................................... B-62

a) Diagnostic system overview........................................ B-62

b) Specific diagnostic problems unique to steady state............. B-63

c) Recommendations............................................ B-71

XI. Maintenance in Steady State Devices................................. B-74

Part C : Steady State Issues in Altemative Devices.............................. C-1

I. Reversed Field Pinch................................................ C-2

a) Introduction........................................................ 
b) Plasma control.......................................... C-4

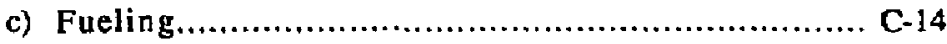

d) Impurity control......................................... C-15

e) Current Drive........................................... C-27

f) Heating......................................................

II. Spheromaks............................................. C-32

a) Spheromak characteristics............................. C-33

b) Prospect for steady-state operation............................. C-34

c) Spheromak steady-state technology issues...................... C-35

Part D : Devices and Facilities to Address Steady State Issues............... D-1

Introduction..................................................... D-2

I. Planned confinement devices before ETR........................ D-3

II. ETR/Tiber Design and R\&D Requirements....................... D-9

III. ATF Steady State.............................................. D-16

a) ATF schedule.............................................. D-16

b) Steady state program..................................... D-22

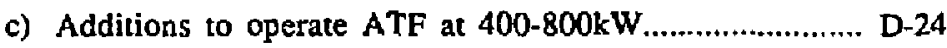

d) Additions to operate ATF at 2-4 MW........................ D-25

e) Power and particle handling.............................. D-25

IV. Large Helical System........................................... D-29

V. U.S. PMI/HHF Laboratory facilities............................... D-34

a) Plasma Materials Test Facility (PMTF)....................... D-35

b) Plasma Surface Interactions Facility (PISCES)................ D-39

c) Tritiurn Plasma Experiments (TPX)........................ D-43

d) Hot Cell Electron Beam Facility (HEBF)....................... D-45

e) ORNL PMI Facilities................................... D-47

f) Continuous Current Tokamak (CCT) ....................... D-48 


\section{Eorward}

Technical Assessment of Critical lssues

in the Steady State Operation of Fusion Confinement Devices

This is the fifth in a series of technical assessments of important issues produced by the Fusion Technologies Branch of the Division of Development and Technology in the Office of Fusion Energy (OFE). Included in the Fusion Technologies task area is work in PlasmaMaterials Interactions (PMI) and High Heat Flux Materials and Component Development (HFFMCD). The present technical assessment identifies the critical issues related to the steady state operation of an ETR or ITER device and emphasizes the critical areas in the PMI and HHFMCD fielcis because of their vital importance to the successful operation of steady state confinement devices. This assessment includes physics issues and information concerning steady state alternative devices.

The assessments produced by the Fusion Technologies Branch since 1984 include:

Volume I Critical Issues in the Plasma Materials Interaction Field

Volume II Critical Issues in the High Heat Flux Materials \& Component Development Field

Volume III Strategy for Intemational Collaborations in the Areas of PMI and HHFMCD

Volume IV Technical Assessment of Plasma-Interactive Options for Claddings and Attachments for Steady State

Volume V Critical Issues in the Steady State Operation of Fusion Confinement Devices

The above technical assessments have been, and new program plans in progress, are being prepared by task groups composed of persons from the various laboratories, private industry, and universities, which contribute to all aspects of the Magnetic Fusion Energy Program. The task groups cat across confinement concept lines. Each task group of six to ten principal investigators and/or consultants work under the guidance of a chairman drawn from a national laboratory or major university, and his counterpart, a staff member of the Fusion Technologies 
Branch. In the case of PMI and HHFMCD, the OFE counterpart is Dr. Marvin M. Cohen. Each assessment operates through a number of ad-hoc task groups which were charged with the problem definition and discussion for specific technical areas.

The emphasis of the present planning process is to seek out and examine potential problems early; determine the state of technical readiness, and to prioritize the research and development programs to meet the requirements which must be satisfied for the successful development of fusion reactors. It is important to realize that the assessments and plans describe problem areas, and that these assessments will have to be updated periodically. Furthermore, they should be regarded as outlining the major avenues to be explored, rather than as a detailed road map. The detailed approach to specific problems will be proposed by individual investigators in response to a forthcoming program plan.

A total of over 60 individuals have been involved in the various stages of the production of the technical assessments and program plans. The wide representation of national laboratories, universities, and industry was encouraged to remove institutional bias as much as possibie. I would like to thank all of the members of the task groups and technical community who contributed to these efforts.

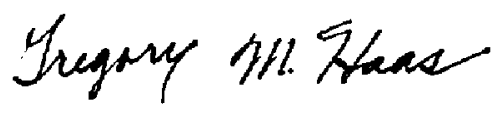

\author{
Gregory M. Haas, Chief \\ Fusion Technologies Branch \\ Division of Development and Technology \\ Office of Fusion Energy \\ Office of Energy Research \\ Department of Energy
}




\section{Executive Summary}

Critical issues for the steady state operation of plasma confinement devices exist in both the physics and technology fields of fusion research. Due to the wide range and number of these issues, this technical assessment has focused on the crucial issues associated with the plasma physics and the plasma interactive components. The document provides information on the problem areas that affect the design and operation of a steady state ETR or ITER type confinement device. It discusses both tokamaks and alternative concepts, and provides a survey of existing and planned confinement machines and laboratory facilities that can address the identified issues.

A universal definition of steady state operation is difficult to obtain. From a physics point of view, steady state is generally achieved when the time derivatives approach zero and the operation time greatly exceeds the characteristic time constants of the device. Steady state operation for materials depends on whether thermal stress, creep, fatigue, radiation damage, or power removal are being discussed. For erosion issues, the fluence and availability of the machine for continuous operation are important, assuming that transient events such as disruptions do not limit the component lifetimes. The panel suggests, in general ! arms, that steady state requires plasma operation from 100 to 1000 seconds and an availability of more than a few percent, which is similar to the expectations for an ETR type device.

The assessment of critical issues for steady stace operation is divided into four sections: 1) physics issues; 2) technology issues; 3) issues in alternative concepts; and 4) devices and laboratory facilities that can address these problems. The major technical issues in these groups ane:

\section{A. Physics Issues}

1. Non-inductive current drive. Current drive is needed to establish the steady state plasnta equilibrium in the generic machine families of tokamaks and Reversed Field Pinches (RFP). 
Only stellarator types of machines avoid this problem, at the expense of complicated magnetic coil configurations, assuming that internal currents that may develop will not need to be canceled by an externally produced opposite current. There are several candidates for producing current drive, but these have not demonstrated good efficiency at high density. A solution will have be achieved before steady state tokamaks and RFPs can be operated.

2. Profile evolution and stabilicy. Profile modification by external techniques, such as localized heating, fueling, or current drive, has resulted in improvements in confinement and plasma performance in many machines. Control of the profile in steady state may not be possible due to profile consistency or other factors in the plasma. The amount of profile control and the optimum techniques to produce the desired profile must be determined for steady state plasma operation with the desired improvements observed during profile modification in pulsed devices. A collaboration on the TORE SUPRA tokamak between the U.S. and France will investigate this issue during pellet injection and pump limiter exhaust experiments with near steady state conditions. Additional experiments are needed on long pulse machines such as JET and JT-60.

3. Impurity Control. Impurity production and accumulation are important issues for steady state machines. Control of the edge plasma temperatures and fluxes may lead to lower impurity source rates. Despite several candidate techniques for edge control, experiments have not established the degree of control to date. Impurity accumulation during $\mathrm{H}$-mode confinement has been observed in ASDEX, and techniques to avoid this problem must be developed.

4. Particle removal for density impurity and He-ash control. Divertors and pump limiters are the major candidates for providing particle removal for ETR. These devices have several common issues: a) Control of impurity production that contaminates the plasma; b) Reliable steady state heat removal systems that can handle high heat fluxes; c) Steady state impurity removal and control demonstration; and d) H-mode (or good confinement via Super Shots, etc.) demonstration in steady state. Ergodic magnetic limiters may help with these issues, but the confinement and impurity control capabilities with this system is unknown. 


\section{B. Technology Issues}

1. Materials. Steady state operation will make material selection even more critical than in present machines. The plasma interactive components (PIC) material selection will be determined by the edge plasma and divertor plasma parameters, which control the impurity production and transport to the core, and the requirements for disruption survival. Better materials for PIC are needed, and the investigation of composite weaves and ceramics during plasma bombardment and neutron irradiation should be pursued. The fabrication of PIC materials and their joining to the stuctural and cooling materials is an important issue. Strong differential expansion between low $\mathrm{Z}$ materials and the cooling structures during neutron irradiation make the joint reliability an issue. Corrosion and erosion of the coolant tubes is also an issue.

2. Heat Transfer and Thermal Stress. Active cooling of high heat flux (HHF) components is an important issue. The maximum power density that can be removed continuously will determine PIC designs. The thermal gradient in material exposed to a given heat flux limits the thickness of the material due to stress build up, which strongly impacts the lifetime of PIC exposed to steady state high heat fluxes. HHF test facilities (PMTF) and the actively cooled limiters under development for TORE SUPRA are staring to address these issues.

3. Neumon Irradiation. Modifications in the materials properties is definitely a critical issue, with the magnitude depending mostly on the fluence produced by ETR. Even if the bulk properties are not significantly changed, interfaces berween plasma facing materials and their support/cooling structures are very sensitive and may be a problem. Irradiation effects depend strongly on the temperature and gradients in the materials, and no data base presently exists for most of the PIC materials.

4. Erosion and Redeposition. Erosion is a major issue for impurity production and component lifetime in a steady state machine. The data base on erosion rates of low $\mathrm{Z}$ materials is good from ion beam experiments and recent laboratory plasma experiments (PISCES). However, the measured total sputtering yields are high and result in significant erosion in tokamak edge plasma conditions. The proposed control of the edge plasma temperature to reduce 
the sputtering of PIC is relatively ineffective for the low $\mathrm{Z}$ plasma facing materials candidates because of a lack of a strong energy dependence in the erosion yield. This is due to the chemical sputtering of graphite and the low threshold for beryllium. High $\mathbf{Z}$ materials offer a high threshold so that an edge temperature control scheme might significantly reduce the erosion, but tokamak experiments (except for Alcator-C) have not demonstrated low impurity levels with high Z plasma interactive materials. Better materials are desired, and work on advanced ceramics should continue.

The redeposition data base is emerging due to recent results from the PISCES experiment and observations in tokamaks. Redeposition is observed to reduce the net erosion rate of suffaces. However, the plasma tends to erode and redeposit at different rates in different locations. Regions of net erosion and net redeposition are observed, which implies that redeposition cannot yet be relied on to control the erosion of PIC unless some control of the location is achieved.

5. Tritium Inventory and Permeation. The tritium inventory problems with present machines will be mostly alleviated by a proper site selection for a steady state ETR type machine. Retention in the PIC is an environmental and safety issue. Permeation of tritium into the coolant systam is a critical issue due to the expense of removing and piocessing large amounts. Permeation barriers need to be developed for fusion conditions where heat removal and the joint reliability have an important role. The tritium data base is increasing due to results and continuing research in the TPX facility.

6. Disnuptions. Surprisingly, disruptions are a major issue for seady state machines. The performance of components for steady state heat removal, and PIC designs with adequate lifetimes, are severely compromised by requirements for disruption survival. At the minimum, the number of disruptions must be limited in the device, and hopefully disruptions can be controlled or eliminated entirely by early precursor detection and feedback control systems.

7. Heating and Fueling Components. Issues exist external to the tokamak, such as developing high power neutral beam systems, high frequency microwave sources, and active if 
tuning systems, capabie of steady state operation. Issues internal to the tokamak are identical to the issues facing PIC, with impurity generation by $r$ heating systems being tha major issue.

8. Diagnostics and control. The modification of diagnostics for steady state operation can be a significant issue in many cases. A dependence on robust optical diagnostics is indicated, and new techniques will have to be deveioped to replace the standard magnetic loops and bolometers now used in pulsed machines. Receilt advances with reflectometers and the LIDAR scattering systems are promising for steady state plasma density and temperature measurements. Developmerts are needed to increase the availability and reduce the calioration time requirements of most diagnostics.

\section{Issues in Alternative Corcepts}

\section{Reversed Field Finch (RFP) and Spteromaks}

a. Physics. Issues. As discussed in the physics summary section, non-inductive current drive is a critical issue for RFys. Understanding the physics which determines $\beta_{\theta}$ and $\tau_{\mathrm{E}}$, and the effect of the profiles on these parameters, is very important. The RFP has dynamo activity which sustains the mean field profiles in the plasma. The effect of $\mathrm{v}$.is sustainment mechanism on plasma transport, and the scaling with current and/or current density must be determined.

b. Stabilizing Conductiorshell. A conducting shell in close contact to the plasma and may be requirec for the stability of RFPs. Determination of the important features and parameters of the conducting sheli is an important issue. Active feedback controi of the plasma equilibrium is needed for steady state. The effect of introducing significant holes in the stabilizing shell, i.e. for divertors, may also be important in determining the stability of future devices.

c. Impurity Control. As discussed for tokamaks, impurity control is an important issue for RFP devices. Similar limiter/divertor flate areas are required to remove similar amounts of power; therefore most of the issues are the same as for tokamaks. The high power density in an: RFP on the first wall requires the use of a larger fraction of the first wall to remove the power, 
and needs reliable steady state cooling systems. Pump limiters for an RFP require a gap between the limiter and the wall as the location of particle collection. The possible requirement of a close conducting shell for stability may reduce the effectiveness of a pump limiter. Divertors have the same problem with this shell, holes or channeis leading to the divertor may affect stability. Divertors close to the plasma, similar to the open divertors in tokamaks, are now considered to solve this problem. The divertor plate heat flux may be significantly larger than in a tokamak.

d. Disnuptions. The problem of disnuptions will be similar for RFPs as for tokamaks.

\section{Devices and Laboratory Facilities to Address Steady State Issues.}

The problems involved in the issues summarized above nust be solved before a steady state ETR or ITER machine can be successfully operated. The confinement devices and lahoratory facilities which can address these issues are listed in Tables 1 and 2 for the time intervals of 0 to 5 years and 5 to 10 years. Several of the devices and facilities already have programs to address specific steady state issues, as shown in the tables. Many of these issues will be addressed to some degree in the next generation long pulse machines, specifically TORE SUPRA, ATF, and LHS, when they achieve significant pulse lengths. The extent to which the specific problems can be solved in these devices must be assessed in a forthcoming program plan.

The U.S. laboratory facilities can provide very comprehensive data on steady state issues, especially in the technology field. Most of the PMI and HHF facilities already have some steady state capability. These facilities can be used to address the issues identified here if they are directed away from near term issues and toward steady state issues.

From the tables it is apparent that some issues are not yet, or cannot be, adequately addressed. Disruption control and current drive, whisil are crucial for steady state, need to be emphasized in prograrns on confinement machines. The data base for neutron irradiation effects on fusion materials, component structures, and interfaces is very limited. The Ho: Electron Beam Facility (HEBF) must be completed to provide this information. In addition, materials issues and diagnostics and control system development for steady state devices must be vigorously pursued. 
Table 1. Devices and Facilities available to address Physics Issues for Steady State.

\begin{tabular}{|c|c|c|c|}
\hline $\begin{array}{l}\text { PHYSICS } \\
\text { ISSUE }\end{array}$ & 0.5 years & $5-10$ years & $10+$ years \\
\hline Current Drive & $c, E, f, i, N$ & $\mathbf{N}$ & \\
\hline Profile Control & $\mathbf{B}, \mathbf{F}, \mathbf{H}, \mathbf{i}, \mathbf{k}, \mathbf{M}, \mathbf{N}$ & $\mathrm{d}, \mathbf{N}$ & \\
\hline Disruption Control & $\mathbf{b}, \mathbf{n}$ & & ETR \\
\hline $\begin{array}{l}\text { Impurity Transport } \\
\text { and Control }\end{array}$ & $\mathbf{A}, \mathbf{B}, \mathrm{c}, \mathrm{h}, \mathbf{i}, \mathbf{L}, \mathrm{n}, \mathbf{2}$ & $d, j$ & NET \\
\hline $\begin{array}{l}\text { Particle and Impurity } \\
\text { Rernoval }\end{array}$ & $A, B, i, L, N$ & $\mathrm{~d}, \mathrm{j}, \mathbf{N}$ & ITER \\
\hline$\alpha$-Particle Physics & (h?) & D & \\
\hline
\end{tabular}

\section{Devices and Facilities}

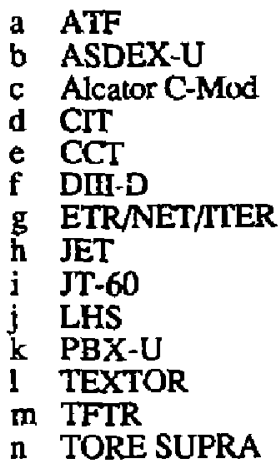

1 PMTF

2 PISCES

3 TPX

4 HEBF

5 FELIX
Date of Operation

1988

1989

1989

[planned]

1993

operating

opera:ing

2000

operating

operating

[planned]

1992

1988

operating

operating

1988

operating

operating

operating

[planned] 
Table 2. Devices and Facilities available to address Technology Issues for Steady State.

\begin{tabular}{|c|c|c|c|}
\hline $\begin{array}{l}\text { TECHNOLOGY } \\
\text { ISSUE }\end{array}$ & $0-5$ years & $5-10$ years & $10+$ years \\
\hline Materials & $1,2, L, "$ & $1,2, *$ & \\
\hline $\begin{array}{l}\text { Heat Transfer and } \\
\text { Thermal Stress }\end{array}$ & $1, a$ & 1 & \\
\hline Neutron Irradiation & 4 & 4 & ETR \\
\hline $\begin{array}{l}\text { Erosion and } \\
\text { Redeposition }\end{array}$ & $2, *$ & $2, *$ & NET \\
\hline Tritium & $3, \mathrm{~m}$ & $3, d$ & ITER \\
\hline Disruption effects & 1,6 & & \\
\hline $\begin{array}{l}\text { Heating and Fueling } \\
\text { Components }\end{array}$ & $\mathbf{N}, *$ & $\mathbf{N}^{*}$ * & \\
\hline $\begin{array}{l}\text { Steady State } \\
\text { Diagnostics and Control }\end{array}$ & $h, n, 2, *$ & $\mathrm{j}, \mathrm{n}, 2, *$ & \\
\hline
\end{tabular}

notes: - BOLD letters indicate program emphasis,

* could be addressed to some degree in existing confinement machines

- device symbol meanings given in Table 1. 


\title{
Part A
}

Technical Assessment of Critical Issues for Steady State Operation

\author{
Physics Issues for Steady State Operation
}

Contributors

\author{
A.J. Wootton', Chairman \\ R.W. Conn ${ }^{2}$ \\ P.K. Mioduszewski ${ }^{3}$
}

${ }^{1}$ Fusion Research Center, University of Texas at Austin, Austin, TX 78712

2 Institute for Plasma and Fusion Research, University of California, Los Angeles, CA 90024

${ }^{3}$ Oak Ridge National Laboratory, Fusion Energy Division, Oak Ridge, TN 37830 


\section{INTRODUCTION}

This section attempts to list the important physics questions which must be addressed if a steady state fusion device is to be operated. Conversion from pulsed to steady state condition introduces some specific issues. The most important of these are a) whether a non-inductively driver plasma current can be maintained in a tokamak, b) the long-term evolution of profiles and the resulting stability conditions (especially important are the effects of self-generated alpha particle heating) and c) the problems of particle and impurity control. In the following text the physics issues are discussed under the headings:

1) The equilibrium

2) Stability and profile control

3) Density control

4) Impurity control requirements

5) Burn control

6) Helium ash removal

7) Edge plasma conditions

8) Impurity control systems in tokamaks

For each subsection the main problems are discussed, and possible solutions presented. Those issues which are common to both steady state and pulsed devices $a-c$ generally ignored. Important experiments needed to clarify certain points are identified. The concluding section describes the impurity control techniques presently used in tokamaks and their issues for steady state operation. 


\section{The Equilibrium}

Starting at the lowest level, a steady state plasma requires a steady state equilibrium. There are three generic machine types presently considered as contenders for a magnetic fusion reactor, namely tokamaks, stellarators (and their associates, torsatrons and heliacs) and reversed field pinches. Of these, only the steilarator family provides a steady state equilibrium through extemally imposed fields. This is at the expense of complexity of the poloidal field winding configuration. Each of these generic types is now considered separately.

\section{a) The Tokamak}

The equilibrium is partly supplied by the self field of the plasma current, which decays resistively. A certain pulse length can be achieved using a transformer, before some additional current drive is required. In any electron current drive system the radio frequency power must be sufficient to maintain a population of suprathermal electrons with energy $E_{\text {res }}$ against the frictional forces arising from Coulomb collisions. The resulting current drive efficiency is given by

$$
\mathrm{I}(\mathrm{MA}) / \mathrm{P}(\mathrm{MW})=2.4 \mathrm{E}_{\mathrm{res}}(10 \mathrm{kkeV}) /\left(5+\mathrm{Zeffn}_{\mathrm{e}}\left(10^{20} \mathrm{~m}^{-3}\right) \mathrm{R}(\mathrm{m} \mathrm{s})\right)
$$

Thus, the efficiency decreases with increasing $n_{e}$ and major radius $R$ : it is imporant to maximize $E_{\text {res. }}$ Various forms of current drive are being considered both experimentally and theoretically, namely

(1) Electron cyclotron current drive.

This suffers from limiting plasma densities and requires gyrotron development to higher frequencies, although schemes using lowered frequencies are under investigation.

(2) Lower hybrid carrent drive. 
The right combination of low parallel index of refraction and high $E_{\text {res }}$ are found with lower bybrid slow waves. At high toroidal fields efficient drive has been exhibited on Alcator and PLT. The required efficiencies to achieve initial current ramp up and accommodate periodic transformer recharge have already been achieved experimentally. However, at high densities with low toroidal fields the slow waves are not predicted to penetrate, so fast waves will probably have to be used.

(3) Oscillating field current drive.

This proposed technique is as yet unproven. Its first application is expected to be to reversed field pinches. The STX tokamak experiment, proposed by ORNL, will also investigate this technique.

(4) Neutral beam current drive.

This technique, demonstrated on the DITE tokamak, is nct generally thought to extrapolate to reactors. However, neutral beams may be present in a reactor for heating or burn control, and, therefore, neutral beam current drive or beam-assisted if current drive current may be possible.

(5) Alfven wave current drive

This technique has not yet been experimentally tested. It has the advantage of driving current approximately on a flux surface, with high predicted efficiencies.

An altemative to a true steady state tokamak is one where the plasma current reverses sign periodically, or uses two alternating adjacent equilibria with opposite directed plasma clirrents. Such systems must be reheated to ignition on each cycle, but it may be possible to store thermal and electromagnetic energy at the end of each burn cycle. However, no setisfactory solutions have been presented for the controlled rampdown of current and density in the case of the sirernating current scheme, and the proposal of two adjacent equilibria involves complex magnetic designs. 
b) Stellarators, heliacs and torsatrons

These devices have an equilibrium maintained by external currents, so that current drive is unnecessary. However, internally driven currents (from finite pressure, heating systems and loss orbits) can lead to the creation of magnetic islands and subsequent loss of confinement in low shear systems. Externally driven currents may be necessary to control these internally generated currents. If this is the case, then the current drive mechanisms listed for tokamaks must be used for stellarators.

\section{c) The reversed field vinch}

This configuration is hoped to reach ignition and beyond with only Ohmic heating. Existing devices have operat id with close-fitting conducting shells to maintain the flux reversal and stability. Experiments with segmented shells arc important for vilimate steady state operation and are being pursued.

It has been observed that all reversed field pinches can last longer than model predictions using axisymmetric resistive field diffusion. Also, the associated fluctuations are above the thermal tevel. The field configuration is thought to be maintained by a jrocess called the dynamo effert. It remains to be seen if this process occurs at high temperatures (and thws high magnetic Reynolds numbers). Recent theoretical work suggests that the reversed field configuration can be maintained in a quasi steady state by modulating the poloidal and torcidal fields at audio frequencies. An important question is whether this technique works, especially as the edge temperature increases. Álso deleteriọs modifications to transport may result. 


\section{Stability and Profile Control}

In the experimental area, diagnostic enhancements have increased knowledge of linear stability. Improvements in fueling and heating technology have allowed considerable control in discharge stability. Techniques for the tailoring of plasma parameters include current and density ramping (gas puffing and pellets), localized heating (especially electron cyclotron resonance heating) and current drive. With these techniques many "best" discharges in smaller machines are achieved in conditions well away from steady state. Often neither the plasma current nor the density is constant. Results from larger machines (JET, TFTR) look hopeful; it appears that good conditions can be achieved in true steady state conditions. However, the available data base of discharge parameters should be used to identify any difference in maximum parameters (beta, mnfinement times, density, safery factor, etc.) when transient and steady state conditions are used.

Two control techniques are of special interest in tailoring profiles: localized heating and localized current drive. Spatially localized heating is expected from electron cyclotron resonance heating, ion cycictron resonance heating, Alfven wave heating, lower hybrid heating, and to a less extent, neutral beam injection. Thus, some control over both electron and jon heating profiles is available. Localized current drive is expecied from lower hybrid current drive, electron cyclotron current drive, and neutral beam current drive. Existing experiments cars identify the advantages of these control techniques in optimizing plasma parameters. The effects or -- narately controlling heating and current profiles on turbulence should be studied.

Certain optimized configurations are being investigated for allowing stable high beta plasmas. These include producing high beta in the first stability regime (e.g. the proposed tokamak STX at ORNL) and producing high beta by accessing the seccnd stability regime (e.g. the PPPL tokamak PBX, and the ORNL torsatron ATF). It has also been proposed to obtain access to the second stability regime by using large R/a tokamaks, although a narrow unstable 
region is still predicted which must be crossed (e.g, the SRX iokamak). All these devices may require profile control to obtain the hoped for profiles.

The concept of profile consistency is found useful in moderate beta tokamaks; this argues against the possibility of profile control. It remains to be shown whether this is a useful concept at high betz. 


\section{Density Control}

Particle control in long pulse or steady state fusion devices is a necessary prerequisite for particle balance and a potential means for improvement of plasma performance. Density and inupurity control are always linked. A control system for either acts by modifying sources, fluxes, sinks, or any combination of three. In pulsed systems it is simple to modify the sink by changing the recycling coefficient $R$, e.g. by gettering. At high wall recycling $(R \approx 1)$ and steady state operation, externa' particle sources such as gas puff, pellet injection, or neutral beam injection have to be balanced by corresponding exhaust. In addition to the working gas, Helium ash has to be removed in D-T burning devices in order to hold the ash concentration at a given level (e.g. $\leq 5 \%$ ). Comparison of pellet and gas fueling on the one hand, and recycling control with divertors, limiters, or gettering on the other hand, indicates that the control of particle sources and sinks is a key element for the optimization of plasma performance. While the continuous exhaust of plasma particles with divertors or pump limiters is established, the control of sources, sinks, and recycling for optimum plasma performance is far from being understood, although it has shown some success in the past.

\section{a) Sources}

The sources used for steady state would be the same as for pulsed operation, namely, gas puffing or pellet injection (increased velocities to $15 \mathrm{~km} \mathrm{~s}^{-1}$ are required). Neutral beams can also be used, but are an energetically expensive method. However, they may already be present as an auxiliary heating system, e.g. for burn control. Other techniques less well investigated are Marshal gun plasma injection and cluster injection.

The most obvious correlation between fueling method and plasma performance has been observed with pellet fueling compared to gas fueling. Pellet fucling has the potential of changing the plasma source distribution in a way that alters the plasma profiles and improves plasma performance in terms of confinement, impurity concentration, and density limits. While on most 
devices pellet fueling is being used to inprove performance, systematic studies of various aspects of pellet fueling, like e.g. pellet penetration and profile build-up, are no: available. For steady state devices, a crucial question is whether of not it will be possible to maintain favorable profiles with pellet fueling for a period of time longer than the central particle confinement times. After times comparable to several central particle confinement times, particles have diffused out of the core and built up recycling in the edge. It seems to be obvious that edge recycling needs to be kept sufficiently low in order to prevent filling in the peaked profiles from the plasma edge.

If this is a prerequisite for maintaining the desired profiles, only divertors with the capability of exhaust efficiencies near $100 \%$ would be adequate. It is not clear that $100 \%$ exhaust efficiency in continuous operation is a relevant scenario technically due to the very large gas load and high pumping reguirements. Devices with pump limiters and exhaust efficiencies around $10 \%$ of the plasma efflux, and are likely to be recycling-dominated at continuous operation. In fact, efficient pump limiter requires high recycling at the edge. It is not obvious at present if an operating scenario with peaked profiles, maintained by pellet fueling, and high recycling localized in the edge for efficient pump limiter operation is possible.

Central particle confinement times of several seconds have been observed in today's largest devices. In Tore Supra, pellet fueling for up to $30 \mathrm{sec}$ with particle exhaust by pump limiters is foreseen. This should be adequate to study the prospects of continuous pellet fueling with a pump limiter exhaust scheme. For a corresponding scheme in a divertor machine, ASDEX Upgrade seems to be most suitable. It is designed for pulse lengths of up to $10 \mathrm{sec}$, and has some pumping capability in the divertor chamber.

In addition to systematic studies of pellet fueling, the benefits of other fueling schemes should be investigated, such as gas fueling through the limiter head to avoid ionization in the SOL, or plasma fueling through divertor or pump limiter throat. 


\section{b) Recycling}

Recycling is the dominate fueling source in most configurations. In applications with sufficient pulse length and no external particle control scheme, recycling becomes the only fucling source. In long pulse devices in which the wall interactions are dominated by metallic (stainless steel) walls, discharges are maintained without any external source of fueling. Models for recycling on stainless steel include reflection, retention, diffusion, and recombination have described previous experimental results reasonably well.

Recycling on carbonized walls or graphite, on the other hand, is very complex and a coherent model suitable to be used in transport codes is not available yet.

Recent results on TFTR ('Supershots') have demonstrated that graphite can be conditioned for low recycling, leading to improved plasma performance. But, so far, this is a transient phenomenon and it is unlikely that this effect will be relevant at continuous operation.

\section{c) Sinks}

In short pulse devices $(t=1 \mathrm{sec})$ some particle exhaust is automatically provided because of the finite pumping capacity of the walls. Depending on history and preparation of the walls, recycling coefficients between 0.5 and 0.9 are typical for short pulse operation. With increasing pulse lengths, the walls saturate and lose their ability to pump. Two altematives exist: pump limiters and divertors.

\section{1) $\underline{\text { Limiters }}$}

Continuous particle exhaust can be provided with magnetic Jivertors or with pump limiters. In a pump limiter the sink is provided by pumping within an apertured limiter head. The principle concem is that the pump limiter now defines the plasma edge. The protection of this leading edge is all important. Various schemes are discussed to avoid this leading edge problem. If the density and heat flux exponentiating lengths are known, a particular limiter geometry can be used to minimize the problem. Changing exponentiating lengths can 
be tolerated by changing plasma position. However, this is an unlikely solution because plasma position control is important in obtaining optimum plasma parameters.

The biggest problem associated with steady state is heat removal. Two schemes have been suggested to increase the paricle collection efficiency and simultaneously divert heat away from the leading edge. The first, called the intra island pump limiter or magnetic islard divertor, uses magnetic islands produced by external helical coils. The second uses $c 1,1 \mathrm{~s}$ within the limiter head to modify the local toroidal field (similar to a bundle divertor) thus also diverting the particle and heat fluxes. It is important to understand experimentally if any of the pump linuter schemes can produce the so-called high recycling regimes. High recycling is found important in poloidal divertor operation for attaining the high energy confinement regimes ( $H$ modes). In reversed field pinches, it is not clear if a pump limiter is compatible with the requirements of a close-fitting shell.

Pump limiters are suitable to exhaust between 5 and $10 \%$ of the total recycling particle flux. This is sufficient to balance particle input by neutral beam injection and some modest additional fueling. However, it requires high recycling in the edge, and it is presently unclear if this is compatible with good confinement ( $r$-modes) and peaked density profiles ( $P$ modes). Recent results from JFT-2M indicate that $\mathrm{H}$-modes are achievable during operation with a pump limiter. Experiments in Tore Supra will examine the ability to maintain peaked profiles from pellet injection during long pulse operation with exhaust by pump limiters.

\section{2) Divertors}

A more complicated sink is provided by magnetic divertors. These also assist in impurity control. Four main divertor types are available: the bundle divertor, the poloidal divertor, the toroidal divertor and the helical divertor. All of these have demonstrated good particle exhaust, especially the toroidal and poloidal systems. The same two systems have demonstrated the best impurity control. In the case of the poloidal divertor high energy confinement $(\mathrm{H}$ mode) can be achieved under conditions of high local recycling at the divertor 
plate. An important point to note is that the attainment of this $\mathrm{H}$ mode is associated with higher impurity confinement, and impurity accumulation on axis. If magnetic divertors are to be used for working gas control, and the $\mathrm{H}$ mode is necessary for reactor operation, an alternate means of controlling the impurity accumulation (such as asymmeric working gas flow) must be found. It is not clear if a divertor can be fitted to a reversed field pinch configuration.

Divertors have the capability of a high exhaust efficiency, and the resulting low recycling fluxes and low neutral densities in the main plasma chamber appear to be favorable for good confinement. Although divertor machires have not been operated in: the continuous exhaust mode in the past, because of other priorities, present experimental evidence indicates that high particle exhaust and low recycling in the main chamber can be achieved with divenors in continuous operation. Experiments in machines such as JT-60 and ASDEX Upgrade will have to determine the required exhaust rates during long pulse operation to produce $\mathrm{H}$ modes, peaked profiles during pellet injection, and good plasma performance.

The design of both pump limiters and divertors for working gas (i.e. hydrogen isotope) control is improving. Three dimensional calculations of neutral effects have been coupled to particle and heat flow both along and atross field lines. However, in all cases the basic edge plasma transport must be assumed. This is discussed further in section 7, edge plasma conditions. We note that predicted performance of the modular pump limiter systems for large machines (e.g. Tore Supra) is marginal, although low recycling toroidal belts (TFTR, JET), and toroidal belt pump limiters (TEXTOR) have shown good performance. 


\section{Impurity Control Requirements}

The maximum permitted values of both central and edge impurity densities $n_{z}$ are $n_{z} / n_{i} \equiv 2 \%$ for low $\mathrm{Z}$ impurities and $\cong 0.1 \%$ for high $\mathrm{Z}$ impurities. For central values above this a fusion burn will be quenched by radiative losses. For edge values above this a thermal collapse is predicted. Thus impurity production and accumulation must be minimized. A satisfactory situation for a pulsed discharge is not necessarily satisfactory for steady state operation. A simple model based on the continuity equation for the impurity density $\mathrm{n}_{\mathbf{z}}$ produced by a impurity flux $\Gamma_{2}$ during a pulse length $\Delta t$ gives

$$
n_{z}=\Gamma_{z} \tau_{z} S\left[1-\exp \left(-\Delta t / \tau_{z}\right)\right] / W
$$

with $\tau_{\mathbf{Z}}$ the impurity confinement time, $S$ the surface area and $V$ the plasma volume. Thus, in a pulsed device $w$ might have $\Delta t / \tau_{z}$ small so $n_{z} \cong 0$. Extending the pulse duration to approach steady state gives $n_{z}=\Gamma_{z} \tau_{z} S$. Without krowing $\tau_{z}$ we can only ensure operation by reducing $\Gamma_{\mathbf{z}}$; i.e., we must reduce the edge source as much as possible.

Impurity control techniques can be categorized under the three headings of source, sink and flux control. Each of these is accomplished in a variety of ways. Sources are modified by material selection, conditioning and edge conditions. Sinks arc provided by getrering, pump limiters and divertors. Flux control is provided by magnetic forces (i.e. divertors), electric field forces, momentum input forces, and frictional forces.

\section{a) Diventors issues}

A device which provides all thr ze (source, sink and flux control) is the divertor. At present, the divertor is considered the best candidate for controlling imptrities. There are four types of divertors which have been experimentally tested: the poloidal, toroidal, bundle and helical divertors. The two systems with the exhaust and screening efficiencies combining to give the largest impurity reduction are the poloidal and toroidal divertors. Most results 
have been obtained with poloidal divertors. However, poloidal divertors have not yet been made to operate with the $\mathrm{H}$ mode and good impurity control. Experiments on ASDEX show that a stable edge plasma with $\mathrm{H}$ mode confinement lead to impurity accumulation and a consequent thermal collapse. It is not clear if any divertor system can be applied to the reversed field pinch configuration. The stellarator family of machines has an inherent divertor geometry, but no experiments have utilized this so far.

b) Edge temperature control

The plasma edge conditions determine impurity influxes, and the design of limiters, divertors and launching structures, which themselves provide the source of many of the impurities. Both sputtering yields from ions and charge exchange neutrals, and desorption yields, increase with increasing plasma edge temperature. Thus, any way to reduce the edge temperature will reduce edge impurity fluxes and thus central concentrations.

Methods for providing a low edge temperature include the radiating plasma mantle (proposer for JET), the expanded boundary, the ergodic magnetic limiter, and the high recycling regime. The latter three require high edge densities to remove the power.

c) Flux modification

A simple technique to reduce central impurity densities is to provide a locally outward impurity flux at the plasma edge. This has been shown to be possible using many of the forces discussed in section 4a. Two which are particularly simple and should be further studied are the outward forces produced by local electric fields, and the forces produced by localized magnetic islands. 


\section{v. Burn Control}

The thermonuclear heat source will play a large role in determining the detailed profiles of temperature and density, factors which will influence stability and transport. To operate at a steady stale economically means operating at maximum beta. Thus, it is important to know what happens as the burn drives pressure driven MHD modes. Is the transport slowly increased to ensure marginal stability, or is there a catastrophe such as a disruption? In particular, the usually proposed operating point of $10 \mathrm{keV}$ in a D T plasma is unstable to thermal excursions, which will have to be controlled unless the marginal stability scenario is found.

The burn controls available are

1) Control of density (see fueling section).

2) Control of temperature by impurity injection or vertical field control.

3) Controlling the D-T mix.

4) Maintaining a low ignition margin and controlling plasma with an auxiliary power source.

5) Ripple loss of alpha particles.

In stellarator type systems, the necessary burn control may occur naturally because the temperature may be limited by the collision frequency dependence of the ion confinement, and the (1/collision frequency) dependence of the electron confinement, and their coupling through the ambipolar electric fieid. 


\section{Helium Ash Removal}

An internal source of impurities is the He "ash" which results from the D-T reactions. This radiates through bremsstrahlung, contributes to fuel dilution, and may also contribute to plasma pressure driven instabilities. In steady state this ash must be removed by a sink at the edge. Estimates show that $n_{\mathrm{HE}} / \mathrm{n}_{\mathrm{i}}<5 \%$, or the fusion reaction rate will be reduced too much and the fusion bum will be quenched in tens of seconds. The issue is whether an exhaust system (pump limiter or divertor) can be built which will remove the ash before the burn is quenched.

We can relate the exhaust efficiency $\xi_{x}$ required of any impurity control scheme to the particle confinement time $\tau_{\mathrm{He}} \cdot \xi_{\mathrm{x}}$ is the fraction of the flux crossing the plasma boundary which enters the control system. In steady state the He (or alpha) particle flux produced by the fustion reactions, $\Gamma_{\alpha}$, must equal that removed by the exhaust. Then

$$
\begin{aligned}
& \xi_{x} v_{n_{H e}} / \tau_{H e}=C n_{j} 2<\sigma v>V / 4=\Gamma_{\alpha} \\
& \text { i.e., } \quad \xi_{x}=C n_{i}<\sigma v>\tau_{H e} / 4 .\left(n_{H e} / n_{j}\right)^{-1}
\end{aligned}
$$

Here $V$ is the plasma volume, $C=2$ accounts for profiles, and average densities are implied. We obtain

$$
\xi_{\mathrm{x}} \cong 6 \times 10^{-3} \tau_{\mathrm{He}}\left(\mathrm{n}_{\mathrm{He}} / \mathrm{n}_{\mathrm{i}}\right)^{-1}
$$

Two limiting cases are considered. The first places an upper limit on the allowed alpha particle confinement time. We know we must have $n_{\mathrm{He}} / \mathrm{n}_{\mathrm{i}}<5 \%$ to ensure a sufficient burn. If the plasma physics dictates $\tau_{\mathrm{He}}>7$ s even a perfect cortrol system $\left(\xi_{\mathrm{x}}=1\right)$ will not prevent a quench. 
The second limiting case estimates the exhaust efficiency needed if the alpha particle are to slow down and heat the plasma. If this time is classical, the $\tau_{\mathrm{He}}>1$ and $\xi_{\mathrm{X}}>0.12$. This is the minimum $\xi_{\mathrm{x}}$ that must be obtained.

High edge densities $\left(>10^{14} \mathrm{~cm}^{-3}\right)$ are necessary to achieve high throughput for adequate He removal. This could be achieved by recirculating some of the exhaust gas in the scrape-off layer, thus enhancing the divertor or pump limiter flow.

The combination of preventing density buildup and impurity accumulation, ensuring sufficiently high energy confinement times, and allowing the alpha particles to heat the plasma but not dilute it, present an interesting challenge to the fusion community. 


\section{Edge Plasma Conditions}

Edge plasma conditions are an important part of any reactor design considerations because they determine both impurity generation and limiter or divertor design. Because steady state operation is more susceptible to impurities than pulse operation, and because density and heat removal are more important, edge conditions become especially important for steady state devices.

Plasma outflow (of heat, momentum and particles) through diffusion and convection couples the main plasma to the limiter or divertor. The plasma edge conditions are then determined by the limiter and divertor geomerry, the neutral particle history, and not least the plasma transport coefficients. Three-dimensional calculations of neutral particle trajectories have been coupled to models of flow along and across field lines. However, most limiter and divertor designs assume an exponentiating length at the plasma edge and work with this. Enough data exists to be sure of the scalings of both density and temperature exponentiating lengths with both global and local parameters. Such a data base should be constructed.

In fact, the correct method is to work with a set of transport coefficients. Again, existing data could be analyzed to provide the scalings of these coefficients in terms of local parameters.

Experimentally it is known that both poloidal and toroidal asymmetries exist. Some of :hese are representations of shadows of material surfaces projected down field lines. However, the observation of larger densities and exponentiating lengths at the outer equator cannot be explained and should be further investigated. Poloidal asymmetries may influence the placings of either divertors or limiters.

It is important to be able to control the edge parameters. As discussed in section $4 \mathrm{~d}$, this can be comparatively simply done, using either electric or magnetic field perturbations. Once a reactor is designed, such simple systems will be the only available means of control. Existing experiments should be encouraged to further explore techniques for edge parameter control. Most machines already have suitable diagnostics. 


\section{Impurity Control Systems in Tokamaks}

Impurity control in tokamak machines refers to the need to limit the impurity content in the plasma, thereby limiting both the radiation losses from the plasma and the reduction in allowable fuel density in a finite beta system. Impurity control refers technologically to the control of the location and interaction intensity of the heated plasma with the in-vessel components. Two primary approaches now used in tokamaks are referred to as limiter and magnetic divertors. In this section we describe the concepts and operation of these systems, generally identifying the niajor critical issues anticipated for steady state operation.

\section{1) Limiters and Pump limiters}

In essentially all operating tokamaks, a special component referred to as a limiter is used to isolate the plasma from the vacuum vessel. The plasma minor radius is defined as that radial point where the plasma first contacts the material limiter. Plasma extending beyond that point is called the scrape-off layer (SOL). The plasma temperature and density at this first point of contact ranges from 50 to $500 \mathrm{eV}$ and $10^{11}$ to $10^{13} \mathrm{~cm}^{-3}$, respectively. In the SOL plasma, the density generally decays radially with an e-folding lengin of 1 to $5 \mathrm{~cm}$, depending on the machine design, limiter design, and magnetic field strergth. The density is generaily several orders of magnitude lower at the vacuum vessel. The limiter itself is designed to have an approximately contoured shape, both poloidally and torcidally, to minimize the maximum heat flux. Historically, limiters were designed using refractory metals such as Mo or W because of their high melting points, high thermal capacity, and, in the case of tungsten, the relatively high threshold energy for sputtering by hydrogen. Nonetheless, it was found in all machines, and especially the more powerful devices beginning with PLT, that sputtering of limiter material by scrape-off layer plasma bombardment caused excessive impurity contamination and radiation in the core plasma. As a result, all modern tokamaks, including the only powerful tokamak 
designed for 30 second pulses (TORE SUPRA) use graphite as the limiter material facing the plasma. Graphite has particularly good high temperature properties and low atomic number.

For steady state operation, limiters must be designed to simultaneously remove heat and exhaust plasma. Limiters which exhaust plasma are referred to as pump limiters, and they permit density control and, in reactors, helium removal. Generally accepted estimates for the required exhaust removal percentage is $10 \%$, i.e., it is desirable to remove $10 \%$ of the plasma efflux diffusing into the scrape-off layer from the plasma core.

From a design viewpoint, this exhaust is accomplished by having the edge of the limiter extend several (usually about 2) e-folding lengths into the scrape-off layer. Plasma beyond this leading adge is allowed to flow behind the limiter face (from either side) in a channel that terminates at a so-called neutralizer plate. The neutral gas resultirg from this process is them pumped via an exhaust ducting system.

Experiments have demonstrated clearly that pump limiters can be designed to efficiently exhaust plasma. The key issues are:

1. To demonstrate adequate plasma confinement using a limiter system.

2. To demonstrate steady-state heat removal with a reliable system.

3. To demonstrate adequate control over impurity introduction into the plasma core.

Plasma confinement in systems with limiters has been intensely studied in the fusion program. Both TFTR and JET, the largest machines in the U.S. and E.C. programs, are designed to operate with limiters. Both have large-area carbon-tiled limiters forming a toroidal belt and covering the inner vessel wall of the torus. Supershots with this limiter system and improved confinement were obtained in TFTR. Recently, a similar experiment in a somewhat sinaller tokamak, JFT-2M in Japan, achieved "high mode" or $\mathrm{H}$-mode confinement while operating off both an inner toroidal belt limiter and a vertical modular pump limiter. In general, however, tokamaks using auxiliary heating and limiter operation have found the energy confmement to decrease with the input power as $\mathrm{P}-1 / 2$ in a scaling referred to as "low-mode" or L-mode 
confinement. A major issue is to achieve improved confinement while operating with limiters. The $\mathrm{H}$-mode confinement time is usually a factor of 2 higher than the L-mode value, other parameters being about the same.

Steady-state operation with limiters brings with it additional major issues. The average heat flux must be held as low as feasible. This is achieved using large area limiters such as a toroidal belt. Indeed, data regarding both the heat flux distribution and particle removal rate with a full toroidal belt pump limiter will be obtained, e.q., with the ALT-II toroidal belt pump limiter experiment in the TEXTOR tokamak. However, ALT-II and TEXTOR are not steady-state systems. Such information will only be obtained beginning with modular limiters experiments in TORE SUPRA. Through these two experiments, a data-base will be generated on the heat flux distribution over a large area (toroidal belt) limiter and on steady-state heat removal systems in tokamaks. Erosion of leading edges and the heat removal at plasma neutralizer plates will be studied. Tests and techniques are re juired in areas such as the attachment of graphite to substructures of copper and other materials.

The other key steady-state limiter issue is impurity control. As noted, impurities produced at the front face of a limiter are ionized in the edge of the core plasma. Over time periods long compared with the particle confinement time, the major question is whether any such impurities migrate to and build up in the core or whether the impurities remain isolated at the plasma boundary, recycling and redepositing at the limiter. If redeposition is strong, the net erosion rate may be reduced. General experience with present tokamaks using limiters is that impurity levels are low, and impurity-induced radiation losses are not large or dominate. The major issue will be to develop reliable high heat flux pump limiters operating steady-state and to establish that these limiters will conirol the plasma density level, adequately remove helium and heat, and not lead to a steady increase in the plasma impurity levels. Further, even if slow impurity accumulation cccurs, one must determine the characteristic buildup time and whether, by some operating procedure, such impurities could be periodically "flushed" from the system. 


\section{2) Magnetic Divertors}

Magnetic divertors are an altemative to limiters and pump limiters. In tokamaks, the predominate divertor design is to use external vertical field coils to produce one or nore magnetic nulls in the poloidal magnetic field. The nulls are located on a flux surface within the vacuum vessel. The magnetic surface containing the null is called a separatrix. Field lines on magnetic surfaces outside the separatrix surface can be magnetically diverted to a region called the divertor chamber, often away from the plasma core, where the SOL plasma is guided to interact with divertor neutralizer plates. Divertors have been shown to work and they place the zone of primary plasma-wall interaction at a relatively remote location. However, divertor plasmas are not in generai significantly cleaner of low $\mathbf{Z}$ impurities than plasmas operation with limiters.

What has been established is that tokamaks operating with a poloidal divertor achieve superior confinement time, i.e., the so-called $\mathrm{H}$-mode in which $\tau_{E}$ is roughly twice as high as it is in the L-mode regime. Furthermore, the power in the SOL plasma upstream of the divertor target can be sufficiently high as to permit density and temperature gradients along field lines approaching the target plate. Specifically, the density increases sharply (by 1-2 orders of magnitude) neat the plate while the plasma temperature drops to a low as 3 to $5 \mathrm{eV}$. This has several important potential consequences. For one, the low temperature of plasma at the plates should minimize plate erosion by processes such as sputtering. Secondly, density control should follow simply by actively pumping in the divertor zone. Thirdly, impurities which are produced from the divertor plate may be entrained in the inflowing SOL plasma and be returned to the target plate. This could prevent impurities from entering the plasma core.

Nevertheless, there are major issues for the use of magnetic divertors in steady-state devices. One issue is impurity accumulation during H-mode confinement. Do impurities enter the plasma and migrate to the core, where their confinement time may be very long? There is evidence of such impurity accumulation from existing experiments. Also, the helium exhaust rate must be sufficient $(-10 \%)$ and steady-state heat removal is a problem similar in difficulty to that with limiters. Mechanically, actively cooled structures are needed to remove heat fluxes which may 
peak at values as high as $1 \mathrm{~kW} / \mathrm{cm}^{2}$. Design flexibility may be greater with divertors because the plates do not directly face the core plasma. This could permit the of high $\mathrm{Z}$, low sputtering materials such as tungsten attached to a heat removal substructure. On the other hand, recent design trends such a the diveror for the Compact Ignition Tokamak (CIT) do not use narrow plasma flow channels (such as in early experiments like ASDEX and PDX) to block the backward flow of gas toward the core. Rather, newer designs rely on plasma buildup in front of the divertor target plates to prevent gas from reaching the plasma core. Because it has been established that $\mathrm{H}$-mode confinement can be achieved using a poloidal divertor, the divertor is being included in the base designs for two major planned devices, the CIT and the ITER (Internaticnal Thermonuclear Engineering Reactor).

\section{3) Ergodized Plasma Edge for Impurity Control}

While limiters, pump limiters, and divertors are the dominate concepts used in tokamak machine designs today, other concepts are developing and may find wider use as approaches to impurity control in the funure. One concept is the ergodic diveror, and it has received considerable attention. The idea is to create a zone near the edge plasma where transport is significantly enhanced by using small amplitude resonate magnetic perturbations along flux lines. Magnetic islands are produced which overlap, ergodizing the lines. In this way, the energy can be transported across the nominal field through a zone of constant pressure. The density should increase radially towards the wall while the temperature would decrease. Wall erosion can be minimized by the low temperanure edge, and impurities reduced by causing the plasma to be collected in, e.g., modular pump limiters selectively located around the device. However, exactly where to locate such limiters is a key issue. Impurities could be kept from the core plasma if they are entrained in the plasma flow from the core. Relatively few experiments have been done, though initial results, e.g., in the TEXT tokamak, were encouraging. Steady-state tests of this concept are planned for the TORE SUPRA tokamak with 30 second pulses. Major technical issues are generally similar to those for limiters or poloidal divertors, although special 
issues will be revealed once more definitive experiments are completed. Three clear issues are whether the ergodized boundary reduces global energy confinement time, whether F-mode confinement is possible, and whether the technical complexity of introducing ergodization coils in the next generation machines is overly complicated and costly. 


\title{
Part B
}

\section{Technical Assessment of Critical Issues for Steady State Operation}

\section{Technology Issues for Steady Stace Operation}

\section{Contributors}

\author{
L.A. Berry ${ }^{1}$ \\ P.H. Edmonds 2 \\ W.B. Gauster ${ }^{3}$ \\ D.M. Goebel ${ }^{4}$ \\ H.L. Heinisch 5 \\ D.K. Owens 6 \\ J.B. Whitley ${ }^{3}$ \\ K.L. Wilson?
}

1 Oak Ridge National Laboratory, Fusion Energy Division, Oak Ridge, TN 37830

2 Fusion Research Center, University of Texas at Austin, Austin, TX 78712

3 Sandia National Laboratories, Albuquerque, NM 87185

${ }^{4}$ Institute for Plasma and Fusion Research, University of Califomia, Los Angeles, CA 90024

5 Battelle, Pacific Northwest Laboratories, Richland, WA 99352

6 Princeton Plasms Physics Laboratory, Princeton University, Princeton, NJ 08544

7 Sindia National Laboratories, Livermore, CA 94550 


\section{Introduction}

Technology issues for steady-state operation can be found in most of the components in a fusion confinement machine. High particle flux, high heat flux, and neutron irradiation of the plasma interactive components result in difficult problems in the areas of impurity control, heat removal, materials performance and tritium permeation in steady-state machines. The often conflicting desires of achieving continuous high heat removal while being able to withstand plasma disruptions and controlling impurity production makes materials selection difficult in tokamaks, for example. The design and operation of plasma interactive components and diagnostics requires solutions to many different technology issues for steady-state operation of fusion machines.

In this part of the assessment, steady-state issues in the technology area that apply to an ETR type tokamak device are discussed. The steady-state issues emphasize plasma-materials interaction, high heat flux, and plasma-related aress, and include:

1) Issues in plasnra facing components

2) Materials

3) Thermal stress and fatigue

4) Heat transfer

5) Neturon irradiation effects

6) Erosic: and redeposition

7) Tritium issues

8) Transient behavior

9) Heating and fueling systems

10) Diagnostics and plasma control

11) Maintenance 


\section{Plasma Facing Components}

Since steady-state operation is a desirable goal for a commercially viable fusion reactor, the design and operation of a long-pulse or steady-state engineering test facility is an important step in fusion power development. A steady-state, current-driven device such as TIBER II, or any long-pulse ITER device, places severe demands on the first wall and divertor designs in terms of thermal management. On the other hand, steady-state or long pulse operation should, by reduced cycling, teduce failure rates and extend the lifetimes of many components. The following assessment of plasma facing components is based on an analysis of the nuclear performance of an ITER/TIBER machine (report to be published, C.C. Baker, editor).

Research and development for plasma interactive components for ITER/TIBER will concentrate on predicting and evaluating the performance of plasma facing materials such as the first wall armor tiles and the divertor tiles. Two major areas to be addressed are plasma operational issues and materials rescarch and development issues. Since many of the key issues are not directly tied to the D-T neutron environment, work on existing tokamaks and at other research facilities can serve to resolve many of the open questions before TIBER final design, if efforts are properly focussed. Some data on neutron related topics san also be collected by use of existing fission reactor farilities.

\section{Plasma Operational R\&D Issues}

The present TIBER design includes high- $Z$ tungsten divertor tiles with low- $Z$ tiles oî carbon (carbon-carbon composite or graphite) or teryllium serving as possible alternatives. Impurity transport and impurity accumulation are key issues in the choice between high-Z and $10 w-Z$ divertor materials. If a high recycling, low edge temperature (about $10 \mathrm{eV}$ ) divertor regime cannot be established, erosion and redaposition of high- $\mathcal{Z}$ divertor materials to the first wall will occur. Sputtering of the redeposited high-Z materials by charge exchange neutrals will lead to unacceptable levels of high-Z impurities in the plasma. Even a small fraction of a per cent of 
tungsten, for example, in the plasma leads to a significant degradation in the nuclear yield. Before high-Z divertor plates can be given serious consideration for a CIT or ITER design, experiments with a high-Z divertor must be done in ASDEX, PBX-U, or DIII-D. The current experiments on ASDEX with copper divertor plates could already give some indications of effects to be expected.

A second plasma operational issue is plasma disruption control. Suppression of disruptions will be necessary for high- $Z$ divertors in order to prevent vaporized divertor tile material from reaching the first wall. Suppression of disruptions would also permit first wall and divertor designs which are be ter optimized for the anticipated neutron fluence. Plasma current profile modification with the potential to suppress disruptions by the use of techniques such as if current drive and special control coils are being investigated in a variety of existing machines. JET, for example, is planning active disnuption control with the use of external saddle coils, and this type of effort should be encouraged.

The degree of isolation required between the plasma and the divertor chamber in order achieve long confinement time ( $\mathrm{H}$-mode) plasmas is another area where further investigations are necessary. While this is primarily a performance issue, a closed divertor chamber could also restrict movement of high- $Z$ impurities, and enhance the feasibility of the use of high-Z divertor tiles. JET, DIII-D, and PBX-U are suited for studies of the effect of varying the distance of the wall/divertor plate to the separatrix and of the effect of baffling the entrance region to the divertor. More data of this type will increase confidence in divertor designs.

Present research on tokamaks has emphasized the surdy of core plasma phenomena, with a much lower level of effort directed at understanding edge physics phenomena. A more detailed mapping of edge parameters will be of high value for the design of impurity control systems. This work can be expanded on all machines. 


\section{Materials R\&D Issues}

For plasma interactive components, primary materials development issues are related to the areas of fabrication, erosion and redeposition, neutron damage, and tritium inventory and permeation. Again, existing facilities can be used to obtain much of the information necessary for the ITER final design phase.

The fabrication and use of materials in the high heat flux, $14 \mathrm{MeV}$ neutron environment characteristic of ITER requires further investigation. For example, in the present TLBER design with tungsten divertor tiles brazed to a copper substrate, thermal stresses resulting from either thermal expansion mismatch during the braze cycle or from operational temperature gradients could result in early failure of the braze joint. If, during neutron irradiation, one of the joined materials swells more than the other, resulting stresses can also lead to failures of the joint. To minimize the thermal stress portion of the problem, the use of techniques such as inserting a soft interlayer or a sponge metal between the materials during brazing is under investigation. Minimization of problems associated with differential swelling associated with neutron damage will require a good understanding of the swelling characteristics of the materials. The construction and testing of proposed ITER/TIBER designs in existing high-heat-flux and fission reactor facilities will lead to much greater confidence in the final divertor and first wall designs.

Redeposition of eroded materials is necessary for low- $\mathrm{Z}$ materials such as carbon or beryllium to provide reasonable operating lifetimes, and further work is needed to study the redeposition process. Since laboratory experiments with monoenergetic ion beams often give different erosion rates than observed in tokamaks, and since reionization and redeposition are dependent on plasma edge conditions, redeposition studies are best done in plasma devices and operating tokamaks. Erosion and redeposition are being investigated in the PISCES facility at UCLA. Existing tokamaks, such as TRTR can be used for the study of the reder osition of graphite.

The proposed first wall material for TIBER is a carbon-carbon composite graphite. A major question with the use of carbon-carbon composites in TIBER is the susceptibility carbon 
fibers have shown to neutron damage. Tests in fission reactors 1,2 with carbon cloth and fibers have shown that general degradation of material properties occurred much faster in the cloth and fibers tested than in nuclear grade graphites. Extrapolation of these results suggests that carboncarbon composites will also have poor neutron radiation resistance. At present research ${ }^{3}$ is underway to detemine if radiation resistant carbon-carbon composites can be made.

Although tritium inventory in the wall and divertor is probably not a serious issue because of the high total on-site tritium inventory, the question of tritium permeation needs attention. In particular, the trapping sites produced by neutron irradiation determine the time in which steadystate permeation rates are reached. Studies conducted in the Tritium Plasma Experiment (TPX) and the Fast Flux Test Reactor (FFTF) can be used to obtain at least a first level of understanding of the relevant effects.

The use of beryllium as a potential plasma facing material also requires further attention. Beryllium armor tiles have the additional advantage, besides being a low- $Z$ material, of acting as an oxygen getter. This would lead to improved plasma performance through lower oxygen levels

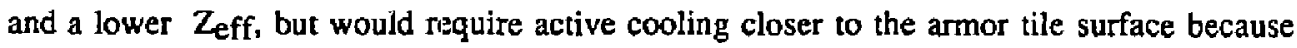
beryllium has a lower melting point, and the high temperatures (about $1500^{\circ} \mathrm{C}$ ) planned for graphite armor would not be achievable. In a fusion reactor the usual concem for beryllium toxicity may be less of a problem than in present machines since neutron activation will require that the special handling procedures normally associated only with beryllium be adopted for any first wall material. One potential problem with beryllium in fusion applications is swelling due to n-alpha reactions. Further research in fission reactors is in order to determine the extent of this problem. The Joint European Torus (JET) has beryllium wall armor and limiters available and will decide soon whether to install beryllium in JET. Results from the JET experiment will give general information on the relative merits of the use of beryllium and could significantly affect wall material decisions for the ITER/TIBER design and other fusion designs, and such work should be encouraged. 


\section{References}

1. W.J. Gray, "Neutron Irradiation Effects on Carbon and Graphite Cloth and Fibers," Report BNWL-2390, Battelle Northwest Laboratory, Hanford, Washington, August 1977.

2. R.J. Price, G.R. Hopkins, and G.B. Engle, "Effects of Fast Neutron Irradiation on Carbon Fibers," in Extended Abstracts and Program of the 178th Biennial Conference on Carbon, University of Kentucky, Lexington, June 16-21, 1985, p. 348.

3. G.B. Engle, NAMCO, Inc., San Diego, "Neutron Irradiation Stable Carbon-Carbon Composites," Contract DE-ACO3-86ER80418, May 15, 1987-May 15, 1989. 


\section{Materials Issues for Steady State Devices}

The extension of plasma discharges toward steady state operation will telieve some materials problems such as thermal stress fatigue while exacerbating other problems such as thermal creep. In general, however, the problems to be dealt with will be the same as problems for high heat flux components in current devices. For example, the list of desirable materials properties given in Table 1 applies to both pulsed and steady state devices, but the substantially increased duty times of steady state devices will further emphasize the importance of the materials selected. (For further discussion, see References 1-8).

The materials requirements for plasma facing materials are somewhat different than those for the structural materials. in general, all materials from the blanket inward will be exposed to a high energy, high flux neutron environment which will degrade the material by both displacement damage and by the formation of transmutation products, especially helium and bydrogen. Neutron effects observed in most materials include density changes (usually swelling) radiation creep, hardening, embrittlement, physical property changes (such as reduced thermal conductivity) phase changes and possibly reduced precipitate stability. Even in a steady state machine, changes in power levels could lead to concerns with low cycle fatigue, and issues relating to coolant compatibility and tritium permeation and inventory may be important.

The plasma facing materials such as first wall and divertor components have most of the preceding requirements plus additional requirements for plasma compatibility. These include things such as sputter erosion, outgassing, hydrogen recycling, and possible low atomic number. These materials may also be required to absorb large amounts of energy in very short times during off normal events and will be exposed to high surface heat fluxes during normal operation.

The primary candidate toaterials considered for a TIBER II type device are stainless steel for the blanket and carbon, beryllium and tungsten-clad copper for the plasma facing components. Other materials sometimes mentioned include aluminum alloys and ceramics, 
materials which have the advantage of being low activation. The fabrication of these components is also a critical area, with joining techniques such as welding and brazing central to many designs, as is the reliability of mechanical fasteners. Virtually all interior components will have to be replaced during the reactor lifetime, and, hence, compatibility with remote maintenance is required.

For the TIBER $\Pi$ divertor, the design consists of $2 \mathrm{~mm}$ thick tungsten tiles attached to water cooled copper tubes with an inside diameter of $15 \mathrm{~mm}$. For tungsten with its high atomic number (high-Z) to work as a divertor tile material, a low edge temperature (about $10 \mathrm{eV}$ ), high recycling operational regime is required to completely suppress sputtering of tungsten for the entire plasma duration. Even small fractions of a percent of tungsten in the plasma can significantly reduce nuclear yield. The degree that the divertor chamber is isolated from the plasma can also be an issue, since redeposition of sputtered tungsten on the first wall can also lead to plasma contamination through sputtering from charge exchange neutrals. If ungsten tiles can be made to work, they will provide a divertor with a longer design life than the beryllium and carbon alternatives discussed later. Tungsten has been considered in other tokamak designs. For example, the $\mathrm{NET}^{9}$ and $\mathrm{INTOR}^{10}$ designs have at various stages included ungsten divertor tiles. The choice of tungsten as a divertor material for TIBER II is currently in the category of a risk with a potentially high return. Results from experiments and research with tungsten must be considered prior to beginning the final ITER/TIBER design.

Alternative divertor tile materials for TIBER II include beryllium and various forms of carbon. Beryllium, although much lower $\mathbf{Z}$ than tungsten, swells due to an $\mathbf{n}$-alpha reaction which shortens its useful life. One problem with beryllium, toxicity, is less significant in the D-T fusion environment since special handling of activated materials would already be necessary. Experiments with a beryllium limiter in ISX-B 11 showed it to be useable for high-heat-flux applications. Ductility, a problem at room temperature, is significantly higher at the $300-500^{\circ} \mathrm{C}$ operating temperature. Beryllium also serves as an oxygen getter, lowering oxygen impurity levels in the plasma. The Joint European Torus (JET) is considering further experiments with 
beryllium belt limiters. If these experiments are conducted, the information will be useful in selecting the final ITER/TIBER design.

Various forms of carbon are also candidate limiter tiles. Pyrolytic graphite has very high thermal conductivity, approaching or exceeding the conductivity of copper. In a neutron environment, however, the highly ordered structure of the graphite is quickly destroyed, along with the strength and conductivity. For this reason, its use as a divertor tile material was not considered. Carbon-carbon composites offer improved toughness over nuclear grade graphites but the lifetime of these composites in a neutron environment is also in question, as discussed in the section on first wall materials. Graphite tiles are a possibility, but as discussed later in this section, joining of graphite to a copper substrate in a neutron environment also presents problems.

The substrate and coolant channel for the TIBER divertor was chosen to be alumina dispersion stiengthened copper such as Glidcop A1-15 from SCM metals. This copper offers high yield strength (350-400 MPa), and retains good strength properties at temperatures as high as $500^{\circ} \mathrm{C}$. An alternative substrate material is OFHC copper, which exhibits an order of magnitude lower yield strength than the alumina dispersion strengthened copper. OFHC also loses strength rapidly, so that its use is usually limited to temperatures below $350^{\circ} \mathrm{C}$. Amzirc, a relatively high strength copper alloy was rejected because it tends to overage with long exposures to high temperatures and lose its strength.

A major material issue in the TIBER divertor design is the joining of the tiles to a copper substrate. The joint in the TIBER divertor must secure tiles subjected to both high heat fluxes, 6$7 \mathrm{MW} / \mathrm{m}^{2}$, and significant neutron fluence, 1-3 MW-yr $/ \mathrm{m}^{2}$. Potential problems are high residual stresses attributable to thermal expansion mismatch between the tile and the substrate, and differential swelling of the tile and substrate when subjected to a neutron flux. When a low thermal expansion material such as tungsten is brazed to a high thermal expansion material such as copper, residual thermal stress are locked into the joint during cooling following the braze. Reducing the tile size reduces the differential strain and resulting stress, but many small (about 1 
$\mathrm{cm}$ square) tiles would be required to bring stress to an acceptable level 12 . Another alternative is to introduce a pliable interlayer such as a sponge metal or dead soft copper to permit creep of the interlayer during cool down to reduce the stresses. Testing to the design heat flux level of the joint selected would be conducted prior to installation on IFERTIBER. Such testing would determine the design life without neutron fluence, and would determine if the high strength tungsten tile caused significant damage in the softer copper substrate after a high number of thermal cycles. Such damage has been observed in laboratory testing with an electron beam heat source ${ }^{13}$. Previous work on joining tungsten to copper9,it have used direct bonds with molten copper wetting the tungsten surface and nickel-based braze alloys. The joint presented in Reference 9 was tested to the $2 \mathrm{MW} / \mathrm{m}^{2}$ level, which is lower than the present TIBER peak heat flux level of 6-7 MW/m².

Beryllium joining is much easier because the thermal expansion coefficients of beryllium and copper are a close match. Since beryllium forms alloys with copper, it may be possible to develop a design in which the beryllium tiles are bonded directly to the copper substratc without a braze material. One concem with beryllium is that the swelling rate from neutrons will be higher than copper, leading to stresses at the joint, and potential failure of the joint.

Joining of carbon materials to a copper substrate combines both of the problems discussed above. Carbon, with its low thermal expansion provides a poor expansion match to metal substrates such as copper. One alternative is to consider a design in which a high expansion graphite such as POCO AXF-5Q is matched to a low expansion meral such as molybdenum. When subjected to a neutron flux, carbon first shrinks and then swells, leading to potential joint failure.

Another maierial concern in an actively cooled divertor is corrosion and erosion leading to failure of a coolant tube. The present design is based on a cooling water flow of $8 \mathrm{~m} / \mathrm{s}$. This flow velocity is much higher than that employed in more conventional cooling applications and can lead to a combined corrosion/erosion phenomenon if not carefully checked. In the corrosion/erosion process, corrosion products are eroded from the wall which exposes bare

$$
\text { B - } 11
$$


copper which is then subject to corrosion. In some cases this has led to rapid tube failure. Outof-core testing with simple flow loops or further analyses will be necessary before the design is confirmed. Another concern is radiolytic corrosion caused by particle interactions which has been observed 15 in the water circuits of high-energy particle accelerators. In this case, irradiated water produces $\mathrm{OH}$ radicals which combine to produce $\mathrm{H}_{2} \mathrm{O}_{2}, \mathrm{H}_{2} \mathrm{O}^{-}$radicals, and $\mathrm{H}_{2}^{+}$ions which in turn cause corrosion of copper surfaces. While this effect is present in high-energy particle accelerators, the necessity for maintaining low conductivity water prevents introduction of $\mathrm{pH}$ control and oxygen scavenging chemicals such as hydrazine. This simple addition to the divertor cooling water may be adequate to suppress the corrosion, but further evaluation during final design will be necessary.

For the TIBER II first wall armor, replaceable $1 \mathrm{~cm}$ thick carbon-carbon composite tiles bolted to the first wall were selected. Carbon-carbon composites are a wide class of materials made with a variety of techniques. For fusion applications, this class of materials offers the following advantages:

- High strength and toughress when compared to graphite and other carbon materials

- Low atomic number so that plasma contaminarion attributable to material erosion is minimized

- Useable to temperatures of more than $2000^{\circ} \mathrm{C}$

- High electrical resistivity minimizes eddy current forces during disruption. transients

- High emissivity (at least in the original state) permits radiative cooling schemes. Carbon-carbon composites are made from at least three types of fibers: pitch, polyacrylnitride (PAN), and rayor, with various yams and weaves. Depending on the strength and properties desired, fibers may be woven in $2,3,4$ or more directions before the resulting fabric is impregnated with resin or pitch and densified at high temperatures. Often several stages of impregnation and densificaiton are recuired to achieve the desired final material density. The methods used to densify the materials vary, and are often proprietary. Because of the variation in manufacturing methods, some composites will be better suited for specific applications than 
others, and generalizations about the class of materials must be made carefully. Costs of carboncarbon composites also vary widely, adding further complexity to the selection of an appropriate material. A general discussion of carbon-carbon and other composite structures may be found in reference 16.

On the negative side, the major question with the use of carbon-carbon composites in TIBER II is the susceptibility carbon fibers have shown to neutron damage. Tests in fission reactors 17,18 with carbon cloths and fibers have shown that general degradation of material properties occurred much faster in the cloths and fibers tested than in nuclear grade graphites. Extrapolation of these results suggests that carbon-carbon composites will also have poor neutron radiation resistance. At present, research 19 is underway to determine if radiation resistant carbon-carbon composites can be made. The fibers now being evaluated for use in radiation resistant carbon-carbons include meso phase pitch (P-100) fibers and specially made chemical vapor deposited (CVD) fibers. Lower cost, readily available alternative fibers will also be evaluated as part of the study. The study will continue through 1989.

One side effect of making the radiation resistant carbon-carbons is that the radiation resistant fibers have a high electrical conductivity, approaching that of metals. This means that at least initially, radiation resistant carbon-carbons may be subject to increased disruption force loads. As the neutron damage increases during operation, the electrical resistivity will increase, lowering the disnuption forces.

The present goal is to use specially selected fibers to build carbon-carbon composites with the neutron irradiation lifetime equivalent to that of nuclear grade graphites. Even if this goal is reached, the first wall tiles for TIBER II will still require several changes over the life of the device. Current graphites are estimated to be good for approximately $1 \mathrm{MW}-\mathrm{yt} / \mathrm{m}^{2}$ of neutron fluence at typical wall conditions. This would result in 3 to 4 required changes for a total wal! fluence of 3 to $4 \mathrm{MW}-\mathrm{yr} / \mathrm{m}^{2}$.

The general plasma interaction behavior of carbon-carbon composites does not seem to be a problem. A multi-directional weave carbon-carbon composite head has been operating

$$
B-13
$$


successfully for about a year on th: ALT-I experimental limiter at the TEXTOR tokamak in Juelich, FRG. Plasma performance has been as good as with previous all-graphite heads. No special conditioning of the head was required to achieve good plasma performance. TFTR and JET have also both recently installed composite structures that will give further experience in this area.

Alternative wall armor choices for TIBER II are graphite and beryllium. Tungsten, the choice for the divertor tiles, is ruled out as a first wall armor because sputtering from charge exchange neutrals would lead to unacceptable impurity levels of tungsten ir the plasma. Graphite would have many of the advantages of a carbon-carbon composite, but with much lower strength and toughness with a consequent higher risk of tile failure. Costs of graphite armor tiles would also be lower than composites.

Beryllium armor tiles have the additional advantage, besides being a low- $Z$ material, of acting as an oxygen getter. This would lead to improved plasma performance through lower

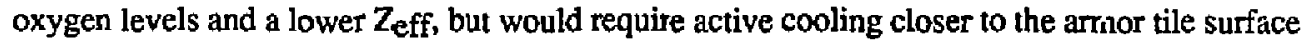
because beryllium has a lower melting point, and the high temperatures (about $1500^{\circ} \mathrm{C}$ ) planned for graphite armor would not be achievable.

In summary, the materials problems for steady state fusion devices are formidable. Devices without high neutron fluxes such as ATF-SS can probably be successfully cperated using existing metal alloys for the structure and graphite for plasma facing components. Next generation machines such as TIBER II will require neutron damage resistant materials for successful operation, and, hence, this additional requirement will further complicate materials problems. 
Table 1. Desirable Characteristics for High Heat Flux Materials

\begin{tabular}{|c|c|c|c|}
\hline Characteristic & $\begin{array}{l}\text { Desired } \\
\text { value }\end{array}$ & $\begin{array}{l}\text { Strongly affected } \\
\text { by radiation }\end{array}$ & $\begin{array}{l}\text { Response } \\
\text { affected }\end{array}$ \\
\hline Thermal conductivity & high & yes (C, ceramics) & thermal stress \\
\hline Thermal expansion & low & no & " \\
\hline Young's Modulus & low & no & \\
\hline Electrical conductivity & low & תо & eddy currents \\
\hline Specific heat & high & no & $\begin{array}{l}\text { transient } \\
\text { heating }\end{array}$ \\
\hline Yield Strengthin & high & yes & \\
\hline Ductility & high & yes & \\
\hline Creep & low & yes & $\begin{array}{l}\text { mechanical } \\
\text { design }\end{array}$ \\
\hline Fatigue crack growth & low & no & \\
\hline Frachire toughness & high & yes & \\
\hline Swelling & low & yes & \\
\hline Corrosion & low & no & $\begin{array}{l}\text { coolant } \\
\text { interactions }\end{array}$ \\
\hline Activation & Iow & yes & $\begin{array}{l}\text { maintenance, } \\
\text { disposal }\end{array}$ \\
\hline Sputtering & low & no & erosion, \\
\hline Atomic Number & low & no & impurity source \\
\hline $\begin{array}{c}\text { Tritium retention } \\
\text { permeation }\end{array}$ & low & $?$ & safety,operation \\
\hline
\end{tabular}




\section{References}

1. J.B. Whitley, J. Nucl. Mater. 133 and 134 (1985) 39-45.

2. W.B. Gauster, J.A. Koski and R.D. Watson, J. Nucl. Mater. 122-123 (1984) 80.

3. G.L. Kulcinski, Plasma Physics and Controlled Nuclear Fusion, Vol. II, IAEA-CN-33/S3-1 (1974) 251.

4. R.W. Coun, J. Nucl. Mater. 103-104 (1981) 7.

5. D.L. Smith, J. Nucl, Mater. 103-104 (1981) 19.

6. R.E. Nygren, J. Nucl. Mater. 103-104 (1981) 31.

7. R. Behrisch, J. Nucl. Mater. 85-86 (1979) 1047.

8. G.M. McCracken and P.E. Stott, Nucl, Fusion 19, No. 7 (1979) 889.

צ. F. Brossa, G. Federici, V. Renda, and L. Papa, "Experimental Testing and Theoretical Analysis of Samples of a Divertor Plate for NET," in Proceedings of the 11th Symposium on Fusion Engineering, Austin, Texas, 1985, pp. 928-931.

10. J.N. Brooks, et al., INTOR - Phase II, Part 3, Report EURFUBRU/XII-52/86/EDV22, 1986.

11. P.H. Edmonds, R.D. Watson, and M.F. Smith, "Design and Eabrication of a Beryllium Limiter for ISX-B," in Proceedings of the 13th Symposium on Fusion Technology Sept. 2428,1984 , Varese, Italy.

12. J.P. Blanchard and R.D. Watson, "Residual Stresses in Bonded Armor Tiles for In-Vessel Fusion Components," Nucl. Eng. Design_Fusion. vol. 4, 1986, pp. 61-66.

13. A.W. Mullendore, J.B. Whitley, and D.M. Mattox, "The Development and Laboratory Testing of Low Z Refractory Coatings for Fision Reactor Limiters," J. Nucl. Mat., vol. 93 \& 34, 1980, pp. 486-492.

14. T. Horie, M. Seki, A. Minato, and T. Tone, "Experimental Study for Tungsten-Copper Bond of a Divertor Plaie," in Proceedings of the 11th Symposium on Fusion Engineering, Austin, Texas, 1985, pp. 937-940. 
15. F. Hoyer, M. Bourges, and R. Deltenre, "Radiolytic Corrosion and Related Problems in the Cooling Water Circuits of High Energy Particle Accelerators," CERN Report 68-2 DIHP/MSC, Jan, 25, 1968, Geneva.

16. L.E. McAllister and W.L. Lachman, "Multidirectional Carbon-Carbon Composites," in Fabrication of Composires, A. Kelly and S.T. Mileiko, editors, North-Holland, Amsterdam, 1983, pp. 109-175.

17. W.J. Gray, "Neutron Irradiation Effects on Carbon and Graphite Cloths and Fibers," Report BNWL-2390, Battele Northwest Laboratory, Hanford, Washington, August 1977.

18. R.J. Price, G.R. Hopkins, and G.B. Engle, "Effects of Fast Neutron Irradiation on Carbon Fibers," in Extended Abstracts and Program of the 17th Biennial Conference on Carbon, University of Kentucky, Lexington, June 16-21, 1985, p. 348.

19. G.B. Engle, NAMCO, Inc., San Diego, "Neutron Irradiation Stable Carbon-Carbon Composites," Contract DE-AC03-86ER80418, May 15, 1987 - May 15, 1989. 


\section{Thermal Stress and Fatigue}

The thermal stresses that develop when materials are exposed to high heat fluxes are often the most severe loads to which the material is subjected. Temperature gradients during the steadystate removal of a heat flux of $10 \mathrm{MW} / \mathrm{m}^{2}$ range from approximately $25^{\circ} \mathrm{C}$ per millimeter for copper to values over $1000^{\circ} \mathrm{C}$ per millimeter for stainless steel. If the component has constraints that resist the expansion of the heated area, then thermal stresses will develop, with the stresses during the heating cycle being compressive in the near-surface region and tensile in the bulk region. 1

A figure-of-merit describing the stress response of a material to an incident heat flux can be derived in a straightforward manner. Since the temperature gradient produces the thermal stress, the first step will be to develop a relationship for the temperature profile during surface heating. Neglecting the temperature dependence of the thermal cond:ctivity $\kappa$, and separation of $d$ and a temperature at the coolant channel of $T_{d}$, the temperature profile during steady state operation is given by a rearranged form of Fourier's Law of heat conduction 2 :

$$
T(x)=T_{d}+\frac{F(d-x)}{x}
$$

If the material is assumed to be fully constrained (by, for example, the cold material behind the coolant channel), then the thermal stress at the surface, $\sigma_{t h}$, is given by ${ }^{3}$;

$$
\sigma_{\mathrm{th}}=\mathrm{E} \alpha \Delta \mathrm{T} /(1-\mathrm{v})
$$

where

$\alpha=$ coefficient of themal expansion, $E=$ Young's Modulus, 
$v=$ Poisson's ration

The temperature gradient $\Delta T$ can be found from Eq.1. For the case of a steady state solution with an applied constraint that allows expansion but no bending, the stress profile will be linear, passing through zero near the center. Since only half the component is under a particular stress state (e.g., compressive in the halî inear the heated surface), then only half the total temperature gradient across the component enters into the stress calculation, i.e.:

$$
\Delta \mathrm{T}_{\text {effective }}=\mathrm{F} \mathrm{d} / 2 \mathrm{~K} \text {. }
$$

If the thermal stress is now set equal to the yield strength $\sigma_{\mathrm{y}}$ of the material and the equation is then solved for $F$, the thermal stress heat flux limit is derived:

$$
F_{0}^{\text {stress }}=2 \sigma_{y}(1-v) \kappa / d \alpha E
$$

This limit is shown plotted as a function of $d$ for copper in Fig.1. For copper, this maximum stress limit for a one centimeter thick structure corresponds to a heat flux of less than $10 \mathrm{MW} / \mathrm{m}^{2}$. The limits for a large number of different materials are compared in Fig.2. The values for the copper alloys are high due to their high thermal conductivities and relatively high strengths, while the high value for graphite is due to its high strength and low thermal expansion. The low value for stainless steel is due mainly to its very low thermal conductivity.

If the heat flux limit given by Eq.4 is not exceeded, then the component deformation from the heat pulse will be primarily elastic. The fatigue resistance of most materials under these conditions is adequate and will probably not be life limiting. It is quite common, however, for high heat flux components to be designed to operate under conditions that cause the thermal stress limit to be exceeded. In such a case, a portion of the surface of the material will be plastically deformed by the thermal cycling. As an example, a finite element calculation for a 


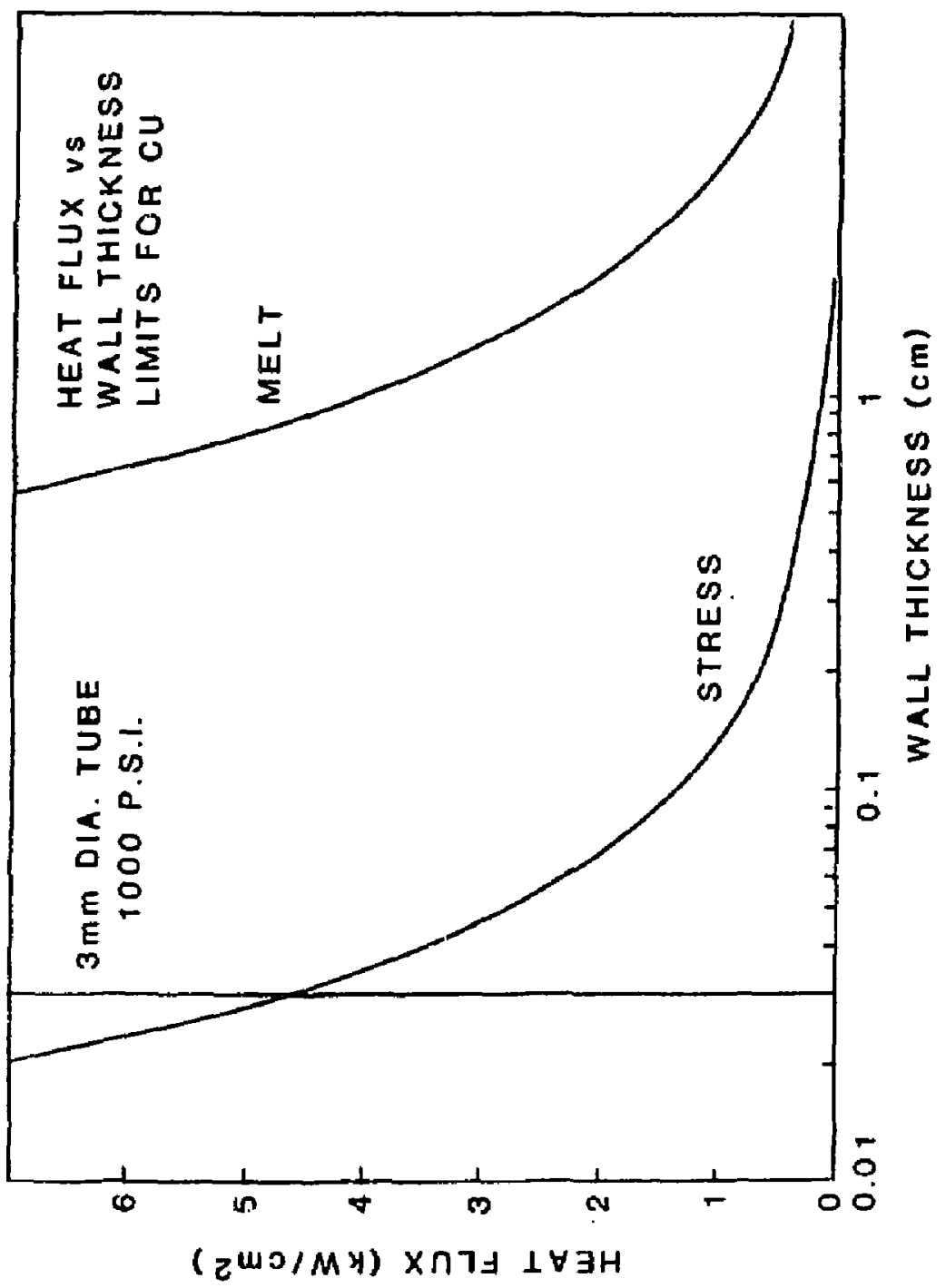

Fig.1. A plot of the surface thermal stress limit for copper. Also shown is the surface melting limit and the minimum thickness required to withstand a $1000 \mathrm{psi}$ coolant. 


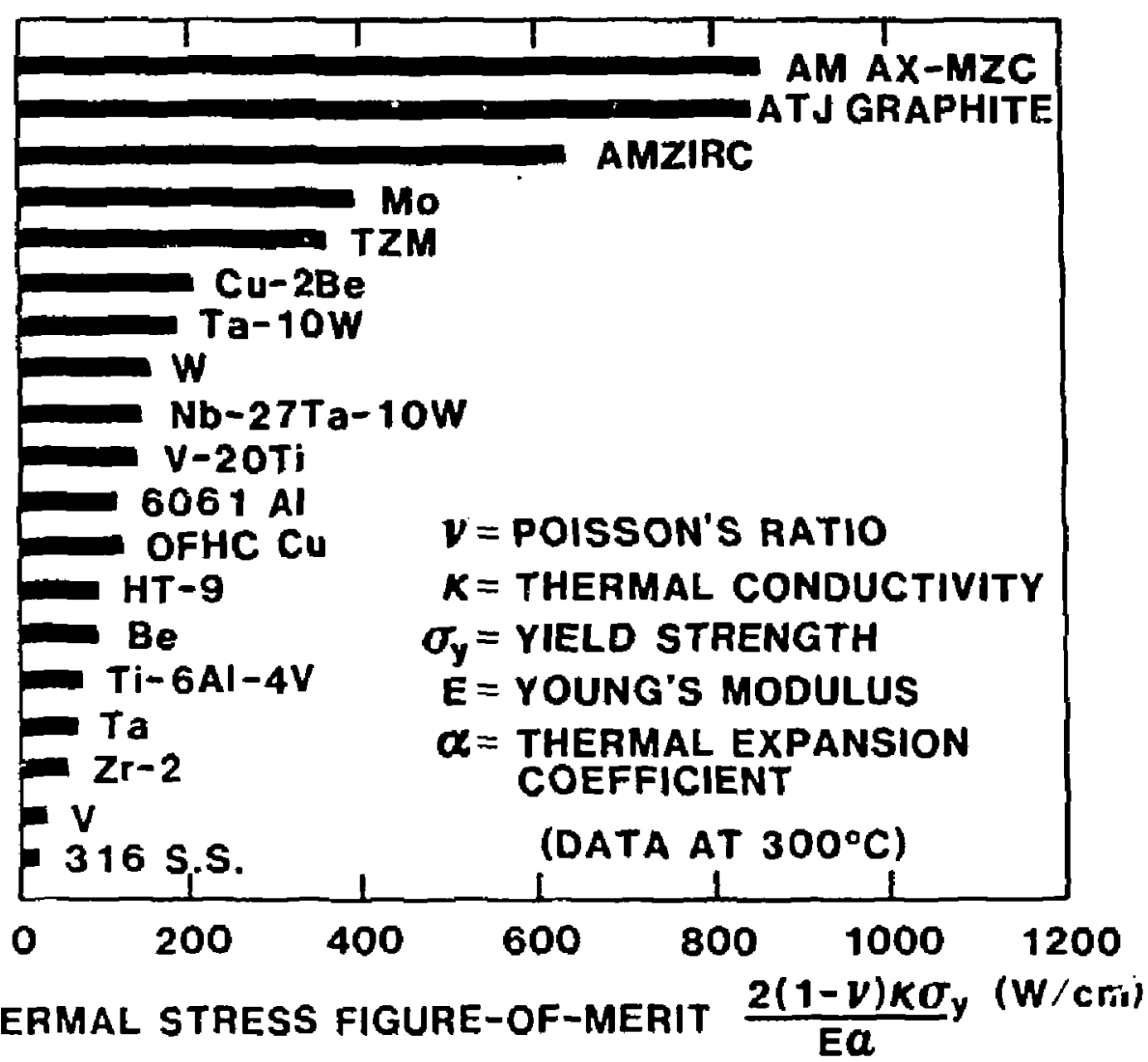


beryllium limiter exposed to a surface heat flux of $2.5 \mathrm{MW} / \mathrm{m}^{2}$ for a pulse length of $15 \mathrm{~s}$ is shown in Fig.3. Note that a purely elastic analysis predicts surface stresses well in excess of the material's yield strength. Since the yield strength is temperature dependent, it decreases in the near surface region where the temperature is rapidly increasing. The zone of plastic deformation extends to a depth of about 2 to $3 \mathrm{~cm}$. The stress profile for this case at a time $40 \mathrm{~s}$ after the beginning of the heat pulse (i.e., after $15 \mathrm{~s}$ of heating followed by 25 of cooling) is shown in Fig.4. The plastic defomation that occurred during the heating phase is seen to lead to a residual tensile stress in the surface region during cooling. This complex stress/strain history is one reason why a component subjected to repeated thermal loading will require extensive thermal testing before lifetimes can be reliable predicted.

In summary, thermal stress is a rather simply defined problem with often complex results. It is a major concern in many components and may be a problem even in relatively low heat flux areas, such as first wall structures where stainless steel is used. The relaxation of thermal stress by creep or plastic deformation can lead to complex stresses when the heat load is removed and must be carefully analyzed and tested, especially in complex composite structures.

\section{References}

1. J.F. Schivell, D.J. Grove, J. Nucl. Mater. 53 (1974) 107.

2. A.J. Chapman, Heat Transfer (Macmillan, New York, 1984).

3. S. Timoshenko and J.N. Goodier, Theory of Elasticity (McGraw-Hill, New York, New Yark, 1951). 

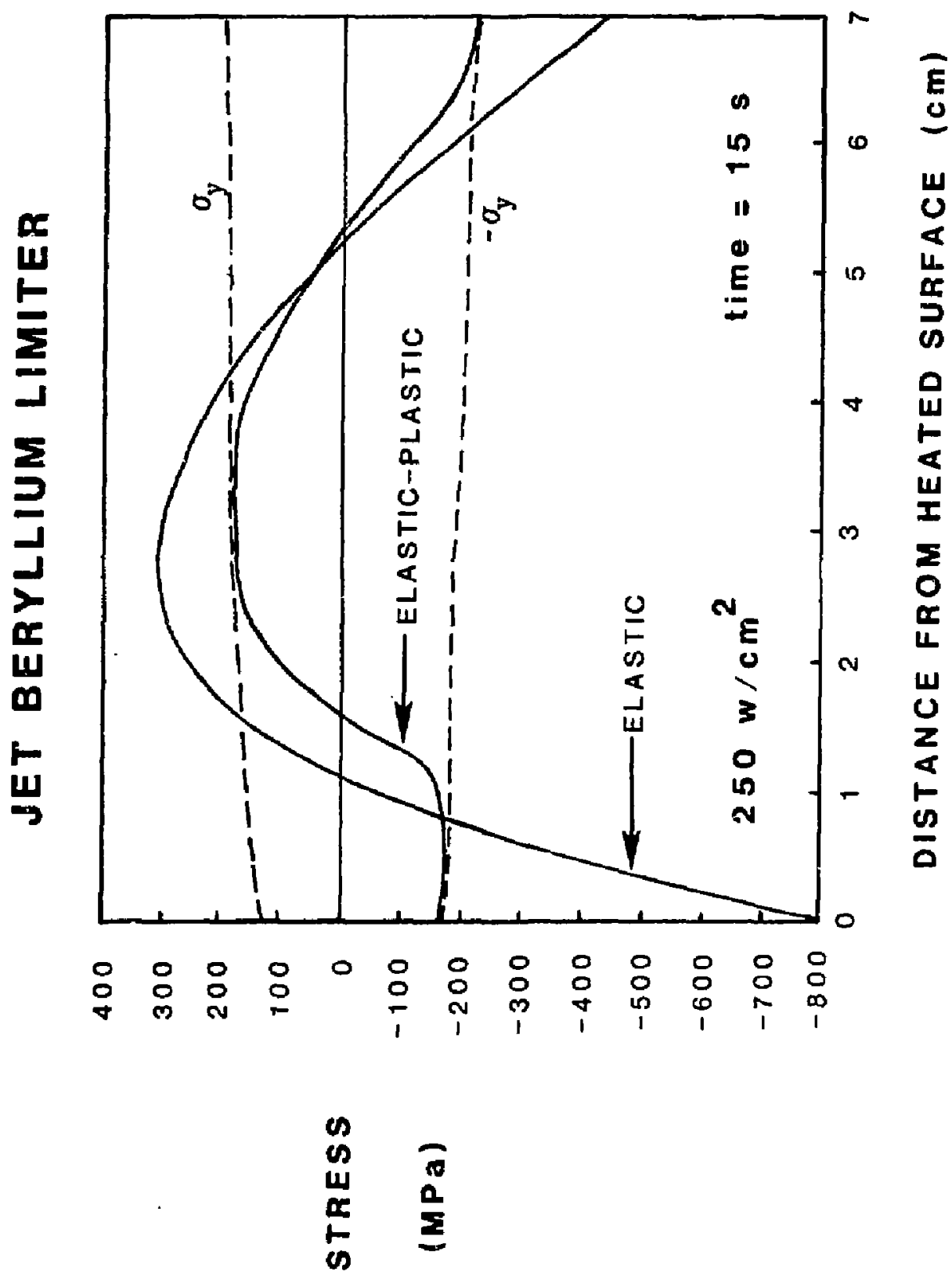

Fig.3. A finite element calculation of the thermal stress profile in a $7 \mathrm{~cm}$ thick beryllium limiter segment exposed to a surface heat flux of $2.5 \mathrm{MW} / \mathrm{m}^{2}$ for $15 \mathrm{~s}$. Both the purely elastic calculation and the elastic-plastic calculation are shown along with the yield strength for beryllium appropriate for the temperatures calculated by the code as a function of depth. 

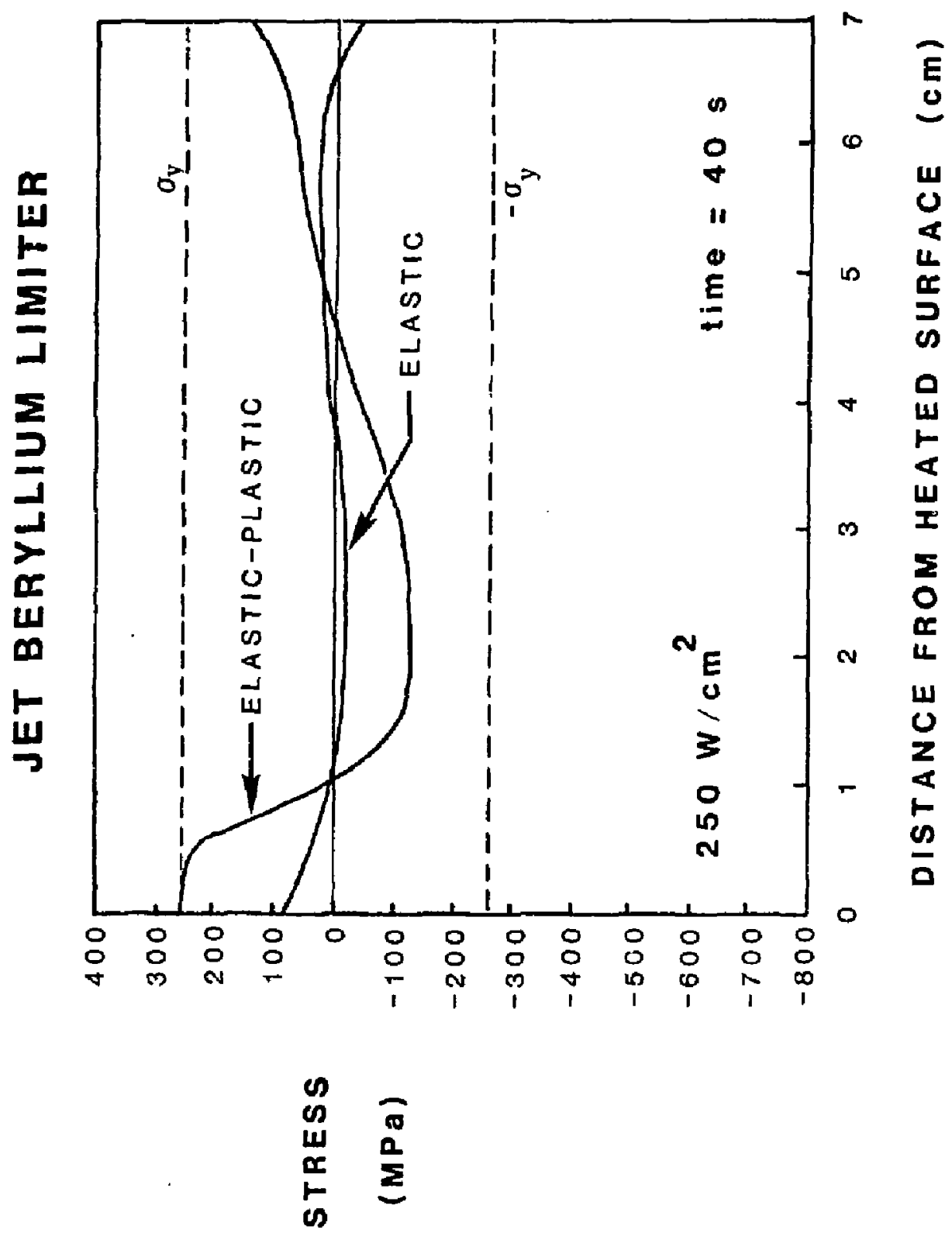

Fig.4. A finite element calculation for the tile of Figure 3 after a $25 s$ cooling period. Note the residual tensile stress at the surface shown by the elastic-plastic calculation. This residual stress is caused by the compressive yielding during the hearing phase. 


\section{Heat Transfer}

Active cooling of high heat flux fusion reactor components requires the use of some demonstrated but not yet well documented or analyzed cooling regimes. For example, subcooled flow boiling, which is described in more detail below, can easily accommodate heat fluxes of 10$100 \mathrm{w} / \mathrm{cm}^{2}$ [1], but most of the work done with this phenomenon has concentrated on heating patterns and geometries which are applicable to fission reactors, such as circumferentially heated circular tubes. In fusion power applications heat fluxes tend to be applied to only one side of the component, and thus primarily to one side of the cooling channel. This type of heat flux pattern can have a significant effect on the applicability of existing heat transfer data.

To facilitate further discussion the different flow regimes possible with liquid coolants flowing in a heated channel will be reviewed briefly. Fig. 1 traces the effect the different flow regimes have on heat transfer. The abscissa represents the difference between the temperature of the heated wall and the bulk or average temperature of the fluid, while the ordinate represents the heat flux supported by that temperature difference. At the lower left, heat transfer with fully developed turbulent flow can be predicted with the well known Dittus-Boelter ${ }^{2}$ or Colburn ${ }^{3}$ correlations. Corrections to these correlations for heat fluxes primarily on one side of the cooling channel also exist in the literature ${ }^{4}$. As the wall temperature, and thus $\left(T_{w}-T_{b}\right)$, is increased the liquid near the wall exceeds it saturation temperature; and if wall surface conditions permit, vapor bubbles are nucleated at the wall. Because the average fluid temperature, $T_{b}$, is still below the saturation temperature, the bubbles which are formed at the wall collapse after mixing the cooler fluid at the center of the channel. One effect of this bubble nucleation, mixing, and collapse is a significant increase in the heat flux which can be passed to the liquid.

Correlations predicting the onset of nucleate boiling for water in simple geometries are available, ${ }^{5}$ but effects such as wall surface conditions and dissolved gases in the water can affect the accuracy of the predictions. Correlations predicting the heat fluxes supported in this region of highly subcooled flow boiling are sparse ${ }^{6,7}$ for the geometries of interest in fusion power 

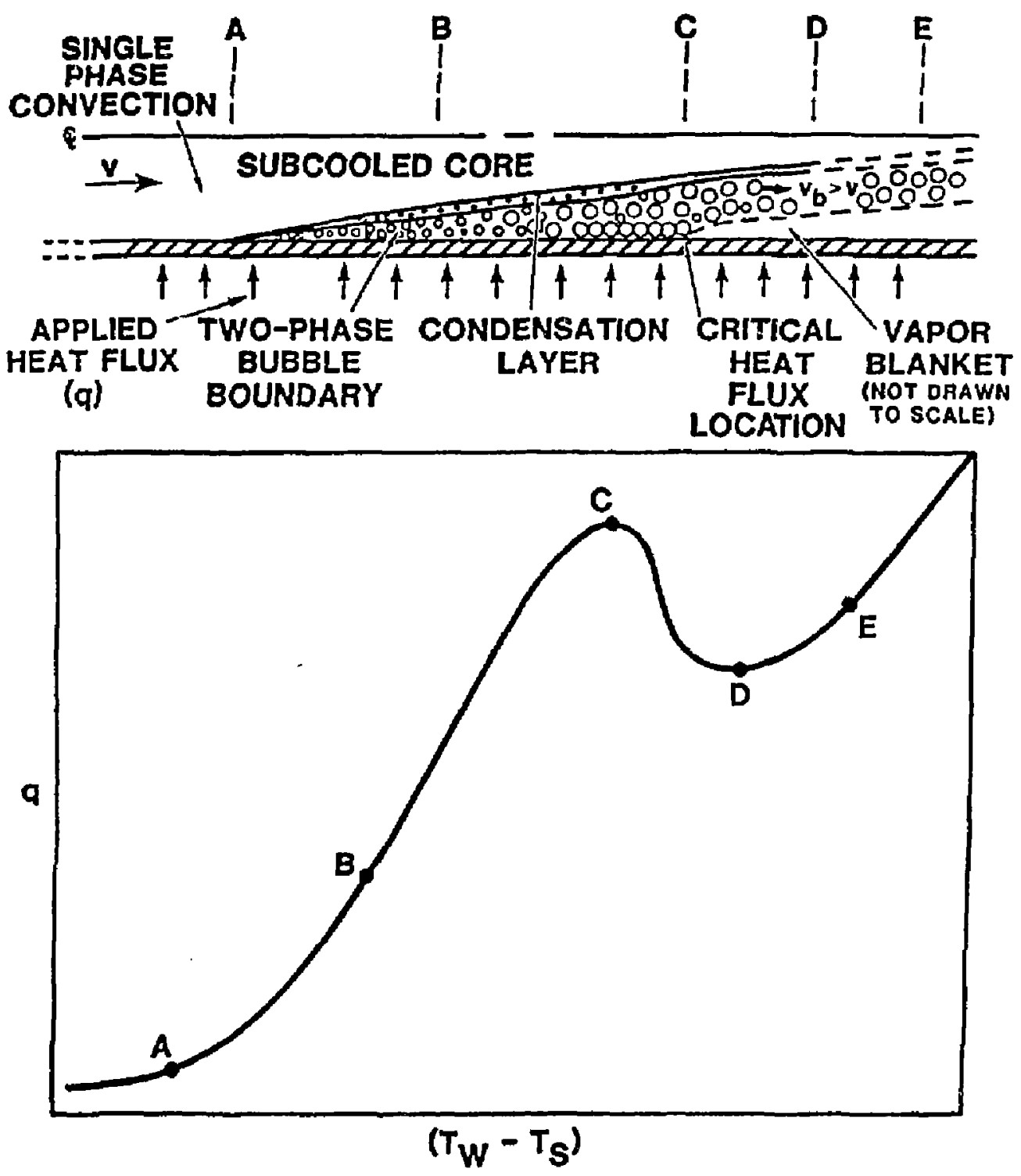

Fig.1. Flow regimes in cooling channels: heat flux from the wall to the coolant plotted against the difference between temperanires of the heated wall and the bulk of the coolant fluid. 
applications. However, this method has been successfully used in neutral beam dumps at Lawrence Berkeley Labs ${ }^{8}$ and Oak Ridge National Laboratory ${ }^{9}$ to remove steady-state heat fluxes of 2 and $7 \mathrm{~kW} / \mathrm{cm}^{2}$, respectively.

As wall temperanures continue to rise, nucleate boiling activity continues to increase. If the heat flux at the cooling channel wall is too high, the mixing of the bubbles with the central cores of even a highly subcooled fluid is not rapid enough, and vapor accumulates on the wall. If the vapor with its poorer heat transfer properties blankets a significant portion of the wall, heat transfer is significantly reduced, and a phenomenon known as critical heat flux occurs in which wall temperatures must substantially increase to maintain the previously supported heat flux through the vapor blanket. If this temperature rise is sufficient to melt the thin-walled tube, then "burnout" and failure can occur at the location of critical heat flux. Prediction of this phenomenon, represented as the peak heat flux in Fig.1, has been the subject of considerable research which has been recently surveyed by Boyd 10 .

At the upper right of the curve in Fig. l, the steady state film boiling regime is entered. Here an annular ring of vapor at the cooling channel wall passes the heat flux to a central core of liquid. Because of the extremely high wall temperatures required, this mode of operation is generally avoided. The relative simplicity of film boiling compared to nucleate boiling does permit a more complete analysis of this flow regime ${ }^{1}$, however.

For fusion reactor applications, the highly subcooled flow boiling regime offers great promise for many applications. As mentioned, heat transfer correlations and experimental data in this regime are very limited. This sparsity of data is due to two causes; first, industrial applications for this flow regime have previously been limited, and, second, the complex heat and mass transport processes involved are not easily analyzed.

To make use of highly subcooled flow boiling in the near term, an approach similar to that employed by Kays and London 12 will be necessary. These authors correlated heat transfer data in a useable format for a wide range of useful but hard-to-analyze heat exchanger configurations. Experimental results obtained from instrumented heat exchangers were used to 
set parameters in performance prediction equations. Facilities such as the PMTF can be used to evaluate subcooled flow bojling in an analogous manner.

Several schemes have been proposed to enhance the performance of cooling channels exposed to high heat fluxes. Some schemes, such as twisted metal tapes inserted lengthwise in circular tubes ${ }^{13}$ and spiral grooves cut into the inner tube wall ${ }^{14}$ enhance the mixing of fluid near the tube wall with the cooler surrounding fluid. This type of mixing is especially useful with the one-sided heat flux patterns encountered in most fusion power afplications. Other heat transfer enhancement schemes include placing pins and fins in the coolant passages ${ }^{8}$ and tangentially injecting colder liquid into the flow ${ }^{15}$. In the latter scheme a second parallel coolant channel is provided to inject liquid tangentially at various locations along the heated tube. Weede and Dhir 15 have reported significant increases of as much as $34 \%$ in the measured critical heat flux. All of these schemes have been demonstrated in a prototype or experimental jorm, but predictive correlations snd data are not yet widely available.

If high velocity flows with subcooled boiling are used for prolonged periods, erosion of the coolant channel material by processes similar to cavitation damage in pumps and propellers may cause premature failure of actively cooled components ${ }^{16}$. The magnitude of this problem will depend on the details of the design and heat load.

For many industrial processes water is the preferred cooling medium because of its availability, non-toxicity and heat transfer properties. For fusion applications treated water also has the advantage of low electrical conductivity which tends to minimize problems with eddy currents and electromagnetically induced forces. In addition, most boiling heat transfer ressarch to date has concentrated on water, making it one of the few well- characterized boiling heat transfer fluids available.

Liquid metals, such as sodium lithium, lithium-lead mixnures, and molten salts have been suggested as heat transfer fluids for fusion applications. One significant problem with these fluids in the high heat flux environment is that their melting temperature is very close to the maximum allowed temperatures $\left(300^{\circ} \mathrm{C}\right)$ for materials such as copper 12 . For water, the 
saturation temperatures can be controlled so that the advantages of flow boiling heat transfer can be used without exceeding material temperature limits. For lower heat flux applications, such as first wails ${ }^{18}$ and blankets ${ }^{19}$, liquid metals and gases such as helium may be found to be useful as heat transfer media.

In summary, steady state devices will have to depend on some actively cooling method for heat removal, whether it be by direct cooling of a component or indirectly by radiation or conduction. There are several possible coolant media, each with advantages and disadvantages. The heat transfer data base needed for these fusion components is somewhat unique, but is gradually being filled in by test facilities.

\section{References}

1. F. Mayinger and B. Bucier, "Subcooled Boiling," in: Two-Phase Flow and Heat Transfer, Proceedings of the NATO Advanced Study Institute, August 16-27, 1976, Istanbul, Turkey, Eds. S. Kakac and T.N. Veziroglu (Hemisphere Publishing Corp., Washington, 1977) p. 581 .

2. F.W. Dittus and L.M.K. Boelter, "Heat Transfer in Automobile Radiators of the Tubular Type," Univ. of Calif. Publication Eng. 2, 443 (1930).

3. A.P. lbum, Trans. AIChE $\underline{29}, 174-210$ (1933).

4. W.M. Kays, Convective Heat and Mass Transfer (McGraw-Hill Book Co., New York, 1966), p. 174.

5. A.E. Bergles and W.M. Rohsenow, "The Determination of Forced Convection Surface Boiling Heat Transfer," Paper 63-HT-22, presented at 6th National Heat Transfer Conference of ASME-AIChE, Boston, Aug. 11-14, 1963.

6. W.H. Jens and P.A. Lottes, "Analysis of Heat Transfer Burnout, Pressure Drop and Density Data for High Pressure Water," Argonne National Laboratory, Report ANL-4624 (1951). 
7. J.R.S. Thom, et al., "Boiling in Suc-Cooled Water During Flow Up Heated Tubes of Annuli," Paper No. 6, Presented at the Symposium on Boiling Heat Transfer in Steam Generating Units and Heat Exchangers, Manchester, UK, Sept. 15-16, 1965, Inst. of Mech. Engrs., London.

8. G.D. Morgan, et al, MicDonnell-Douglas, MDC E2462, 1981.

9. C. Foster, private communications, Oak Ridge National Laboratory.

10. R.D. Boyd, in: Proc. of the 21st National Heat Transfer Conference, Seattle, 1983 (ASME, New York, to be published).

11. P.J. Berenson, ASME Jour, of Heat Trans. 83, 351 (1951)

12. W.M. Kays and A.L. London, Compact Heat Exchangers (National Press, Palo Alto, CA, 1955).

13. W.R. Gambill, et al., Chem. Eng. Prop. Symp. Ser. ㄱ7, 127 (1961).

14. M. Luv and A. E. Bergles, ASHRAE Trans. 85, 132 (1979).

15. J. Weede and V.K. Dhir in: Proc. of the 21st National Heat Transfer Conference, Seattle, 1983 (ASME, New York, to be published).

16. J.B. Whitley and R.S. Blewer, Performance and Failure Modes of Generic Beam Dump Panels, to be presented at the 10th Symp. on Fusion Engineering, Philadelphia, PA, Dec. 1983.

17. M. Abdou, private communication.

18. M. Abdou, et al., "Blanket and Selection Study, Interim Report," FED-INTOR/ICFW/8217, Oct. 1982.

19. M. Abdou, et al., Blanket and Selection Study, Interim Report," ANL/FPP/PM-177, August 198:. 


\section{Neutron Irradiation Effects}

Materials that can withstand the temperarures and thermal loads of HHF/PMI components may not survive even the unodest neutron irradiation doses that replaceable components will be exposed to over their lifetime in an ITER machine. Bulk irradiation effects are not expected to be significantly different for steady state or pulsed operation. However, average dose rates and total doses in ITER HHF/PMI components will probably be higher under steady state operation.

The vitally important issue concerning neutron irradiation effects in HHF/PMI materials, whether pulsed or not, is the severe lack of information on irradiation effects, especially for many of the present primary candidate materials. A modest scoping study of irradiation effects in potential HHF materials has been in progress. However, information is needed on mechanical and thermophysical property changes. A comprehensive plan for irradiation and testing of HHF/PMI materials for ITER and beyond needs to be developed and carried out.

\section{1) Neutron Irradiation Effects Issues}

The importance of neutron irradiation effects in HHF/PMI components in general has been pointed out in the studies and assessments of fusion technology such as the Technica] Planning Activity and the Finesse Study. An ad hoc Neutron Irradiation Planning Group has laid out the general issues involved in irradiation testing of HHF/PMI materials. Recent design efforts for near term devices (CIT, ITER) have identified irradiation effects in HHF/PMI materials to be a potentially critical issue.

\section{2) HHF/PMI Materials}

In general, materials choices for piasma interactive components are intimately linked to the specific component design. Thus, critical irradiation issues may change considerably from one design concept to another. The present candidate HHF/PMI materiais include carbon/carbon composites, graphite, beryllium, oxide dispersion-strengthened copper, metal matrix composites, 
and refractory metals and alloys. Near term requirements (for CIT and ITER) favor the use of graphite and $\mathrm{C} / \mathrm{C}$ composites. Actively cooled components, such as limiters, will probab!y have a low-Z material bonded onto a metal, e.g. graphite or $\mathrm{C} / \mathrm{C}$ composite brazed to copper. Other plasma-facing components, such as first wall armor, will rely on good thermal conductivity and toughness to withstand thermal shocks, e.g. C/C composites. Thus, the vital and unique radiation effects issues for HHF/PMI components have to do with interfaces--attachmert, bonding, and composite integrity--as well as the thermophysical properties of the individual materials.

Neutron effects data are available for many graphites, and limited data are available on refractories and beryllium, as well as some copper alloys. Neutron effects data are not available on carbon/carbon composites or some of the newer composite materials and alloys. There are essentially no data available for neutron effects on bonding for the materials of interest.

\section{3) Steady State Operation}

Nearly all research on irradiation effects in any materials for use in magnetic fusion reactors has dealt with steady state irradiations. The limited work on the effects of pulsed irradiation has been done primarily with ions in metals. This work has not revealed any critical differences in material behavior caused by pulsing that would be pertinent to ITER. Generally, the same things happen in pulsed and steady state irradiations of metals, but with different timing, depending on irradiation temperature, pulse lengths and dwell times, phase stability conditions, etc. A minor benefit of steady state operation is that possible effects of pulsing will not have to be accounted for in the fission reactor test facilities.

For primary candidate HHF/PMI materials in ITER pulsing is a secondary issue since irradiation effects in general are unquantified. 


\section{4) Irradiation Temperatures}

Choosing appropriate irradiation temperatures may be the greatest challenge in an irradiation program for HHF/PMI materials. Temperatures of HHF/PMI components could be in the range from $100^{\circ} \mathrm{C}$ to $2000^{\circ} \mathrm{C}$ or more, and in many components there will be steep temperature gradients. Irradiation effects are expected to be quite temperature sensitive in many materials. For example, in graphite the irradiation-induced swelling rate varies by an order of magnitude within this temperature range. Iradiations in the presence of a large temperature gradient will be difficult and expensive. Iradiations at a uniform high temperature will probably require the development of special irradiation fixtures.

\section{5) Neutton Energy Effects}

Fission reactors are the primary irradiation test facilities used in the fusion materials program. The large component of $14 \mathrm{MeV}$ fusion neutrons is missing in a fission reactor, so gas production and high energy cascade effects in a fission reactor are very different from a fusion reactor. Production of hydrogen and helium per dpa (displacements per atom) is one to two orders of magnitude less in fission reactors than in fusion reactors for most of the HHF/PM!I materials, including carbon, oxygen, copper (as well as for the major constituents of stainless steels). Beryllium is an exception, in that gas production differs by only about a factor of two. (Another special case is nickel, which has much higher helium production in a mixed-spectrum reactor such as HFIR than in a fusion reactor.) Gas production plays a major role in changes of mechanical properties and dimensional stability under some conditions. Until a high flux source of fusion neutrons becomes available, innovative techniques for studying effects of helium in HHF/PMI materials will have to be developed. One suggested technique is to substitutionally dope boron into graphite and $\mathrm{C} / \mathrm{C}$ composites. 


\section{6) Testing Requirements}

Neutron irradiation effects in HHF/PMI components may affect the integrity of a HHF/PMI component, its mechanical performance and its thermophysical properties. Post-irradiation testing must address all these possibilities.

Macroscopic and microscopic examinations of the material integrity must be made on representative interfaces and component configurations. Scanning electron microscopy, including various microprobe measuring techniques could be used.

Mechanical performance in service will be most affected by radiation-induced changes in fracture strength, strain-to-failure, fracture toughness, and creep properties. Post-irradiation mechanical test techniques exist that are suitable for most materials. Mechanical tests for composites may need to be developed. Dependence on temperature, dose and dose rate must be investigated.

A comprehensive program investigating dimensional stability under irradiation, including temperature and dose effects, needs to be established. A long range program should include examination of the microstructures in an effort to understand the nature of the dimensional changes.

Thermal conductivity and thermal expansion may change significantly under neutron irradiation for many materials. Degradation of thermal properties of graphite and $\mathrm{C} / \mathrm{C}$ composites is anticipated for neutron fluences less than the ITER lifetime dose. Maintaining good heat transfer coefficients over interfaces is also very important and should be examined as part of the testing program.

In general, post-irradiation testing of metals and alloys will have to be performed in a hot cell. Residual radioactivity in graphite and $\mathrm{C} / \mathrm{C}$ composites will probably be small enough to allow testing out of cell. In-cell handling and testing techniques are well developed. New test methods or apparatus may take somewhat longer to develop for in-cell use. 


\section{7) Present Status}

A progtam of irradiations in the FFTF is already in place for the scoping studies of the HHF task Group. Neutron doses relevant to HHF/PMI component lifetimes in ITER can be achieved in FFTF core positions in about one year. An irradiation program utilizing the HFIR is being planned for $\mathrm{C} / \mathrm{C}$ composites.

Iradiation testing is time consuming and expensive. The time required to accumulate dose levels can severely limit the number of iterations one might perform in development of a viable material. Post-irradiation tests are more difficult and expensive to perform if a hot cell is necessary.

Irradiation volumes are limited, which places restrictions on testing complex shapes, interfaces, etc. This introduces a particular challenge in the irradiation and testing of highly anisotropic materials, such as some composites. However, it should be possible to test inradiation effects on the bulk properties of most materials.

The data base on irradiation effects in the candidate materials is small, the number of important variables is large, and the time available for a comprehensive irradiation program is limited. More than ever, the reactor designers, materials vendors and materials researchers must work closely together throughout the development of these materials. 


\section{Erosion and Redeposition}

Erosion of the plasma facing components (PIC) in fusion devices is one of the most critical issues for the steady state operation. This is because erosion affects both the lifetime of the components in the machine, and contributes to the impurity content of the plasma. As the pulse length of present machines increases, it is not uncommon to find that the design of limiter and divertors structures is determined primarily by considerations of the erosion lifetime. In the event that the rapid erosion of some components determines the operational lifetime of the device, frequent maintenance and low machine availability must be accepted to repair and replace the plasma facing components. The physics of the plasma operation also depends very strongly on the impurity content in the plasma. The requirement of low $\mathbf{Z}$ wall materials in present pulsed machines, discussed in detail in the physics section of this report, illustrates the importance of minimizing erosion from an impurity production point of view for steady state devices. The erosion of the first wall, limiters and divertor surfaces will be one of the most critical problems for steady state operation.

The erosion of components in a tokamak occurs due the sputtering of the materials exposed to the plasma. Plasma bombardment of PIC surfaces produces physical sputtering, chemical sputtering, and radiation enhanced sublimation (which is a form of physical sputtering at high temperature and energies). The sputtered particles are released into the enge plasma with varying amounts of energy depending on the sputtering process. These particles can then be ionized in the edge plasma and transported into the center of the plasma or out to the tokamak boundary. If the source rate of the impurities is large, or the confinement time of the sputtered impurities is relatively long, the impurity level in the plasma can accumulate and eventually adversely affect the plasma performance. The impurity problem in tokamaks has led to the universal usage of low $Z$ materials for the PIC in present machines, and the use of renewable low $\mathrm{Z}$ coatings applied during glow discharges to reduce the sputtering of high $\mathrm{Z}$ materials from the first wall. 
Sputtering of the surfaces in the machine is produced not only by energetic hydrogen isotopes striking the surface, but also by the other impurities in the plasma which reach the PIC thorough normal plasma bombardment and via charge-exchange. Inevitably the surface erosion yield by the impurity atoms or ions far exceeds the yield from hydrogen sputtering. Significant quantities of impurity particles, such as oxygen, striking the surface can result in very high influxes of wall material into the plasma. In addition, the self sputtering yield of some materiats can exceed one at higher energies, also resulting in a large influx of wall material.

As will be discussed, the erosion yield of most materials for fusion applications is relatively well known from ion beam experiments. Recent laboratory plasma experiments have extended this sputtering yield data base to high incident ion fluxes and representative ion energies. The erosion of PIC surfaces has been estimated from these measurements to be large during steady state operation, and can exceed several $\mathrm{cm}$ per year for some high heat flux components. The major hope for prolonging the lifetime of PICs lies in the modification of the edge plasma conditions to reduce sputtering, or in redeposition. The effect of a modification of the plasma edge conditions on erosion will be discussed in part three of this section. Redeposition is the effect where the sputtered PIC material is ionized in the edge plasma and transported in the tokamak until it eventually deposits on the surfaces exposed to the plasma. The transport and mixing of materials in tokamaks is well known, and models are emerging which can predict the location of redeposition. The location of the redeposition and the nature of the material is presemly being studied in experiments in tokamaks and laboratory plasma devices. In steady state, the net erosion of PICs will be determined by the balance between erosion and redeposition.

\section{1) Erusion data base}

The sputtering data base for most materials of interest to fusion is well established, 1,2 and will not be reviewed here. The performance of low $\mathrm{Z}$ materials is of interest for this assessment, 
however, because these are the primary candidates for the ETR wall materials. A short summary of the most recent findings that affect steady state operation will be given and discussed.

The primary candidates for the PIC materials for the next generation machines are graphite, carbon-carbon composite weaves, beryllium, and some of the low $\mathrm{Z}$ carbides. The performance of graphite and beryllium in ion beam experiments and some tokamak experiments has been recently reviewed. ${ }^{3}$ Figure 1 shows the erosion data given by Roth ${ }^{3}$ for the energy and temperature dependence of carbon and beryllium sputtering. Beryllium oxide is found to have a significantly lower sputtering yield compared to clean $\mathrm{Be}$, due to chemically reduced physical sputtering. At higher temperatures $\left(650^{\circ} \mathrm{C}\right)$, pure Be from the bulk diffuses through the oxidized surface layer and the sputtering yield increases to the level of pure Be. The maximum useful temperature of $\mathrm{Be}$ is about $1000^{\circ} \mathrm{K}$, due to evaporation. Although little data exists for oxygen and self sputtering of $\mathrm{Be}$, the results suggest that the yield from these reactions is well less than one.

Although graphite erosion by ion and plasma bombardment has been studied for many years, experiments are still producing new results useful for the understanding of this complicated process. As shown in Fig.1, graphite demonstrates several different types of sputtering behavior during hydrogen bombardment. The sputtering yield of room temperature graphite is virtually independent of incident energy over the range of 20 to $500 \mathrm{eV}$. This is due to the chemical formation of hydrocarbons, which dominates the erosion mechanisms at all temperatures below $700^{\circ} \mathrm{C}$. As the temperature of the graphite is increased above room temperature, the erosion yield increases strongly due to methane formation over the temperature range of 200 to $700^{\circ} \mathrm{C}$. The amount of increase in the yield is dependent on energy, with a factor of two increase occurring for $50 \mathrm{eV}$ ion bombardment and up to a factor of ten increase for $1 \mathrm{keV}$ ion bombardment.

At temperatures above $800^{\circ} \mathrm{C}$, chemical sputtering is suppressed. However, radiation enhanced sublimation, which sputters carbon clusters from the surface independent of the type of incident ion, can occur at higher temperatures. The threshold for radiation enhanced sublimation is in the range 4 of 50 to $\mathrm{i} 00 \mathrm{eV}$, and at low energies the erosion yield of graphite at these 

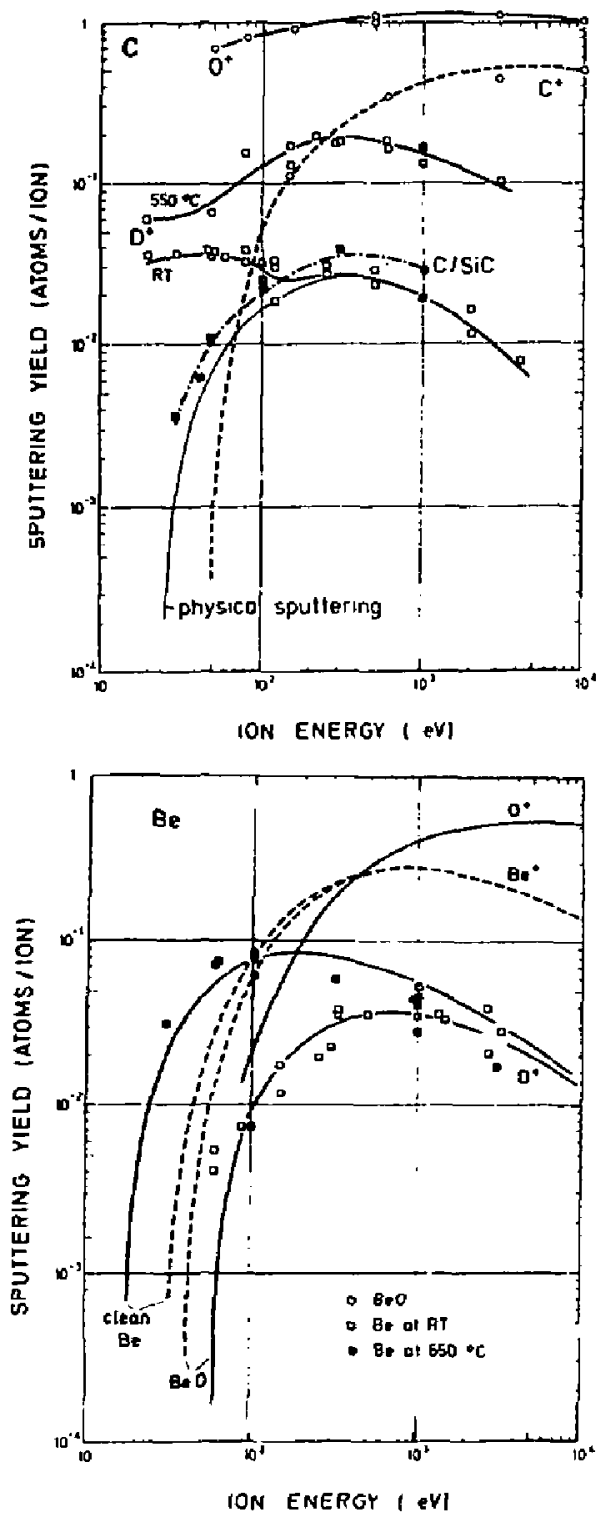

Fig. la Energy dependence of the sputtering yictd of $C$ and $B$ be al rmom temperature with $D, O$, and self ions. Deuterium yields ar elevaled remperatures are also given. De self-spultering data were calculated by TRIM and oxygen sputicring of Bc was estimated from Ne spullering oxidized surfaces. In the case of graphite eputtering with D values for a SiC doped graphice are also given
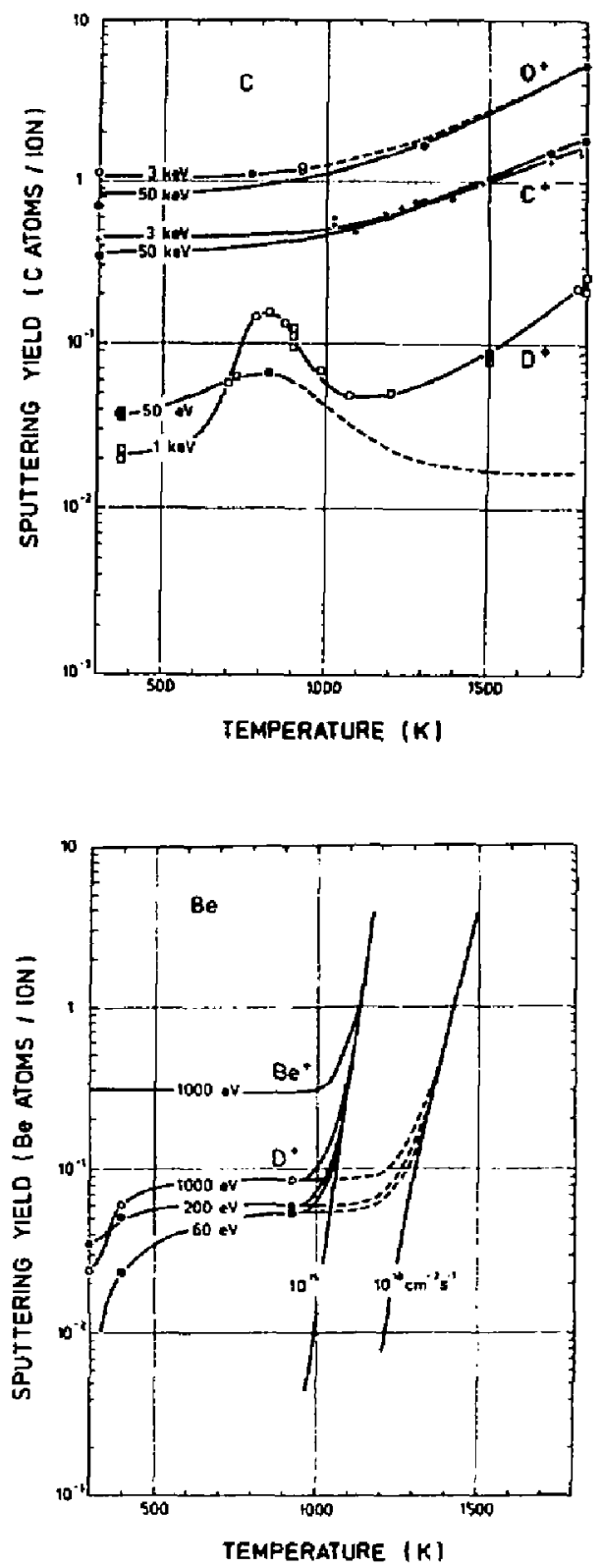

Fig. 16 Temperature dependence ot the sputlering yield of $\mathrm{C}$ and $\mathrm{Be}$ at ditferent energies for $\mathrm{D}, O$ and sellions. For Be the evaporation yield is shown at high temperalures relutive to different inn nuxcs. 
temperatures approaches values anticipated for physical sputtering. There is definitely a hydrogen isolope mass dependence to the sputtering yield of graphite over all the temperature ranges, indicating that the physical and chemical sputtering processes are interrelated. In addition, synergistic effects of atomic hydrogen co-bombardment from Frank-Condon and charge exchange neutrals can lead to enhanced sputtering yields.

Recent experiments in PISCES 5,6 have investigated the erosion of several types of graphites at high incident ion fluxes representative of the edge of tokamaks. Figure 2 shows a direct comparison of the erosion of Poco graphite by $100 \mathrm{eV}$ deuterium ions from an ion beam and from PISCES with a flux difference of three orders of magnitude. ${ }^{7}$ The erosion yields are within a factor of two, indicating that a suggested suppression of the chemical formation of hydrocarbons by high fluxes is not significant. Furthermore, the erosion of several different types of graphite (Poco, ATJ, and Pyrolytic edge plane) in the PISCES experiments show no significant difference in the erosion yield despite very different structures. It has been suggested that the bombardment of graphites by energetic ions universally damages the surface and results in similar sputtering yields. ${ }^{8}$

High fluence hydrogen ion sputtering of graphites leads to a modification of the surface structure. After a fluence of roughly $10^{22} \mathrm{~cm}^{-2} \mathrm{sec}^{-1}$, the surface becomes rough and can even appear honeycombed. In this case, it appears that the weaker bound carbon bulk is removed first by the plasma bombardment, and a structural graphite honeycomb surface remains. The erosion yield of the honeycomb surface is lower than the origirial graphite surface by about a factor of two. This complex surface has been found to getter water in the vacuum system and hydrogen from plasma discharges in PISCES if first activated (cleaned out) by helium or argon discharges. ${ }^{9}$ This behavior is similar to the graphite pumping effect observed on the TFTR and JET inner bumper limiters.

Impurity sputtering of carbon results in very high erosion rates. Figure 1 also shows the erosion yie!ds for oxygen and carbon self sputtering of graphite. In both cases, the erosion yield can approach and even exceed one in some cases, resulting in potentially runaway erosion of the 


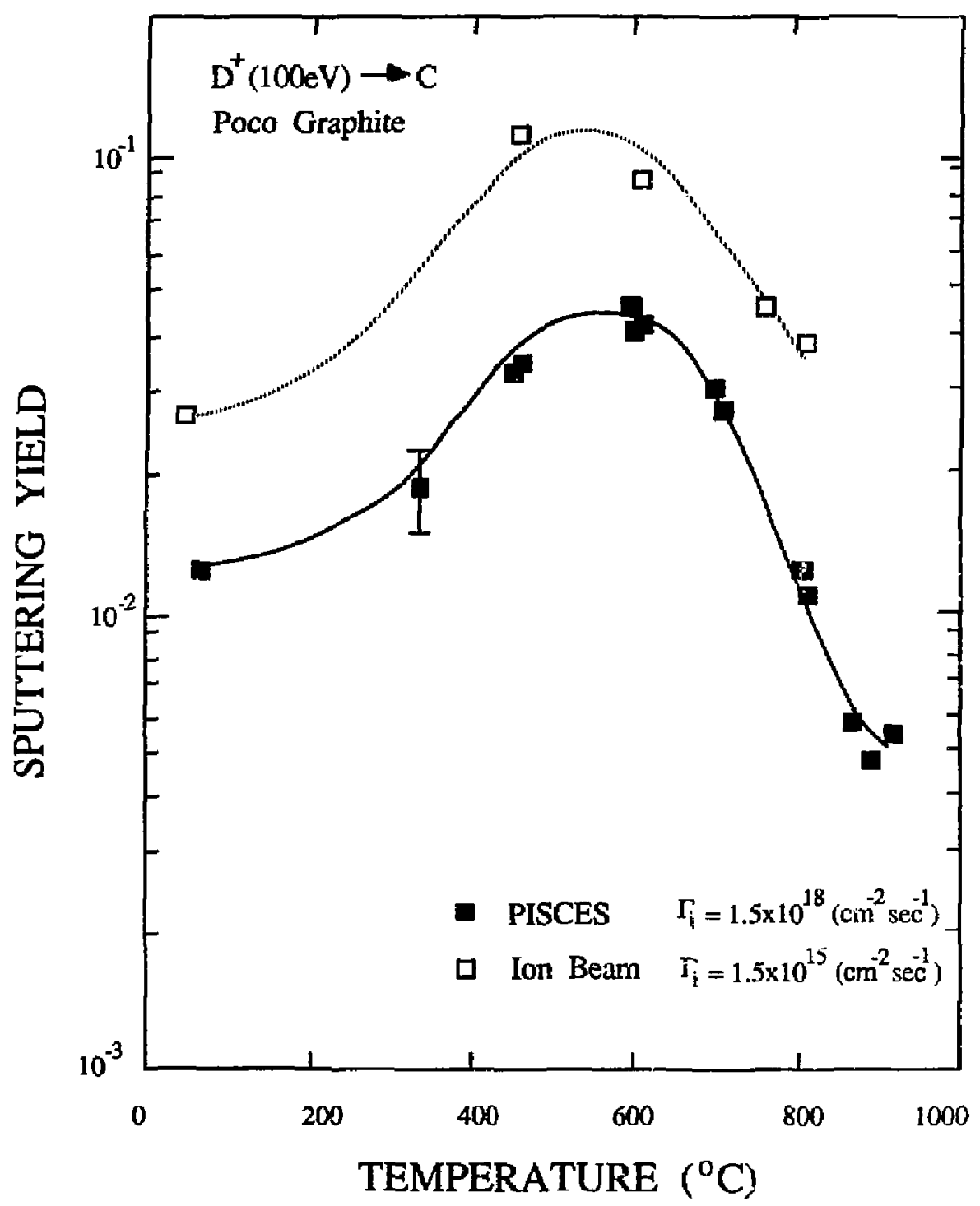

Figure 2. Comparison of the erosion yield of Poco graphite by $100 \mathrm{kV}$ deuterium jons versus temperanure for fluxes of three orders of magnitude difference from an ion beam and from PISCES. 
surface. Chemical sputtering in tokamaks has been carefully investigated in TEXTOR, ${ }^{10}$ where spectroscopic observation of molecular impurity bands are monitored in the plasma boundary, A strong correlation between the oxygen and carbon line radiation reported in these experiments have led to the conclusion that oxygen sputtering with $\mathrm{CO}$ and $\mathrm{CO}_{2}$ formation is one of the dominate sources of carbon impurities in the plasma. Recent results have also indicated ${ }^{l 1}$ that methane formation at the carburized wall is a strong effect, and is partially responsible for the formation of detached plasmas by increasing the low $\mathbf{Z}$ radiating layer in the edge. Suppression of chemical erosion hy metal impurities on the surface has been suggested as one of the reason that chemical effects have not been observed in DITE. 12 This effect was previously found in ion beam experiments on metal contaminated graphite samples. ${ }^{13}$

Carbon-carbon composite weaves have been recently investigated for use in tokamaks due to their good structural properties and improved thermal conductivity. Outgassing studies of C-C composites have shown ${ }^{14}$ that the properties of the materials depend very strongly on the manufacturing process. While some weaves demonstrate outgassing characteristics better than the conventional Poco graphite, others have been found to release large quantities of water and hydrocarbons. The erosion yield of the weaves during ion bombardment also is found to depend on the manufacturing process. The total erosion yield of a ' $4 \mathrm{D}^{\prime} \mathrm{C}-\mathrm{C}$ weave (fjbers oriented in four directions) manufactured by FMI, Inc., as a function of temperature is shown 6 in Fig. 3 with the yjeld for Poco given for comparison. It is found that this material performs as well or better than Poca graphite over the conditions tested. This marerial also was found to have a lower outgassing rate compared to Poco, and has performed well as a limiter in tokamak tests in TEXTOR.

Carefuj examination of the surfaces of $\mathrm{C}-\mathrm{C}$ weaves after exposure to the plasma showed that the bulk and binder in between the fibers and the fibers themselves erode at different rates. This depends on the composition of the binder, and the behavior of the binder is found to also dominate the outgassing of the materials. The fibers, on the other hand, contribute greatly to the thermal conductivity and structural strength of the material. The heat removal capabilities and 


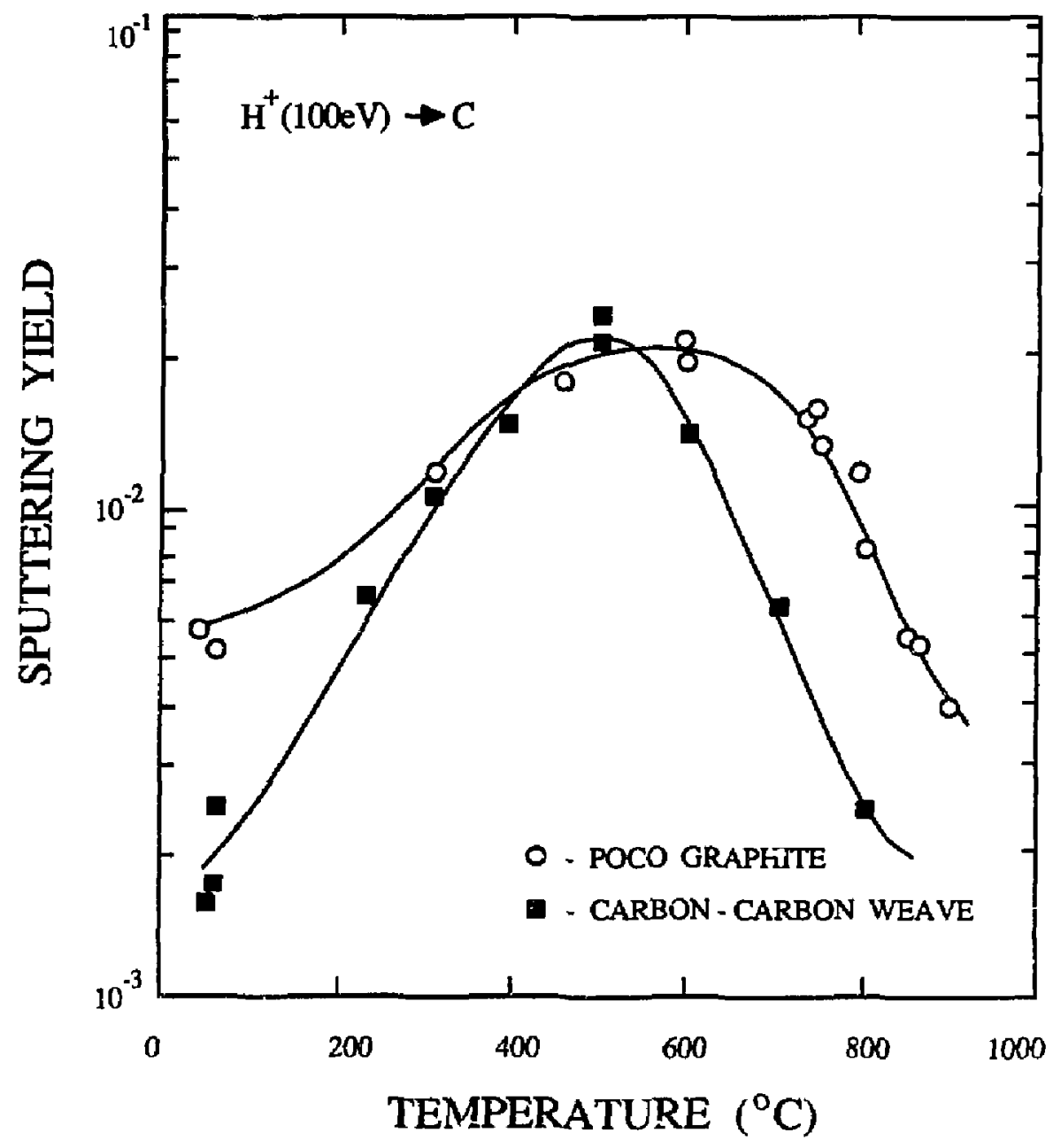

Figure 3. Total erosion yield for $100 \mathrm{eV}$ hydrogen ion bombardment of a 4-D carbon-carbon composite weave materiai versus temperature from reference 6 . Direct comparison of erosion with Poco graphite shows equal or lower levels. 
possibility of withstanding disruption forces have made C-C weaves leading candidates for NET and ETR components. C-C weaves are now being used in TFTR and considered for JET. The performance of these materials during neutron irradiation is discussed in a later section.

Finally, the chemical eiusion problem of graphite has led to the development of carbide coatings intended to suppress hydrocarbon formation. The inclusion of 4 to $5 \%$ of boron and $\mathrm{SiC}$ on the surface of graphite has been found in ion beam experiments to reduce the chemical erosion by a factor of almost ten. These results were achieved for ion energies greater than $1 \mathrm{keV}$, where the hydrocarbon formation rate is a maximum. In experiments at high flux $\left(2 \times 10^{18} \mathrm{~cm}^{-2} \mathrm{sec}^{-1}\right)$ and low energies (100ev), the reduction in the chemical sputtering yield was found ${ }^{6}$ to be less than a factor of 3 . While this is less impressive for applications on limiters and divertor plates, $\mathrm{C} \cdot \mathrm{SiC}$ tiles might still be an effective first wall in ETR. In this case, the predominate bombariment is by charge exchange neutrals with energies in the kilovolt range, where the chemical suppression by the carbide is effective. Other carbides, such as boron carbide, are being pursued to further examine if suppression of chemical sputtering is possible.

\section{2) Redeposition data base}

In this discussion of redeposition, the effects of disruptions and transient events will be left to another section, and only steady state operation will be considered. Deposition of metals on graphite limirers by the plasma has been thoroughly studied over the past few years. 15 The information from these sludies has aided in the undersianding of the transport of materials in the bouldary of tokamaks, and led to successful models which predict the deposition patterns. ${ }^{16}$ In general, it is found that the net erosion of limiters occurs in the regions of high particle energy and flux, and that deposition of metals occurs in regions away from the limiter tangency point deep in the boundary layer. This is understood from the viewpoint that erosion and deposition are competing processes, and that net deposition will naturally occur in regions where the erosion is lower (due to lower energies deeper in the boundary, for example). Complete coverage of the 
graphite surface by over $1000 \AA$ of metals (as recently on the TFTR bumper limiter ${ }^{17}$ ) can occur if the source rate (amount) of metal deposition exceeds the erosion rate.

Redeposition of materials in tokamaks has several consequences. First, there is a mixing of the material exposed to the plasma. Alloys and compounds may be reduced or formed depending on the conditions in the bourdary layer and the PIC surface. The first wall and limiter/divertor surfaces will then be modified by the redeposition and iile plasma-surface interactions. The materials that form are likely to have different properties, which may even depend on the location in the tokamak that they are formed because of different plasma energies and surface temperatures. The results of redeposition in tokamaks are difficult to understand 18 because of the many competiug processes involved and the unknown effects of the startup and ending of the pulsed discharges.

Recently, redeposition experiments have been achieved in PISCES, 5, 19,20 which has the necessary plasma density and electron temperature to ionize sputtered materials and retum them to surfaces exposed to the plasma. In addition, PISCES operates continuously, so that the steady state effects of redeposition can be studied. The results of redeposition experiments are found to depend strongly on the plasma conditions and types of materials tested, as can be expecied from the tokamak results.

From the PISCES results, redeposition is found to always reduce the net erosion rate of surfaces exposed to plasma bombardment. The amount of redeposition is a function of the jonization mean free path (MFY) trom the sample, as shown in Fig.4 where the percentage of the classical sputtering yield is plotted versus MFP. This simply means that particles with a long MFP can escape the plasma near the sample and be deposited elsewhere. The geometry of the surface exposed to the plasma affects the location of the redeposition because of the angular distribution of the sputtered products. Surfaces at a small ang!le with respect to the magnetic field lines (like limiters and angled divertor plates) tend to form regions of net erosion and net deposition due to the transport across and along the magnetic field lines. This behavior is similar 


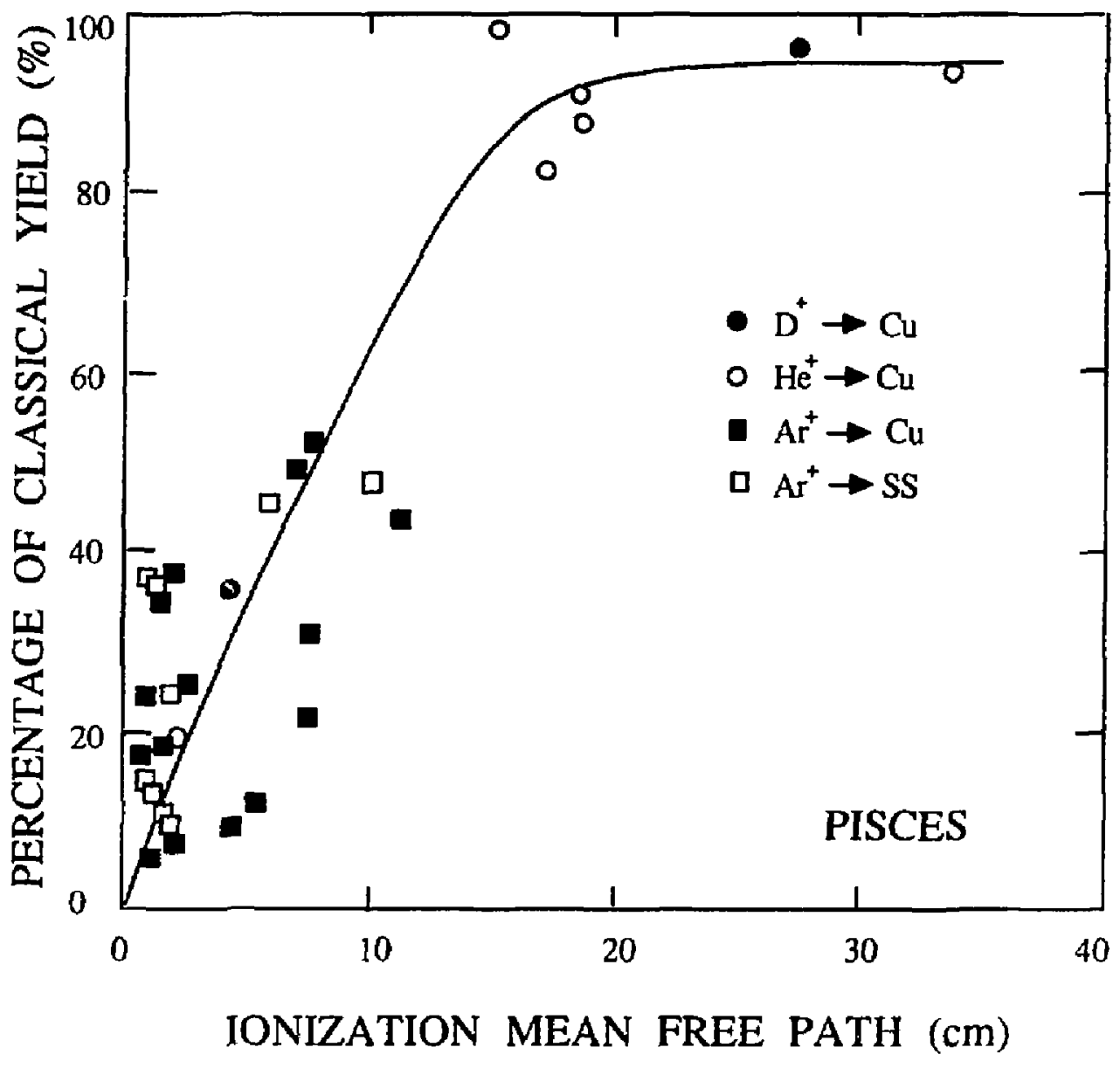

Figure 4. Percentage of standard sputtering yield versus ionization mean-free path in the plasma. As the mean-free path decreases, redeposition teduces the effective sputtering yield. 
to that observed in tokamaks, but exact rates of redeposition depend on the impurity transport in the tokamak edge and cannot be studied in PISCES.

What can be studied in PISCES is the nature of the net eroded and redeposited surfaces. For pure metals, the redeposited material is found to have similar properties as the original metal. The surface structure is essentially the same as that of a heavily sputtered surface. During high density plasma bombardment and strong redeposition, the net erosion yield can be reduced to less than $10 \%$ of the value expected from pure erosion, as seen in Fig.4. The deposition of other materials on the metal surface by the plasma can result in a modification in the surface morphology and sputtering yield. ${ }^{19}$ The effect of the deposition of a small amount of Mo on stainless steel and copper samples was to initiate cone growth above a threshold temperature. The removal of the Mo seed deposition eventually allowed the surface to reform the pure metal surface without the cones. The sputtering yield of the modified surface typically was reduced by a factor of two, probably due to the very porous surface.

The redeposition of graphite is complicated by the fact that the sputiered products have different energies depending on the process that formed them. Chemically formed hydrocarbons tend to leave the surface with an energy corresponding to the surface temperature. These particles are moving relatively slowly, and are quickly ionized and fragmented in the edge plasma of tokamaks- where the plasma densities exceed $10^{12} \mathrm{~cm}^{-3}$ and electron temperatures range from 10 to $10 \mathrm{ReV}$. Physically sputtered carbon atoms leave the surface with several electron volts of energy, and tend to travel long distances compared with the hydrocarbons due to their higher velocities. The deep penetration of sputtered carton helps to explain the large concentration of low $\mathrm{Z}$ impurities in tokamaks with graphite limiters.

Redeposition of hydrocarbons during chemical sputtering was investigated in PISCES 5 It was found that in the temperature range of methane formation $\left(200\right.$ to $\left.700^{\circ} \mathrm{C}\right)$, redeposition of the hydrocarbons on the graphite samples formed a surface dominated by loosely bound soot. Operation in helium at identical plasma conditions did not form the sooty surface. The redeposition did decrease the net erosion of the surface, as shown in Fig.5. As the ionization 




Figure 5. Total crosion yield for $100 \mathrm{eV}$ hydrogen ion bomardment of Poco graphite as a function of temperature in the PISCES facility (from ruference 5). The peak in the curve is due to chemical sputtering. As the hydrocarbon ionization mean-free path is reduced, redeposition causes the net erosion yield to decrease. 
MFP for methane was reduced, the net erosion yield was found to decrease. Recent experiments in PISCES at plasma conditions where physically sputtered carbon was redenosited showed a further decrease in the net erosion yield.

Redeposited layers from the sputering of graphite can have several different forms. In TEXTOR, collection probes positioned near the carburized liner were $\mathbb{k} \ldots$..ld to form an amorphous carbon layer similar to the carburized surface of the liner. ${ }^{11}$ In PISCES, the surface structure of the redeposited layer depends on the temperature of the substrate, and may also depend on the energy of the particles depositing on the surface. Surfaces that appeared amorphous, sooty, and normal graphite-like have been observed for different conditions in the machine.

Unforunately, no laboratory investigations of the redeposition of beryllium have been performed. In the ISX-B21 Be limiter experiments, beryllium was found to spread over the vacuum vessel surface, probably due to redeposition. Heavy wall coverage was observed after melting of the limiter surface occurred. No information on the properties of redeposited Be layers is available.

\section{3) Conclusions for Steady State Operation}

The erosion of surfaces exposed to the plasma is a critical issue for steady state operation. The low $\mathrm{Z}$ materiais presently used in tokamaks have well known erosion rates, even though the erosion process can proceed via several channels during different conditions, as in the case of graphite surfaces. The erosion yields for $\mathrm{C}$ and $\mathrm{Be}$ lie in the range of $10^{-2}$ to almost 1 atoms per ion, depending on the sputtering species and temperature of the material. These relatively high erosion rates will limit the lifetime of plasma interactive components during steady state operation, and may result in impurity accumulation problems in steady state machines.

It has been proposed to reduce this high erosion rate through three processes:

a) selection of better materials with lower sputtering yields

b) redeposition 
c) control of the edge plasma conditions to minimize erosion

Graphite, C-C composite weaves, and Be are still the materials of choice for the next generation of machines and ETR, because better materials have yet to be found. The C-C composites have better materials properties such as thermal shock resistance and higher thermal conductivity, which may affect the choice, but the erosion behavior is somewhat similar to graphite. Present work on advanced ceramics might (hopefully) lead to new breakthroughs for fusion applications.

Redeposition will reduce the net esosion yield of a surface, but the fact that the plasma naturally erodes and redeposits in different locations limits the effectiveness of rede-rosition in extending the lifetime of critical areas. It is possible that limiters and divertors can be designed to enhance the redeposition in critical areas to limiting net erosion, but this is unexplored. The properties of redeposited materials depend on the deposition conditions, and may be controllable in tokamaks.

Control of the plasma edge temperature has long been considered the avenue toward low erosion of PIC. If the edge plasma temperatures are low, then one is below or near threshold and the erosion is reduced. This has been the leading argument from an impurity standpoint for building divertors, where the energy of the ions striking the neutralizer plates can be very low. Unfortunately, one ca, not expect a reduction in the erosion yield for graphite at low incident energies because of the effects of chemical sputtering. The graphite sputtering yield at low temperature is independent of ion energy from 20 to $500 \mathrm{eV}$, and is a relatively high value at 2 to $4 \times 10^{-2}$ atoms per ion for deuterium. This chemical erosion yield is not significantly affected by high ion fluxes. The chemical methane formation peak is decreased at low energy, but never below the room temperature values. The only hope for very low arosion of graphite in tokamaks is to operate at low energy $(<100 \mathrm{eV})$ and high temperatures (above $1000^{\circ} \mathrm{C}$ ). In this case, one is below threshold for radiation enhanced sublimation and at high enough temperature to suppress chemical sputtering. The sputtering yield in this case is below $10^{-2}$ atoms per ion. 
As shown in Fig.1, the sputtering yield for Be is also not significantly reduced by low bombarding energies (data to $50 \mathrm{eV}$ available). Beryllium oxide has a lower yield, but Be limiters above $650^{\circ} \mathrm{C}$ are dominated by the Be erosion yields. No data exists for Be redeposition, but other redeposited metals have been observed to maintain their normal properties.

The argument of low plasma temperatures leading to low erosion in divertors can conceivably be true for the case of moderate or high $\mathrm{Z}$ divertor plates where a true threshold exists. However, experiments in ASDEX and JT-60 with high Z divertor plates have shown significant quanities of the divertor plate material in the core plasma, indicating that the erosion and transport mechanisms are not well understood. Experiments aimed at understanding the impurity production and transport in divertors are needed. High $Z$ limiters have been successfully used in the high density Alcator- $C$ tokamak, perhaps due to low edge temperatures and screening effects by the high density edge plasma.

Controlling the edge plasma temperature to minimize erosion of limiters and divertors exposed to the plasma does not solve the problem of high energy charge exchange neutral bombardment of the first wall. High metal impurity levels in $\mathrm{H}$-mode discharges in divertor machines have been attributed to high energy charge exchange neutral sputtering of the walls. Carbides may be good materials for the first wall to reduce the erosion yield. In steady state, it is unlikely thar carburization of the wall will have an effective lifetime. However, it is possible that other materials deposited on the first wall in the same manner as the carburized layer (boronized amorphous carbon layers or boron carbides, for example) could have significantly improved lifetimes for use in the next generation or steady state machines.

\section{References}

1. H.H.Anderson and H.L.Bay, Sputtering Yield Measurements, in: Sputtering by Particle B.mbardment I, ed. R.Behrisch (Springer-Verlag, Berlin, 1981) p. 145-218.

2. R.A.Langley, J.Bohdansky, W.Eckstein, et.al., Nucl. Fusion Special Issue (1984) 1683.

3. J.Roth, J. Nucl. Mat. 145-147 (1987) 87. 
4. A.A.Haasz, Toronto, private communication.

5. D.M.Goebel, Y.Hirooka, R.W.Conn, et.al., J. Nucl. Mat. 145-147 (1987) 61.

6. D.M.Goebel, J.Bohdansky, R.W.Conn, et.al., submitted to Nucl.Fusion. (1987).

7. J.Bohdansky, R.W.Conn, D.M.Goebel, et.al., Proc. 14th Euro. Conf. on Cont. Fusion and Plasma Physics, Madrid, Spain, June 22-26, Vol 11D.p.794.

8. E. Vietzke, KFA Jülich, private communication.

9. Y.Hirooka, W.K.Leung, R.W.Conn, et.al., submitted to J.Nucl.Mat. (1987).

10. U. Samm, Proc. 14th Euro. Conf, on Cont. Fusion and Plasma Physics, Madrid, Spain,1June 22-26, Vol 11D.

11. C.S.Pitcher, G.M.McCracken, D.H.J.Goodall, et.al., Nucl.Fusion 26 (1986) 1641 ,

12. P. Wienhold, K.F.A. Jülich, private communication.

13. J.Roth, J.Bohdansky, J.B.Roberto, J. Nucl. Mat. 128\&129 (1984) 534.

14. A.E.Pontau and K.L.Wilson, U.S.DOE Fusion Technology Bulletin \#12, Dec. 11, 1986.

15. G.Staudenmaier, J.Vac.Sci.Technol. A3 (1985) 1091.

16. R.T.McGrath, J. Nucl. Mat. 145-147 (1987).

17. M. Ulricksen, PPPL, private communication.

18. D.O.E. Magnetic Fusion Energy Technical Assessment of Critical Issues and Problem Areas in the Plasma Materials Interaction Field, UCLA Report \#PPG-765 (1984).

19. Y.Hirooka, D.M.Goebel, R.W.Conn, et.al., Nucl. Instr. \& Methods-B $\underline{23}$ (1987) 458.

20. Y.Hirooka, D.M.Goebel, R.W.Conn, et.al., J. Nucl. Mat. 141-143 (1987) 193.

21. P.K. Mioduszewski, et.al. ORNL Report (1986). 


\section{Tritium Issues for Steady State Fusion Reactors}

In the area of Plasma Materials Interactions (PMI), the principal tritium issue for steady-state reactors of the future is permeation. Tritium inventory also remains an issue, but of less concern than for present day machines. When TFTR and JET begin tritium operations late in this decade, especially during start-up when tritium availability issues arise. These constraints are brought on by the lack of cleanup abilities, environmental considerations, or simply self-imposed limits. Because these reactors do not have breeding blankets and do not require processing of tritium, the total inventory is strongly dependent on the retention in the plasma-interactive components. Steady-state reactors will not have these severe limits on tritium inventory, and much of the total inventory will be tied up in the processing facilities; thus, while inventory in the first wall and structural materials will remain an issue for safety and environmental consideration, permeation will move to the forefront. For these advanced machines, tritium permeation into the coolant and possible releases to the environment pose the mr st serious problems.

\section{1) Tritium Inventory}

As stated above, the inventory of tritium will not be much of a driving force for steady-state reactors that it has been for present-day machines. When kilogram quantities of the ritium will be tied up in the blanket and processing facilities, the need to strictly control the amount of tritium in the plasma-facing materials is somewhat lowered in importance. There are two processes that could significantly increase the inventory in the first wall to the point that it would be of major concem. The first of these is the trapping of tritium in defects produced by neutron damage. The database available for the effect of radiation damage on the retention of hydrogen isotopes in materials is very limited, The second process that could affect the inventory is the co-deposition of tritium and other wall materials. Recent studies ${ }^{1}$ have shown that the co-deposition of carbon and hydrogen isotopes are very important in determining the recycling of the fuel for today's 
fusion reactors. It is difficult to predict the effect this process could have on the tritium inventory for steady-state machines where continuous wall erosion with re-deposition will occur.

\section{2) Tritium Perneation}

Tritium permeation through first walls, limiters, or divertors subjected to energetic tritium charge exchange neutral bombardment is a potentially serious problem for steady-state fusion reactors operating at elevated temperatures. High concentrations of tritium in the near-surface region can be achieved by implantation of the charge exchange neutral flux combined with a relatively slow recombination of these atoms into molecules at the plasma/wall interface. Because of this large concentration of mobile tritium near the inner wall surface, a concentration gradient is established, causing tritium to diffuse into the bulk and eventually to the outer wall surface where it can enter the first wall coolant. The cost required to remove this tritium can be excessive. An excellent example of the possible permeation rates and costs is that for the onceproposed direct convertor for the MARS reactor. ${ }^{2}$ This plasma interactive component located in the two end cells of the tandem mirror fusion reactor was predicted to receive a high flux of energetic tritons. The original design called for a water-cooled vanâdium structure. Vanadium offers many attractive features, including exceltent thermal conductivily, high melting point, and low neutron cross-section. Unfortunately, vanadium also has a strong (exothermic) affinity for hydrogen. Calculations of the steady-state tritium permeation predicted valies in excess of $1 \mathrm{x}$ $10^{8}$ curies/day. This rate would raise serious safety and environmental ccncerns, and the necessary equipment required to remove the tritium from the coolant was estimated to cost in excess of ten billion dollars.

The use of permeation barriers is one possibility for solving many of the tritium release problems associated with steady-state reactors. An example of the effectiveness of this technique is demonstrated by the vanadium problem listed above. The simple lining of the coolant side of the vanadium with a $0.1 \mathrm{~mm}$ copper layer was predicted by computer codes to reduce the release from $1 \times 10^{8}$ curies/day down to a much more reasonable 300 curies/day ${ }^{3}$. This also reduced the 
capital cost of the tritium recovery system from ten billion dollars down to one million dollars. Although barriers offer large returns in the form of much lower predicted releases, realistic laboratory and device tests will be required prior to their use. It is very difficult to find a barrier material that retains its integrity under the conditions that will exist in a steady-state fusion reacior.

A reasonable database exists for fundamental hydrogen properties such as diffusion, solubility, and trapping in structural, metals like stainless steel, molybdenum, vanadium, etc., but not for most low atomic number materials. The molecular recombination at the surface has been measured for only a few selected metals, and only very limited data is available for the heat of transport, important parameter for diffusion in a thermal gradient. Dynamic interactions between hydrogen isotopes and neutron-produced radiation are poorly understood. The database for nonmetals is almost nonexistent.

\section{3) Laboratory Experiments}

The Tritiun I'lasma Experiment ${ }^{4}$ (TPX) located at Sandia National Laboratories in Livermore, California, and PISCES 5 located at the University of Califomia in Los Angeles are the two principal experimental devices in the United States performing measurements vital to the tritiurr: transport database. The TPX facility at the Tritium Research Laboratory of SNLL is the only operating tritium plasma apparatus devoted to plasma-materials interaction research. Magnetic confinement is used to contain the plasma, and heating is provided by a pair of 200 watt $\mathrm{rf}$ generators operating in the electron-cyclotron regime at $400 \mathrm{MHz}$. Ion densities on the order of $10^{11}$ ions $/ \mathrm{cm}^{3}$ are attainable with average ion energies of $7 \mathrm{eV}$. By applying a negative bias to the experimental samples, particle energies between 15 and $300 \mathrm{eV}$ are possible with tritium fluxes on the order of $1 \times 10^{17}$ ions $/ \mathrm{cm}^{2}-\mathrm{s}$.

Because TPX operates with tritium, many experiments can be performed that would be impossible with hydrogen or deuterium. Tritium, being a radioactive isotope, is easily detectable at very low concentrations. TPX also has extensive diagnostic equipment available. Two 
quadrupole mass analyzers allow measurements of the gasses released from the front and back of permeation samples. An in-situ Auger electron analyzer permits the examination of the surface impurities during permeation experiments.

PISCES is a continuous' 5 operating plasma device at UCLA which achieves hydrogen plasma densities of $10^{11}$ to $10^{13}$ ions $/ \mathrm{cm}^{3}$ and electron temperatures of 5-25 eV. Particle fluxes of $2 \times 10^{19}$ jons $/ \mathrm{cm}^{2}$-s are obtained on negatively biased samples. Target fluxes in excess of 1 $A / \mathrm{cm}^{2}$ are produced over an area of $50-80 \mathrm{~cm}^{2}$ One of the most unique capabilities of PISCES is to measure redeposition effects.

There are many facjlities other than TPX and PISCES performing studies on tritium issues. Many laboratories are using accelerators to implant hydrogen isotopes and subsequently . determine their profile with nuclear reaction analysis. There are also facilities performing basic permeation studies where the effects of surface impurities, trapping, temperature gradients, and others on the permeation are being examined.

\section{References}

1. W.L. Hsu and R.A. Causey, Procedings of the 23rd Nat. Symp. Am. Vac. Soc., Oct. 1986, Baltimore, MD.

2. M.I. Baskes, A.E. Pontau, K.L. Wilson, and W.L. Barr, J. Nucl. Mater. 122 and 123 , (1984) 1511.

3. K.L. Wilson, ASM Conference on Materials for Future Energy Systems, May 1984, Washington, D.C.

4. R.A. Kers*, J. Vac. Sci. Technol. 20 (1982) 1267.

5. D.M. Goebel, G.A. Campbell, and R.W. Conn, J. Nucl. Mater. 121 (1984) 277. 


\section{Transient Behavior}

Disruptions, which are observed in all current tokamaks, are characterized by a rapid reduction in the plasma current accompanied by the localized deposition of much of the stored plasma energy on the tokamak inner surface. The forces produced during the current decay phase of disnuptions determine to a large extent the structural support required for the vacuum vessel and for plasma interactive components (PIC). The major forces generated during this phase resuit from coupling of the induced eddy currents in the vessel and other PIC with the externally applied magnetic fields.

There are uncertainties in the magnitude of the induced forces and of the transient heat fluxes. Disruptions are observed to have two phases, a very rapid quench of a majority of the plasma energy in times of much less than a miliisecond, and then the release of the energy associated with the plasma current decay. Current decay times of typically one mega-ampere per millisecond have been observed in TFTR and JET. Since all of the stored energy in the tokamak is released in a time of milliseconds, the heat fluxes can be extremely high and result in vaporization and melt layer formation on the PIC.

Steady state devices must have plasma interactive components designed to withstand the forces and heat fluxes associated with disruptions. Since the critical issues in steady state operation of PIC are continuous heat removal and high fluence erosion, the added complication of building components to handle disruptions can modify the PIC design and severely limit performance. In fact, the requirements for dissipating the disruption energy are often the opposite of the requirements for optimum continuous heat removal. For example, thick graphite tiles which provide a large surface area for sublimation during disruptions are used in present machines. In general, thin material layers between the plasma and cooling system are desired for the high heat removal and low stress cooling systems. High thermal conductivity materials like copper tend to be good elecrical conductors, and are therefore subject to large forces during distuptions. Fisally, Be forms melt layers and droplets during high heat flux bombardment and 
disruptions, which is dangerous for any PIC cooling system. The problem of disruptions may eliminate the use of $\mathrm{Be}$ in steady state devices solely for this reason. It is clear that taking into account disruptions in the PIC designs can seriously affect the performance of components for steady state operation in the tokamak.

Ultimately, methods for controlling and eliminating disruptions will be needed for steady state machines. The best hope for eliminating the disruption problem is the detection of disruption precursors followed by the activation of suppression or soft landing feedback systems. Obviously, research efforts aimed at the design of durable PIC must continue for steady state machines. If PIC capable of surviving multiple disruptions cannot be designed, future tokamaks may require frequent component replacement. This will seriously affect the availability of the device. 


\section{Steady State Heating and Fueling Components Issues}

In examining the question of the issues of steady state heating and fueling components, there is a natural division between those portions of a particular system which are extemal to first wall, blanket, and shield portions of an ETR and those which are exposed to plasma heat and particle fluxes and/or significant neutron fluxes. Those subsystems in the first category can in general be developed and tested independently of the plasma facility. In the second category are ICRH and LH launchers. In the discussion of steady state issues, we propose to focus on those associated with systems which fall in this second category. In some sense this is a subset of the issues posed by in-vessel components as a whole, but modified by additional engineering requirements. We will not discuss those problems which are common to all in-vessel components, such as lifetime, heat removal, coolant compatibility, and erosion, but will only call out those features which add to or modify the set of common issues.

\section{1) Hearing Systems}

a) Neutral Beams. There are no sensitive components within the device envelope. However, if for any reason, such as high gas pressute, there is significant reionization, then high heat fluxes in the beam duct may result.. Because a rather large aperture is needed to provide access for the beams to the plasma, neutrons streaming into the beam transport components may be sufficient to degrade insulator performance, cause significant heating of cryogenic pumps, and activate components which have high maintenance demands. This impacts the lifetime and maintenance schedule during steady state operation. Neutral beam lines with special geometries to reduce the streaming of neutrons into sensitive areas such as the ion source and accelerator have been designed and are under development at this time. The degree to which the above are a concem for ETR depends on design details and must be assessed in the context of a particular 
facility. Steady state neutral beam development has its own set of issues, but are separate from this discussion.

b) ECRH. It is not likely that a vacuum window that directly faces the plasma is required for ECRH systems. However, neutron fluxes which impact window lifetimes may be present even with waveguide bends due to reflection; and this must be assessed for each design. Mirrors and/or mode convertors may be required inside the vacuum vessel for quasi-optical launching systems, and the engineering problems of these components will depend on the source power density. The power density of conventional microwave sources should not overly complicare the design of in-vessel components, but the much higher power density of free electron lasers may lead to significant heat loads and require special attention. In addition to the usual problems of plasma facing components, the effects of high power density microwaves may be a problem if a free electron laser source is utilized.

c) ICRH. Antenna launchers for ICRH in steady state machines face all of the issues presently known for pulsed systems; i.e. impurity production from the Faraday shield, arcing and voltage stand off in the antenna structure, heat transfer, and erosion. The fact that the antenna must be very close to the plasma means that it faces the same issues in steady state devices as limiters. In fact, impurity generation and accompanying enhanced erosion rates due to local ion heating are likely to exacerbate the types of problems and issues presently associated with limiter and divertor plates. Waveguide launchers can have less direct exposure to the plasma edge, which reduces somewhat the issues just mentioned. However, problems with breakdown is the waveguide due to photon and neutron irradiation of the surfaces will exist in continuous operation, and must be assessed.

Active tuning of the if system to get optimum coupling and power transfer to the plasma will be an issue during startup and steady state operation of ETR. This requires an assessment of the capabilities of variable capacitors and stubs for active control of the tuning, which depends on the 
specific design of the system. The if system performance will add electrical conductivity as a figure of merit for some pars of the launcher. This will impact the materials selection and lifetime. There may also be a need for insulators in the system which may be exposed to some fraction of the first wall flux. These insulators will degrade in performance during steady state exposure to neutrons and plasma flux.

d) $\underline{\mathrm{LH}}$. Except for insulators, all of the issues discussed on ICRH will be present.

\section{2) Fueling Systems}

a) Gas Fueling. Fueling of the plasma by gas puff, if applicable for ETR, is a simple and well known technology. There are no special steady state issues with gas fueling systems. Dut to the ability to place the inlet valve and feed system very far from the vessel, activation is not considered a problem. The time response of the total gas feed system must still be adequate to control the plasma density.

b) Pellet Iniection. Pellet injectors, while requiring line of sight access to the plasma, can be located several meters removed from the vessel and require small apertures. This strongly reduces the neutron shielding requirements. However, as with all the auxiliary heating and fueling systems, activation of the components will be an issue which effects lifetime and maintenance. Tritium inventory will be an issue for ETR. There are also issues associated with the development of steady state pellet injectors, but this will be discussed separately. 


\section{Diagnostics and Plasma Control}

Diagnostics for long-pulse tokamaks have been discussed to a limited extent in the INTOR studies, ${ }^{1-3}$ with each phase concentrating on a different aspect of the diagnostic requirements. This work was done before TFTR or JET results were available so that diagnostic experience on these large, high temperature plasmas was not availabie to the study groups. Experience and new diagnostic developments on these machines are now available and a review of the INTOR recommendations is in order. The INTOR study is not sufficient to completely satisfy the requirements for diagnostics on a steady-state device, however. While the reliability, radiation hardness, and types and uses of the diagnosties have been addressed, INTOR is still a pulsed machine (100s pulse, $35 \mathrm{~s}$ recharge time). There are several standard tokamak diagnostics which require pulsed operation and do not work in true steady-state. Standard magnetic loops and bolometers are obvious examples.

This report gives a short review of the structure and purpose of a diagnostic system based on the INTOR studies and then concentrates on those specific aspects of the diagnostic system which are unique to steady-state devices and not a simple extrapolation of existing diagnostics. It seems that the area of diagnostic coverage which requires the most attention is for those diagnostics which measure parameters required for gross plasma control and optimization of the power production. The measurement of these parameters is discussed and suggestions made for improvement or further development.

\section{1) Diagnostic system overview}

The diagnostic systems on a steady-state device must fulfill several functions which have various degrees of reliability, performance, and availability. A general overview of diagnostic functions is the following: 

a) Normal plasma start-up and shut-down.
b) Control of gross plasma parameters: plasma current, density, position and shape.
c) Optimization of power prafuction.
d) Detection of disruption precursors.
e) Plant function: coil currents, temperatures, power distribution, etc.
f) Safety system and interlocks.
g) Calibration
h) Physics and engineering studies.

As stated, these functions generally use different and independent diagnostic systems. However, there are strong interactions between the various systems. For example, power supply problems will trigger the plasma shut-down system. The following is a short description of the purpose of each of these diagnostics systems in a steady-state device.

a) Plasma startup and shut-down. This aspect of device operation is presumably well understood as it is common to pulsed and steady-state machines. It would be natural to use the same diagnostic systems for startup and shut-down as are used for steady-state operation provided sensitivity and dynamic range are not a problem. Startup and shut-down are relatively rare but significant occurrences in a steady-state device, and as such, require very reliable diagnostics. The optimum solution to the reliability problems is to use devices which are will calibrated and known to be in good working conditions, i.e., the same devices used to run the machine.

b) Control of gross plasma parameters. This aspect of steady-state diagnostics requires some discussion and will be addressed in a later section of this report. The main problem in this area is that plasma magnetic measurements as implemented on present-day devices cannot be extrapolated to steady-state machines. Magnetic loops are used to measure various components 
of the magnetic field produced by the plasma and poloidal field coils. The time varying voltage induced in the sensor coil is integrated to deduce the magnetic flux. The integration methods work well only for a finite period of time, ranging from secends to minutes, depending on the sophistication and quality of the electronic circuits doing the integration. Steady-state control will require new techniques.

c) Optimization of power production. At present, this area of diagnostic development is pure speculation. It is not apparent at this time just how the optimum conditions of ion and electron temperature, tritium and deuterium density, helium ash removal, impurity production, and plasma fueling are to be measured and controlled. What is clear is that no diagnostic with a single measurement and control function is available to optimize power production. More than likely, the output of several different diagnostic devices will have to be combined to control power output in a predictable fashion. Since this area is so poorly defined, only general statements can be made with respect to steady-state devices. It is assumed that some minor extension of the diagnostic instruments needed to run the machine can be devised to optimize and control power production.

d) Detection of disruption precursors. A dedicated system of diagnostics is required to detect precursors which lead to a disruption. At present, there is no agreed upon set of signals which reliably predict the onset of disruption. Presumably, some combination of magnetic fluctuation, $X$-Ray wave, density, radiated power, and $Z_{\text {eff }}$ measurements will result in a consistent indicator of imminent disruption. If non-rotating, locked MHD modes prove to be an effective indicator of disruption, ${ }^{4}$ then magnetic sensors with d.c. capability will be required.

e,f) Plant function, safety system andinterlocks. Diagnostics in these categories are straightforward extensions of present day systems since there is little or no difference between pulsed 
and steady-state devices with respect to these areas. The critical issues are reliability, availability, and maintenance which have been recognized in the INTOR study as needing critical assessment.

g) Calibration. Any measurement system requires periodic calibration to verify that it is in working order. The most reliable calibration is one which is direct in nature, i.e., simulates the pnysical parameter which is to be measured when the diagnostic is in use. In pulsed devices or ones with low availability, these calibration procedures can be done during maintenance periods or even between pulses. Calibration in steady-state devices will either have to be done infrequently, by more indirect means, or by other diagnostic devices on the machine which are not necessarily suitaible for steady-state use but are accurate for calibration purposes. It may also be necessary to purposely perturb the device, for instance, change the fueling rate, in order to verify and calibrate the various relationships and constants used in the device control circuitry and control algorithms as long term drifts may cause these "constants" to change. This type of calibration is also needed on pulsed devices, but is not usually done while the machine is in the run mode.

h) Physics and engineering studies. There will certainly be diagnostics on a steady-state device to monitor the long term condition of the first wall, blanket, and stressed components. These diagnostics will have to be highly reliable or remotely maintainable to insure that a complete history of these critical machine components is obtained.

Table I presents a list of the physical parameters which will probably require measurement, possible diagnostic devices to provide the data, and which aspects of the diagnostic system will use the data. The use catagories are with respect to the different diagnostic systems just discussed. 
Table I. List of Measurements, Diagnostics and Uses for Plasma and In-vessel Parameters

Parameter

Diagnostic

Use Catagory

Plasma Current

$I_{P}$

$J(r)$

Rogowski coil

$a, b, d$

Multi-channel Faraday rotation

using $\mathrm{CH}_{3} \mathrm{OH}$ laser

$a, c, d, f$

Polarized He line emission 5

(requires He diagnostic neutral beam) g, $\mathrm{h}$

Thomson scattering

( $\omega_{c e}$ modulation, requires fast optics)

g,h

Polarized Li emission

(requires Li diagnostic neutral beam) $\quad \mathrm{g}, \mathrm{h}$

$\mathbf{I}_{\mathrm{i}, \text { plasma inductance } \quad \text { Magnetic loops }}$

(requires elongated plasma) a,b,d

Loop voltage

$\mathrm{V}_{\mathrm{L}}$

Magnetic loop

$a, b, d$

Plasma position

$\mathrm{R}$ (major radius)

Magnetic loop, flux coils

$a, b$

a (minor radius)

$\mathrm{R}$ plus limiter position

$a, b$

Soft X-ray intensity profile

$a, b$

$D_{\alpha}$ emission array

$a, b$

$\mathrm{z}$ (vertical position)

Magnetic loops, flux coils

$a, b$

Plasma Shape

Elongation/triangularity

Magnetic loops, flux coits

$a, b$

Soft X-ray intensity profile

$a, b$

$D_{\alpha}$ emission array

$\mathrm{a}, \mathrm{b}$

Shafronov shift $(\Lambda)$

Magnetic loops, flux coils

$a, b$

Stored Energy

$\beta_{p}$

Derive from $\Lambda$ in elongated plasmas or

calculate from $\Lambda$ and $\mathrm{J}(\mathrm{r})$

$\mathrm{c}, \mathrm{d}$

$\beta_{\text {perp }}$

Diamagnetic loop

c,d 
Electron density

$n_{e}(r)$

Line average $n_{\mathrm{e}}$

Electron Temperature

$T_{e}(r)$

Certral $\mathrm{T}_{\mathrm{e}}$

Non-thermal energy

Ion Species Temperature

$T_{i}(r)$

Avcrage $T_{i}$

Ion Species Density

$\mathbf{n}_{\mathbf{i}}(\mathbf{r})$

Average $T_{\mathbf{i}}$

Impurities

$Z_{\text {eff }}(r)$
$\mathbf{n}_{Z<20}$

Thomson scattering g

Reflectometer

b,c

Multi-channel interferometer

(Microwave, $\mathrm{CH}_{3} \mathrm{OH}, \mathrm{CO}_{2}$ ) b,c

Microwave, $\mathrm{CH}_{3} \mathrm{OH}, \mathrm{CO}_{2}$ interferometer $\quad \mathrm{a}, \mathrm{b}, \mathrm{c}, \mathrm{d}$

Thomson scattering g

$\omega_{c e}$ cyclotron emission c

$X$-ray pulse-height-analysis c

Hard X-ray detector a,d

Charge exchange recombination spectroscopy

(require diagnostic neutral beam) $\quad c, g$

X-ray crystal spectrometer c

Collimated neutron flux c

Neutron spectrometer c

Mass selective charge exchange

(require diagnostic neutral bean) c

UV spectroscopy c,g

Neutron flux c

Mass selective charge exchange

(require diagnostic neutral beam) c,g

Flouresence spectroscopy g

High energy charged fusion products $\quad b, c$

Neutron spectroscopy

(measure DD and DT production rates) c

$\mathrm{D}_{\alpha} \mathrm{T}_{\alpha}$, He line emission combined

with fueling and exhaust rates c

Residual gas analyzer b,c

Visible brehmstrahlung c,d

$X$-ray pulse-height-analysis $\quad c, d$

UV spectroscopy g

Visible spectrosccpy g 
$\mathrm{n}_{2>20}$

MHD Activity

Internal coherent modes

Sawteeth

Near surface and incoherent modes

Locked modes

Energy Loss

From main plasma

Power flux to the wall
$\mathrm{X}$-ray puise-height-analysis

$\mathrm{X}$-ray crystal spectrometer

$$
c, d, g
$$

X-ray wave detector d

Mimov coils d

Soft $X$-ray channel $\quad c, d$

$\omega_{\text {ce based }} T_{e}$ điagnsolic $\quad c, d$

Mimov coils d

Flux loops d

Bolometers $\quad c, d$

Charged particle detectors $c$

Neutral particle detectors c

Wallhimiter temperatures

(thermocouples, IR pyrometers $\quad b, c, f$

Power monitors in sub-system

coolant systems

$b, c, e$

First Wall Integrity

Radiation aamage

Surface analysis system

Sample probes

e

Surface analysis system

Sample probes

e

Plasma TV

Clearly, the list of diagnostics presented in Table I cannot all be installed on a steady-state device. One of the areas which must be addressed is the selection adiagnostics which minimizes complexity, maximizes reliability and availability, and fulfills the function of a complete diagnostic system. 
2) Specific diagnostic problems unique lo steady-stare

a) Mauneric loops. As presently implemented, all magneric loops (Rogowski, diamagnetic, flux, and saddle coil) use electronic circuits to integrate the time varying incuced voltage to infer the magnetic flux through the coil. Such schemes sie inherently pulsed in nature as the devices must be occasionally reset: even a small offser, when integrated long enough, will lead to unacceptably large errors in the inferred flux. There are suggested solutions of this problem:

i) Develop super-conducting loops and read-outs. These devices measure the total magnetic flux crossing the loop but do rot suffer the inherent time constraints of conventional loops as the current does not de:ay. So as not to perturb the plasma, these lo jps would have to be sinall. For a diamagnetic loop, the superconductiig loroidal field coils themselves could be ased as a was done on PilX.s

ii) Develop magnetic sensors based on magnrtc-optic effects using fiber optics as sensors and read-outs. These devices would be less stinsitive to radiation damage than conventional solid state sensors. Possibilities are the following:

1) Magneto-mechanical effects such as magneto-striction measured by a fiber-optic based interferometer.

2) Use magneto-optic effects in the fibers themselves such as Faraday rotation or the Cotton-Mouton effect.

Most likely, a combination of cuperconducting and fiber optic based magnetic sensors will have to be used.

b) Bolometers. Most bolometers used on tokamaks today derive a power flux to a thermally isolated surface by measuring the temperature rise of the sensor and either electronically or computationally taking the time derivative of the temperature to infer a power tlux. In a steadystate device, a bolometer of this type would eventually come into thermel eyuilibrium with its 
surroundings resulting in zero net power flux to the sensor. Transient-type bolometers rely on the heat capacity of the sensor as the calibration standard of the instrument.

A steady-state device would use the themal conductivity of a sensor element lo determine the quasi-steady-state heat flux from the plasma. Conceivably, a steady-state bolometer would have a cooled reference plane which would cool the sensor element. The temperature difference between the front and back surfaces of the sensor element would then be proportional to the power flowing through the device using the relationship

$$
P=k\left(T_{\text {front }}-T_{\text {back }}\right) / 1
$$

where $P$ is the absorbed power $\left(W / \mathrm{cm}^{2}\right), k$ the thermal conductivity $(W / c m-K), T\left({ }^{\circ} K\right)$ the temperature of the front or back surface, and $\mathrm{t}(\mathrm{cm})$ the thickness of the sensor element. The time response of such a bolometer would probably be rather slow, so that a combination of a steadystate and transient bolometer is required to adequately measure the radiated power flux on all time scales.

c) Plasma density measurement. Virtually all interferometeric-type plasma density measurements suffer from a major problem in steady-state, high availability machines. Namely, either in-vessel mirrors or vertical through-vessel lines-of-sight are required. Radial line densities, if they are not tangential to the plasma column, need a reflector on the inner wall of the device. It has not been demonstrated at this time that a mirror will maintain its properties after long exposure to the in-vessel environment. The $1 \mathrm{~mm}$ micro-wave interferometer carbon in TFTR has not shown signs of degradation to date, however, operational experience on a low availability machine like TFTR is difficult to apply to a steady-state device with confidence. Vertically viewing cords suffer from the necessity of having windows on the bottom on the machine where dirt and debris are likely to accumulate.

An attractive solution to this dilemma is the use of a density reflectometer such as that being developed for JET, 7,8 This kind of atsity diagnostic uses micro-waves at different frequencies 
launched into the plasma to determine density profile. Each frequency is reflected from the plasma at the density corresponding to the cut-off density for that frequency; hence, each frequency determines a particular density. The position of that censity is determined by the phase delay of the wave. This system does not require internal mimors or vertical sight lines, is robust and yields a density profile on a rapid cime scait.

Thomson scattering measurements of the electron density and temperature are valuable as a calibration standard for other, less direct methods of determining these two plasma parameters. Unfortunately, the standard system in use today has a large, delicate optical system near the plasma in order to give good spatial resolution. Recently on JET, a new type of Thomson scattering system has been developed, known as Thomson scattering LIDAR.9,10 In this system, time-of-flight of the back-scattered laser light is used to provide adequate spatial resolution for electron density and temperature. Near machine optics are eliminated and little accuracy is sacrificed. Such a system seems to be attractive in a steady-state device since the windows and optics could be in relatively protected areas, resulting in a fairly robust, easy-toservice diagnostic. 


\section{3) Recommendations}

The general guidelines stated in the INTOR reports, that diagnostic devices must be radiation hardened and of high reliability, are critical. Remote maintenance is a necessity for cievices inside the shield area and this requirement has a profound effect on the design of the apparatus.

Specifically:

a) Diagnostics should be developed which require no delicate in-vessel hardware, especially if this hardware is not accessible from the outer midplane of the machine. If hardware must be in the near-vessel environment, then it should be robust, i.e. waveguide rather than windows.

b) Diagnostic views should be from above or at the midplane of the machine. Bottom views are not desirable as this area collects debris.

c) Special attention should be given to magnetic and bolometric-iype diagnostics in order to develop steady-state instruments.

d) Calibration is a critical issue in steady-state as the availability of the diagnostic instruments for calibration and maintenance is severely limited in a steady-state situation.

e) The kinds and number of diagnostic devices must be carefully studied in order to determine a set which is reliable, maintainable, and sufficient to run a steady-state machine.

f) Finally, the incorporation of the diagnostic instruments must be taken into account in the initial design of the device. Attempting to add on to an essentially finished design will compromise the requirements of high reliability, availability, and maintainability. 


\section{References}

1. International Tokamak Reactor, Zero Phase., Report of the Intemational Tokamak Reactor Workshop by the IAEA and held in Vienna, 1979, IAEA Vienna, 1980.

2. International Tokamak Reactor, Phase One., Report of the International Tokamak Reactor Workshop by the IAEA and held in Vienna, 1980 to 1981, IAEA Vienna, 1982.

3. International Tokamak Reactor, Phase Two., Report of the Intemational Tokamak Reactor Workshop by the IAEA and held in Vienna, 1981 to 1983, IAEA Vienna, 1983.

4. Workshop on the Physics Operation of Tokamaks, held at the Princeton Plasma rhysics Laboratory, Sept. 16-18, 1987, M.G. Bell chairman.

5. F.C. Jobes, "A Proposed Poloidal Field Mensurement Scheme", BAPS 32 (1987) 1928.

6. P. Thomas, "Diamagnetic Flux Measurement Using the PDX TF Coils", Princeton Plasma Physics Report PPPL-1979, Feb. 1983.

7. C.A.J. Hugenholtz and A.J. Putter, "Technical Description of the Detection Systems and Data Acquisition for the JET Multichannel Reflectometer", Associate Euratom-FOM, Institut voor Plasmafysica, Rijnhuizn, the Netherlands, Rijnhuizn Report 86-170.

8. A.E. Hubbard, et.al., "A Simple Fixed-Frequency Reflectometer for Plasma Density Measurements on JET ${ }^{*}$, J.Phys. E: Sci Instrum. 20 (1987) 423.

9. H. Salzmian and K. Hirsch, "Proposal for a Time-of-Flight Thomson Backscattering Technique for Large Fusion Devices", Rev.Sci.Instrum. 55 (1984) 457.

10. C. Gowsrs, et.al., "First $\mathrm{T}_{e}$ Profile Results from the JET Lidar-Thomson Scattering System", in the Proc. of the 14th European Conference on Controlled Fusion and Plasma Physics, Madrid, Spain, June 22-26, 1987. 


\section{Maint nance in Steady Stote Devices}

The main issue in a steady-state device is availability. If radiation and acivation were not an issue, high availability could presumably be achieved by a slight modification to a basically sound design during the start-up or shake-down phase. However, DT operation quickly activates the device and maintenance and upgrades become exercises in remote handling. In a well designed, reasonably reliable machine, availability is determined by those operations which require access to the machine since remote handling is the ime consuming, rate limiting process. Overall availability is determined by the normal maintenance activities. The difference between an INTOR-type device and a steady-state device are minimal from a maintenance perspective as the activation and remote-handling requirements are sufficiently similar that any study of maintainability on a reasonably available DT machine would be directly applicable to a steadystate device. 


\section{Pan C}

Technical Assessment of Critical Issues for Steady State Operation

Steady State Issues in Alternative Concepts

Contributors

J.N. Downing, Chairman

P.G. Weber

G.A. Wurden

B.L. Wright

C.G. Bathke

K.F. Schoenberg

* Los Alamos National Laboratory, Los Alamos, NM 87545 


\subsection{REVERSED-FIELD PINCH (RFP)}

References 1-8 contain general reviev materlal from which specific subject references to the extensive Reversed-Field Pinch (RFP) literature can be obtalned. Special acknowledgenent should be made to Ref, 8-16 which have been used extensively for sectlons of this technical assessment; these documents provide useful supplements to this assessment.

\subsubsection{Introduction}

The RFP is a high- $\beta$, axisymetric, toroidal magnetic confinement system that uses both toroldal $\left(B_{\phi}\right)$ and poloidal $\left(B_{\theta}\right)$ magnetic fields to confine hot plasma in a near-minimum energy state. RFP confinement relies strongly on the pololdal self-field generated by toroldal currents and requires only madest external toroidal confinement fields. As in cokamaks, equilibrium may be provided by efther externally applied vertical and horlzontal fields, a conducting coroidal shell, or some combination of both. The RFP may require a conducting shell to stabilize HHD modes with wavelengths longer than the shell radius, $r_{v}$. Stability at high beta (0.1-0.2) results from high magnetic shear and a conducting shell. The pitch parameter, $q$, for the RFP is low on axis, - 0.2 , and decreases vith minor radius, passing thruugh $q=0$ and becoming negative $f n$ the outer regions of the plasma. This results in two fimportant features for the RFP. The flrst is that there is no constraint imposed on the aspect ratio; therefore, it can be chosen to optimize the engineering design. The second is that the toroidal current density can be high; therefore, the RFP offers the potential to achieve ignition by ohmic heacing alone. Auxiliary heating components are not necessary. Since the poloidal finld decreases with increased distance from the plasma, both the magnetic field at the coils and the current density in the coils for a given $A B^{2}$ in the plasma are reduced compared to schemes utillzing the toroidal field for confinement. Suparconducting coils сал be replaced with normal conductors uhile majntaining a lou recirculating power fraction.

RFP discharges las: at least an order of magnitude longer than would be expected tased on resistive diffusion. This "dynamo" action is currently understood in terms of the Taylor theory; the RFP maintains a near-mirimum energy state by a continual self-relaxation process. The process of selfrelaxation may allow the use of a low-frequency ( $6100 \mathrm{~Hz}$ ) oscillating Field Current Drive (OFCD) $+11,2,1$ t technique to orjue steady-state plasma currents in a reactor. 
As a system that can potentially operate effictently (1.e., low recirculating poice) at high neutron first-vall loading $\left(10-20 \mathrm{mu} / \mathrm{m}^{2}\right) \mathrm{vith}$ exoblanket resistive colls, the RFP can possibly have fusion-power-core (FPC) power densities in the range of $10-15 \mathrm{MHt} / \mathrm{m}^{3}$ or mass pover densities of $\geq 500 \mathrm{kHe} / \mathrm{tonne}{ }^{1 /}$ This fusion system may produce cheaper electricity uhile aintaining the reactor plant equipwent as a mority part of the total direct cost. Por a radiation life fluence of $15 \mathrm{MHyr} / \mathrm{m}^{2}$ (14.1-HeV neutrons), the 20-KW $/ \mathrm{m}^{2}$ CRFPR system would operate for 0.75 full-pover years, before change out of the 45-tonne first-vall and blanket system. If the 20-MW/ ${ }^{2}$ high-poverdensity (HPD) design can be technically achieved, a single-plece or "batch" maintainance scheme may be possible, wherein a completely assembled and pretested first-vall/blanket/shield/toroldal-field-coll unit vould be installed In the reactor hall after off-site fabrication and quality assurance. In addition, a possibility exists for a shortened inftial construction and installation period, and the potential for a reduced mean-time-to-repair and more rellable reactor restarts can be traded off with the possibility of decreased mean-time-to-fallute. High plant avallability and reduced costs may be posstble. This attribute and the related adyantages associated with sinall, single-unit FPCs make the higher vall-loading designs atractive, in that the cost of electrletty per se is a veakly diminishing function of wall loading above - $10 \mathrm{HiH} / \mathrm{m}^{2}, 1$ e

The number of reversed-field pinch experiments has grown dramatically in recent years. A list of the present experiments appears in Table 1.1.1-1. of these experiments there are five which have been operating for a sufficlent time to allou an extensive data base to be accumulated. These are ETA BETA II (Padua, Italy), ZT-40H (LOS Alamos), HBTX (Culham U.K.), TPE-1R(H) (ETL Japan), and OHTE (GA Technologies). Dimensions and plasma parameters for these experiments appear in Table 1.1.1-2. The present-day RFP experiments have reached reactor beta values, $500 \mathrm{eV}$ temperatures, bnd plasma sustainment times over $30 \mathrm{~ms}$. Extended flat-topped toroidal current pulses vere attalned in 2T-40M after correcting for fleld errors, applying equllibrtum correcting fields with feedback, and with vall conditioning. The electron temperatures and densities have each been observed to scale approximately linearly with toroldal current, $I_{\phi}$, for operation with the ratio of toroidal current to plasma line density held nearly constant. In $2 T-40 \mathrm{M}$ the relationship of $\mathrm{n}_{\mathrm{e}} \mathrm{T}_{\mathrm{e}^{\prime}} \mathrm{I}_{\phi}{ }^{2}$ appears nearly constant, over the normal operzing range of $n_{e}$ and $I_{\phi}$, corresponding to 
a beta limit. The Ohmically Heated Toroldal Experiment (OHTE), on the other hand, has simultaneously achieved high electron temperature and electron densi:y when the walls are preloaded wth gas before the discharge. This results in higher total electron beta values as seen In Table 1.1.1-2. It is noted that preliminary OHTE reactor studles Indicate the need for approximately twice the beta of a standard RFP because of the higher fields associated with the OHTE helical colls. The data on the other newer variations from the standard RFP are very limited and more research is needed to determine the optimum equilibrium configuration from the reactor economics point of view.

As can be seen in Table 1.1.1-2 and Ref, 8, $\tau_{E}$ values up to $-0.6 \mathrm{~ms}$ and $n \tau_{E}$ products up to $10^{17^{-3}}$-s have been obtained. Scaling of $n \tau_{E} \propto I_{\phi}^{5 / 2}$ has been reported on $Z T-4 O H^{3}+13,2 s$, and the relationship $T_{e} * B_{p}^{-4 / 5}(\pi \tau)^{2 / 5}$ has been reported in oHTE. These scalings are favorable for the reactor as plasma parameters are extrapolated to the reactor regime.

The new RFP experiments in the design and planning stages include RFX $(a / R=0.485 \mathrm{~m} / 2.0 \mathrm{~m}, I=2 \mathrm{MA}$ ) at the University of Padova, Italy, MST $(\mathrm{a} / \mathrm{R}=0.35 \mathrm{~m} / 1.56 \mathrm{~m}, \mathrm{I}=0.4 \mathrm{MA})$ at the Unlversity of Wisconsin, and CPRF/ZT-H $(a / R=0.4 \mathrm{~m} / 2.15 \mathrm{~m}, I=4 \mathrm{MA}$ ) at Los Alamos.

\subsubsection{Plasma Control7-11,16,19}

Macroscoptc Equilibrium and Dynamics. The equilibrium for the toroidal RFP configuration consists of a plasma column containing both toroidal and poloidal magnetic fields. The former component follows the plasma column while the latter encircles $1 \mathrm{t}$. The field-plasma configuration is illustrated in F1g. 1.1.2-1. The direction of the toroidal field is reversed in the ourermost region of the plasma glving a field configuration having high shear and favorable fluid stability properties. The configuration allows global stability, with high ohmic heating currents, at high beta and with arbitrary aspect ratio.

The standard RFP configuration 15 axisymmetric and has a circular cross section. US experiments are ZT-40M and ZT-P at Los Alamos, the Reversatron at the University of Colorado, and MST at the Unfversity of Wisconsin. There are several varlants of the standard RFP. Experiments in the $U$ s that have noncircular cross sections include OtTE and Multipinch at GA Technologies (having additional helfcal windings and multiple plasma columns, respectively), and Torsatron (operated as an RFP) utllizing added octupole flelds at the University 


\section{PRESENT RFP DEVICES}

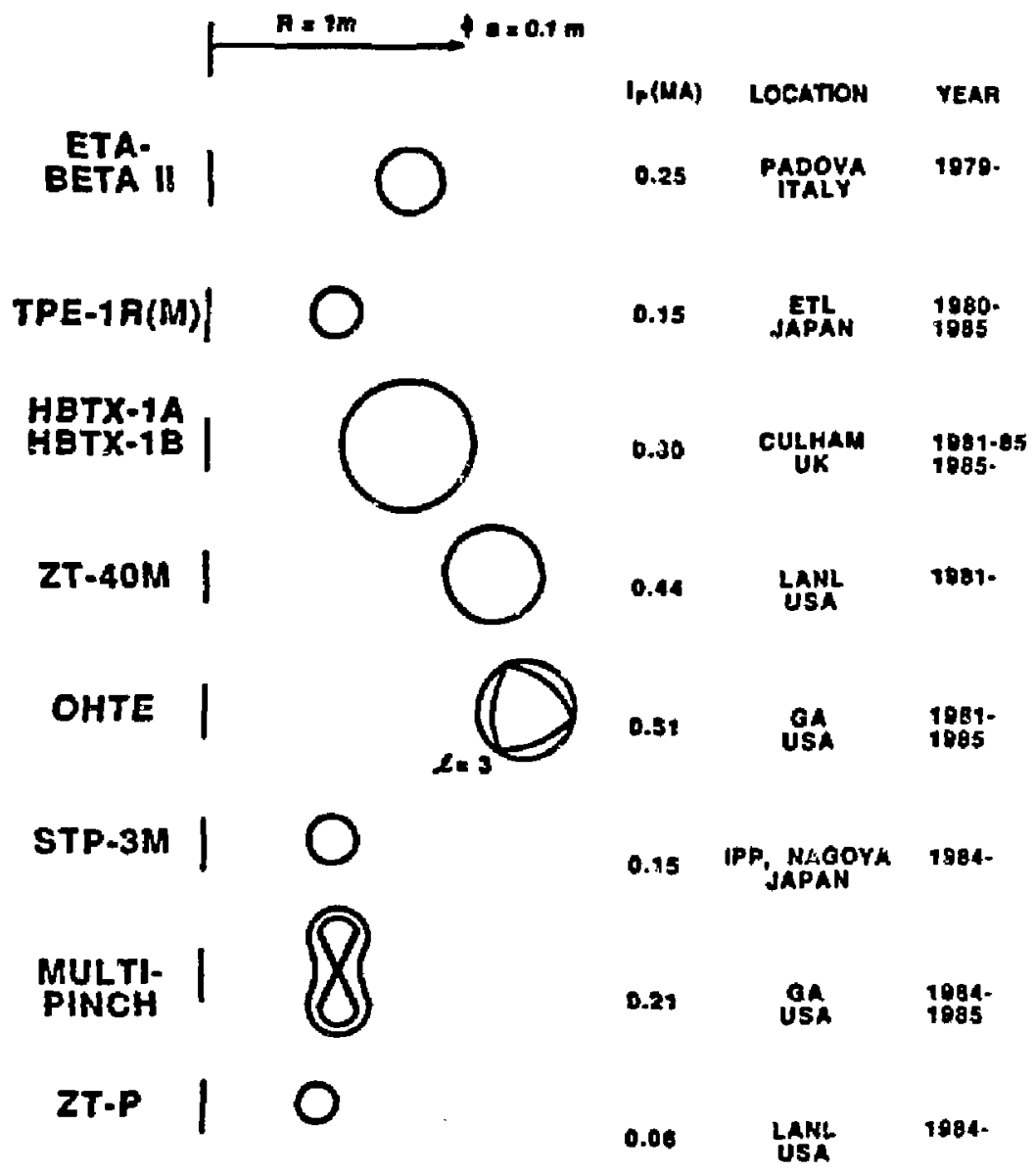

Table 1.1.1-1.9 


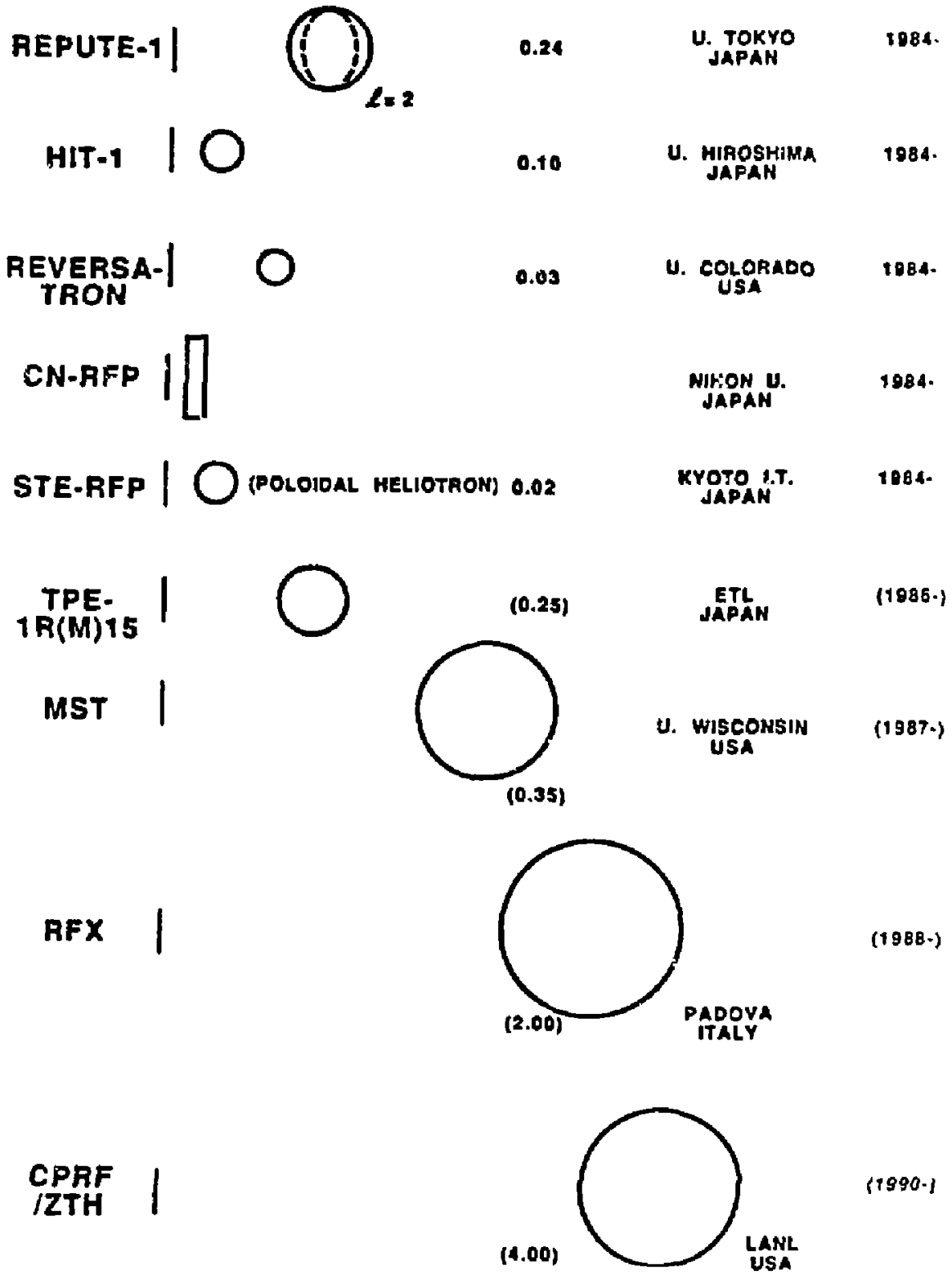

Table 1.1.1-1. (cont inued) 
TABLE 1.1.1-2."

RFP EXPERTHENTS MTTB EXTENSIVE DATA DASES

PARAHETER

R-Hajor Radius (a)

a-Minor Radius (a)

Peak Current (kA)

$B_{\theta}(a)(T)$

$7\left(1020 m^{-3}\right)$

(J) $\left(\mathrm{MA} / \mathrm{m}^{2}\right)$

$T_{e}$ (on axis) (ev)

Pololdal Beta

Discharge I/N $\left(10^{-14} \mathrm{Am}\right)$

$\tau_{E}$ (ns)

$\bar{n} t_{2}\left(10^{15} a^{-3}-5\right)$

$\begin{array}{cclll}\text { TPE-1R(M) } & \text { ETA-BETA II } & \text { 2T-40M } & \underline{\text { OHTE }} & \text { HBTX-1A } \\ 0.5 & 0.65 & 1.14 & 1.24 & 0.8 \\ 0.09 & 0.135 & 0.2 & 0.18 & 0.25 \\ 130 & 250 & 440 & 500 & 320 \\ 0.42 & 0.24 & 0.44 & 0.53 & 0.25 \\ 0.3 & 0.2-1 & 0.4-0.9 & 0.5-3.0 & 0.15-0.7 \\ 5.1 & 3 & 3.5 & 6.4 & 1.5 \\ 550 & 80-300 & 300-500 & 350-580 & 200-300 \\ -0.1 & -0.1 & 0.1-0.2 & 0.1-0.4 & 0.08-0.2 \\ 12.7 & 2-15 & 3.7 & 1.0 & 7.5 \\ -0.1 & -0.1 & 0.3-0.7 & -0.4 & 0.1-0.28 \\ -3 & -10 & 12-60 & -100 & \sim 0.6\end{array}$

Beta values assuging flat plasma energy denstity profiles.

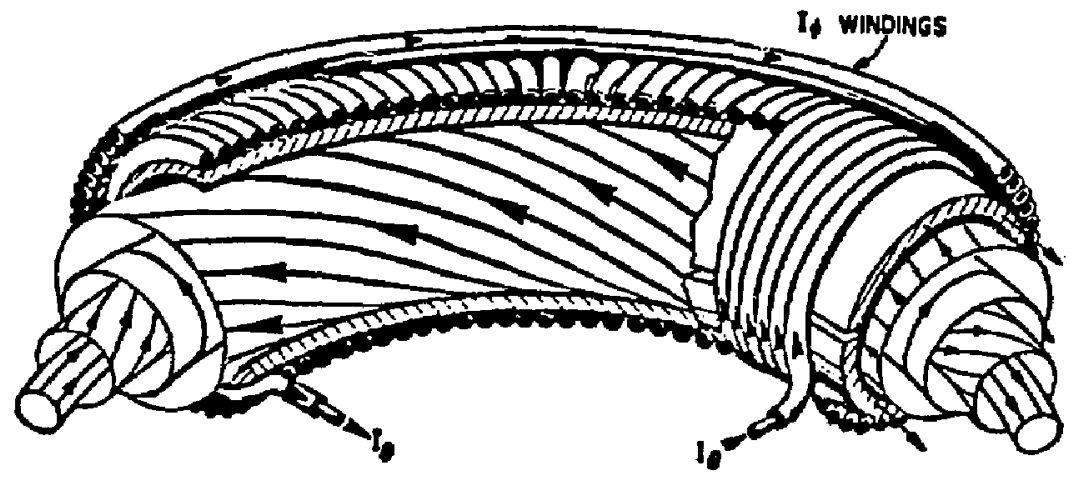

F1g. 1.1.2-1."

Schematic of showing the nested flux surfaces in the RFP configuration. 
of Wisconsin. Standard and varfant RFP experiments are befing studied in Europe and Japan and are tabulated in Table 1.1.1-2:

The early experimental and theoretical studies assumed that RFP equilibrja vould have lifetiaes governed by resistive field diffusion and with fluctuations at the therwal level. It has been observed, however, that the modern RFP experiments produce plasna configurations that last much longer than predicted by axisynnetric resistive field diffusion calculations, end the associated fluctuations are above the thermal values. Instead of decaying, the field configuration is arintalned against resistive diffuston by a process which has been termed the "dynamo effect". A present viev is that an nverage RFP equilibriun is maintained by a self-field reversal dynamo mechanisw with the plasma continually relaxing to a preferred state vhich is near the state of Inimum energy (Taylor state). Experinents indicate the configuration can last as Iong as the plasna density and toroldal current are maintained. Recent work suggests that the RFP current configuration may be maintained indefinitely in a mean steady state by modulating the fields at lou (audio) frequency, vith equilibriut control and with gas injection to sustain the density.

The theory of axisymmetric equilibria is vell in hand and the equilibriam may be modeled with present-day toroldal computer codes. The theory of the effects of nonaxisymmetric fleld errors caused by divertors, winding and shell imperfections is not yet established although three-dimensional equilibrium and dynamic codes capable of addressing these issues are being developed. A code which allous the coupling of a general equilibrium to the field colls and their clrcults yould meterially essist in the design of the feedback control system. Experinents have shown that the average equilibrium can be controlled and maintained by a sufficient reduction in the field errors and vith feedback fleld correction. The experimental observations indicate that the time-averaged equilibrium can be qualitatively understood as constantly undergoing a relaxation tourds a preferred reversed-field state.

Stabllity. The Iinear stabllity theory is vell developed and the tools exist for analyzing any given axisymmetric RFP configuration for linear stability. Linear fluid theory has been used as a guide in the search for favorable RFP configurations, contact with this theory and present-day experiments is complicated by two factors: (1) the hot, long-lifetime RFP plasmas do not allov magnetic fleld profile measurements with conventional internal probes; (2) the plasma with its dynamo action has a sizable fluctuation 
level at all times, and its description involves the nonlinear interaction of many ardes.

In the presence of a perfectly conducting shell there exist RFP equilibria which are linearly stable against all fluid wodes with one exception: pressure Jriven resistive nodes. For the nodel RFP configurations, these modes are unstable throughout the plasna. Since pressure-driven modes typlcally have andi-scale vavelengths, the turbulence they cause is a good candidate for dominating the confinemist properties. The resistive current-driven tearing mode is expected to be fuportant in relaxing globally unstable preslles toward stable ones.

The role of the conducting shell is a critical issue. As the magnetic fields diffuse into the conducting shell, the plasma equilibrium and stability w11 change. Acflve feedback control for globaj. plasna positioning (assuming not needed for local modes) vill be necessary for the stendy-state or long-pulse device. A careful assessment of the critical features and parameters (thlckness, unlforaity, penetrations, gaps, etc.) of the conducting shell is necessary. Field errors due to holes and gaps in the conducting shell and/or the flelds from afvertor colls may promote the development of stochastic field lines and magnetic islands in the outer edge of the plasma. A major goal of the current programs is to evaluate the effect of fiuld errors and to determine adequate means of correcing the errors.

The study of the atability properties of the RFP wth a resistive shell (or with no conducting shell) is an active area of theoretical and experimental research.

Transport. 2T-4OH has operated with flat-topped currents in the range of $25-400 \mathrm{kA}$ to examine the dopendence of the plasma parameters on the plasma current. 46,2 . The functional dependence of plasma poloidal beta, $\theta_{\theta}$, and energy confinement $\left(\tau_{E}\right)$ are sensltive to the parameters, if any, that are held constant. The plcture is further complicated by the observation that the ine average electron density, $\bar{n}_{e}$, and the electron temperature, $T_{e}$ measurements off-axis indicate a flattening of the pressure profile at the higher currents. For example, at a radius of $14 \mathrm{~cm}, \bar{n}_{e} T_{e}$ is about two-thirds of the value on-axis at a plasma current, $I$, of $120 \mathrm{kA}$. At $300 \mathrm{kA}$ the $\vec{n}_{e} T_{e}$ product at the same radius was -0.9 of the on-axis value. $2 T-40 M$ was operated vith currents in the range 60-400 ks vith a ratio of plasma current divided by line censity $\left(N=\pi a^{2} \bar{n}_{e}\right), I / N$, whlch varjed over the range of $2.5-7.0 \times 10^{-14} \mathrm{~A}-\mathrm{m}+16$ The 
plasma polofidal beta, $\hat{B}_{\theta}$, derived from $\bar{n}_{e}$, central electron temperature, $T_{e}(0)$, and the assumption of $T_{1}=T_{e}$, was constant over this range of plasma currents. The energy confinement time, $\tau_{E}$, vas estimaced froin the ratio of the plasma energy, $w_{p}$, to the input power, $P_{1 n}$, and the resulting product of $\tilde{n}_{e} \tau_{E}$ was proportional to $\mathrm{I}^{2.2 \pm 0.4}$.

In another set of discharges 2T-4OH vas operated as an RFP vithout $11 \mathrm{~m} 1 \mathrm{ters}$ in Elat-top current node ranging fron $25-400 \mathrm{kA}$, with flat-top durations of 3-30 ms.19 The shorter duration occurred at the highest currents. A downuard trend in $\tilde{n}_{e} T_{e}(0) / I^{2}$ was seen as current was Increased whlle $I /\left(N / T_{e}\right)$ vas held roughly constant. This flat profile estimate of $s_{\theta}$ was $-8-15 \%$ it $I_{\phi}-350 \mathrm{kA}$, but ranged from $-20-40 \%$ at $70 \mathrm{kA}$. At each current level, It was still observed that cemperature and density were inversely related, and at high currents, $B_{\theta}$ values vere similar to previous scsilng data. significant changes in the profiles (broader and flatter) occurred when the current was ralsed, and large sartooth osclilations appeared which were not present at the same $F$ and $\theta$ at the lower currents. These two effects complicate the scaling results, which vill require both better profile, knovledge (multi-point Thomson scattering), and better profile control (gas puffing and pellet injection) to clarify the physics and thereby the scaling predictions. clearly, the understanding of the physics which governs $\theta_{\theta}$ and $\tau_{E}$ are critical issues.

Current terminations must be understood and controlled in any RFP reactorlike device. Very large amounts of energy (kinetic and magnetic) can be deposited at high pover levels on In-vessel components if the current is allowed to terminate too quickly. Controlled ramp-doun of the current has been accomplished in 2T-40H; however, most discharges are alloved to end vith a current termination. The key to reactor operation lies in understanding the physics so that controlled ramp-down of the current can be achieved under all possible operating modes.

Profile Control. To permit a serious comparison of theory and experiment In the arsa of radial transport, a fusion concept must have matured to the point tha: transport is not dominated by extraneous effects. Experimentally, this requires plasma equilibrium position control, understanding or elimination of magnetic field errors, impurity control and other operational features. Theoretically, a description is required of the plasma equillbrlum and certain macroscoplc stability properties. In recent years the RFP has attained a significant siatus in this regard. Nonetheless, the RFP shares, vith 
essentially all other fusion concepts, the property that the transport of energy across magnetic surfaces is anotalous for reasons which ere unknown.

The theoretical ainimum Ievel of transport in a toroldal RFP is low because the neoclassical enhancenent has a negligible effect on the transport (above that for a cylinder) oulng to th: veak pololdal esymatry of the RFP in contrast with that of a tokanak. Yet, similar to the tokamak, the measured global transport in the RFP exceeds the classical minimum by an order of magnitude. The crucial goal of RFP fuston research is to attain a theoretical understanding and experimental determination of scaling of transport with plasma parameters as the reactor regine is approached.

The RFP is distinctive in that a prerequisite to an understanding of the transport is a valld description of the sustainment of the equilibrium. As mentioned in a previous section it appears that there exists a preferred globally stable reversed-fjeld state and the plasma tends to evolve avay from this state by resistive diffusion, but the "dynamo" mechanism continualiy returns the plasma to this desirable state. This mechanism di manifested as broad-spectrum magnetic turbulence.

In addition to sustaining the clasma, the magnetic fluctuations may cause stochasticity of the field and enhance the radiel transport of energy. However, some theorjes predict that the fluctuation amplitude is proportional to an inverse fractional pover of the magnetlc Reynolds number $S\left(e . g ., B / B-s^{-1 / 2}\right.$ ) where $s-T^{3 / 2} B / n^{1 / 2}$ and $T, B$ and $n$ are plasma temperature, magnetic fleld strength, and plasme density. This scaling of the fluctuation level, which is somewhat supported by existing experinents, predicts a favorable confinement time scaling with $\tau_{E}-s-T^{3 / 2} B / n^{1 / 2}$. Experimental correlation between dynamo activity and confinement has not been demonstrated. In fact operation of the RFP in a regime thich requires a large amount of toroldal field generation has not shown a significant average energy confinement time degradation compared to discharges with a small amount of fleld generation.

The plasma current is often considered to be the free energy =ource which drives the magnetic turbulence suspected to be responsible for the dynamo and enhanced transport. However, it is also observed that under normal operating conditions, essentially all RFPs characteristically have high beta limits (the ratio of plasma pressure to magnetic field pressure). These have of ten been assocfated with a stability $11 \mathrm{mlt}$, as predicted by linear stability analysis of certain pressure-driven MKD modes. The reality of this interpretation, as well 
as the relative contribution to transport of pressure-driven versus currentdriven instabllities, remalns an open question.

An important consideration is the possible contribution to transport of ujcroscopic instabilities and turbulence, such as those generated by drift vaves. The drift vave problem is similar in RFPs and other devices, albeit the distinctive features of the RFP is its high magnetic shear and its relatively large ratio of electron drift speed to electron thermal speed.

Progress in RFP transport physlcs requires both more detalled fluctuation measurements and expanded operatiog regimes. Operation at significantly higher 5 values (which requires higher temperature and plasma current) is critical to test transport theorles. The dependence of the $B$ limit on plasma temperature and current must be more stringently tested to establish whether the present apparent IIwit is universal. The RFP has not yet had the opportunity to operate over a sufficiently broad range of parameters for the empirfcal tracking of transport that has proved essential in the evaluation of the feasibillty of cokanaks and/or mfrrors. Thus, the future improved experiments hold great promise for the generation of information which vill critically bear on the general problem of trassport in current-carrying toroidal plasmas.

Alpha-Particle Effects. The DT-generated alpha particles must assume the major role of plasma heating (taking over from the ohmic heating in the case of the RFP) while not exciting undesirable plasma instabilities of either an MHD or thermal nature. As for most magnetic fusion devices, achievenent of an alphaparticle-dominated (1.e., ignited) plasma requires that the thermalized alpha particles leave after depositing $280 \%$ of their energy in the bulk plasmas thereby keeping the hellum population on the average of $\leq 5 \%$ that of the DT fuel. A fraction of the virgin 3.5-HeV alpha particles in a toroidal confinement device will be trapped in the magnetic mirrors formed by the natural high toroldal flelds at the inboard of the torus compared to the outboard regions. These trapped alpha particles hav a banana-orbit width $\delta$ compared to the plasma radius, $z_{p}$, given by:

$$
\frac{\delta}{r_{p}}=\frac{1.91 \varepsilon^{1 / 2}}{I_{\phi}(H A)\left[1+(\varepsilon / q)^{2} 1^{1 / 2}\right.} .
$$

where $\varepsilon$ is the Inverse aspect ratio and $q$ is the tokamak safety factor. In 
contrast wth the tokamak and where $q / \varepsilon \geq \theta$ and this fraction can be large (> 0.1 for $I_{\phi}-10 \mathrm{MA}$ ), for the RPP with q/e $\approx 0.08$ retention of 3.5-HeV alpha particles should be a liss serlous concern $\left(\delta / r_{p}=0.007\right.$ for $I_{\phi}=10 \mathrm{HA}$ ). classically, the a particles will be confined in an RFP. The effect of turbulence, hovever, on the $\alpha$ particle contalnment has yet to be explored. The zeneric nature of alpha-particle physics, unfortunately, cannot be extended much beyond this point In that the unique field profiles in the dynamo-sustained RFP plesma are required to address the plethora of alpha-particle issues that face magnetic fusion in general and the RFP spacifically. A few of these issues are summarized below.

- Impact of plasma stability and confinement upon transition from a heavily ohalcally heated plesma (strong heating in the outer plasme regions) to one that is alpha-partfcie heated (strong central heating).

- Changling fleld profiles - Impact on RFF dynamo - Impact on dynamo-related processes.

- Alpha-particle-driven microturbulence and enhanced transport.

- Degree of anomalous alpha-particle thermalization and shift in heating avay from the electrons and more towards the ions.

- The magnitude of the suprathermal alpha-particle population and impact of associated pressure on beta and bete limits.

The majority of these issues mist be addressed with an RFP plasma/field configuration.

If the RFP physies scaling continues to project a plasma that can operate at a reactor-relevant beta IImft, then the impact of alpha-particle-driven beta excursions will be one in which the plasma confinement is degraded if the burn intensifles. Recent burn simulations of ignited RFP plasma for both reactor and near-term devices have invoked a soft beta $11 \mathrm{mlt}$ of the form $\tau_{E} \propto\left(\beta_{\theta c} / \beta_{\theta}\right)^{2}$ if $\beta_{\theta}$ exceeds a critical value $\beta_{\theta c}=0.1$. Other methods of burn control that are relevant to RFPs vould increase the plasma HHD activity by control of magneticisland size (1.e, field ripple) or increase the streaming parameter $\left(\varepsilon=j_{\phi} / n T^{1 / 2}\right)$. 


\subsubsection{Pueling $16,1,-24$}

In the next generation devices (RFX and CPRF/ZT-H), a combination of vall conditioning, hydrogen recycling from graphite, distributed gas puffing, and pellet injection w11l be used for density control. In reactor systems pellet infection will be used for fueling and profile contral while gas puffing will b: used for edge plasna control.

Gas Blanket. Gas puff refueling in the RFP has been demonstrated on several machines. The fueling requirements decreased and particle confinement was Improved when ffeld ercors were reduced in $2 T-40 \mathrm{H}$. Although gas puffing increased the plasna density throughout the entire plasma cross section, the number of particles contributed to the plasma was less than the number injected. In $2 \mathrm{~T}-4 \mathrm{OH}$, gas puffing has increased the dfaneter averaged plasma density by factors as large as 2.5 for $120 \mathrm{kA}$ discharges. Flat or hollow density profiles are produced, especially as $\bar{n}_{\mathrm{e}} \mathrm{a}>10^{15} \mathrm{~cm}^{-2}$, as in tokamaks. The increase in density produced a concowtent decrease in central electran temperature because the maximum beta remained relatively constant.

The penetration depth for gas puff refueling is expected to scale inversely wth increasing density; therefore, pellet injection will be implemented for Improved refueling and profile control in larger higher denstiy devices. Edge-plasma fueling can be used to lower the plasma edge electron temperature and give better protection of the first-vall and in-vessel components from sputtering and erosion. This fueling nethod is usable primarily in confunction with punp limiters.

Pellet Infectlon. Pellet refueling requirements for both the RFP and tokamak are similar. The pellet lifetime is veakly dependent on average plasma density $\left(\propto 1 / n^{1 / 3}\right)$. The decrease in plasma radius for RFP HPD systems more than compensates for the higher plasme density and the shorter particle confinement times and results in pellet velocity requirements similar to the larger tokamaks. It is topic of present speculation, that penetration beyond the revetsal layer in an RFP may be adequate, rather than all the way to the axis. Systens with similar energy outputs will require similar fueling rates; therefore, the pellet injection frequency should be the same.

The first pellet refueling in an RFP22 has encountered an interesting twist, apparently as a result of the high drift parameter $\xi=v_{\text {Drift }}^{e} / v_{T h}^{e}=0.1$ in present day experiments. Curvature of pellet trajectories is serious enough that careful aiming of the pellet infector is necessary to compensaze for 
deflections that can otherwise prevent approach of the pellet to the plasma axis. This effect should be smaller in larger, hotter devices if $E$ is lower.

There are prelininary indications that today's tokamak-pellet ablation codes $^{23}$ using thermal ablation models over estiate penetration Into the RFP, if best estimate density and temperature profiles are chosen.

\subsubsection{Impurtey Control7-16,23,25-32}

Current generation RFPs operate vell within the $z_{\text {eff }}$ for lov- $Z$ impurieles required for a reactor. Changing the first vall to low 2 materials will eliminate the high $z$ impuritles present in these devices. Simllarly, lov $z$ will probably be the choice for plasma-side wall materials in future systems. The cholce of material for in-vessel components, the control of edge-plasma conditions, and the implewentation of active impurity control by pump limlters or magnetic divertors will be necessary to minfoize plasma contamination for the longer-pulsed devices.

Limiters and surface conditioning are the impurity control techniques presently used in RFPs. The conducting shell and the vacuum iner in most RFPs are concentric. The equilibrium shift of the plasma colum causes the liner (including limiters or armor) on the outside major radius to act as a single, coroldally continuous, limiter. The liner material is the major source of impuritles. The choice of this plasma-side materlal and the implementation of impurity control techniques are important issues for future devices. The OHTE has operated with 100X graphite coverage in the RFP mode, and HBTX-1A(B) (Cultam) operates with $10 x$ graphite coverage.

The high-power-density operating mode of the RFP results in two distinguishing features for the liaiter/first-vall: (1) a large percentage of the first-wall must serve a limiter function, and (2) large peaking of the heat flux cannot be tolerated. Both arrays of pump limiters'1011-14,16 and coroidal-field magnetic divertorsind11,12,14-16 are being considered for impurity control. Hagnetic divertors and pump limiters have been evaluated for both the next-generation device at Los Alamos (CPRF/2T-H) 16 and the Compact Reversed-Field Pinch Reactor (CRFPR), $7+11+13+24$ and most recently for the Titan/RFP.11 Poloidal pump limiters can be used if the plasma stability vill allow a relatively Iarge separacton between the limiter minor radius and a conducting boundacy which is toroldally and polotdally continuous. A coroldally-segmented, pololdally-continuous linlter wlll not stabllize all MHD modes. Whereas the large separation (and corresponding large e-folding length) 
Is destrable from the plasa engineering point-of-vleu, it may not be sufficient for plasma stability. If short connection lengths betveen limiters are required to reduce the e-folding lengths (heat and particle flux) and thereby minimize the separation, toroldal pump liniters ( 1 or more) can possibly be used, provided the extreme heat flux on the edge of the lificer can be avolded. A large-area pump limiter concept ${ }^{25}$ has been proposed which requires the least changes fros present experimental conditions in RFPs with respect to modiflcations of the conducting boundary, the limiting configuration, and the agnetic field geometry. A pololdally symetric toroldal field magnetic divertor appears to be very attractive for Impurity control; however, unresolved Issues are plasma stability with large toroidal and poloidal gaps in the conducting boundary (q-reversal issue)1sit and plasma stability with the non-axisymetric fleld perturbations from the divertor configuration.

Plasma-Edge Conditions. Reversed-field pinches have historically been formed in vacuum vessels in which no effective liniter strucilires vere employed. Recent efforts have been made to operate RFPs with a variety of limiter configurations, $25+2 \% 29$ or with the vacuum vessel completely shlelded by graphite tiles.30 while these experiments have shown sone success, the characterization of the edge plasma vith these Iimiters has not yet been documented. It is to be anticipated that the plasma conditions to be found in the lintter shados of an RFP vould not be greatly different to those found in a tokamak wth equivalent particle and energy fluxes.

Significant data are avallable from the ZT-40M RFP operated without limiters.31,32 Specifically, a double Langmulr probe has been used to measure the plasma electron temperature and density at a radius equal to the minimum radius of the vacuum liner (the diagnostic sectlons of the liner are at larger radii than the innermost polrts of the Inconel bellows that comprise the remainder of the vacuun vessel). In short duration (5 ms) discharges with total toroidal current of $120 \mathrm{kA}$, the electron temperature vas $45 \mathrm{eV}$ at a density of $5 \times 10^{18} \mathrm{w}^{-3}$. This relatively high temperature implies ef ther a more conductive edge plasma than is typically found in tokamaks, or a deviation of the magnetic fleld Iines at the diagnostic station due to coil winding errors around the large port. This probe also determined the direction of impact for electrons to be nearly poloidal in the RFP edge, with a drift velocity of several percent of the electron thermal speed; no ion drfft vas detected. By measuring the heat flux and current collected by another edge probe, it was inferred that a 
population of suprathermal electrons exists in the ZT-4OM edge plasma. Fur ther evidence for this comes from deflection of frozen pellets of hydrogen or deuterium, 3 from analysis of liniter damage, from a Sill detector and from a neutral partlcle analyzer. The suprathermal population can be suppressed by gas puffing, or, very effectively, by peilet fueling of the plasma.

Temperatures of neutral deuterium atoms and oxygen impurity fons in the edge plasma have been obtained from Doppler broudening of fmpurity lines.3 1 The optical system used restricted the view to be at nost $4 \mathrm{~cm}$ from the vacuum vessel vall. Ion temperatures in the range 10-20 eV were weasured.

The same spectroscopic system 2 vas used to look for mass flow in the edge plaswa; propagating fertures are observed fram electron density measurements and from magnetics data. For times less than $5 \mathrm{~ms}$, upper $11 \mathrm{mits}$ to any edge mass flow of $6\left(10^{3}\right) \mathrm{ms}^{-1}$ toroidally and $7\left(10^{3}\right) \mathrm{ms}^{-1}$ pololdally vere obtained. These linits are much smaller than the velocitles observed from the propagation of fluctuations seen by the interferometers and the magnetics; hence these edge modes represent a phase velocity rather than a mass flow. (At times greater than 5 ms plasma interactions Hth a prism inserted into the plasma edge to make the measurement were too severe to obtain reliable data.)

The particle confinement time in $2 T-40 \%$ has been inferred from absolutely calibrated measurements of D-alpha emission to be typically less than 1 ms. Consequently, the particle flux to the vall is larger than in a typical Tokamak of equal dimensions; control of this flux is clearly of great 1mportance. Experiments have been performed with four graphite poloidal rings in $\mathrm{ZT}-40 \mathrm{M}$; while these rings have an ineffective topology for fleld line interception in the RFP, a factor three increase in recycling was observed at the 5 ings.32 Further experiments on Ifmiter configurations are planned for $2 \mathrm{~T}-40 \mathrm{M}$.

RFP plasmas have also been formed in a vessel lined with graphite tiles.30 Since the graphite can hold a large quantity of hydrogen, the recycling in these experiments was much faster than wh a metal vall, resulting in operation at generally higher densitles. A graphite vall is also planned for the multi-megaampere RFP experiment 2T-H to be performed in the Los Alamos CPRF. Results from these experiments are expected to provide an lmproved basis for wall design in reactor level situations. The combination of relatively short confinement times and compact dimensions of RFP reactor designs' I may require Innovative sol.tions to wall protection in such devices. 
Pumped Limiters. The possibility of using pumped limiters in the CRPPR' 1"11 has been examined. For plasma conditions that allow $90 \mathrm{x}$ of the ohmic and alpha-particle pover generated within the plasma to be radiated to the first-uall/linter surfaces, 24 pololdal puaped liniters covering approximately 38 of the first-vall area vould allow $21 \mathrm{X}$ of the plasma particle loss to flov Into the linter slot while malntadning the energy density onto the limiter leading edge below $6 \mathrm{mH} / \mathrm{n}^{2}$. Figure 1.1.4-1 glves a comparison of the heat $\mathrm{flux}$ versus 11miter area for the CRFPR and starfire designs using 1inters. By designing the CRPPR 11ater to occupy a greater fraction of the evailable firstvall area and selecting siallar radiation fractions ( $f_{R A D}=0.9$ ), the CRFPR design receives heat fluxes sinilar to thet expected for the starfire $11 m i t e r$, with the CRFPR 11aiter area, $A_{L}$, almost equal to that for starfire but with a factor or - 10 reduction in first-vall area, $A_{F W}$ and associated FPC ass and cost. The cooling requirements for the punped lialter are fimilar to those for the first wall. Copper alloy is proposed for the pumped-11miter surfaces facing the plasma although vanadiun alloys may also suffice, As seen in Fig.1.1.4-2, a pololdal orientation of the linter relative to the first vall is selected. The shape of the outer aurface of the 11 mfter vas deteralined by an edge-plasma wodel siailar to that used for tokamaks.11 The limiter radial thickness is made as small as possible consistent ith the inclusion of coolant-vater channels of sufficient size to provide adequate cooling ac aceptable coolant velocities and pumping power vhlle maximizing the limiter-slot area for purposes of particle

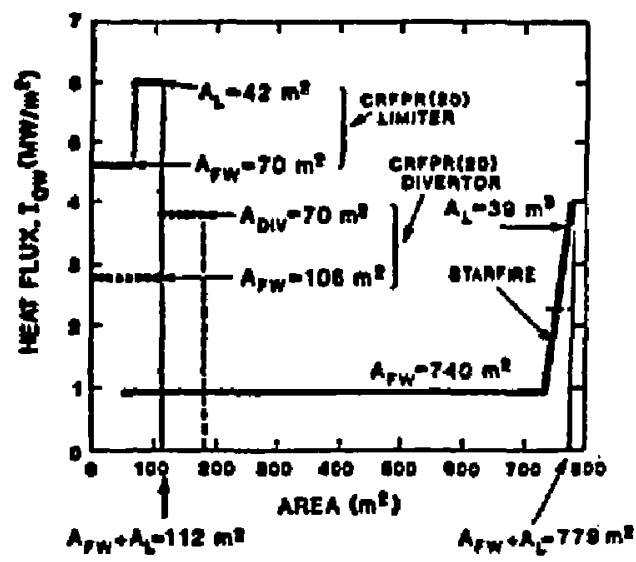

Fig. 1.1.4-1.

A comperimon of heat flux versus linlter area for a CRFPR and the starfire designs using liniters. 

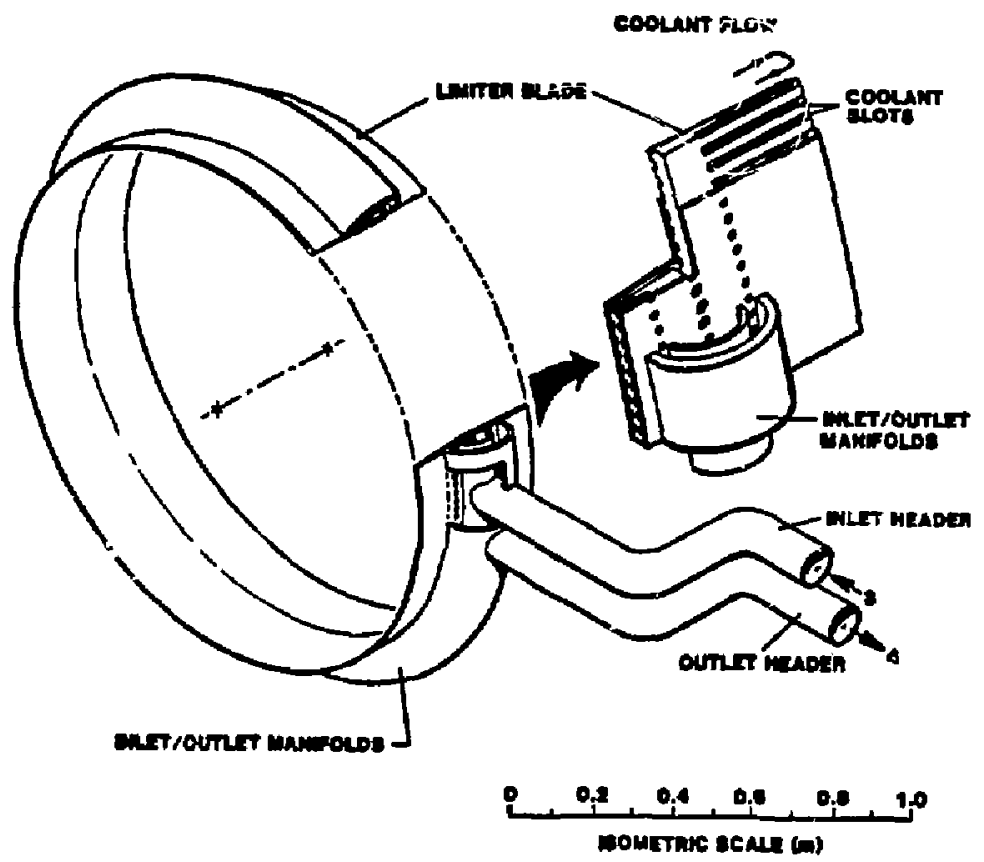

F18, 1.1.4-2.

Detalled viev of one of the $N=24$ pumped poloidal liniters and accorpanying pressurized-uater coolant circuits.

pumping. Cooling rates, heat fluxes, and geometric parameters for the limiters are summarized in Table 1.1.4-1.

A critical cooling problex occurs at the liniter tip as for most fusion concepts using this approach. The surface of the Iimiter facing the plasma receives both a radiation flux and a (unresolved) particle flux. This surface Is shaped so that the comblned energy flux incldent on the limiter surface is unifor: and does not exceed a nominal design limit of $6 \mathrm{~mW} / \mathrm{m}^{2}$. The coolant channel configuration proposed for the limiter is shovn in Fig. 1.1.4-3, which gives both heat fluxes and steady-state temperature distribution. Although the ifmiter temperatures are vell vithin the acceptable range set by creep for the configuration shown, the relatively thin sections required allow litile design 
TABLE 1.1.4-1

PUHPED-LIMITER THERMOHYDRAULIC DESIGN PARAKETERS

Gear Flux and Total Power

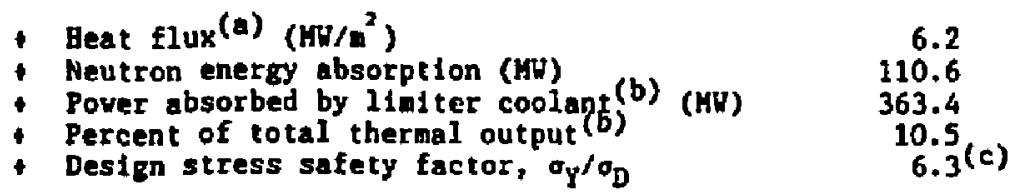

Geonetry

- Number of limiters

- Number of channels per linjter side

- Channel length (n)

- Channel vidth (min)

- Channel hight (ma)

- Vall thickness (mn)

24.

942.

0.38

4.0

0.8 (d)

(a) The plasma side of the liniter vould be shaped to maintain a nominally constant plasma heat flux.

(b) In addition, 204.8 MW of neutron and gamma-ray heating is deposited into $t$ s: inlet/outlet manifolds, giving a total of $568.0 \mathrm{kH}$ or $16.4 \%$ of the total thermal pover.

(c) Based only on pressure stress applfed to a beam-like geometry. Preliminary estinates indicate that the thermal stress is small by comparison.

(d) Although satisfactory from a stress vieupolnt, this thickness allows no sputterfing margin. Graphite tiles or Be coatings vould be used.

margin for significant net sputter erosion. The improviments needed to analyze better these important plasma-vall interactions, including self-consistent calculation of sputtering rates, redeposition, and radiation fractions, represent an important area of future work.

Hultiple toroidal pump Ifmlters have been considered for the CPRF/2T-H 6 because thej offer better protection of the liner in the event of loss of equilibrium and they require less distance between the plasma edge and the liner than poloidal ring liniters. Shorter connection lengths betveen limiters lead to shorter scrape-off lengths and a potentially more compact impurity control system; hovever, solutions to the heat flux and correspondirg heat removal probless at the edge of the limiter of a conventional design are challenging-toimpossible. 


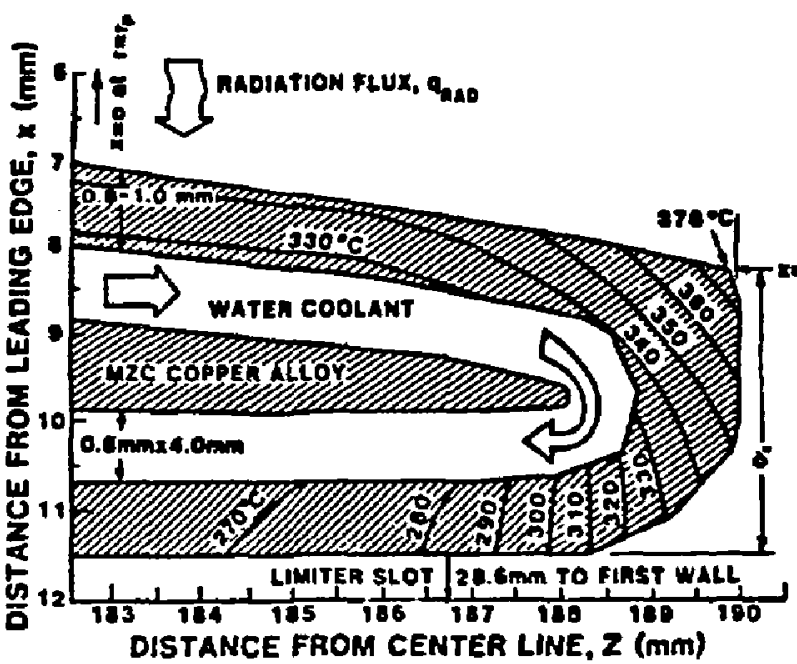

P1E. 1.1.4-3.

Linter-tip temperature and heat-flux distribution based on design developed in Ref. 7.

A lazge area pupp liniter27 has beer proposed which has potential epplication to fusion syatems, In general, and HPD systems, specifically. As 1llustrated by the cross-sectional viev shoun in F1g. 1.1.4-4, the proposed scheme removes edge-plasma particles through untformiy distributed syster of channela which perforate the first-vall/araor. The small size and uniform distribution of the pumping channels altigates many of the pump limiter destgn problems assoctated with variations in heat and pactlcle flux resulting from changes in plasal position and plasua conditions. The advantages are (1) it is effective over a ulde range of plasma conditions, (2) it is equally effective for short and long density scrapeoff lengths, and (3) it is easily adapted to a full vall armor approach there the entire first-vall is the pumping element. The displacement of the first-wall/armor waterial by the pumping channels resules in an enhancement of the heat flux on the edges of the pumping channels. The chord length across the pumping channel along each field line defines the connection length and a corresponding energy scrapeoff length characteristic of the edge-plasma In the vicintty of the channel. This enhanced heat flux can be accommodated by beveling the sides of the channel at the entrance to a depth of a few local scrapeoff lengths. The angle of the bevel increases the surface area so that the peak heat flux is kept to an acceptable level. 


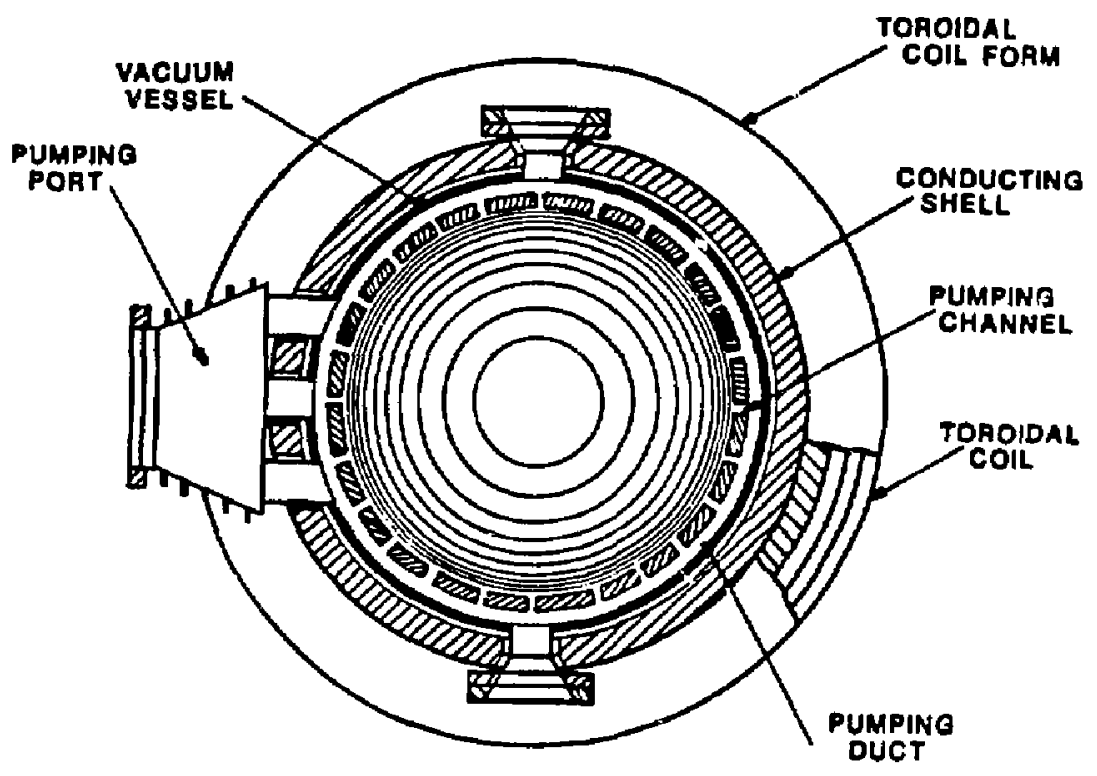

P1. $1.1 .4-4$.

Cross sectional vies of minor diameter in an RFP with pump limiter first vall.

If the plasma column shifts from the ideal position, the fleid ilnes Intercept the first-wall/arnor at a shallow angle, and the first-vill in the direction of the shift acts as a large area bumper liniter. For the large-area puap limiter, the enhanced heat flux on the sides of the pumping channel increases in the sane proportion as the local heat flux. In the absence of nonlinear density effects, the overall particle removal rate is not affected by the sinift of the plasma. Areas with higher particle flux pump correspondingly more particles, shereas, the backflou of lov temperature neutrals from the pumping duct remains the sane. If the shift is consistently many e-folding lengths, the pumping channeis can be placed only in that portion of the first-vall/armor that is in plasma contact ( $u$ th the 1 inft being a discrete toroldal liviter with punping channels).

Toroldal Fleld Divertor. Magnetic divertors are an attractive alternative to the pumped-limfter impurfty control schemes discussed above because divertors avold the vall erosion problem. In a divertor design the region of the plasma- 
vall interaction is isolated from the plasma so that contamination of the core plasma by impuriti:s sputtered from the wall becomes negligible. Hovever, while divertors have been extensively employed with considerable sucess in present tokamak experiments, no RFPs have operated with a divertor, although it is planned to eventually undertake divertor experiments in the proposed CPRF/ZT-H device at Los Alamos. The divertor has been selected as the primary approach for impurity control for the TJTAN 2 study.

Generally, the minorlty field (the poloidal field for a tokamak and the toroidal field for a KFP) is the preferzed component to sull in a divertor systen. 15 Nulling the minority field minimizes the magnetic-field perturbation at the plasma edge, mintulzing adverse effects on the pressure and particle or energy confinement times.71s The efficiency $\forall i t h$ which particles enter the divertor is increased by minimizing the magnetic mirror depth at the divertor throat, which also is accomplished by nulling the minority field. Lastly. divertor-coil currents scale linearly with the magnitude of the nullod field component; the energy stored in, forces on, and pover consumed by the divertor coils are all minimized by nulling the minority field component. Diverting the minority poloidal field in a tokanak requires that the divertor coils either be interlocked with the torvidal field coil set or reside outside the toroidal field coils, with substantially increased currents being predicted for the latter. The minority toroidal field in an RFP can be mulled without the engineering complexity of interlocking coils. Nulling the toroidal field in an RFP, hovever, does raise concerns about the stability of an RFP plasma vith a non-monotonic surface-averaged $q(r)\left(=B_{\phi}{ }_{p} / B_{\theta} R\right)$ profile, relatively large toroidal gaps in the firstwall, and a general breaking of the preferred toroidal symmetry.

Tro kinds of divejtors are best suited to the RFP magretic geometry: a toroidal-field, poloidally symetric divertor (SD) and a toroidal-field, bundle divertor $(B D)$. The $B D$ offers strong maintenance advantages associated vith a plug-in capability. A physical layout vith 24 BDs is shown in Fig. 1.1.4-5. Field line tracing indicates an inordinantly long connection length ( $1 \mathrm{~km}$ ), and most particle and associated energy loss vould be transported to the first wall. Consequertly, the $B D$ is not considered feasible for the low-q RFP configuration. Interest in the SD principally rests vith an inherently shorter connection length compared to the BD. The physical layout of a field period of 


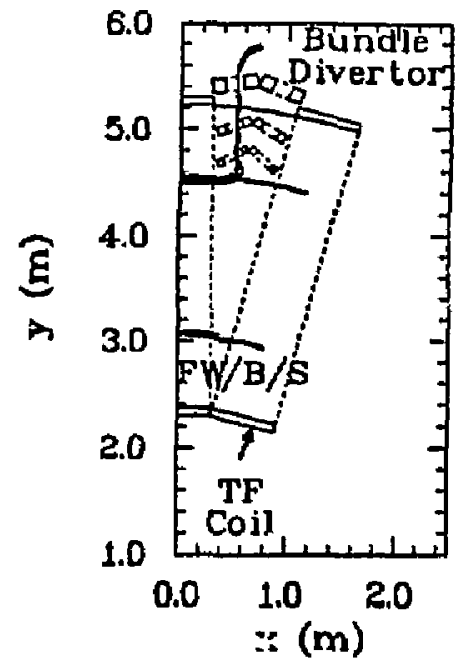

Fig. 1.1.4-5.

The tyo-dimensional field-line tracings (solid lines) for 24 ads for $r=0.69,0.705,0.715,0.73$, and $0.75 \mathrm{~m}$ on the outboard side and 0.71 and $0.75 \mathrm{~m}$ on the inboard side. Also shown is the puncture plot for the three-dimensional simulation of plasma and coils for $r=0.73 \mathrm{m.}^{3 \mathrm{~s}}$

TABLE 1.1.4-2

DIVERTOR COIL DESIGN PARAMETERS

Parameter

NULL COIL

Current (HA)

Current Density $\left(\mathrm{MA} / \mathrm{a}^{2}\right)$

Coll Radius (m)

FLANKING COILS

Current (MA)

Current Density ( $\left.\mathrm{HA} / \mathrm{m}^{2}\right)$

Coll Radius ( $($ )

OHMIC POUER (MY)
Value

0.8

50

1.088

0.4

40

0.97

48,4 


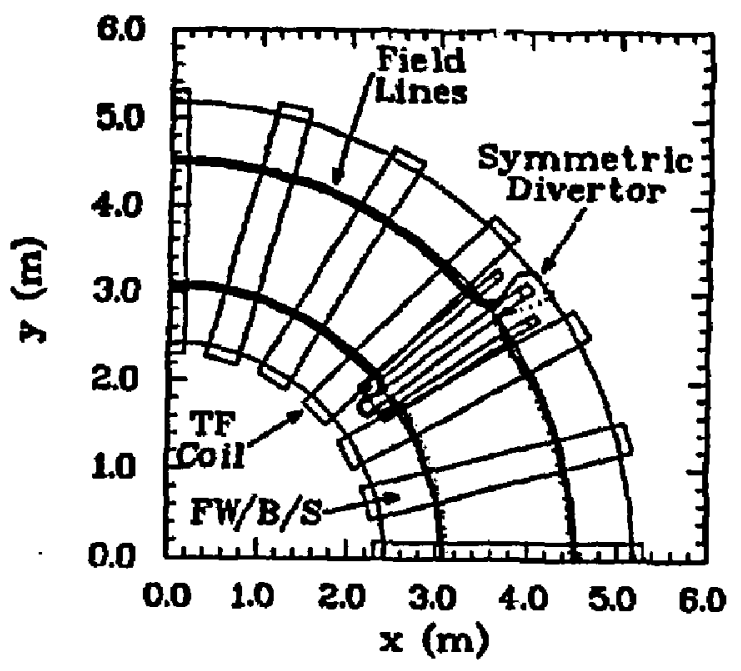

P18. 1.1.4-6.

Th.e tuo-dimensional field-1ine tracings (solid lines) for 4 SDs for $r=0.69,0.705,0.715,0.73$, and $0.75 \mathrm{~m}$. Also shoun are the puncture plots for the three-dinensionsl simuletion of plesma and coile $r=0.68$ and $0.73 \mathrm{a.}, 13$

the baste conflguration with 4 SDs ds shown in Flg. 1.1.4-6; design parameters are glven in Table 1.1.4-2.

The SD Is clearly preferable to the BD on the basis of reduced connection length. The presence of an SD does not signifleantiy perturb the flux surfaces In the outer region of the RPP plesua and, therefore, should not signtefcantily affect confinement. The SD preserves the compactness of the fusion pover core in a a intainable seonetry.

The concentration of heat flux in the closed divertor in the Titan/RFP reactor requires the introduction of heat reduction sechniques. One technique 1. to Introduce impurities into the divertor chaber to radiate the energy being transported long field linus before intercepting the neutralizer plate. Tiling the neutralizer plete relative to the orlentation of the fleld ilnes vill reduce the heat flux. The peak heat flux, which occurs on the fleld lines adjacent to and outside of the separatrix, entering the divertor chamber is typlcally high (189 $\mathrm{wH} / \mathrm{m}^{2}$ ) so that the heat flux id,zident on the neutralizer plate is also high $\left(69.7 \mathrm{nW} / \mathrm{m}^{2}\right)$ for the Titan/RFP. 
The senrch for additional hat-flux-reduction technlques led to the exanination of the open divertor configuration shosn 10 Fig. 1.1.4-7. The difference between the open and closed divertors is the location of the neutralizer plate and the nethod for keeping neutials enitted from the plate out of the plesua, In the closed divertor the plate is hidden from vieu of the plesw with the exheust-intercepting plete face directed away from the plesma to deny an eaitted neutral atraight path to the plasm. The return of neutrals to the plasa is further inhibited by the alniona size of the divertor throat aperture and by viscous entralnent. In the open divertor the plete is located near the null vhere the Eleld 11 nes are spread furthest apart. U1th the place In full vieu of the plasma, the enitted neutrals aust be confined to the scrapeoff layer by positioning the plate no closer than approxiately four lonization

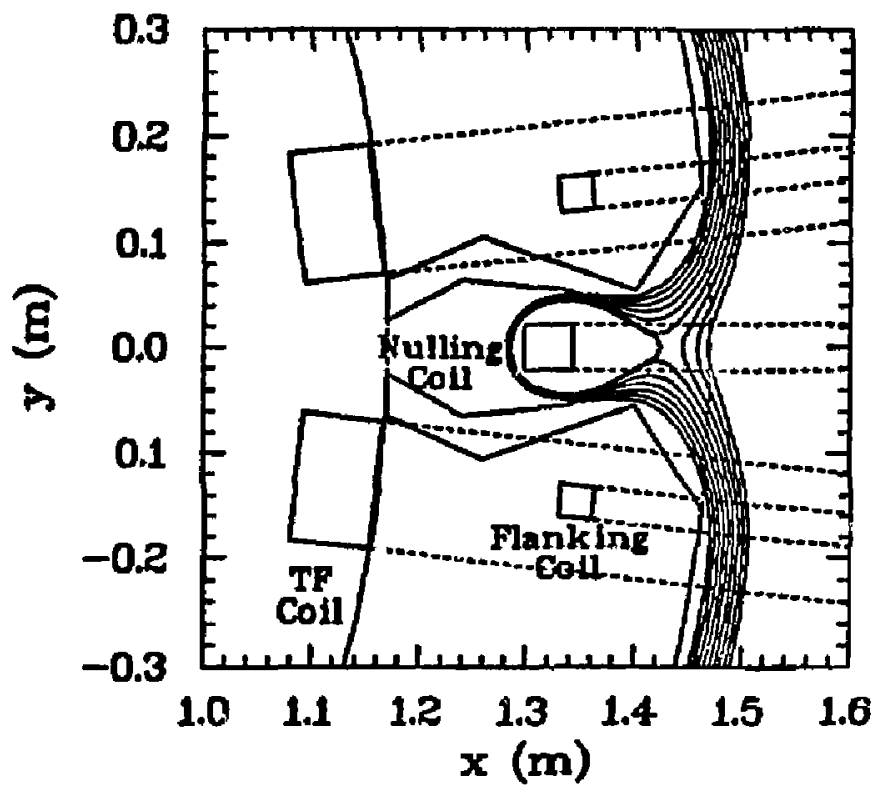

Fig. 1.1.4-7.

Equatorial-plane vieu of the inboard side of an open toroldal-field divertor howing the locetions of the toroldal-field (TF) and divertor colls, the first-vall and divertor surfaces, and the two-dimensional field line tracings about the separatrix. 
mean-free-paths from the separatrix. This mean-free-path constraint on the plate location ensures that an enitted neutral has substantial probability of belng lonized before entering the plasma. The tonized particle then would flov along the field line back to the plate to be reneutralized, but at a location furth-r from the separatrix. This fontzation/reneutralization process valks the exhaust particles way from the separatrix and into the vacuum port. The resulting heat flux $\left(2.42 \mathrm{~kW} / \mathrm{m}^{2}\right)$ incident on the open divertor plate is nearly an order of magnitude lover than for a closed divertor. Consequently, additional efforts on divertor design are focused on the open divertor.

\subsubsection{Current Dr£ye}

Oscillating Fleld Current Drive (OFCD). Steady-state current sustainment by Oscillating Pield Current Drive (OFCD) employs a technique in which the toroidal and pololdal magnetic flelds at the plasma surface are modulated in quadrature at audio frequencles.12,17.33.34 The non-linear coupling of these modulations by the plasma is predicted to provide steady-state maintenance of the mean magnetic fields and hence, the plasma current. Because this technique is technologically simple, non-invasive, and scales well to high temperature, high density plasmas, the posstbillty exists for a relatively simple curzent drive system in future large RFP devices.

One interpretation of the physical mechanism behind OFCD involves the concept of magnetic helicity injection. Magnetic helicity is a measure of the ropological linkage of magnetic lines of force. It has been conjectured that the total magnetic helicjty of plasna configuration is not strongly affected by plasma turbulence and is, in fact, dissipated on a slow resistive time scale. This confecture, with origins in astrophysical research, and plasma physies research, was proposed as an explanation for the self-reversal seen in early RFP experiments. Its primary consequence is that relaxation processes in the RFP lead to unique equilibria in which magnetic energy, dissipated on a fast turbulent time scale, is minimized subject to the constraint of "constant" total magnetic helicity.

Preliminary measurements on the RFP indicate that, within experimental uncertainty, helicity dissipation is indeed controlled by simple crlisional (ahmic) pronesses.4s This demonstration is significant because it indicates how the plasma currents, and hence the confining fields, in an RFP may be sustained. All that is required is a means for replacing the helicity that is lost on the slow resistive time scule (e.g., OFCD), and relaxation processes do the rest. 
An experimental progransi10 is presently undervay on $2 T-40 \mathrm{~K}$, that will test the concept of OFCD. A successful current drive denonstration on $2 \mathrm{~T}-40 \mathrm{M}$ vill be a signiflcant step in proving the OFCD technique. Hovever, because the test will be conducted at relatively lov values of current and temperature, it wil not be definitive with respect to reactor operation. If resistive MHD is the mechanist responstble for the RFP relaxation process, then the results from zT-4OM should scale vell to a reactor. The resistfye HHD time, which sets a lover linit on the modulation perlod to insure that the plasma remains near a relaxed state, scales with the plasma resistivity as $n^{-1 / 2}$. Simulations have shown that the reactor current drive system can operate at high efficiency with flux and current modulations less than $1 \%$, at 25 to $50 \mathrm{~Hz}$ which is well under the inverse resistiv? HD time. Also, penetration of the plasma by an applied oscillating electric fleld should not present a problem since nonlinear tearing modes have been shown to allov this process to occur on anomalously fast time scales. The effect of thase modes on plasma confinement is an open question that is under investigation.

A model of these processes has been developed, and is used for initial evaluations in the TITAN/RFP study.22 The following interin observations and conclustons have been made.

(1) Small plasma current suings ( $\left.\delta I_{\phi} / I_{\phi}=0.02\right)$ and flux sulngs $\left(\delta \phi / \phi_{0}\right)$ vere sufficlent to drive steady-state currents in the $-18-20 \mathrm{MA}$ range with resistive povers in the plasina of - 8-10 MW.

(2) Large power flowed past plasma surface (20-30 $\mathrm{MJ}$ at a frequency $(\omega)$ of $60 \mathrm{~Hz}$ ), but the real pover consumption vas 8-10 $\mathrm{HW}$.

(3) The total/circuic reactive power ratio vas -25 , with a reactive pover of -4 GU flowing past the plasma surface, and a reactive pover of - $170 \mathrm{HH}$ in the external helicity circuit.

(4) A Ilner resistance of $R_{L} \geq 2 \mathrm{mQ}$ ( $\tau_{L}<1.3 \mathrm{~ms}$ ) vas required to hold the power dissipated by the liner to $-8 \mathrm{MW}\left(-80 \mathrm{MW} / \mathrm{m}^{3}\right.$ of liner). The design of an actual "shell" (1.e., liquid-metal cooled blanket, first-vall, shields, suppores, gaps, penetrations; that gives the required $R_{L}$ or $n_{L}$ (liner resistlvity) is a cruclal issue. 
(5) The energy flow across plasna surface (20-30 MJ) vas small, but not negligible when conpared with the plasma kinetic energy $(-10 \%)$. For example, tha basecase conditions specteled a total stored klnetic energy of 115.a MJ. Hovever, the energy flow essociated with ofcD vas small and negligible when compared wth the plasas magnetle energy (1-2X) in that $\beta=B_{\Theta} / 2=0.1$ for a stored nagnetic energy of $1,530 \mathrm{~mJ}$.

Electrostatic Helicity Injection"1. The Taylor minimun energy principle bas been shown to be useful in understanding the wagnetic fleld structure of RFPs. The principle states that an RFP will relax (on a tinescale shorter than the resistive decay tine) to a state of minimum energy while conserving helficity. Helicity decays only on the longer resistive decay time. Thus to sustain an RFP helicity must be supplied to the RFP while relaxation vill malntain the agnetic profiles. Helicity an be supplied either inductively (using transforner) or electrostatically (using electrodes). RFPs bave been sustained through Inductive bethods and Spheromaks have been sustained electrostatically. Some advantages of electrostatic injection are that it is intrinsically steady state with no volt-gec linitutions and it is simple. The disadvantage is that the infector injects plasma as vell as helicity.

The first tests of an electrostatic injector on an RFP have been reported. Unfortunately the injected plasna nasked any effect of the injected helicity. Hovever, a nodel vas verified that predicted the amount of plasma infected and the Inpedance of the injector. The model will be useful in the design of future injectors.

Some of the key issues can be identified as follows:

- Identify the eppropriate divertor'electrode and auxiliarycoll configuration for efficient helicity injection.

- Deternine the fraction of flux to be diverted to the electrode divertor.

- Explore key uncertainties/unknowns such as current density uniformity at the electrodes, helicity injection efficiency, ohnlc dissipation in the edge plasma, edge temperature, electrode arc drops, etc. 
Herging Plasmas. In the Doublet reometry of the Hultipinch Experiment two separate pinches vere formed in one discharge chamber. These tuo pinches later fused into one pinch in which the total toroidal current rewained constant.42 In fact, it was difficult to prevent the uerging. Phillips"3 has proposed an "RFP Current Hultiplter" in which a high current RFP experiment is produced by the accumulation of aeries of small RFPs in an eccusulator. The accumulator, made of lov resistance materlal, vould contain the accumulated RPP. Here the pinch current vould decay by its electrical $\mathrm{L} / \mathrm{R}$ decay rate. If the rate of merging the plasmas exceeds the rate required for sustainment, the plasma is heated. The merging of such plasmas vould contfnue unt1l alpha-particle heating became the dominate heating mechanlsm. The addition of smali RFP pinches would then replace the lost current and agnetle fleld energy periotically to sustain the pinch.

Bootstrap Current Drived2. Neoclassical theory predicts the existence of a "bootstrap current" caused by radial diffusion. The high beta that characterizes the RFP suggest a large bootstrap current, but the high aspect ratso vill tend to reduce the effect. For typical geometrles and conditions a bootstrap current of only $1.6 \mathrm{HA}$ compared to a plasma current of approximately 18 MA would be generated. Significantly steeper pressure gradients and lover aspect ratios vould be requized to make bootstrap current a sigrificant contrbutor, if 1 t exists at all.

Alfven-Vave (AW) Current Drive ${ }^{2}$. Varlous scaling relationships applied to the 17.8 MA TITAN/RFP design, estimate the following requirements for steadystate current drive: $73,115,137$ Mr. Because of the higher aspect ratio in the RFP geometry, problews vhith tend to impair the current drive will be less severe for the RFP compared to the tokawak.

Lovez-Hybrid (LH) Current orived2. The results of applying two tokamak relationships to the $18 \mathrm{MA}$ TITAN/RFP baseline design gives 207 and $286 \mathrm{MW}$. It can be seen that the $L H$ vave is less efficient than the Alfven-wave as applied to the RFP.

Relativistic Election Beams (REB) ${ }^{12}$. Reference 44 presents a detailed design for a $R E B$ current-drive system for the FED-A reactor as vell as calculations indicating that the current-drive pover for such a system can be taken approximately as 


$$
\mathrm{P}^{\mathrm{REB}}-2 \mathrm{I}^{2} \mathrm{R}_{\mathrm{p}}
$$

where $R$ is the plasma resistance, which for the TITAN/RFP baseline gives 16 MU consistent with a $1.6 \mathrm{MJ}$ QEB pulse energy with an interpulse period of $0.1 \mathrm{~s}$. Although the lou-powe: requirenent makes REBs attractive, a number of obstacles to implementation can be identified. Firstly, the REB systems involve the repeated firing of capactior banks of megavolt range, giving rise to questions of reliability when applied to a commercial reactor. Secondly, theory indicates that the REB current will only penetrate a fer centiaeters into the plasma, leading to a hollow current profile and disagreement with the RFP design. Deeper penetrations can be achieved if very high voltages ( $50 \mathrm{HV}$ ) can be used. A combination of REB to provide current in the outer plasma with AW drive to provide current at the plasma core may offer some advantages in power savings, albeit at the cost of additional complexity, One hope in dealing with the hollow current profile is that the REB-created currents will diffuse anomalously rapldiy into the plasma core or be transported into the central plasma through HHD activity.

\subsubsection{Heating}

ohmic. Ignition by ohmic heating is consistent vith classical plasma resistivity and is a strong possiblility in the RFP because of the high current density. Classical plasma resistivity is observed in the experiment ${ }_{+}{ }^{45}$ where the higher voltages required to drive current in RFPs relative to tokamaks being a result of geometric factors (1.e., bigher pitch) rather than "anomalous " resistivity. Ohmlc heating is poverful compared to other heating methods, and the critical 1ssues are related more to transport scaling.11 The regeneration of the toroidal flux via the dynamo effect, wherein the toroidal current generates poloidal current, apparantly dissipates ittlle pover beyond the classical ohmic heating involved. This implies that the OFCD technique for driving steady-state toroidal current may be quite effictent.

For ohmically heated tokamaks and RFPs of similar dimension, a comparison at simflar currents shows that the tokamak achieves a better confisement time and higher temperature, but at a lover value of plasma beta. However, when the comparison is made at the same total magnetic field, the confinement time and temperature are equivalent In the RFP and tokamak experiments.“6 These results, based on a comparison of RFP and tokamak experiments, are in agreement with 
recent theoretical results.47 This theoretical vork also concludes that if ohmically heated RFPs and tokamaks could be compared at - similar plasma temperature, density, and beta, then the RFP confinement would be better than a tokarak. These theoretical results are encouraging, but until verifled by experimerit, do not reduce the present large uncertainty about RFP confinement in the reactor relevant regime. The next generation of multi-megampere RfP experiments (RFX and CPRF/ZT-H) vill be important steps in understanding RFP physics.

A significant advantage of the RFP over the tokamak is that the current density is not linited by stability. To maintain stability in a tokamak, as the current density is increased the toroidal field must also increase to maintain $q>1$. This condition does not apply to the RFP, thus providing another degree of freedow there the ohmic heating input to the plasana can be Increased without significant Increases in the external toroldal field strength. As a result of this feature, ohmic heating to ignition may be possible and auxiliary heating components are probably not required. Ohmic heating results from the naturally occurring distribution of currents vithin the plasma and avoids the problems associated with the introduction and distribution of auxiliary heating power frow locallzed sources.

Experimentally, the RFP is observed to operate at a roughly constant beta over a wide range of operating conditions. Furthermore, the energy containment time 15 observed to scale vith a classical-1ike dependence, at a constant value of I/N. Reactor studies indicate that the ohmic startup (flux requirement) and device size are not impacted unless the energy containment time scales with the current to the $<0.8$ power $^{7}$, which implies a good safety margin. The RFP is an ohmically heated device and as such all of the current drive schemes discussed in the previous section also result in plasma heating.

Alpha-Particle. A discussion of alpha-particle effects on the plasma is given in Sec. 1.1.2. Classically, the alpha-particle energy will be confined in an RFP. The effect of turbulence, however, on the alpha-particle containment has yet to be explored. A transition from a heavily ohmically heated plasma (strong heating in the outer plasma regions) to one that is alpha-particle heated (strong central heating) may impact plasma stability and confinement; hovever, the tendency of the plasma to maintain a "minium energy state" should allow the continuous "relaxation" of the plasma to stable profiles. 


\subsection{SPHEROMAKS}

\subsubsection{Spheromak Characteristics}

The spheromak concept has unique features that distinguish it from other magnetic confinement configurations. Like the RFP, the spheromak has a toroldally symmetric magnetic field geometry in which the toraidal and poloidal components of the field are comparable; and, furthermore, this field and its structure are determined by strong currents floving within the plasma. Unlike the RFP, however, the spheromak possesses comparable scale lengths in both the toroidal and pololdal directions and requires no coils or other structure linking the confined plasma torus. The compact geometry, simple topology and strong Internal currents of the spheromak present many potential reactor advantages. These include simplification of external blanket and coll structures, high engineering $B$ (plasma pressure compared with external field requirements), high power density and reactor mass utilization, natural divertors at the magnetic separatrix, the options of translation or compression of the configuration between formation and burn, and strong ohmic heating which reduces or eliminates the need for heating by auxiliary means.

The spheromak equilibrium is envisaged as a configuration of nested toroldal magnetic flux surfaces generated by plasma currents that nearly satisfy the force-free condition of parallel $\vec{J}$ and $\vec{B}$. Outside 1 ts separatrix, the magnetic pressure of the spheromak equilibrium must be supported by externally driven magnetic fields or (on short time scales) by image currents flowing in nearby material conductors. Some external structure is also required to prevant the gross tilt and shift instabilities that will occur when a spheromak equilibrium is supported by constant external fields alone. For a given separatrix shape, the factors that determine the distribution of internal plasma currents include: the local plasma resistivity, the application of "curtent drive" techniques, and the relaxation processes associated with unstable current-driven (kink) modes. Regarding this last topic, the principle that relaxation leads tovard a state of minimum magnetic energy on time scales for which magnetic helicity is conserved has found wide applicability in spheromak research. 36

Spheromak experiments to date have employed a variety of techniques for Initial formation of the configuration." These methods differ in their use of Inductive colls and electrodes to generate the required plasma currents and linked magnetic fluxes. They have implications for steady-state technology only 
to the extent that they impose restrictions on the methods for spheromak sustainment that may be adopted. Similarly, a varlety of passiye conducting structures, ranging from solid-vall flux conservers to quite open "flgure 8" colls, have been used to provide stability to gross notions on the relatively short time scales of the present experiments. The requirements for such stabilizing structures and the degree of plasma contact that they experience are key issues in spheromak research and of obvious relevance to the technology requirements of a steady-state system.

A recently completed Spheromak reactor study's indicated the possibility of compact, high-power-density fusion reactors with operationally simplified fusion pover plants. A fusion-pover-core (FPC) which stressed single-plece FPC maintenance, steady-state current drive through electrostatic helicity infection, a simplified co-axial electrode divertor, and efficient resistivecoll equilibrium field colls was explored. The optimal FPC size and cost estimates projected a system that competes aggressively with the best offered by alternative energy sources while simplifying considerably the complexity that has generaliy been associated with most approaches to magnetic fusion energy.

\subsubsection{Prospects for Steady-State Operation}

The spherowak is not inherently a steadj-state confinement concept because the internal currents and associated equilibrium fialds decay away on the resistive, magnetic diffusion time scale. Under reactor conditions, 4 this time can be appreciable (from 1 to 10 minutes) so even a pulsed system will display many of the technology issues of continuous operation. Furthermore, true steady-state operation is a realistic goal of spheromak development. Though in principle both the polotdal and toroidal fluxes should be mafntained against resistive decay, the current-driven relaxation processes referred to above provide a mechanism for "flux conversion" from one component to the other as a state of lower total magnetic energy is achieved. Though the impact of this behavior on transport and confinement is still under study, it simplifies the sustainment problem and gives rise to two general approaches to its solution. In the first approach, current-drive techniques developed for other concepts are adapted for the spheromak. Then, for example, if the toroidal current is driven in the vicinity of the magnetic axis, the equilibrium poloidal current is also maintained through the relaxation process. Current-drive techniques that have been considered in this context include neutral beam injection and rf waves launched in the lower-hybrid and electron-cyclotion 
resonant frequency ranges. A second approach to spheromak sustainment is based on the vfey that spheronak decay results from the resistive loss of magnetic helicity (flux linkage) and that, because of the relaxation principle, any rechnique that can continuously supply agnetic helicity can provide a basis for steady-state operation. This approach has been demonstrated through the use of magnetized electrodes on the CTX experiment to sustain spheromaks over several resistive decay times.30 The key issue of hellcity conservation during this process has also been confirmed, $\$ 1$ and a steady-state reactor studys 2 has been based on this option. An alternate method of helicity injection, proposed for inductively generated spheromaks such as the S-1 experiment, Involves the phased AC cycling of externally induced fluxes.53 This technique vould be similar to "F- $\theta$ pumping" presently being evaluated for RFP sustainment and It comes under the general heading of oscillating fleld current drive (OFCD). Finaily, $1 t$ should be noted that experinents conducted at laboratorles outside the US have introduced alternative sustainment strategies that are stlll at an early stage of development. These efforts include nerging spheromaks and the use of rotating magnetic flelds (rotamak concept).

\subsubsection{Spheromak Steady-State Technology Issues}

The technology issues associated vith the development of a long-pulse or steady-state spheronak reactor are in many cases genertc ones. However, as 11lustrated In the above discussion, sone unique issues arise. One of these is the need to assure gross stability of the spheromak equilibrium within the reactor chamber. In all experiments to date, stability has been achleved through the presence of passive conductors that are in near proximity to the separatrix or actually link part of the spheromak flux. Similar neasures are also likely to be required in the reactor case at locations inside the blanket. One study" observes that such stabllizing colls, exposed to neutron fluxes in excess of $20 \mathrm{mw} / \mathrm{m}^{2}$, vould require annual replacement. These colls would be actively driven by feedback control circuit. Their geometry, response time, and current levels cannot be specified on the basis of present experiments. Reactor designst 152 have edge fields at the level of 4 to $5 \mathrm{~T}$ with spheromak radil ranging from 1 to $3 \mathrm{~m}$. Stabilization vill require that a small but significant fraction of this field be supplied by the internal feedback coils.

Spheromak sustalnment Ikewise raises technology 1ssues. If a "standard" method of current drive is chosen, its adaptation to the spheromak case is not expected to introduce new features requiring extensive development beyond that 
needed for other concepts. The technology for sustainment by hellcity injection, hovever, is more spectflc to the spheromak progran. OFCD in spheromaks should have many features in common uth its application to RFPs. The key issue again is hov close the AC driven colls have to be to the burning plasma. The only sustainment aethod that has been demuistrated for significant pulse lengths involves the use of DC currents drawn fron magnetized electrodes. Here the major technological issue is electrode eroston. Erosion must be kept low enough that frequent electrode replacement is not required under steadystate operation. Under such conditions, the introduction of metallic Impurities by the electrodes should be negligible. Low electrode erosion has already been achleved in the field of gas-fed magneto-plasma-dynamic arcs and similar nethods are possible for DC hellcity infection. In the design of reactors based on this approachs there is great uncertainty in the fraction of the cotal plasma current that would be drawn by the helicity source. If the current density can be made uniform over the electrode surfaces, it can be as low as $104 \mathrm{~A} / \mathrm{m}^{2}$.

The topological structure of the spheromak endows it with a natural divertor at its separatrix that has the potential of providing an effective means for impurity control. Exploration of this option is a necessary component of steady-state spheronak development.

The Isslie of vall loading is of course a key one in any reactor study. In the spheromak case, the high-pover-density compact-reactor approach has been chose، because of the various cost advantages that have been clted in other vork.11 Thus a maximum permisstble limit of $20 \mathrm{mu} / \mathrm{m}^{2}$ has bean adopted. The technology required to handle this flux vould not be substantially different between spheromaks and other concepts. Some advantages can be expected in the spheromak case because of the simpler topology of the burn chamber.

\section{REFERENCES}

1. H. A. Bodin and A. A. Nevton, "Reversed-Field Plnch Research", Nucl. Fusion $20,1255,(1980)$.

2. D. A. Baker and V. E. Qulnn, "The Reversed-Field Pinch", Chap. 7 of Fusion, Vol. 1, Part A, E. Teller, Ed., (Academic Press, Inc., Nev York, 1981).

3. D. A. Baker, "The Role of Magnetic Reconnection Phenomena in the ReversedField Pinch," in Magnetic Reconnection in Space and Laboratory Plasmas, Z. U. Hones Jr., Ed., (American Geophysical Union, Washington, D.C., 1984 ). 
4. J. A. Phillips, D. A. Baker, and R. S. Massey, "Developnent of the Reversed-Field Pinch Experimental Program at Los Alamos", Nucl. Fusion 25 . 1321 (1985). Overview papers on RFP programs in the United Kingdon, Japan, and Itsiy appear in the Hagnetic Confinement Sec, 4 (p, 1289) in this same volume,

5. H. H. Malsel, et. al., "The Fusion Pover Research Program at GA Technologies Inc.," Nucl. Fusion 25, 1113 (1985) (OHTE, p. 1121).

6. T. Tamano, "The OatE iteactor Concept", Nucl. Tech./Fustion 3, 89 (1983).

7. C. Copenhaver, R. A. irakowsk1, et. al., "Conpact Reversed-Fleld Reactors (CRFPR)", Los Alamos Mational Labozatory Repoit LA-10500-HS (1985).

8. H. A. Bodin, R. A. Krakowski, O. Ortolan1, "The Reversed-Field Pinch: From Experiment to Reactor," Fusion Technol. 10, 307 (1986).

9. J. D. Callen, et. al., "TPA Plasma Science Report," Untversity of Visconsin Report (Seprember 1986 ).

10. J. N. Downing, "Technical Assessment of Critical Plasma-Materlals Interaction (PHI) and High Heat Flux (HHF) Issues for Alternative Fusion Concepts (AFCs)," Los Alamos National Laboratory Report LA-10635-HS (1986).

11. R. I. Hagenson, R. A. Krakousk1, C. G. Bathke, et, al., "Compact ReversedField Pinch Reactors (CRFPR): Preliminary EngJneering Considerations." Los Alamos National Laboratory Report LA-10200-HS (1984).

12. TITAN Reversed-Pield Pinch Fusion Reactor Study, Interim Report, 1986.

13. C. G. Bathke, R. A. Krakouski, and R. L. Hagenson, "Prospects for Fusion Applications of Reversed-Field Pinches," Proc. 11th Symposium on Pusion Energy, PP. 383-388 (1985).

14. C. G. Bathke, et. al., "Rlasma Engineering Design of a Compact ReversedFleld Pinch Reactor," Proc. 10th Symposium on Fusion Englneering; Pp. 853-860 (1983).

15. C. G. Bathke, R. A. Krakowski, and R. A. Miller, "A Comparision Study of Toroidal-Field Divertors for a Compact Reversed-Field Pinch Reactor," Proc. 6th Topjcal Merting on the Technology of Fusion Energy, Fusion Tech. B, 1616-1621 (1985).

16. P. Thullen and K. F. Schoenberg, Eds., "ZT.H Reversed Field Pinch Experiment", Los Alamos National Laboratory Report LA-UR-84-2602 (1985).

17. K. F. Schoenberg, R. P. Gribble, and D. A. Baker, J. Appl. Phys. 56, 2519 (1984).

18. R. A. Krakorski, R. L. Miller, and R. L. Hagenson, "The Need and Prospects for Improved Fusion Reactors," Los Alamos National Laboratory report LA-UR-86-827, submit ted to J. Fusion Energy (August 8, 1986). 
19. G. A. Vurden, et. al., Bull. Am. Phys. Soc. 30, 1402 (1985).

20. B.A.B. Bodin, et al. 9th International Conference on Plasma Physics and Controlled Nuclear Fusion Research, Paper IAEA-CN-41/H-5 (1982).

21. R. S. Hassey, et. al., Proc. 6th Topical Heeting on the Technology of Fustion Energy, Fusion Tech. 8, 1571 (1985).

22. P. Thullen (Conpiler), rzT-40U: Conceptual Design of 2-HA Reversed-Field Pinch Expucinent," Los Alamos National Laboratory Report LA-UR-B3-1375 (1983).

23. G. A. Wurden, et. al., "Pellet Refueling in the $2 T-40 H$ Reversed-Fjeld Pinch," Los Alamos National Laboratory Report LA-UR-B6-4011 (submitted to Nucl. Fusion).

24. D. Schuresko, Oak Rldge National Laboratory, private communication (1986).

25. J. N. Downing, C. J. Buchenauer, J. C. Ingraham, R. A. Krakousk1, J. A. Phillips, and R. G. Watt, J. Nucl. Mater. $\underline{128}$ and $\underline{129}, 517-523$ (1984).

26. T. E. Cayton, et. al., "Plasma-Surface Interactlons in the 2T-40H ReversedField PInch," 7th Int. Conf, on PSI, May 5-9, 1986, Princeton, NJ (to be published In J. Nucl. Mater.).

27. J. N. Uoming, "A Large Area Pump Limiter Concept," Proc, 11th Symposium on Fusion Energy, pp. $924-927$ (1985).

28. A. A. Newtion and P. G. Noonan, Culbam Laboratory, private communication (1985).

29. K. Ogawa, Y. Maejima, T. Shtmada, Y. Hirano, and Y. Yagt, Nucl. Fusion 25, 1295 (1985).

30. G. Jackson, T. Carlstron, B. Gurwen, et. al., 6th PSI Conference, Nagoya, (1985).

31. J. C. Ingrahan and L. C. Burkhardt, Bull. Am. Phys. Soc. 28, 1098 (1983).

32. P. G. Ueber, Phys. Fluids 28, 3136 (1985).

33. H. K. Bevlr and J. W. Gray, "Relaxation, Plux Consumption and quast Steady State P1nches," Proc. RFP Theory Workshop, April 28 - May 2, 1980, Los Alamos National Laboratory Report LA-B944-C, p. 186 (January 1982).

34. K. F. Schoenberg, et. al., "F- $\theta$ Pumping and Field Modulation Experiments on a Reversed-Field Pinch Discharge," Phys. Fluids 27(3), 548 (1984).

35. J. B. Taylor, "Relaxation of Toroldal Plasma and Generation of Reversed Kagnetic Fleids," Phys. Rev, Lett. 33, 1139 (1979).

36. J. B. Taylor, "Relaxation and Magnetic Reconnection in Plasmas," Rev. Mod. Phys. 58, 741 (1986). 
37. P. G. Ueber, et. al., Proc. 12th European Conf. on Controlled Fusion and Plasma Physics, Budapest, Hungary (1985).

38. D. A. Baker, et, al., 10th Int, Conf. on Plasma physics and controlled Nuclear Fusion Research, Paper IAEA-CN-44/D-II-5 (1984).

39. K. P. Schoenberg, et. a1., 11th Int. Conf. on Plasma Physics and Controlled Nuclear Fusion Research, Paper IAEA-CN-47/D-II-3 (1986), Paper IAEA-CN-44/D-II-2 (1984).

40. K. F. Schaenberg, R. F. Gribble, and D. A. Baker, J. Appl. Phys. 56, 2519 (1984).

41. T. R. Jarboe, Los Alamos National Laboratory, private communication (Jantrary 1987).

42. P.S.C. Lee and R. J. LaHaye, Bull. Am. Phys. Soc. 29, 1331 (1984).

43. J. A. Phillips, Los Alamos National Laboratory, private communication (1986).

44. D. A. Ehst, Y-K M. Peng, P. H. Rutherford, et. -i* "Relativistic Electron Beam Current Drive in FED-A, An Advanced Performance FED Based on Low Safety Factor and Curtent Drive," Oak RIdge National Laboratory Report ORNL/FEDC-B3/1, Sec. 4.5 (August 1983).

45. K. F. Schoenberg, R. W. Moses Jr., and R. L. Hagenson, nplasma Resist1vity in the Progress of a Reversed-Field Dynamo," Phys, Fluids $27(7), 1671$ (1984).

46. J. C. Sprott, "Summary of the 1982 Small Tokamak Users Heeting," University of Visconsin Report No. DOE/ET/5305i-47, (November 1982).

47. J. V. Connor and J. B. Taylor, Phys. Fluids 27, 2681 (1984).

48. H. Yamada, "Reviey of Experimental Spheromak Research and Future Prospects," Fuston Tech. 9, 38 (1986).

49. R. L. Hagenson and R. A. Krakowsk1, "The Spheromak as a Compact Fusion Reactor," Los Alamos National Laboratory report LA-10908-HS (1987).

50. T. Jarboe, et. Al., "Slow Formation and Sustaisment of Spheromaks by a Coaxlal Magnetized Plasma Source," Phys. Rev. Lett. 51, 1 (1983).

51. Cris 1 . Barnes, et. al., "Experimental Determination of tile Conservation of Magnetic Helicity from the Balance Between Source and Spheromak," Phys. Fluids 29, 3415 (1986).

52. R. L. Hagenson and R. A. Krakousk1, "Steady-State Spheromak heactor Studies," Fusion Tech. 8, 1606 (1985).

53. A. C. Janos and M. Yamada, "Inouctive Sustainment of Spheromaks," Fusion Tech. 9, 58 (1986). 


\section{PartD}

Technical Assessment of Critical Issues for Steady State Operation

\section{Devices and Facilities to Address Steady State Issues}

\section{Contributors}

W.B. Gauster 1

D.M. Goebel ${ }^{2}$

C.D. Henning 3

J.F. Lyon 4

A. Miyahara 5

J. Perkins ${ }^{3}$

J. Sheffield ${ }^{4}$

1 Sandia National Laboratory, Albuquerque, NM 87185

2 Institute for Plasma and Fusion Research, University of Califomia, Los Angeles, CA 90024

${ }^{3}$ Lawrence Livermore National Laboratory, Livermore, CA 94550

${ }^{4}$ Oak Ridge National Laboratory, Fusion Energy Division, Oak Ridge TN 37830

5 Institute of Plasma Physics, Nagoya University, Nagoya 464, Japan

D - 1 


\section{Introduction}

In this section, the confinement devices and laboratory facilities that have the capability of addressing steady state issues are presented and discussed. The confinement devices that are planned for the next 5 to 10 years are first reviewed, and the state issues that can be addressed in those machines and the U.S. laboratory facilities are presented. The general design parameters of the Engineering Test Reactor (ETR), based on the Tiber-I study and from several international design studies, is then presented. Two helical devices, ATF and the proposed Large Helical System in Nagoya Japan, which are anticipated to include the abilit; to operate steady state, are described. Finally, the laboratory facilities in the USA that now address steady-state issues, or have the capability to do so in the near future, are reviewed. The issues and types of expeisınents addressed by each machine are identified.

The topics covered are:

1) Planned confinement devices and facilies before ETR that can address Steady State Issues.

2) General overview of the Engineering Test Reactor (ETR/Tiber II) design.

3) Advanced Toroidal Facility (ATF) Steady State (ORNL).

4) Large Helical System (LHS) (IPP Nagoya, Japan).

5) Laboratory Facilities in the USA for addressing Steady State Issues. 


\section{Planned Confinement Devices Before ETR}

As discussed in the section on Plasma Facing Components, experiments need to be done in confinement devices to resolve issues in the areas of plasma operation, impurity control, and materials effects. Fortunately, a range of machines is available on which this work can be done. In the following tables, updated from the Technical Planning Activity Plasma Science Final Report, December 1986, characteristics of these devices are listed, together with the topics most suitable aciuressed in each.

Table 1 lists the large Tokamak facilities operating in the US, Europe, and Japan, and soon to be operating in the US\$R. Of these, JET emphasizes long pulse operation and investigations of the effects of wall materials on plasma performance.

Table 2 shows the parameters for other major Tokamaks. These machines are, in fact, more suitable for systematic experimentation on impurity control and materials issues than the large, expensive devices. Particularly TEXTOR, Tore Supra and ASDEX have strong programs in these areas.

Table 3 shows the features of existing and planned Stellarators. Particularly ATF, with the possibility of steady state operation at high power densities, will be a key device for impurity control issues.

Tables 4 and 5 summarize the confinement maclines and laboratory facilities presented in this part of the technical assessment that can address steady state issues. In the physics area, several of the issues are presently emphasized in programs on existing machines. In the technology field, most of the facilities described in Section V. have some steady state capabilities,

and only need to be directed to address the issues indicated instead of other near term issues. If the near tern issues cannot be avoided, then other or duplicate facilities are needed. 
TABLE 1. Large Tokamak Facilities

Characteristic

TFTR

JET

JT-60

T-15

\begin{tabular}{|c|c|c|c|c|}
\hline Location & USA & EC/UK & Japan & USSR \\
\hline Experimental Start & 1982 & 1983 & 1985 & 1987 \\
\hline Major Radius (m) & 2.5 & 3.0 & 3.0 & 2.4 \\
\hline Minor Radius (m) & 0.85 & 1.25 & 0.95 & 0.70 \\
\hline Elongation & 1.0 & 1.6 & 1.0 & 1.0 \\
\hline Toroidal Field $(T)$ & 5.2 & 3.5 & 4.5 & 5.0 \\
\hline Plasma Current (MA) & 3.0 & 5.0 & 2.7 & 2.3 \\
\hline Auxiliary Heating (MW) & 30 & 40 & 30 & 30 \\
\hline Heating Pulse (s) & 2 & 10 & 5 & $>1$ \\
\hline Heating Methods & $\begin{array}{l}\text { Neutral } \\
\text { Beam (NB) } \\
\text { ICRH }\end{array}$ & $\begin{array}{l}\text { ICRH } \\
\text { NB }\end{array}$ & $\begin{array}{l}\text { NB } \\
\text { LHH } \\
\text { ICRH }\end{array}$ & $\begin{array}{l}\mathrm{ECH} \\
\mathrm{NB}\end{array}$ \\
\hline Working Gas & H,D,DT & H,D,DT & $\mathbf{H}, \mathbf{D}$ & $\mathbf{H}$ \\
\hline Special Features & $\begin{array}{l}\text { Adiabatic } \\
\text { compression } \\
\text { Tangential BN }\end{array}$ & D-Shape & $\begin{array}{l}\text { Outer } \\
\text { divertor }\end{array}$ & $\begin{array}{l}\text { Super- } \\
\text { conducting } \\
\text { coils }\end{array}$ \\
\hline
\end{tabular}

Program Emphasis

Confineme at high $\mathbf{n T}$

DT breakeven
Confinement at high $\beta$

High-power if
Confinement $\mathrm{ECH}$ at high $\mathbf{n T}$

High-power if control 
TABLE 2 Other Major Tokamak Facilities

\begin{tabular}{|c|c|c|c|c|c|c|c|}
\hline Characteristic & DIII-D & PBX-Ua & C-Moda & ASDEX-Ua & Tore-Sa & FTU-Ua & TEXTOR \\
\hline Location & USA & USA & USA & FRG & France & Italy & FRG \\
\hline Experimental Stan & 1986 & 1987 & 1989 & 1989 & 1988 & 1988 & 1984 \\
\hline Major Radius (m) & 1.7 & 1.5 & 0.65 & 1.6 & 2.3 & 0.95 & 1.8 \\
\hline Minor Radius (m) & 0.7 & 0.3 & 0.2 & 0.5 & 0.7 & 0.3 & 0.5 \\
\hline Elongation & 1.8 & 2.2 & 1.8 & 1.4 & 1.0 & 1.0 & 1.0 \\
\hline Toroidal Field (T) & 2.2 & 2.2 & 9.5 & 3.9 & 4.5 & 8.0 & 2.6 \\
\hline $\begin{array}{l}\text { Plasma Current } \\
\text { (MA) }\end{array}$ & 5.0 & 0.6 & 3.7 & 2.0 & 1.7 & 1.6 & 0.5 \\
\hline Currenl Pulse (s) & 10 & 1.5 & 1.0 & 6 & 30 & 1.5 & 5 \\
\hline Heating Methods & $\begin{array}{l}\text { NB } \\
\text { ECH } \\
\text { ICRH }\end{array}$ & NB & $\underset{\text { ECH }}{\text { ICRH }}$ & $\begin{array}{l}\text { NB } \\
\text { ICRH }\end{array}$ & $\begin{array}{l}\text { NB } \\
\text { LHH } \\
\text { ICRH }\end{array}$ & LHH & ICRH \\
\hline Special Feanures & $\begin{array}{l}\text { High } \\
\text { elongation }\end{array}$ & $\begin{array}{l}\text { Bean } \\
\text { shaping }\end{array}$ & Divertor & Divenor & $\begin{array}{l}\text { Super- } \\
\text { conducting }\end{array}$ & & $\begin{array}{l}\text { Pumped } \\
\text { limiler }\end{array}$ \\
\hline Program Emphasis & $\begin{array}{l}\text { Beta } \\
\text { Confinement }\end{array}$ & $\begin{array}{l}\text { Second } \\
\text { stability } \\
\text { regime } \\
\text { Beta }\end{array}$ & $\begin{array}{l}\text { High } \\
\text { field } \\
\text { If }\end{array}$ & $\begin{array}{l}\text { Confine- } \\
\text { ment } \\
\text { Impurities }\end{array}$ & $\begin{array}{l}\text { Long } \\
\text { pulse }\end{array}$ & $\begin{array}{l}\text { High } \\
\text { field } \\
\text { rf } \\
\end{array}$ & $\begin{array}{l}\text { Impirity } \\
\text { control } \\
\text { PMI }\end{array}$ \\
\hline
\end{tabular}

\footnotetext{
a PBX-U - Princeton Beta Experiment Upgrade

C-Mod - High ficld tokamak at MIT

ASDEX-U - Axially Symmetric Divertor Experiment Upgrade

Tore-S - Supcrconducting tokamak at Cadarache, France

FT-U - Frascati Tokamak Upgrade
} 
TABLE 3 PARAMETERS OF STELLARATOR EXPERIMENTS

\begin{tabular}{|c|c|c|c|c|c|c|c|}
\hline Parameter & WVI-A & -AS & He-E & $\begin{array}{c}\text { Proto-Cleo/ } \\
\text { IMS } \\
\end{array}$ & $\begin{array}{l}\text { Auburn } \\
\text { Torsairon } \\
\end{array}$ & HBQM & ATF \\
\hline Laboratory & Garching & Garching & Kyoto & Wisconsin & Aubum & Washington & ORNL \\
\hline Type & Stell. & Mod-Stell. & Heliotron & $\begin{array}{l}\text { Stell/J } \\
\text { Mod-Sicll. }\end{array}$ & Torsation & Linear $\left\{\begin{array}{c}\text { Heliac } \\
l=1\end{array}\right.$ & Torsairon \\
\hline$R(m)(L)$ & 2.00 & 2.00 & 2.20 & 0.40 & 0.58 & $(2.0)$ & 2.10 \\
\hline$\dot{a}(m)$ & 0.10 & 0.20 & 0.20 & 0.04 & 0.10 & $0.05-0.10$ & 0.30 \\
\hline $\mathbf{B}(\mathbf{T})$ & 3.5 & 3.0 & 2.0 & 0.6 & $(0.3)$ & 0.5 & 2.0 \\
\hline$l_{a}$ & $0.2-0.6$ & 0.39 & 2.5 & 0.6 & 0.4 & -1.5 & 0.95 \\
\hline $4 \mathrm{hn}$ & 2.5 & $2 / 5$ & $2 / 19$ & $3 / 7$ & $2 / 10$ & $1 / 7$ & $2 / 12$ \\
\hline$T_{\text {pulse (s) }}$ & 0.3 & 10 & 0.5 & $<0.1$ & $<0.1$ & $40 \mathrm{~ms}$ & $5-(S S)$ \\
\hline$\langle b\rangle(\%)$ & 0.5 & $(\sim 2)$ & 2 & - & 2 & $(-20)$ & $(\gtrsim 8)$ \\
\hline$\tilde{n}\left(m^{-3}\right)$ & $10^{20}$ & $10^{20}$ & $1.4 \times 10^{20}$ & $-10^{18}$ & $-10^{19}$ & $\left(10^{21}\right)$ & $10^{20}$ \\
\hline $\mathrm{T}_{0}(\mathrm{keV})$ & 1 & $\geq 1$ & $\geq 1$ & $<0.1$ & $<0.1$ & $(-0.2)$ & $(\geq 2)$ \\
\hline $\mathrm{n}_{0} \tau_{\mathrm{E}}\left(\mathrm{m}^{-3}-\mathrm{s}\right)$ & $3 \times 10^{18}$ & $-5 \times 10^{18}$ & $-5 \times 10^{18}$ & $\left(-10^{16}\right)$ & & $\left(-10^{16}\right)$ & $5 \times 10^{18}$ \\
\hline$P_{a u x}(M W)$ & -1 & $\sim 3-5$ & $\sim 2$ & -0.1 & -0.1 & & 5 \\
\hline
\end{tabular}


Table IV. Devices and Facilities avaliable to address Physics Issues for Steady State.

\begin{tabular}{|c|c|c|c|}
\hline $\begin{array}{l}\text { PHYSICS } \\
\text { ISSUE }\end{array}$ & 0.5 years & 5.10 years & $10+$ years \\
\hline Current Drive & $c, E, f, i, N$ & $\mathbf{N}$ & \\
\hline Profile Control & $\mathbf{B}, \mathbf{F}, \mathbf{H}, \mathrm{i}, \mathbf{k}, \mathbf{M}, \mathbf{N}$ & $\mathrm{d}, \mathbf{N}$ & \\
\hline Disruption Control & $\mathrm{h}, \mathrm{n}$ & & ETR \\
\hline $\begin{array}{l}\text { Impurity Transport } \\
\text { and Control }\end{array}$ & $\mathbf{A}, \mathbf{B}, \mathrm{c}, \mathrm{h}, \mathrm{i}, \mathbf{L}, \mathrm{n}, 2$ & $d, j$ & NET \\
\hline $\begin{array}{l}\text { Particle and Impurity } \\
\text { Rennoval }\end{array}$ & $A, B, i, L, N$ & $\mathbf{d}, \mathbf{j}, \mathbf{N}$ & TTER \\
\hline$\alpha$-Particle Physics & (h ?) & D & \\
\hline
\end{tabular}

Devices and Facilities
a ATF
b ASDEX-U
d $\mathrm{CIT}$
e CCT
f DII-D
h JET
i JT-60
j LHS
K PBX-U
1 TEXTOR
m TFTR
1 PMTF
2 PISCES
3 TPX
4 HEBF
5 FELIX

c Alcator C-Mod

g ETR/NET/TTER [planned]

II TORE SUPRA

[planned]
Date of Operation

1988

1989

1989

1993

operating

operating

2000

operating

operating

1992

1988

operating

operating

1988

operating

operating

not operating 
Table V. Devices and Facilities avaliable to address Technology Issues for Steady State.

\begin{tabular}{|c|c|c|c|}
\hline $\begin{array}{l}\text { TECHNOLOGY } \\
\text { ISSUE } \\
\end{array}$ & 0.5 years & $5-10$ years & $10+$ years \\
\hline Materials & $1,2,1, *$ & $1,2, *$ & \\
\hline $\begin{array}{l}\text { Heat Transfer and } \\
\text { Thermal Stress }\end{array}$ & $1, a$ & 1 & \\
\hline Neutron Irradiation & 4 & 4 & ETR \\
\hline $\begin{array}{l}\text { Erosion and } \\
\text { Redeposition }\end{array}$ & $2, *$ & $2, *$ & NET \\
\hline Tritium & $3, \mathrm{~m}$ & $3, d$ & ITER \\
\hline Disruption effects & 1,6 & & \\
\hline $\begin{array}{l}\text { Heating and Fueling } \\
\text { Components }\end{array}$ & $\mathbf{N}, *$ & $\mathbf{N}, *$ & \\
\hline $\begin{array}{l}\text { Steady Stare } \\
\text { Diagnostics and Control }\end{array}$ & $h, n, 2, *$ & $\mathrm{j}, \mathrm{n}, 2, *$ & \\
\hline
\end{tabular}

\footnotetext{
notes: - BOLD letters indicate program emphasis,

- * could be addressed to some degree in existing confinement machines

- symbol code for the devices is given in Table IV.
} 


\section{ETR / Tiber II Design and R\&D Requirements}

The Engineering Test Reactor (ETR) is the present name of a machine that is proposed to test the engineering aspects of a tokamak fusion reactor. The device is considered to operate steady state, with a burn time exceeding hundreds of seconds. This technical assessment of critical issues in steady state operation of a tokamak is aimed at a machine such as ETR.

In the U.S.A., the ETR design team is based at LLNL and the tokamak design study is named Tiber-II. In this section, the proposed machine parameters of Tiber-II/ETR are presented. The goal of the ETR studies program is to develop the technical and programatic basis for an innovative and low cost ETR, and to influence a possible intemational conceptual design activity in this area. Tiber-II is a compact, $3 \mathrm{~m}$ radius, steady-state tokamak with ECH/LH current drive and profile control.

The plasma parameters of TIBER-II are given in Table 1. For comparison, the parameteres for five ETR type machines from different international studies are given in Table 2. The possible operational scenarios for Tiber-II are given in Table 3.

The Tiber-II design accomodates altemative current drive option, blanket test modules, and materials testing capabilities. The decision to pursue complete stendy-state operation for Tiber-II is based on the following points:

- A large $\mathrm{OH}$ coil leads to a large tokamak.

- 100 volt-sec corresponds to a $5 \mathrm{~m}$ major radius machine.

- Tiber ( $3 \mathrm{~m}$ major radius) has about 5 volt seconds of $\mathrm{OH}$.

- Pulsed OH drives lead to:

- lower current densities in magnets.

- significant eddy current heat loads.

- larger structures due to fatigue. 
- thermal fatigue of first wall and limiter.

- High fluence nuclear tests resuire steady-state or very high duty factor.

- Thermal fatigue of blanket modules may precede damage due to neutrons.

- Equilibrium testing of nuclear components is necessary for realisitc results.

The R\&D necds for steady-state operation of Tiber-IL/ETR are given in Table 4. The information is broken down into program elements in a R\&D program, with three year milestones and the design goals of the work indicated in the table. 
Table 1. TIBER-II Plasma Parameters (February 1987)

\begin{tabular}{|c|c|c|c|}
\hline & \multirow[b]{2}{*}{ TIBER } & \multicolumn{2}{|c|}{ TIBER-II } \\
\hline & & $\mathrm{NBI}+$ Bootstrap & NBI+LH+Bootstrap \\
\hline Pfusion (MW) & 200 & 260 & 270 \\
\hline PNBI (MW) & 0 & 50.2 & 17.9 \\
\hline$P_{L H}(\mathrm{MW})$ & 40 & 0 & 17.5 \\
\hline $\mathbf{Q}$ & 5 & 5.3 & 7.6 \\
\hline I $\quad(\mathrm{MA})$ & 7.4 & 10 & 10 \\
\hline$<\mathrm{T}_{\mathrm{e}}>(\mathrm{keV})$ & 24 & 15.8 & 19.5 \\
\hline$<n_{e}>\left(10^{20} \mathrm{~m}^{-3}\right)$ & 0.94 & 0.88 & 1.0 \\
\hline$<\beta>\%$ & 8.1 & 6 & 6 \\
\hline $\mathrm{q}(0)$ & 1.0 & 1.0 & 1.0 \\
\hline$q(a)$ & 3.2 & 3.8 & 3.8 \\
\hline$R(m)$ & 2.6 & 3.0 & 3.0 \\
\hline$a(m)$ & 0.72 & 0.83 & 0.83 \\
\hline $\mathbf{k}$ & 1.94 & 2.4 & 2.4 \\
\hline$\delta$ & 0.6 & 0.4 & 0.4 \\
\hline$B_{0}(T)$ & 5.0 & 5.55 & 5.55 \\
\hline$<\Gamma_{\text {wall }}>\left(\mathrm{MW} / \mathrm{m}^{2}\right)$ & 1.0 & 1.1 & 1.1 \\
\hline$\Gamma_{\text {wall }}\left(\mathrm{MW} / \mathrm{m}^{2}\right)$ & 1.5 & 1.7 & 1.7 \\
\hline
\end{tabular}

D - 11 
Table 2. Parameters of five international ETR design studies (February 1987).

\begin{tabular}{|c|c|c|c|c|c|}
\hline & $\begin{array}{c}\text { OTR } \\
\text { (USSR) }\end{array}$ & $\begin{array}{c}\text { FER } \\
\text { (Japan) }\end{array}$ & $\begin{array}{c}\text { TIBER-П } \\
\text { (USA) }\end{array}$ & $\begin{array}{c}\text { NET } \\
\text { (E.C.) }\end{array}$ & $\begin{array}{l}\text { DNTOR } \\
\text { (LAEA) }\end{array}$ \\
\hline Fusion power (MW) & 520 & 297 & 270 & 600 & 570 \\
\hline Major radius (m) & 6.2 & 5.2 & 3.0 & 5.18 & 4.9 \\
\hline Auxiliary pow(r (MW) & 50 & 50 & 35.5 & 50 & 40 \\
\hline$\tau_{\text {bum }}(s)$ & 670 & 2300 & $\begin{array}{l}\text { Steady } \\
\text { State }\end{array}$ & 670 & 200 \\
\hline Average flux $\left(\mathrm{MW} / \mathrm{m}^{2}\right)$ & 1.2 & 0.88 & 1.1 & 1.5 & 1.3 \\
\hline Fluence goal (MW yr/m & 5 & 0.3 & 3 & 0.8 & $0.3-3$ \\
\hline A vailability goal* & $60-7 \%$ & Low & $\leq 30 \%$ & $25 \%$ & $25 \%$ \\
\hline $\begin{array}{l}\text { Tritium consumption* } \\
(\mathrm{kg} / \mathrm{yr})\end{array}$ & 18 & Low & 4.5 & 7.7 & 6.1 \\
\hline
\end{tabular}

* in final phase 
Table 3. Possible operating Scenerios for TIBER-II (September 1986)

\begin{tabular}{|c|c|c|c|c|}
\hline & $\begin{array}{c}I \\
\text { LH ramp-up } \\
+ \text { L/R decay }\end{array}$ & $\begin{array}{l}\quad \text { U } \\
\text { LH romp-up. } \\
\text { OH inductive } \\
\text { flat-lop + } \\
\text { LR decay }\end{array}$ & $\begin{array}{c}\text { III } \\
\text { Current-drive } \\
\text { @ 10\% avail. }\end{array}$ & $\begin{array}{c}\text { IV } \\
\text { Current-drive } \\
@ 30 \% \text { avail. }\end{array}$ \\
\hline Pfusion (MW) & 342 & 342 & 290 & 290 \\
\hline$\Gamma\left(\mathrm{MW} / \mathrm{m}^{2}\right)$ & 2.3 & 2.3 & 2.0 & 2.0 \\
\hline$\tau_{\text {bum }}(s)$ & 302 & 724 & $\begin{array}{l}\text { Steady } \\
\text { State }\end{array}$ & $\begin{array}{l}\text { Steady } \\
\text { State }\end{array}$ \\
\hline $\begin{array}{l}\text { Number of lifetime } \\
\text { cycles }\end{array}$ & $25,000^{\mathrm{a}}$ & $14,000^{\mathrm{a}}$ & $\begin{array}{l}\ll \text { Max. } \\
\text { cycles }\end{array}$ & $\underset{\text { cycles }}{\ll \text { Max. }}$ \\
\hline Peak fluence (MW yr $/ \mathrm{m}^{2}$ ) & 0.55 & 0.73 & 1.9 & 5.8 \\
\hline $\begin{array}{l}\text { Tritium consumption* } \\
(\mathrm{kg} / \mathrm{yr})\end{array}$ & 0.46 & 0.61 & 1.6 & 4.8 \\
\hline Availability & $4.8 \% \mathrm{~b}$ & $6.4 \%^{b}$ & $10 \%$ & $30 \%$ \\
\hline
\end{tabular}

[ Machine operating life 10 years ]

a $50 \%$ of projected cycles to failure.

b Required to complete maximum nimber of cycles in 10 year lifetime. 


\section{VALIDATING R\&D FOR ITER DESIGN}

The time and scope of the ITER Design effort precludes a comprehensive development effort. Instead focus is placed on high-leverage design issues for which a proof-of-principle or other significant milestone can be achieved prior to a construction decision in the carly 1990 's.

Task

3-Year Milestone

I. Technologies that Reduce Cost A. Radiatiun-tolerant, high
curreit density magnets
B. Radiation-tolerant insulators
C. Pulsed ohmic healing coils

II. Technologies for Steady-State
A. ECH for disruption control
B. Negative ion sources
C. Negative ion RF quadropole accelerator
D. Photodetachment neutralizer
E. Magnetic shielding
F. Pellet fueling
G. Alternate plasmoid fueling

$4 \mathrm{KA} / \mathrm{cm}^{2}, 14 \mathrm{~T}, 40 \mathrm{~cm}$ bore coil

Low temperature data base on poiyimides and advanced epoxies

1 meter, $10 \mathrm{~T}$ pulsed coils

\section{Design Goal}

$4 \mathrm{KA} / \mathrm{cm}^{2}, 14 \mathrm{~T}, 10^{19} \mathrm{n} / \mathrm{cm}^{2}$, 4 meter major radius TF coils

$10^{11}$ rads, $400 \mathrm{MPa}$ bearing strength

$2-3$ meler, $14 \mathrm{~T}, 4 \mathrm{KA} / \mathrm{cm}^{2}$, O.H.
$170-500 \mathrm{GHz}, 10-30 \mathrm{MW}$
and $10 \%$ tunable FEL

$245 \mathrm{GHz}, 2 \mathrm{MW}, 10 \%$ tunable oscillator for FEL, ETA II Altemate:

$245 \mathrm{GHz}$ tunable FEM, d.c.

1 Amp at $400 \mathrm{KeV}$ D.C. accelerator

1 Amp at $1 \mathrm{MeV}$

Highly reflective laser cell for 1 Amp

1 meter long, $10 \mathrm{~cm}$ demonstration

$5-10 \mathrm{~km} / \mathrm{sec}$

$750 \mathrm{~km} / \mathrm{sec}$ with 1 milligram

\section{Amps at $500 \mathrm{KeV}$}

10 Amps at 2-3 MeV

$90 \%$ efficiency $\mathrm{D}^{-} \rightarrow \mathrm{D}^{0}$

30 meters long, 3 meter diameter. less than 1 gauss

$50 \mathrm{~km} / \mathrm{sec}$

$750 \mathrm{~km} / \mathrm{sec}, 5 \mathrm{mg}$ and $1 \mathrm{~Hz}$ 
III. Reliability

A. Carbon/carbon fiber composites for divertor

B. Altemate divertor materials materials

C. Stress corrosion of steels in waler

D. RF components

E. Properties of redeposited divertor materials
$10^{21} \mathrm{n} / \mathrm{cm}^{2}$ with less than $5 \%$

swelling and low erosion

Beryllium/tungsten data base and tokamak experimental evidence of H-mode

$200 \mathrm{MPa}$ and $10^{8}$ second life

Antenna and insulator tests in ionizing radiation

Physical/mechanical properties of C, $\mathrm{W}, \mathrm{Be}$ neutron radialion effects

Data base for joining of strengthened alloys, low temp irradiation effects, tritium permeation and retention

Compatibility with breeder/coolants, mechanical integrity, and multiplication

Demonstrate 1 meter diameler valve and remote vacuum seal

Metallurgical modification of auslenitic stainless steel to remove niobiurn and moly

B. Shielding effecliveness

\section{Streaming experiments correlated with} design
$10^{22} \mathrm{n} / \mathrm{cm}^{2}$ with low swelling and low tritium retention

low sputtering and erosion with redsposition

$600 \mathrm{MPa}$ and $10^{9}$ second life

$20 \mathrm{MW} \mathrm{LH}$ antenna in plasma environment

\section{Net erosion rexes of I criz/year}

$3 \mathrm{MW} / \mathrm{m}^{2}$ heat flux with less than 10 gms of tritium retention and 10 curries/day permeation

$10^{8}$ second life al $2 \mathrm{MW}-\mathrm{yr} / \mathrm{m}^{2}$ with negligible corrosion

Remole sealing of inner plasma chamber

Meets 10 CFR61 class C, withstands 60 dpa

$10^{19} \mathrm{n} / \mathrm{cm}^{2}$ at magnets in $10^{8} \mathrm{sec}$, and less than 200 millirads/hr outside machine 


\section{ATF STEADY STATE}

The Advanced Toroidal Facility (ATF) 1 , illustrated in Figs. 1a, 1b, and 1c, is in the final phase of construction at the Oak Ridge National Laboratory (ORNL). The main parameters are listed in Tabie 1. Rather than limit ATF to a pulse length of $\sim 10 \mathrm{~s}$ (the resistive diffusion time), we have made it capable of operating in true steady state at the $1 \mathrm{~T}$ level in order to accomplish not only the long pulse physics goals but also to permit component development for later phases of the toroidal program.

The predicted performance of the ATF is shown in Fig. 2, where the density-averaged temperature $<\mathrm{T}>$ is plotted for different values of heating power versus volume-averaged density $<n>$. The computer predictions have been benchmarked against data from the successful Heliotron- $E^{2}$ and W VII-A devices ${ }^{3}$. The high beta capability is expected as a result of ATF's direct access to the second stability regime. The high ion temperatures are predicted as a result of the improved confinement coming from the ambipolar electric field.

\section{1) ATF Schedule}

The main factors bearing upon the schedule to operate ATF in steady state are:

- The need to run ATF in the pulsed mode for sufficient time to complete the pulsed mode mission in the beta (MHD) and transport areas.

- The need to run ATF for long enough in the pulsed mode to prepare for steady state operation; e.g., to establish the power deposition pattern on the walls and targets/limiters, to develop the divertors/limiters and to develop the $\mathrm{ICH}$ launchers.

- The need for time to design, construct, and install the new steady state components.

Preliminary analysis of the schedule suggests that first steady state operation at the $400-800$ kW level could begin in 1991 with incremental funding of about 2 MS per year. Operation at the 2-4 MW level would follow in 1992-1993, with the schedule being dependent on the funding rate 


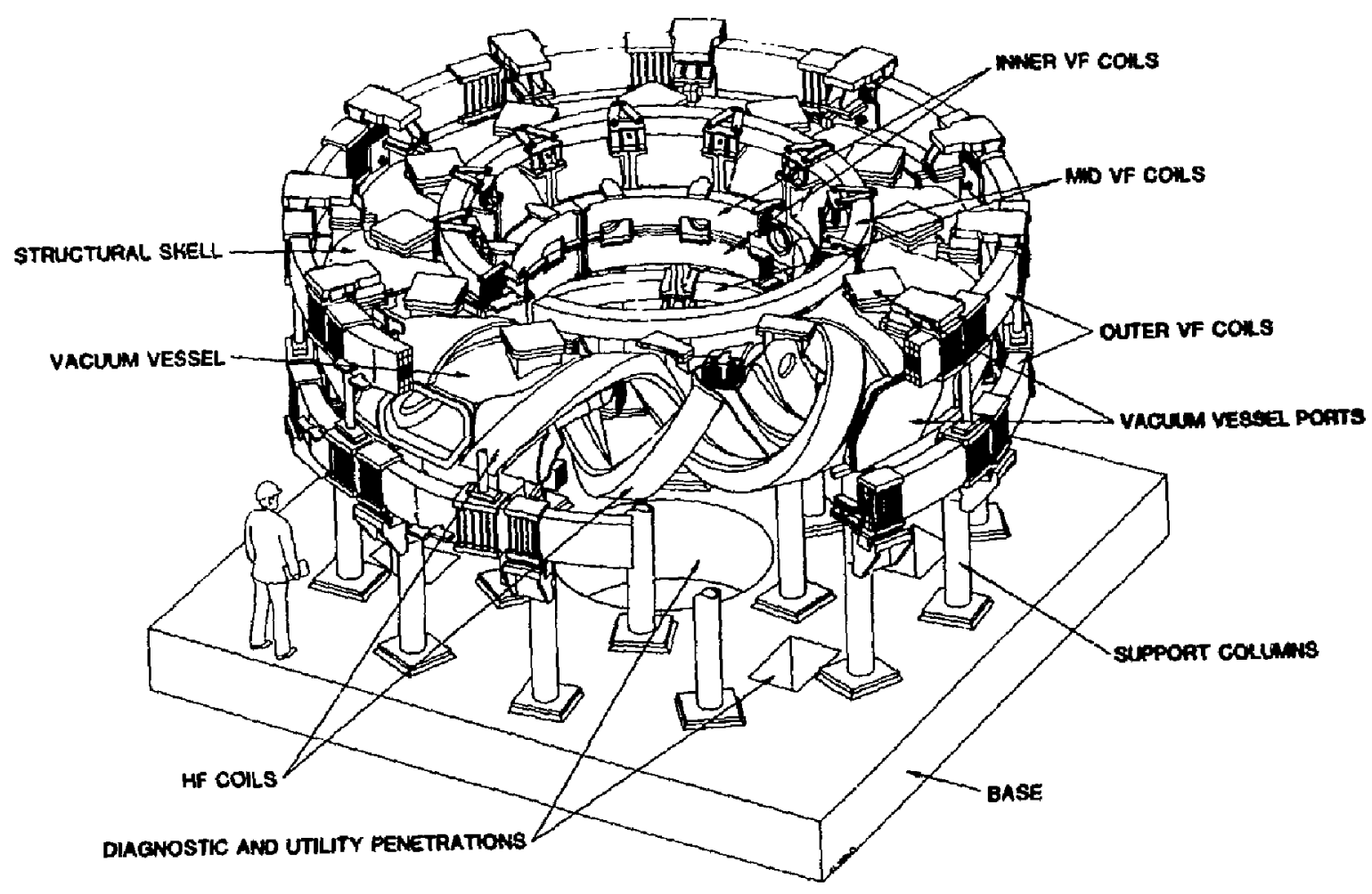


ORNL-DWG B5-3657 FEO

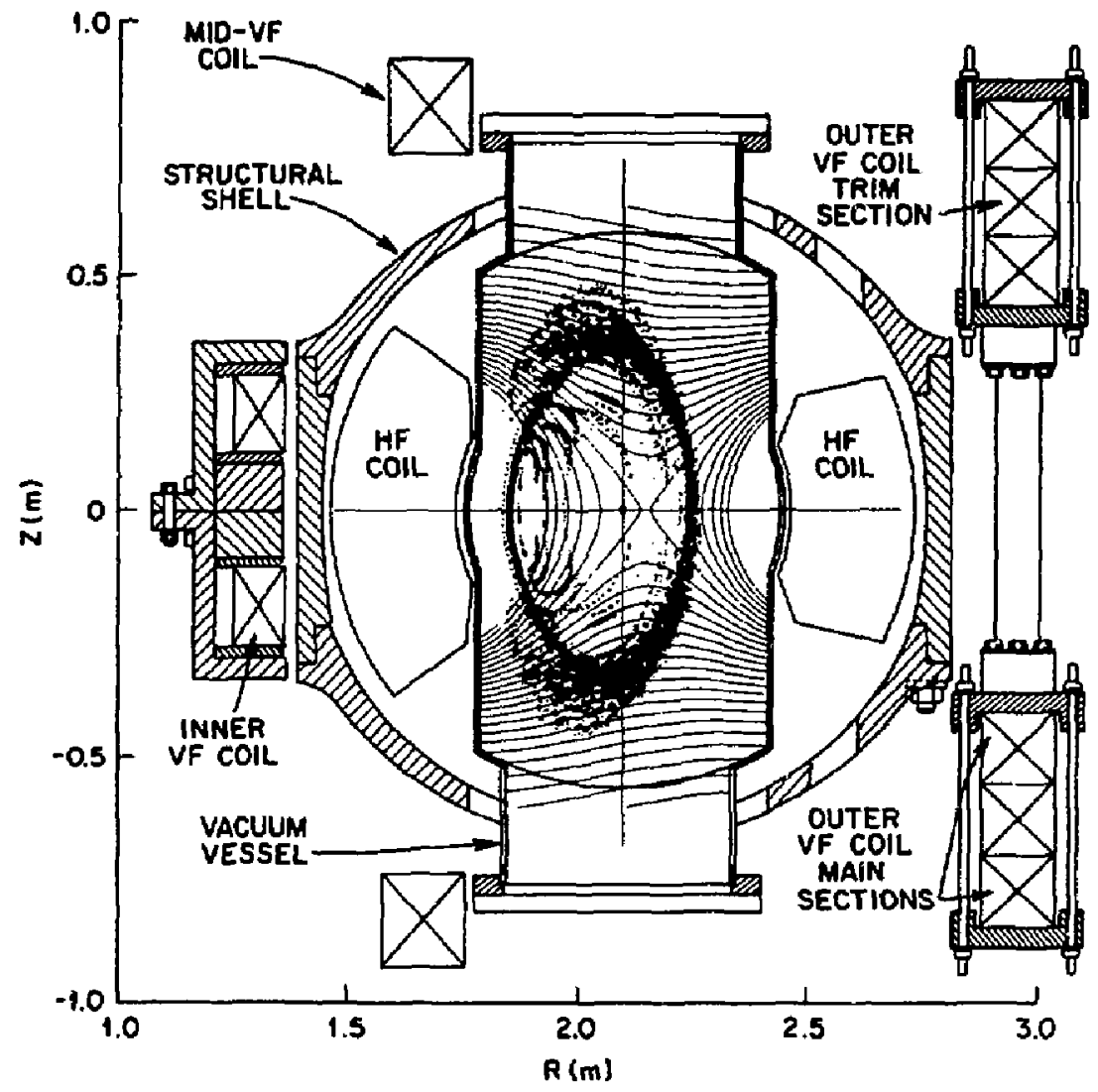

Finure 1b 
ORNL-DWG 85-3656A FED

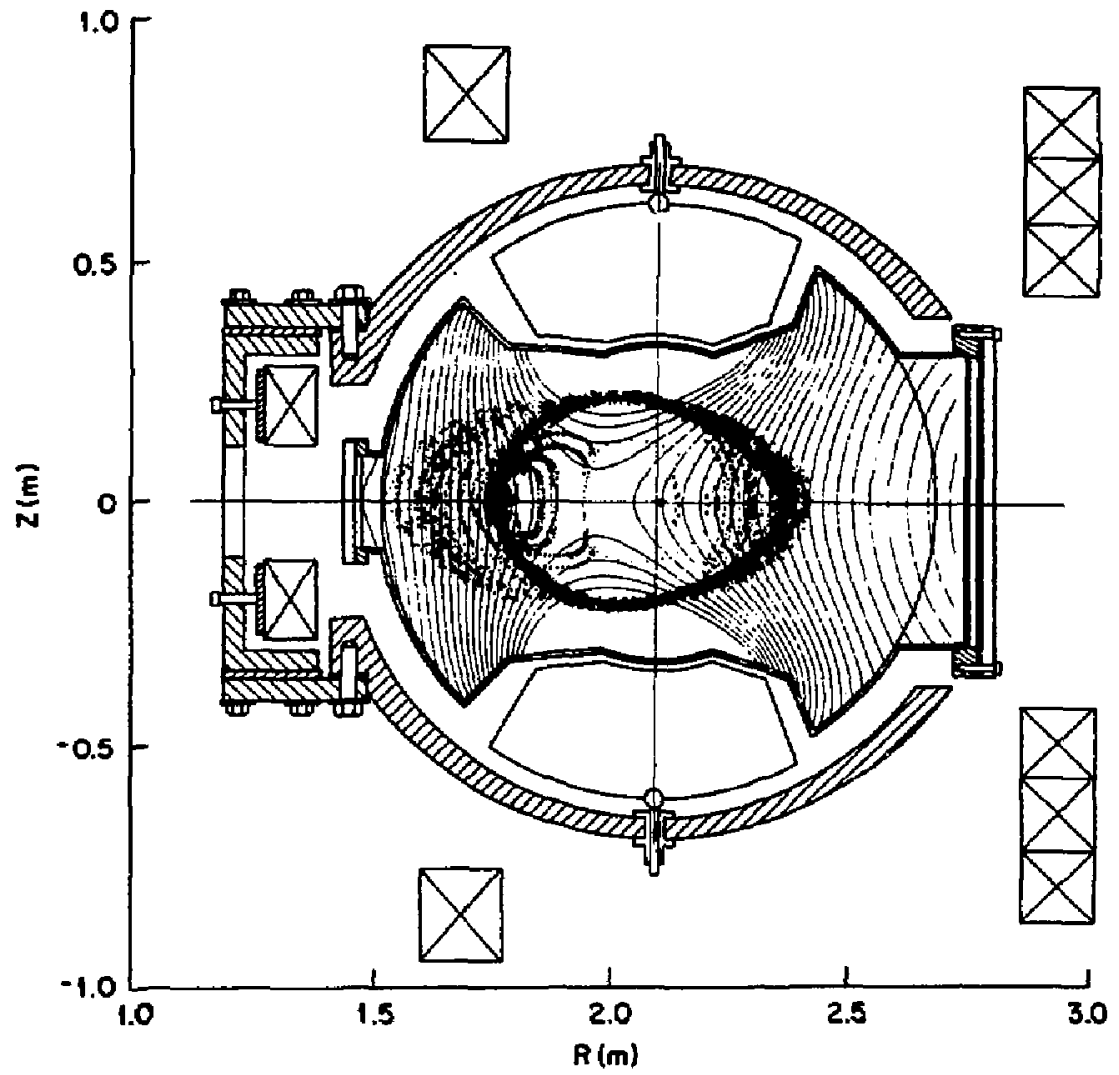

Figure ic 
Table 1. ATF Device Parameters

\section{Size}

Major radius

$2.1 \mathrm{~m}$

Average plasma radius

$0.3 \mathrm{~m}$

Average HF coil radius

$0.46 \mathrm{~m}$

Vacuum vessel radius (inside)

$0.58 \mathrm{~m}$

Structural shell radius (inside)

$0.66 \mathrm{~m}$

Plasma volume

$3.7 \mathrm{~m}^{3}$

Vacuum vessel volume

$10.5 \mathrm{~m}^{3}$

Vacuum vessel interior

$\approx 1.16$ by $0.65 \mathrm{~m}$

Plasma-wall separation

0.05-0.2 m

Field

Magneric field on axis

2 T, 5-s pulse

$1 \mathrm{~T}$, continuous

Initial Plasma Heating

$40-$ to $50-\mathrm{keV}^{\circ} \mathrm{NBI}$

$3 \times 1-1.5 \mathrm{MW}, 0.3 \mathrm{~s}$

53.2-GHz ECH

$2 \times 0.2 \mathrm{MW}$, continuous

5- to $30 \cdot \mathrm{MHz} \mathrm{ICH}$

$0.3 \mathrm{MW}$, continuous

5- to $20-\mathrm{MHz} \mathrm{ICH}$

$2 \mathrm{MW}$, continuous

D - 20 


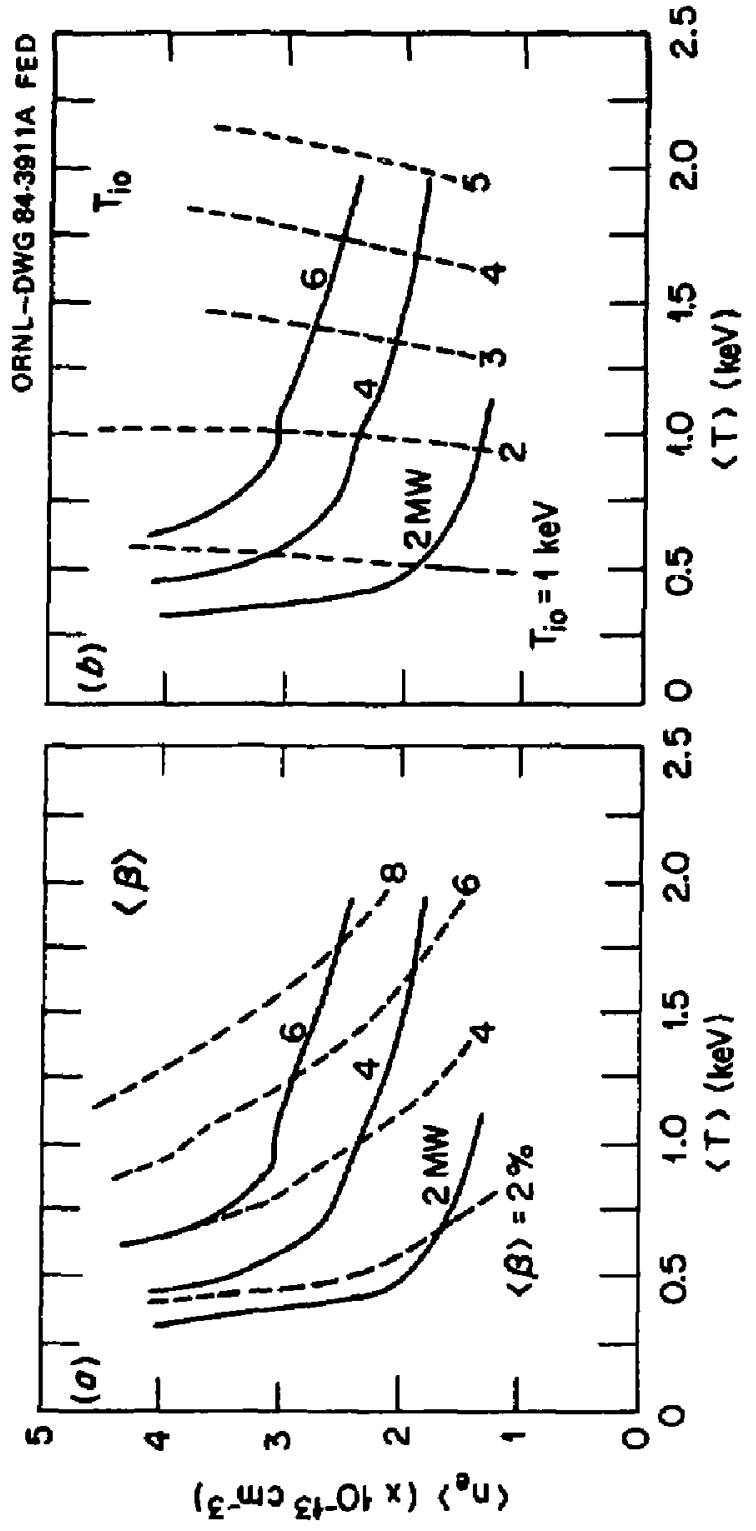


in earlier years. However, useful development work will be undertaken in the earlier years of operation 1988-90. The ATF schedule is summarized in the development plan in Table 2.

\section{2) Steady State Program}

There have been a few previous steady state devices (mirrors in the 1960's, some small stellarators, and EBT at ORNL), but these devices had plasma energy densities substantially smaller than expected in ATF and future, larger stellarators. Moreover, these devices showed differences from pulsed devices; e.g., in EBT it took many minutes for the plasma to reach equilibrium with the vacuum vessel walls. There are, of course, many issues to be faced in running steady state at high power density. The main physics and technology areas that will be addressed in ATF-Steady State include:

- Optimization of the plasma properties--profile, current relaxation, radial potential, shape, etc.--to maximize confinement and beta and minimize impurity problems.

- Study of the evolution of the MHD behavior of high beta plasma for times long compared to the skin diffusion time.

- Investigate plasma-wall equilibration in which $100 \%$ of the hydrogen flux to the wall is recycled unlike present pulsed operation in which the wall may act as a pump during a discharge.

- Develop a steady-state impurity control system.

- Develop a steady-state density control system, which requires the development of steady-state pellet injection and steady-state pumping with hydrogen recycle.

- Apply and develop materials to handle the heat loads with techniques to minimize erosion problems. This activity requires a cooled first wall and steady-state targets/limiters, ICH launchers.

- Develop steady-state efficient Ion Cyclotron Heating (ICH) systems.

Steady-state operation of ATF allows high fluence testing of components, which will make possible rapid tests of erosion properties. For example, 15 minutes of operation in ATF would 
Planned Development of ATF Program

\begin{tabular}{|c|c|c|c|c|c|}
\hline \multirow{2}{*}{ Capsbillty } & 1088 & & 1080 & & 1001 \\
\hline & \multicolumn{4}{|c|}{ PULSED OPERATION } & STUADY-STATE \\
\hline $\begin{array}{l}\text { Magnetic } \\
\text { feld }\end{array}$ & $\begin{array}{l}\text { 2T for } 5: \\
(1 \mathrm{~T} \text { for } 20 \mathrm{~s})\end{array}$ & & & & IT nicady alate \\
\hline $\begin{array}{l}\text { Magnelic } \\
\text { configuration }\end{array}$ & Full obaping & & Belleal axis & 尝 & $\begin{array}{l}\text { Standurd } \\
\text { configuration }\end{array}$ \\
\hline $\begin{array}{l}\text { 10.5V NBI } \\
0.3 \text {. }\end{array}$ & $2-3 \overline{M W}$ & $3-4.5 \mathrm{MW}$ & & 兽 & $\begin{array}{l}3-1.6 \mathrm{WW} \\
0.3 .\end{array}$ \\
\hline $\begin{array}{l}\mathrm{CW} \text { ECH } \\
53.2 \mathrm{GH}\end{array}$ & $0.2 \mathrm{MW}$ & $\begin{array}{l}\text { 0.4 MW, mode } \\
\text { conlrol }\end{array}$ & & 曼 & $\begin{array}{l}0.4 \mathrm{MW} \\
\mathrm{cW}\end{array}$ \\
\hline $\begin{array}{l}\text { lod cyclotron } \\
\text { Henting } \mathrm{cw}\end{array}$ & $\begin{array}{l}6-30 \mathrm{MH} \\
0.3 \mathrm{MW}\end{array}$ & 7 & $\begin{array}{l}\text { 5-20 NB: } \\
2 \mathrm{MW}\end{array}$ & 8 & $4-6 \pi W$ \\
\hline $\begin{array}{l}\text { Vacuum vesed } \\
\text { cooling }\end{array}$ & $\begin{array}{l}\text { 1.5 MJ every } 8 \text { min } \\
150 \mathrm{MJ} / \text { day }\end{array}$ & $\begin{array}{l}\text { B hW } \\
\text { average power }\end{array}$ & & 是 & $\begin{array}{l}\text { 3-5 MW } \\
\text { averse power }\end{array}$ \\
\hline $\begin{array}{l}\text { Particle } \\
\text { control }\end{array}$ & Limikere & $\begin{array}{l}\text { Pump limiler } \\
\text { Pellet injection }\end{array}$ & & 高 & $\begin{array}{ll}\text { cin pump } & \text { Muldplopump } \\
\text { limilex/divestor } & \text { limileso/divertore }\end{array}$ \\
\hline Dingnontics & $\begin{array}{l}\text { Basic physic: } \\
\text { set }\end{array}$ & $\begin{array}{l}\text { Advanced } \\
\text { diegnoatics } \\
\text { Heary ios } \\
\text { beam probe }\end{array}$ & & 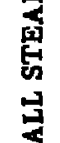 & $\begin{array}{l}\text { Stendy-thale } \\
\text { diegaostica }\end{array}$ \\
\hline $\begin{array}{l}\text { Phyaica } \\
\text { program }\end{array}$ & $\begin{array}{l}\text { Configuration } \\
\text { High beta } \\
\text { Transport } \\
\text { Heating } \\
\text { Particle control }\end{array}$ & $\begin{array}{l}\text { Pulsed } \\
\text { operation }\end{array}$ & $\begin{array}{l}\text { Development } \\
\text { of ateady- } \\
\text { state } \\
\text { approaches }\end{array}$ & 易 & $\begin{array}{l}\text { Extenvion to } \\
\text { ateady abte with } \\
\text { increading power }\end{array}$ \\
\hline
\end{tabular}


subject the limiters to the same paricle fluence as took 3 months to accumulate in the ISX-B beryllium limiter test for $\mathrm{JET}^{4}$. This mode of operation allows the study of material redeposition, of inhibition of impurity accumulation, of methods to minimize wall erosion, of the survivability of thin-cooled coatings, of fuel removal and recycling, of hydrogen retention in the walls, etc. Many of the components required to allow ATF to operate steady state are being completed in the existing program. The development of other components, e.g., pumped limiters and $\mathrm{ICH}$ launchers, will be undertaken as a natural part of the present pulsed program. Work has already begun in most areas, either as part of the ATF program or through the ORNL role in the development and application of plasma technologies. In the pumped limiter area the ORNL program which started on ISX-B has continued through the TEXTOR 5 and Tore-Supra programs ${ }^{6}$, and in the stellarator area through collaboration on Heliotron- $E^{7}$, and with the University of Wisconsin stellarator program. ORNL is responsible for ICRF technology component development and has developed improved ICH components, which are already being used on TEXTOR ${ }^{8}$ and DIII-D. A prototype launcher for ATF has been designed, and a $4 \mathrm{MW}$ ICH antenna is being fabricated for TFTR and TORE SUPRA. In the steady-state area we will benefit from the previous experience with ICH on EBT. The steady-state EBT diagnostics and data acquisition systems, which are designed for plasma conditions similar to those expected in the ATF edge region, will be available.

\section{3) Additions to Operate ATF at $400-800 \mathrm{~kW}$}

The key additional components required for steady-state operation at the $400-800 \mathrm{~kW}$ level are: cooled panels in the vacuum vessel + a first stage pumped limiter or divertor + steady-state pumping; steady-state power supplies for poloidal coils + improved cooling on buswork for all coils + full connection of the cooling water, improved diagnostics and data acquisition systems. The most critical element is the cooled panels inside the vacuum vessel. The uncooled vacuum vessel only allows $1.5 \mathrm{MJ}$ every 5 minutes ( $5 \mathrm{~kW}$ average heat load) for routine operation, or 
$150 \mathrm{MJ}$ per day, before the vacuum vessel heats to $150^{\circ} \mathrm{C}$ and must cool down for up to a few days.

The basis for the cooled panel concept proposed is the development work conducted for the EBT-P vacuum liner. An extensive parameter study along with a detailed development program has established the basic characteristics of this concept (see Fig. 3 and Table 3). It is planned to consult the materials community in choosing the materials for the panels.

\section{4) Additions to Operate ATF at 2-4 MW}

For operation at the 2-4 MW level more ICH power will be used. It could utilize BBC or modified FMIT supplies (see Table 4). Ideally, some of the launchers could be modified and installed during the pulsed operating phase, since they are required to realize ATF full performance capabilities, even in the pulsed mode. An assessment of additional diagnostics and data acquisition systems, beyond those which would be installed as part of the base program, has not been made.

There are three different ICRF heating schemes which will be explored during the initial, pulsed phase of ATF operation as a part of the ATF base program: fast wave heating, slow wave heating, and ion-Bernstein wave heating, each covering a different frequency range. Following an evaluation of the efficiency and advantages of each of these methods, one will be selected for the steddy-state project. The selected option will, in all likelihood, be fast wave heating, the preferred method for tokamaks and for Heliotron-E. However, the other methods will be studied as backup candidates, in case insurmountable difficulties arise with fast wave heating, such as excess impurity influx during the rf heating.

\section{5) Power and Particle Handling}

A more extensive array of pumped limiters or divertors would be required with increased pumping capability. 

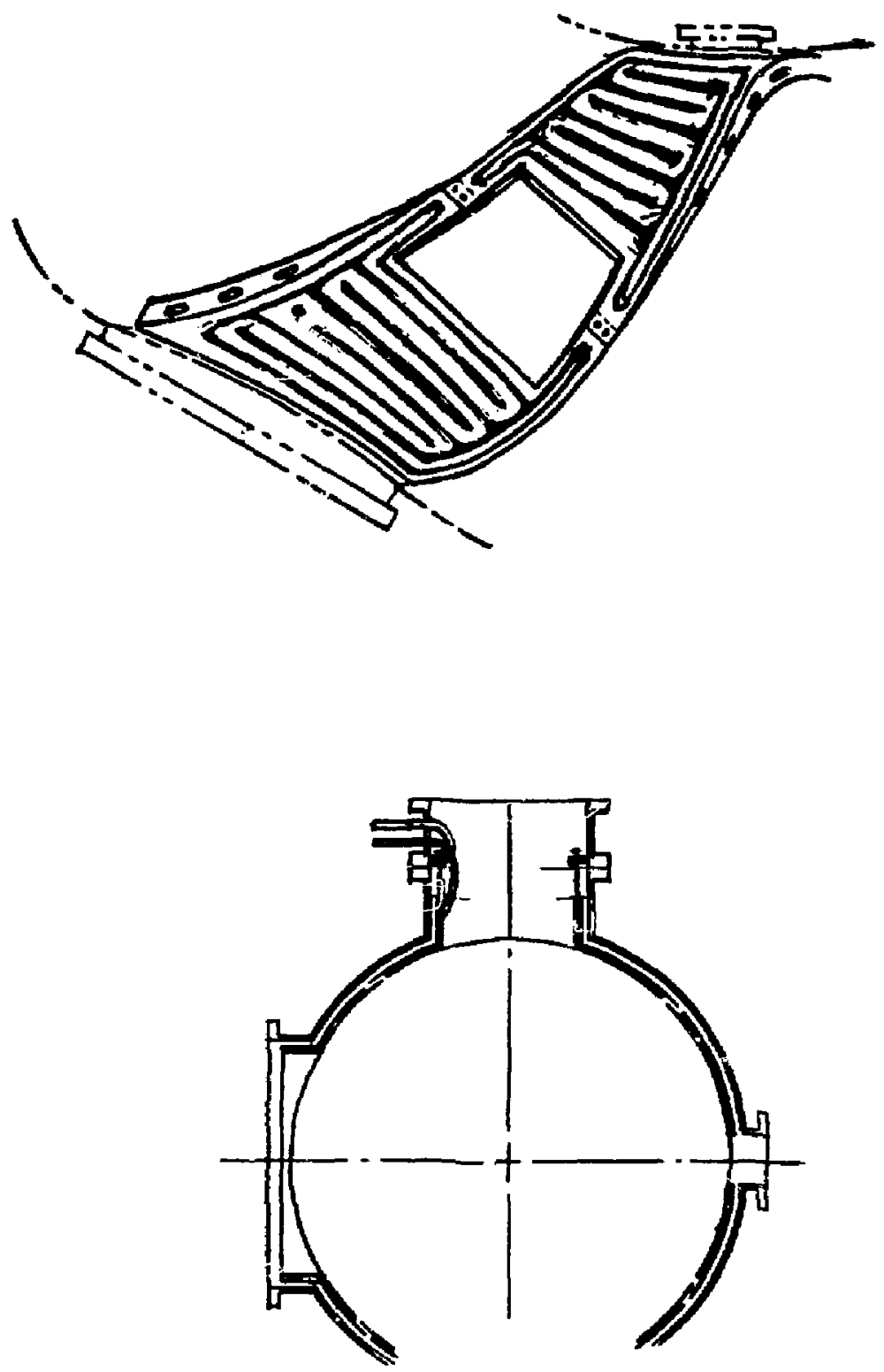

CCOLING PANEL CONCEPT

D-26 
Table 3. Comparison of Panel Characteristics

EBT-P

Peak Flux $\left(W / \mathrm{cm}^{2}\right)$

Average Flux (W/ $\left(\mathrm{cm}^{2}\right)$

L.and Width $(\mathrm{cm})$

F qnt Plate Thickness (cm)

Rear Plate Thickness (cm)

Panel Depth (cm)
20.0

13.5

0.813

0.254

0.127

1.27

ATF-SS

20.0

$10 . n$

0.953

191.0

0.127

1.906

Table 4. Existing CW RF Power Sources Available for Use on ATF

Type Desiznation

AR-1000LM9

AN/FRT-85

81óR-2S

AN/FRT-86 (\#1)

AN/FRT-86 (\#2)

FMrT-Modified'

BBC-Modified ${ }^{2}$
Erequency

$0.2 \cdot 200 \mathrm{MHz}$

$2-30 \mathrm{MHz}$

$30-60 \mathrm{MHz}$

$5-30 \mathrm{MHz}$

5. $16 \mathrm{MHz}$

$40.80 \mathrm{MHz}$

$5-20 \mathrm{MHz}$
Power

$1.5 \mathrm{~kW}$

$20 \mathrm{~kW}$

$20 \mathrm{~kW}$

$100 \mathrm{~kW}$

$200 \mathrm{~kW}$

$1500 \mathrm{~kW}$

$2000 \mathrm{~kW}$

Notes:

1. Thirty second cayability only (power supply limited), time-shared vrith RFTF.

2. Upgraded to $30 \mathrm{MHz}$. 
Steady-state or long-pulse operation of ATF will require limiter or divertor structures capable of absorbing 1-3 MW of heat continuously, and a pumping system to exhaust $\sim 10$ Torr $1 / \mathrm{s}$ of hydrogen. The plasma edge in ATF has a complicated magnetic structure. Diventor scoping experiments are planned for an early phase of the ATF operation to establish the necessary data base for the design and construction of a divertor with the required paricle and power handling capabilities.

\section{References}

1. J. Sheffield, et.al."ORNL Confinement FTP Review", Deparment of Energy, April 1986.

2. K. Uo, et.al., in Plasma Physics and Controlled Fusion Research 1982, Proc. 9th Intl. Conf. Baltimore, 1982 Vol. 2 (IAEA Vienna, 1983) 209.

3. G. Greiger, et.al., Nucl. Fusion, 25 (1985) 1231.

4. P.K. Mioduszewski, et.al., Nucl. Fusion, 26 (1986) 1171.

5. A.E. Pontau, et.al., J. Nucl. Mat., 128\&129 (1984) 434.

6. P. Deschamps, et.al., J. Nucl. Mat., $128 \& 129$ (1984) 38.

7. D.L. Hillis, et.al., J. Nucl. Mat., 145-147 (1987) 496.

8. A.M. Messiaen,, et.al., Plasma Physics and Cont. Fusion, 28 (1986) 71. 


\section{Large Helical System (LHS)}

The Institute of Plasma Physics at the University of Nagoya, Japan, is presently in the proposal and planning phase for a large helical device to be constructed in the 1990's at the new research site outside of Nagoya. The CHS, as proposed, will be the largest helical confinement device yet buile, and tentatively is planned to investigate plasma performance and steady-state operation issues. The figure of merit for fusion, $n_{c} \tau_{E} T_{i}$, versus stored energy is plotted in Fig. 1, showing the anticipated LHS performance in comparison with other confinement machines. The general layout of the machine is shown in Fig. 2. The machine is planned to operate long pulsed $(10 \mathrm{sec})$ to steady state in hydrogen and deuterium. The auxiliary heating for the device will be provided by ECRH, ICRH, and NBI systems, illustrated in Fig. 3. The present design of the helical device features a built in divertor chamber, shown in Fig. 4 , to control the plasma recycling and impurity production location.

While the design is not finalized, the present machine parameter being considered are:

Major radius

Minor radius

Magnetic field

Energy confinement time

Ion temperature

Shape

Average Beta

Wall heat flux

Auxiliary heating
$R=4$ to $5 \mathrm{~m}$

$\mathrm{a}=0.5$ to $0.6 \mathrm{~m}$

$\mathrm{B}_{\mathrm{T}}=4 \mathrm{~T}$

$\tau_{E}=0.1$ to $0.3 \mathrm{sec}$

$T_{i}=3$ to $4 \mathrm{keV}$ at a plasma density of $10^{14} \mathrm{~cm}^{-3}$

$T_{i}=10 \mathrm{keV}$ at a plasma density of $2 \times 10^{13} \mathrm{~cm}^{-3}$

$\mathrm{l}=2, \mathrm{~m}=10$ to 14

$\langle\beta\rangle=4$ to $8 \%$ at $\mathrm{BT}=\mathrm{IT}$

$\langle\beta\rangle=1$ to $2 \%$ at $\mathrm{BT}=4 \mathrm{~T}$

$\tau_{\text {iwall }}=0.8$ to $1.6 \mathrm{MW} / \mathrm{m}^{2}$

15 to $20 \mathrm{MW}, 0.5 \mathrm{sec}$ to continuous

ECRH -2 to $5 \mathrm{MW}$ at 100 to $200 \mathrm{GHz}$

NBI $\quad-16 \mathrm{MW}$ at 100 to $150 \mathrm{keV}$

ICRH - Planned 


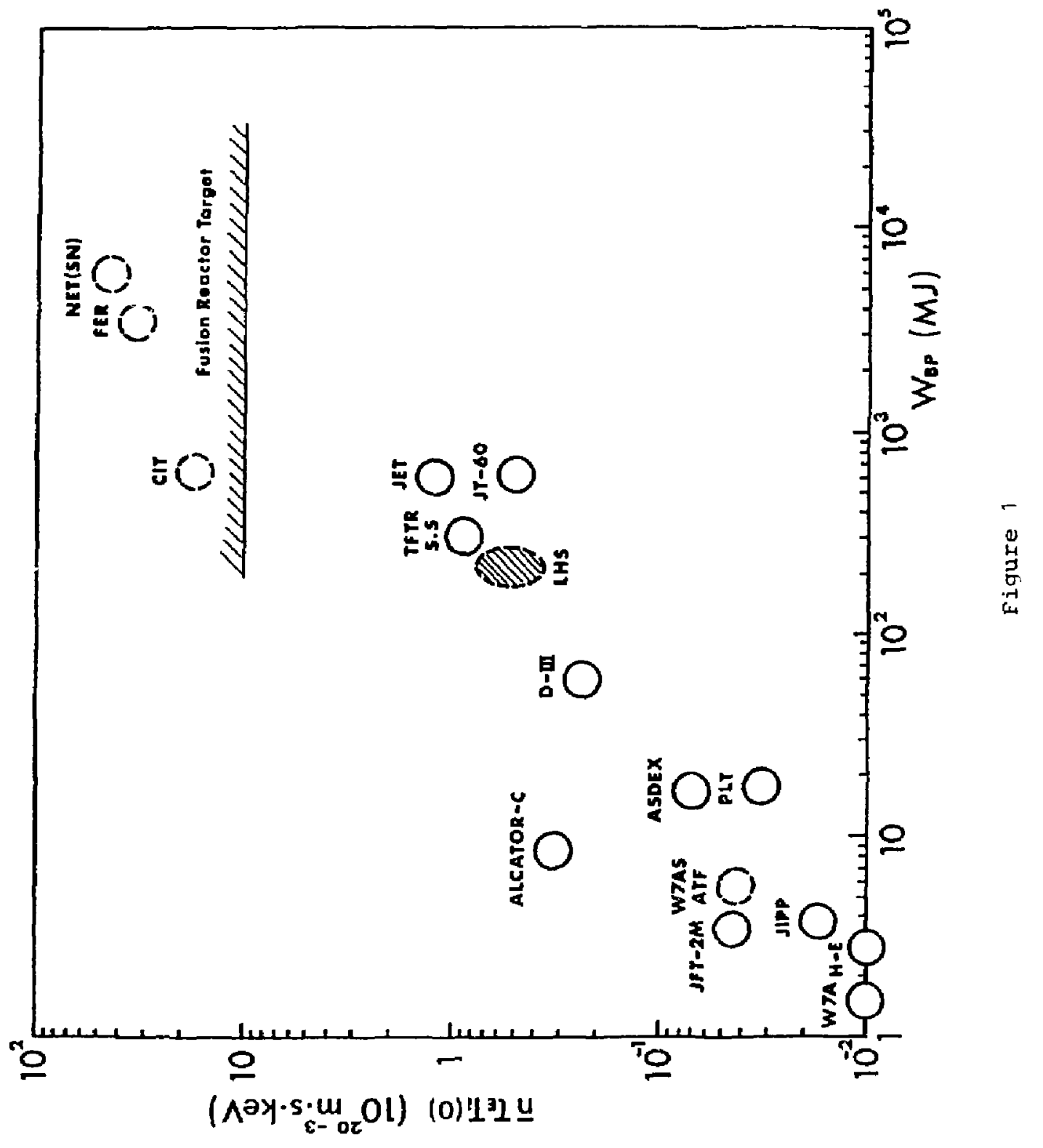




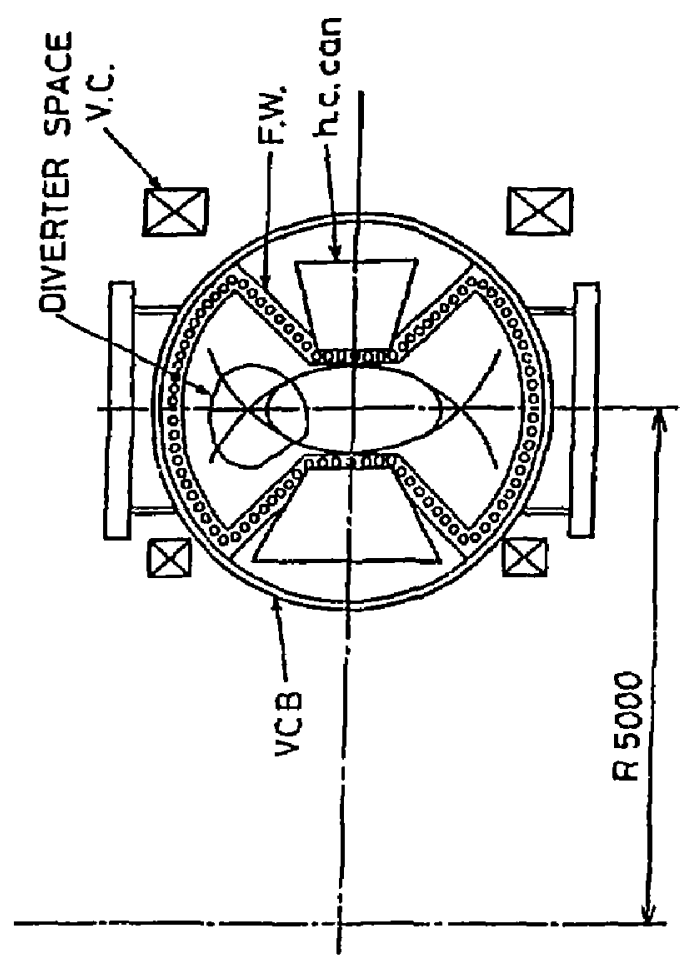

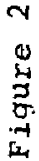

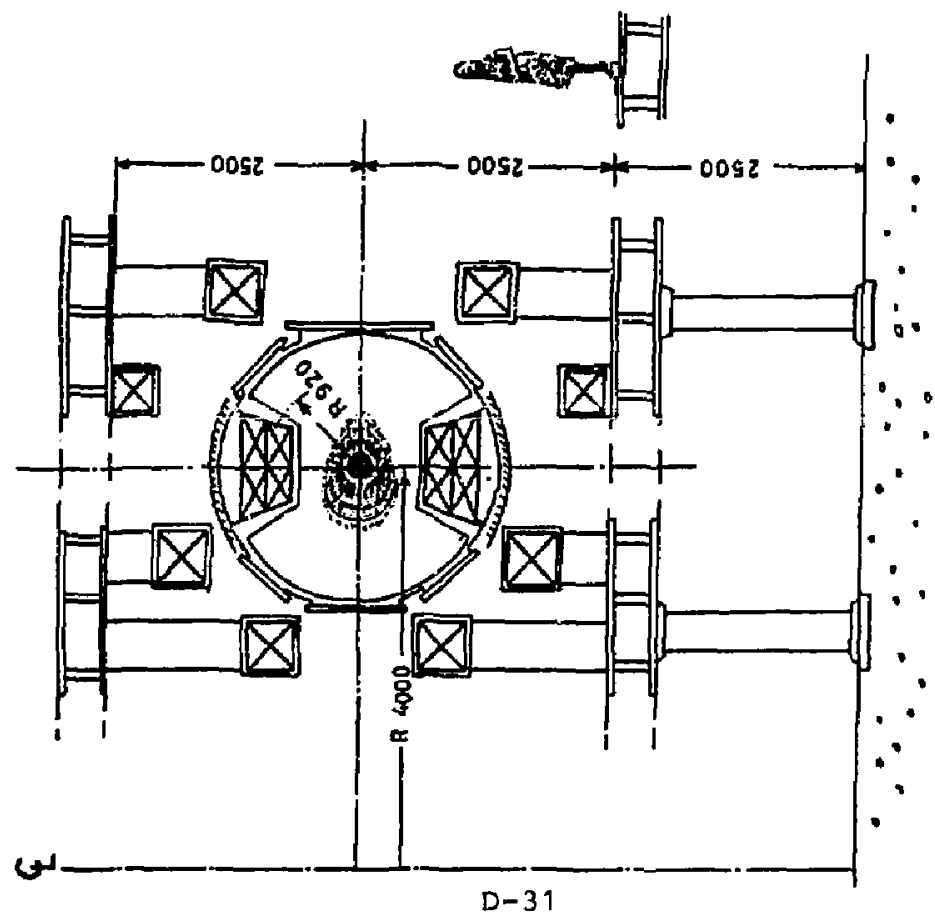




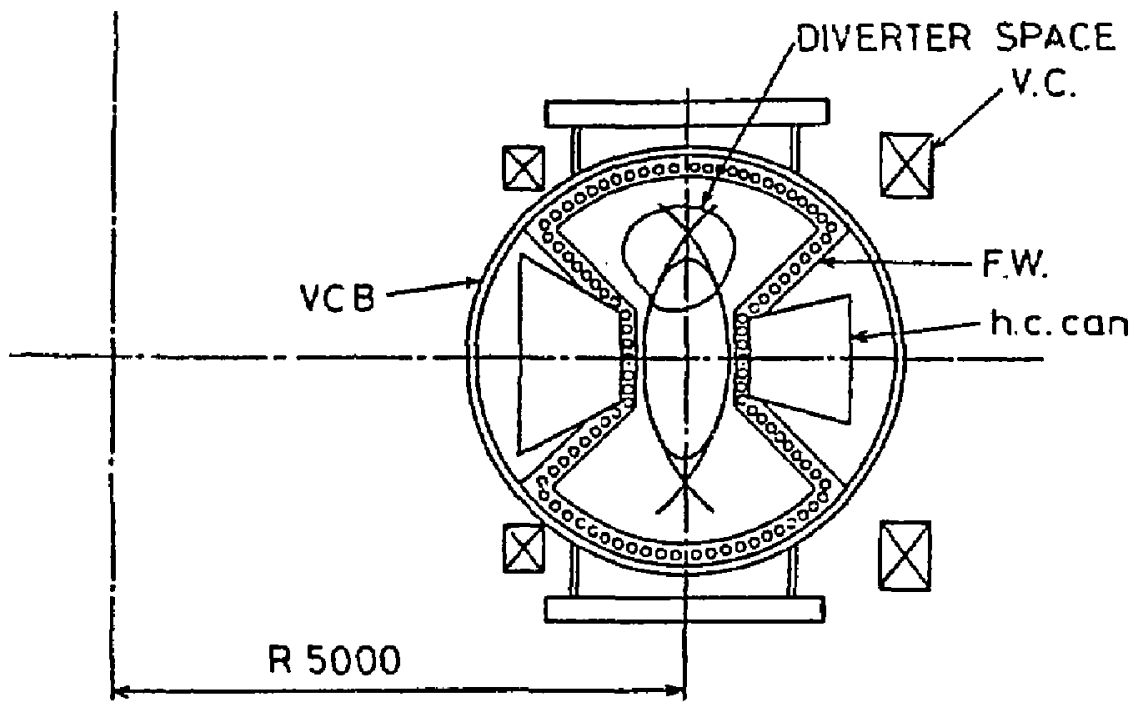

(a)
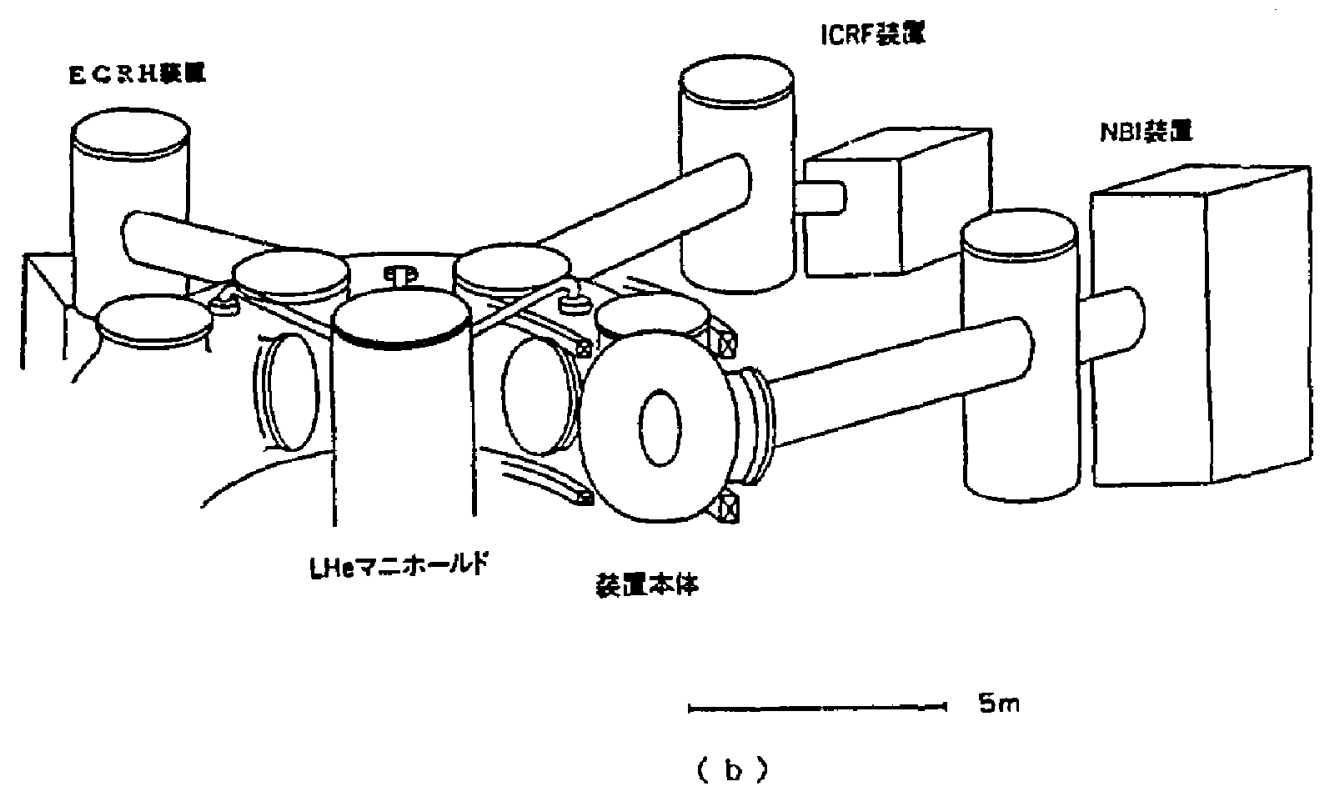


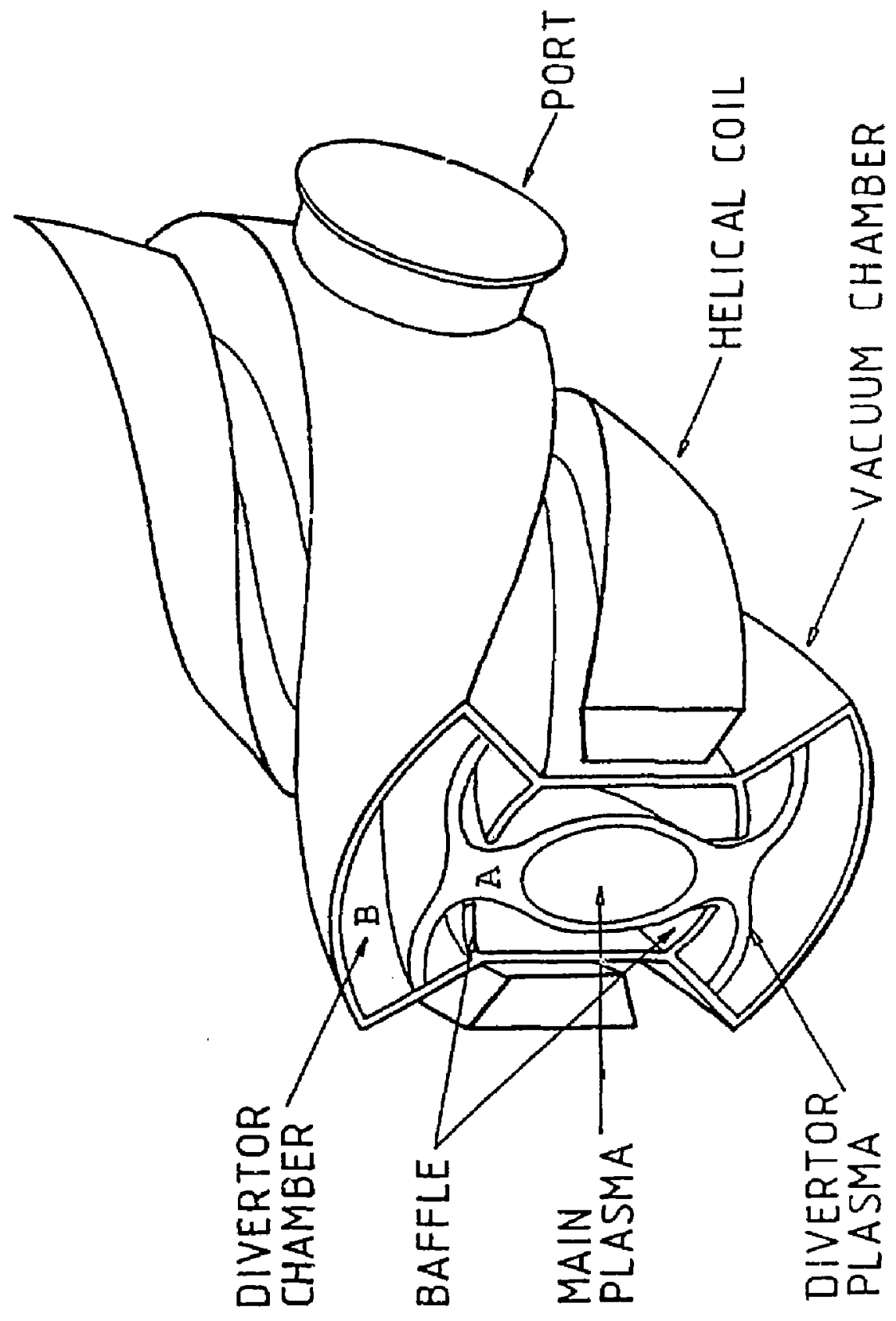




\section{U.S. PMI/HHF Laboratory Facilities}

The U.S. DOE supports laboratory facilities for the investigation of Plasma Materials Interactions and High Heat Flux components. Almost all of these facilities have some potential for addressing steady state issues in the PMI/HHF fields. The application of the facilities has been described in the other sections of this report that discuss the specific issues. The present capabilities, diagnostics, and types of experiments performed in each facility are described here. The laboratory facilities are summarized in Table $\mathrm{I}$, and described in detail in the following pages.

Table I. Summary of Existing U.S. PML/HHF Laboratory Facilities

\begin{tabular}{|c|c|c|}
\hline$\underline{\text { Name }}$ & Location & Status \\
\hline Plasma-Materials Test Facility (PMTF) & SNLA & Operating \\
\hline \multicolumn{3}{|l|}{-Electron Beam and Ion Beam } \\
\hline Plasma-Surface Interaction Experiment (PISCES) & UCLA & Operating \\
\hline \multicolumn{3}{|l|}{-High Density Plasma Source } \\
\hline Tritium Plasma Experiment (TPX) & SNLL & Operating \\
\hline \multicolumn{3}{|l|}{-Tritium Plasma Source } \\
\hline ORNL PMI Facilities & ORNL & Operating \\
\hline \multicolumn{3}{|l|}{-Outgassing and Glow Discharge Facilities } \\
\hline Hot-Cell Electron Beam Facility (HEBF) & HEDL & Under construction, \\
\hline -Neutron Irradiation and e-Beam Tests & & Components exist \\
\hline Continuous Current-Drive Tokamak (CCT) & UCLA & $\begin{array}{l}\text { Operating, funded for } \\
\text { current drive exps. }\end{array}$ \\
\hline Electromagnetic Forces (FELDX) & ANL & Not-operating \\
\hline
\end{tabular}




\section{Description of U.S. Facilities}

I. Plasma-Materials Tesi Facility (PMTF) at SNLA.

The PMTF facility utilizes electron beam (EBTS) and ion beam (IBTS) test facilities to evaluate the thermal and mechanical response of high heat flux components and materials to intense surface energy deposition. There are four categories of experiments performed with these facilities:

1. Thermal fatigue of materials under normal operating conditions in confinement devices.

2. Material response to off-normal heat loads.

3. Thermal hydraulic heat transfer studies.

4. Moderate scale component integrity tests.

The Electron Beam Test Stand (EBTS) system parameters are:

Electron Energy

Electron Current

Total Power

Power Density (maximum)

Pulse Duranion

Sample Size (maximum)

Cooling System

Control and Data Acquisition Sysıem
$30 \mathrm{kV}$

1 Ampere

$30 \mathrm{~kW}$

$100 \mathrm{~kW} / \mathrm{cm}^{2}$

50 msec to continuous

$30 \mathrm{~cm} \times 60 \mathrm{~cm}$

Water, 50GPM at 300PSI

LSI $11 / 73$

The standard operating mode for EBTS, shown in Fig. 1 , is to utilize a rastered beam 0.3 to $0.5 \mathrm{~cm}$ in diameter to heat an area of 1 to $100 \mathrm{~cm}^{2}$. The beam is normally rastered at $400 \mathrm{~Hz}$, and effective pulse lengths on the order of milliseconds can be achieved by the rapid sweep of the beam over the sample. Continuous heat deposition is likewise possible, and steady state heat deposition on materials can be investigated by EBTF. The diagnostics available at the facility include: 


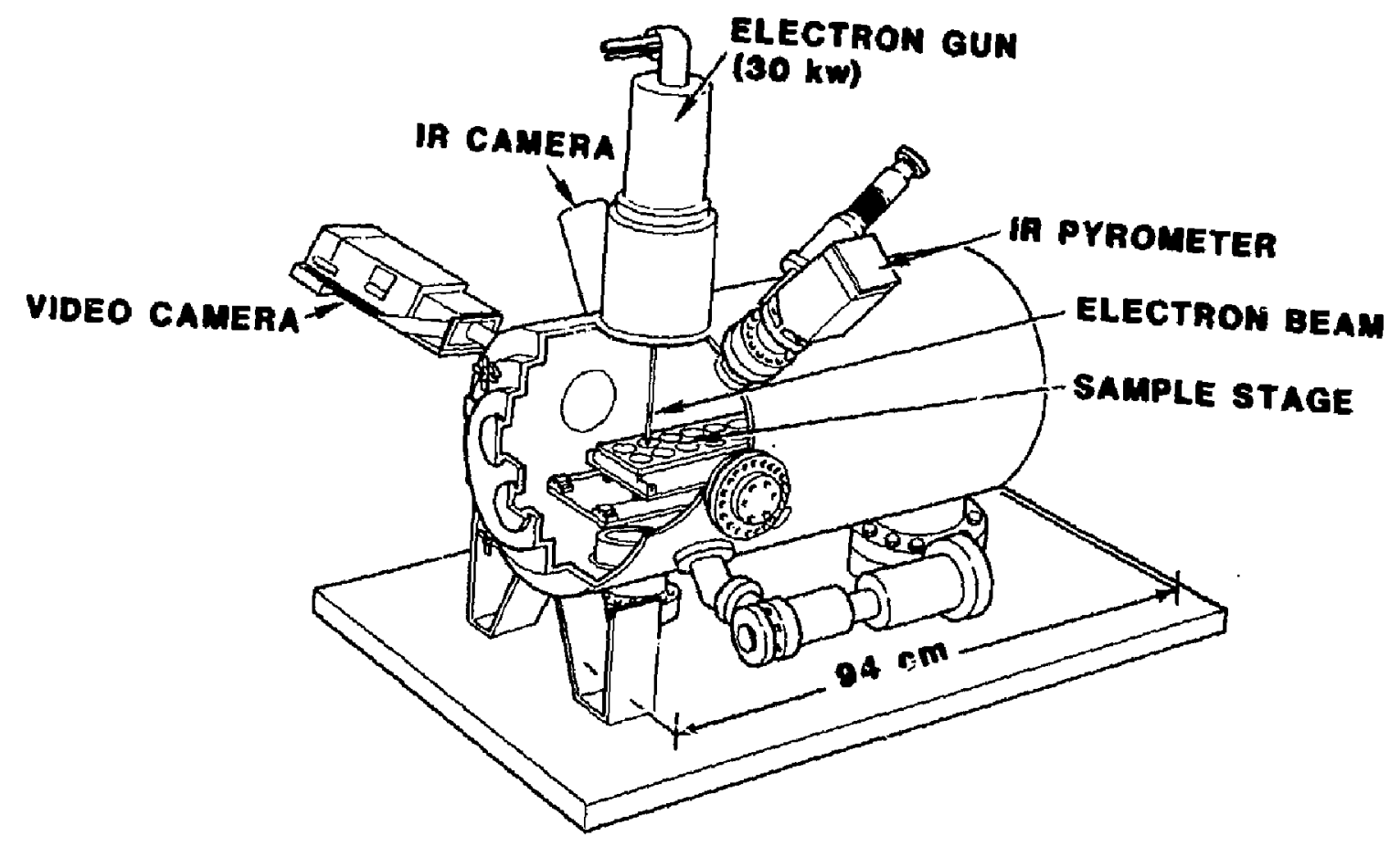

\section{Sandia National Laboratories}


Diagnostic

Infrared Pyrometer $\left(300-1300^{\circ} \mathrm{C}\right)$

Optical Pyrometer (1100-3300 0 C)

Infrared Camera $\left(20-3000^{\circ} \mathrm{C}\right)$

Imbedded Themocouple Arrays

Strain Gauges

RGA.

Water Cr'orimetry

Video Cameras

SEM

\section{Purpose}

Surface Temperature

Surface Temperature

Surface Temperature

Bulk Temperature

Mechanical Response

Outgassing

Power Deposition

Audio-Visual Observations

Metallurgical Studies

The Ion Beam Test Stand (IBTS) maximum system parameters are:

\section{Ion Energy \\ Ion Current \\ Total Power \\ Power Density (maximum)}

Pulse Duration

Sample Size (maximum)

Cooling System

Control and Data Acquisition System
$40 \mathrm{kV}$

20 Amperes

$800 \mathrm{~kW}$

$4 \mathrm{~kW} / \mathrm{cm}^{2}$ for $0.2 \mathrm{sec}$

$500 \mathrm{~W} / \mathrm{cm}^{2}$ for $12 \mathrm{sec}$

$\leq 12 \mathrm{sec}$

$1 \mathrm{~m}^{2}$

Water, 500GPM at $1000 \mathrm{PSI}$

Computer controlled

The Ion Beam Test Facility, shown in Fig.2, utilizes a rf plasma generator and multiple grid accelerator from the neutral beam developinent programs to generate a long pulse, high power ion beam. Large area, high heat flux components and structures can be tested in the facility during long pulse operation. While continuous operation of the ion beam is not planned at this time, steady state issues that can be studied during pule lengths of less than about $15 \mathrm{sec}$ can be investigated in IBTF. The diagnostics available at the facility are the same as EBTF. 

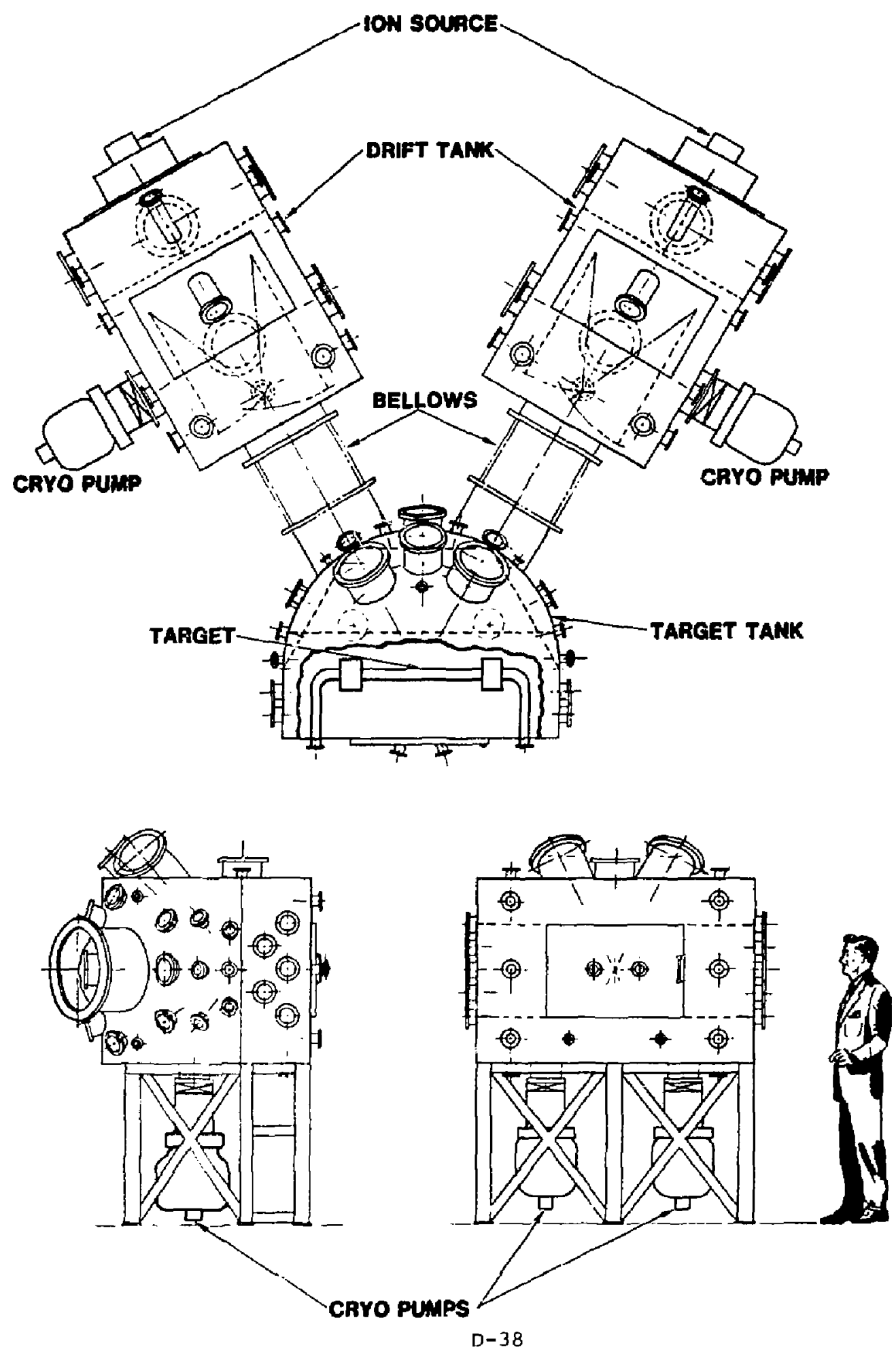
II. Plasma-Surface Interaction Experiment (PISCES) at UCLA.

The PISCES facility utilizes a high density, continuously operating plasma generator to study materials hehavior and plasma scrape-off-iayer physics. The experiments feature continuous bombardment of samples and components by plasmas with parameters representative of the scrape-off-layer in confinement devices. PISCES is dedicated to studying steady state issues of plasma materials interactions, and utilizes plasma fluxes and high fluences representative of major confinement machines such as tokamaks. There are three categories of experiments performed in PISCES:

1. Erosion and redeposition of PIC materials and components during continuous plasma bombardment.

2. Edge plasma simulation and impurity control experiments.

3. Experiments to expand the PMI data base and provide relationships for laboratory results to PMI results from confinement devices.

The PISCES system parameters are:

Gas Type

Operation Time

Flux Range

Plasma Density

Percentage Ionization

Electron Temperature

Ion Energy (bias)

Target Area

Heat Flux (maximum)

Magneric Field

Cooling System

Vacuum System

Control and Data Acquisition System
$\mathrm{H}, \mathrm{D}, \mathrm{He}, \mathrm{N}, \mathrm{Ar}$

Continuolss

$10^{17}-2 \times 10^{19} \mathrm{ions} / \mathrm{cm}^{2} \mathrm{sec}$

$10^{11}-10^{13} \mathrm{~cm}^{-3}$

$10-90 \%$

$3-30 \mathrm{eV}$

$30-500 \mathrm{eV}$

$50-400 \mathrm{~cm}^{2}$

$<500 \mathrm{~W} / \mathrm{cm}^{2}$

$200-1000$ gauss

Water or Air

$6000 \mathrm{~V} / \mathrm{sec}$ Turbo pumped

MicroVax II 
The facility features two plasma systems, PISCES A shown in Fig.3, and PISCES B shown in Fig.4, a UHV vacuum oven to bake and outgas the samples, and two UHV surface analysis stations. PISCES A is dedicated to edge plasma physics studies, and the UHV PISCES B system is dedicated to plasma wall interactions studies, although some crossover naturally occurs. The experiments in the laboratory focus on the erosion and redeposition of materials, impurity transport and control, edge plasma simulations, steady state issues for diagnostics and cooling, and expanding the PMI/HHF data base during high fluence plasma bombardment. The diagnostics available at the facility include:

\section{Diagnostic}

Oucical Multichannel Analyzer, 1.3m Spectrometer

Fast Scanning Langmuir Probes

Microwave Interferometer

Optical Pyrometer $\left(400-3000^{\circ} \mathrm{C}\right)$

Thermocouples

lonization gauges and baratrons

RGA

Water Calorimetry

Vacuum Oven with RGA

SEM

AES, SIMS

\section{Purpose}

Impurity concentration

Plasma Parameters

Plasma Density

Surface Temperature

Bulk Temperature

Gas Pressures

Partial Pressures

Power Deposition

Outgassíng composition

Surface Morphology

Surface Composition 


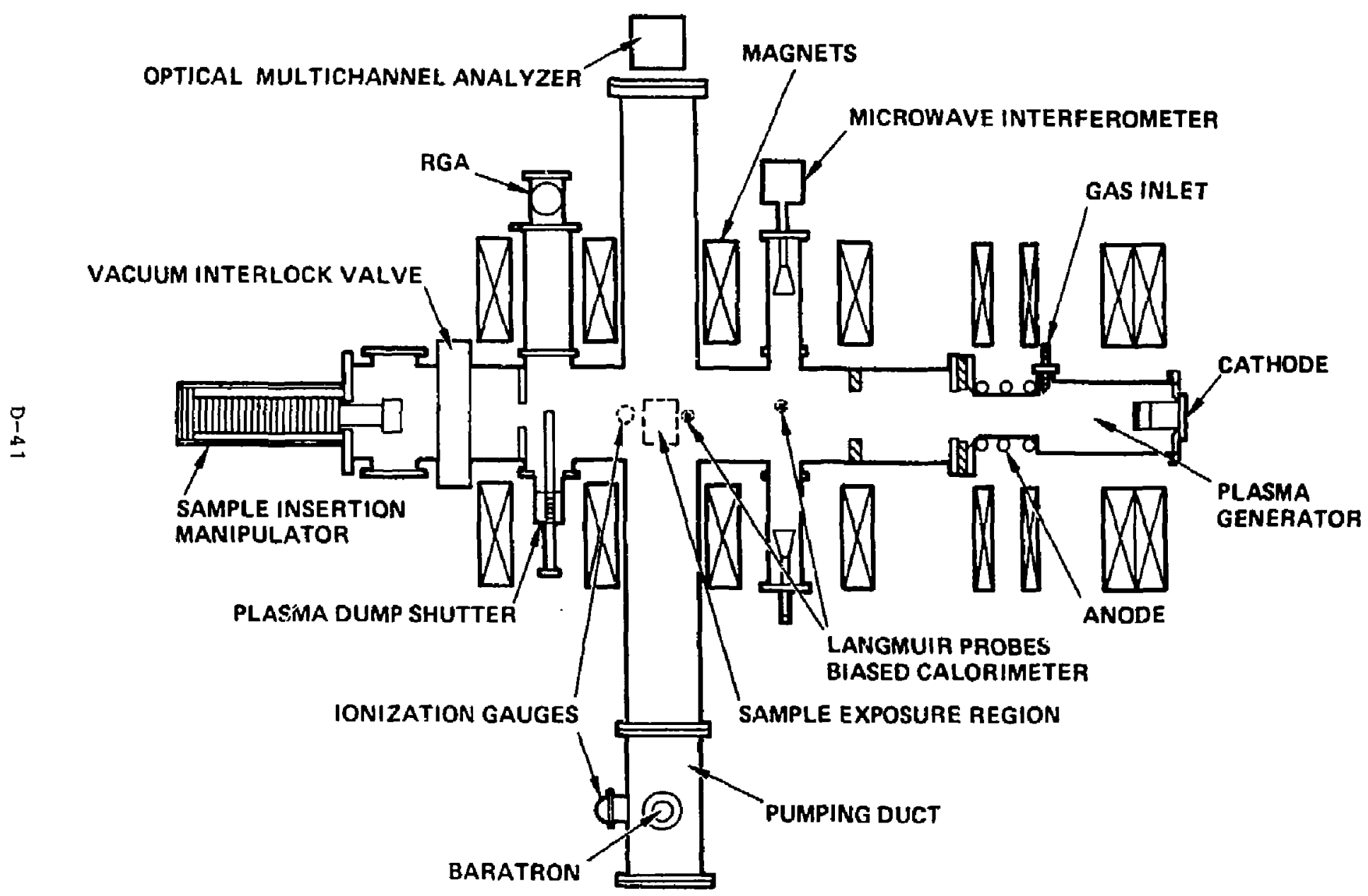




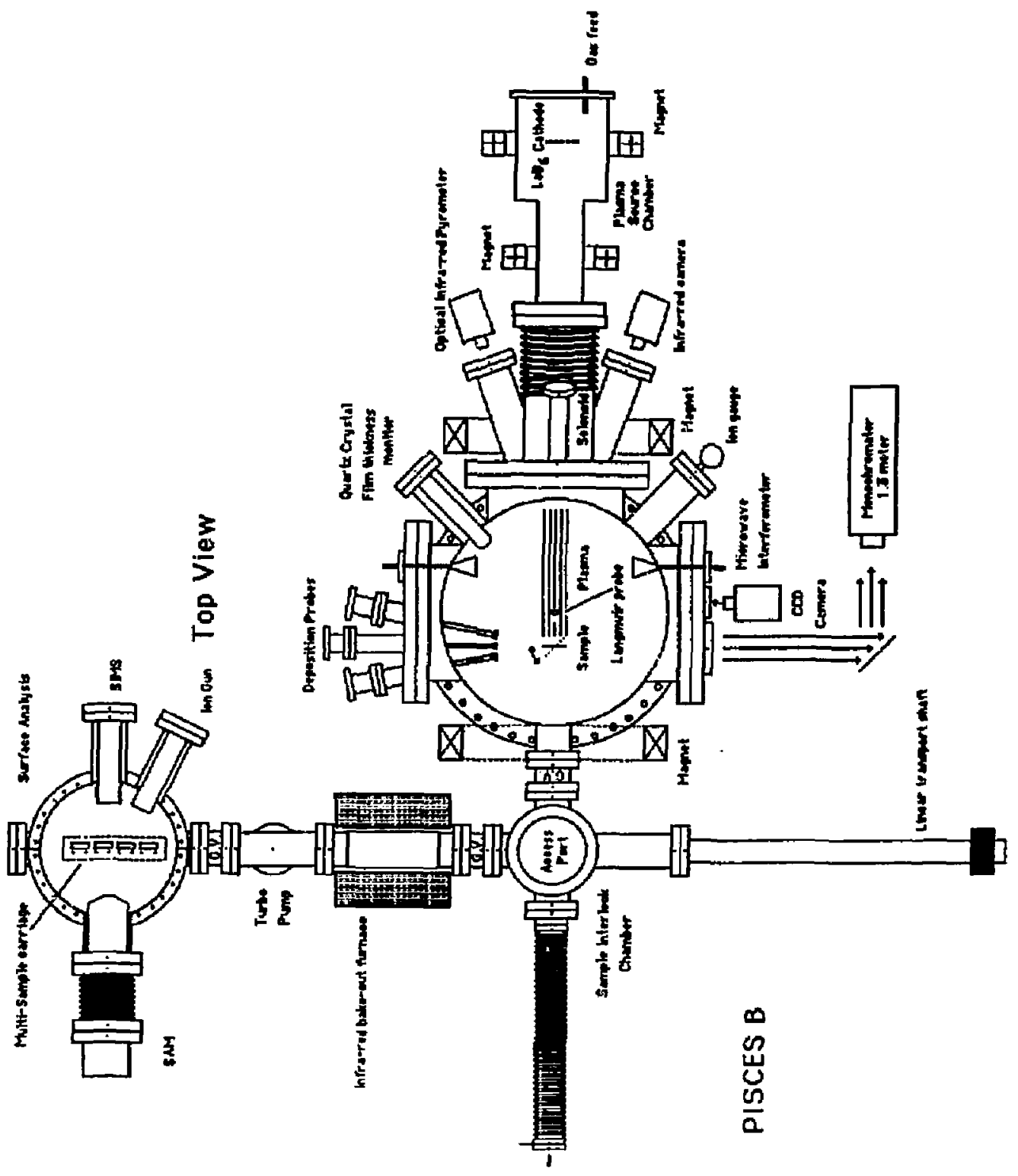




\section{Tritium Plasma Experiment (TPX) at SNLL}

The TPX facility, shown in Fig. 5, utilizes a low density, fi plasma generator to study plasma surface interactions and plasma driven permeation. The experiments feature continuous bombardment of samples by tritium plasmas or other working gases with traces of tritium. Steady state issues of permeation and other plasma material interactions can be studied in TPX. There are three categories of experiments performed in TPX:

1. Plasma driven permeation to provide a database for tritium permeation and inventory calculations.

2. Sputtering and erosion of materials by plasma bombardment.

3. Other experiments to expand the PMI data base.

The TPX system parameters are:

Gas Type

Plasma Sources

Operation Time

Flux Range

Plasma Density

Electron Temperature

Ion Energy (bias)

Target Area

The diagnostics available at the facility include:

Diagnostic

Langmuir Probes

Ionization gauges and manometers

RGA

Gas flow meter

Permeation Probe

In-situ AES

SEM

SIMS, RBS
$H, D, T$, others

RF and Glow Discharge

Continuous

$\leq 10^{17}$ ions $/ \mathrm{cm}^{2} \mathrm{sec}$

$<10^{11} \mathrm{~cm}^{-3}$

$<7 \mathrm{eV}$.

$15-300 \mathrm{eV}$

$10-20 \mathrm{~cm}^{2}$

\section{Purpose}

Plasma Parameters

Gas Pressures

Partial Pressures

Gas flow rate

Tritium permeation rates

Surface Composition

Surface Morphology

Surface Composition 


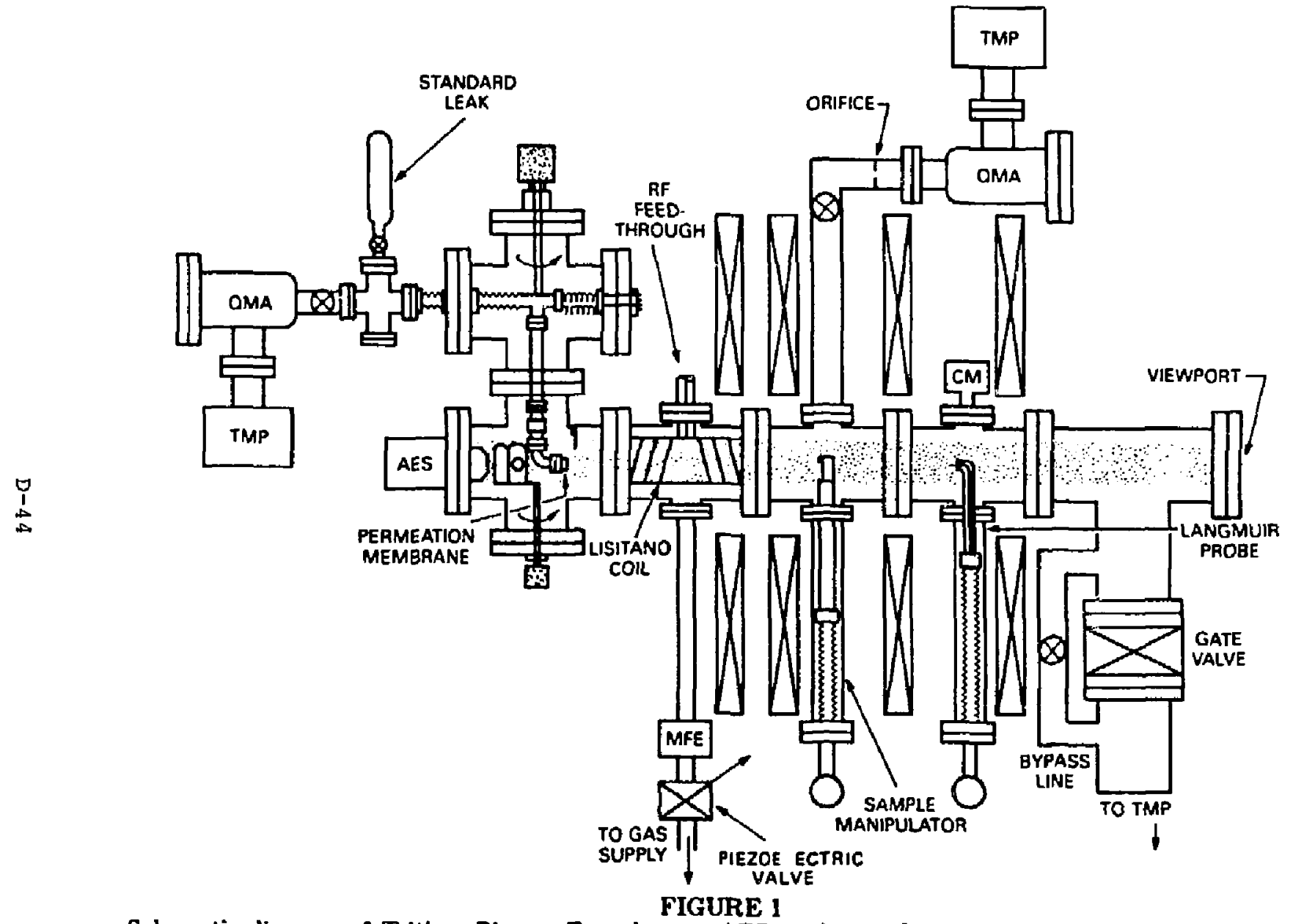

Scbematic diagram of Tritium Plasma Experiment. AES = Auger electron spectrometer, QMA = quadrupole mass analy zer, TMP = turbomolecular pump, MFE = molecular flow element, $\mathrm{CM}=$ capacitance manometer. 
IV. Hot-Cell Electron Beam Facility (HEBF) at HEDL

The HEBF facility, illustrated in Fig.6, will utilize an electron beam test facility which is remotely operated in a hot cell to evaluate the thermal and mechanical behavior of materials after exposure to neutron irradiation. High heat flux materials and coatings exposed to neutron bombardment in the FFTF MOTA 1-D reactor can be transported to the e-beam facility for basic material properties studies as a function of the material DPA and temperature. The major components of the system cxist, such as the e-beam equipment and hot cell, and the compiete facility is under construction. When operational, steady state issues of the characteristics of irradiated materials can be studied.

The HEBF system parameters are:

Electron Energy

Electron Current

Total Power

Power Density

Pulse Duration

Sample Size (maximum)

Cooling System

Hot cell operation

\section{$12-25 \mathrm{kV}$}

2.5 Ampere

$60 \mathrm{~kW}$

$0.3-60 \mathrm{~kW} / \mathrm{cm}^{2}$

$0.5 \mathrm{sec}$ to continuous

$1-200 \mathrm{~cm}^{2}$

Water, 20GPM at 120PSI 


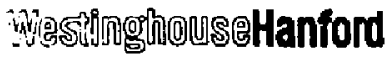

\section{POSTIRRADIATION HIGH}

\section{HEAT FLUX TEST FACILITY}

$p$
1
0

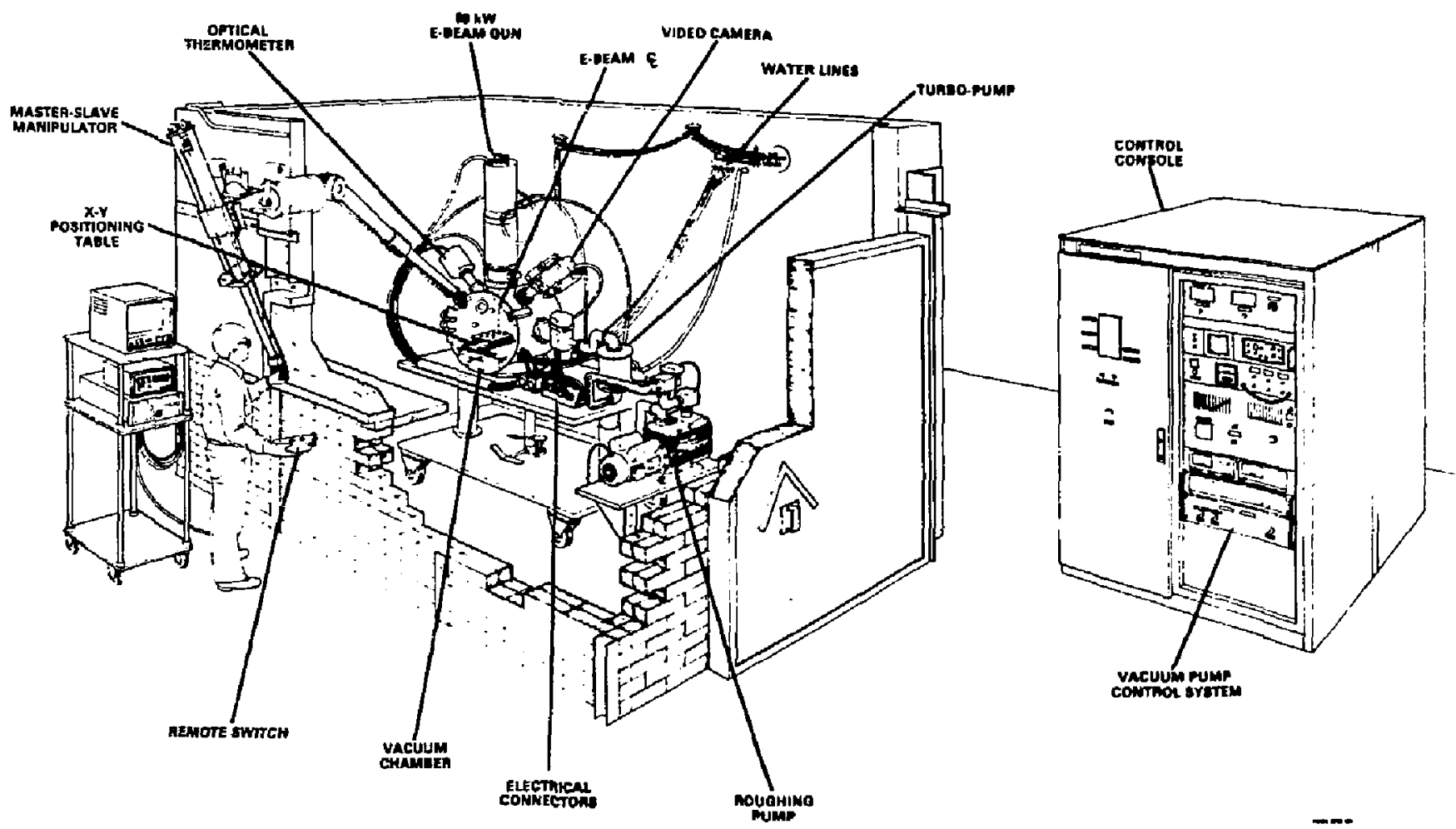




\section{ORNL PMI Facilities}

The Plasma Materials Interactions facilities at ORNL include a high temperature vacuum outgassing test stand and a hot cathode glow discharge plasma simulator. The outgassing facility is presently used for studies of the vacuum properties of graphite and other materials at temperature of up to $2000^{\circ} \mathrm{C}$. The plasma simulator investigates wall conditioning, recycling, retention, and carbon recycling. In addition, the Radio Frequency Test Facility (RFTF) at ORNL, which is funded for the development of large if components, has the potential for use in PML/HHF experiments. The parameters of the three facilities are:

\section{QUTGASSING FACILITY}

RF-induction heating (10kW) of large samples

Computer controlled sample temperature with thermocouple and Optical Pyrometer diagnostics Temperature controlled walls

RGA and ion gauge pressure diagnostics

\section{PLASMA SIMULATOR}

Plasma Source

Operation Time

Flux Range

Plasma Density

lon Energy (wall bias)

Graphite Liner and In-situ surface analysis
Hot cathode glow discharge

Continuous

$$
10^{14}-5 \times 10^{16} \text { ions } / \mathrm{cm}^{2} \mathrm{sec}
$$$$
<1011 \mathrm{~cm}^{-3}
$$

$0-500 \mathrm{eV}$

\section{RFTE}

Plasma Source

Operation Time

Plasma Density

Electron Temperature

Ion Energy

Target Area

Magnetic Field

Cooling System

$\mathrm{ECRH}(50 \mathrm{~kW}$ at $28 \mathrm{GHz})$

Continuous

$5 \times 10^{11} \mathrm{~cm}^{-3}$

$10 \mathrm{eV}$

unknown

$>1000 \mathrm{~cm}^{2}$

$3 \mathrm{~T}$

Water with $1 \mathrm{MW}$ removal 


\section{Continuous Current Tokamak (CCT) at UCLA.}

The CCT tokamak is a U.S. facility funded to investigate current drive, if coupling and heating, and wall conditioning during continuous operation. The machine is partially dedicated to PMI/HHF activity, which will address steady state plasma materials interactions in a tokamak. There are four categories of PMI/HHF related experiments performed in CCT:

1. Edge plasma properties, heat and particle flows during continuous operation.

2. Erosion and redeposition of PIC materials and components in a continuous tokamak plasma.

3. Impurity control experiments.

4. Benchmarking between "Open" continuous PMI simulators (e.g. PISCES) and tokamak experience (e.g. CCT).

The CCT parameters are:

Tokamak Plasma

Operation Time

$0.3 \mathrm{sec}$ at $\mathrm{I}_{\mathrm{p}}=100 \mathrm{kA}$

Continuous at $I_{p}=10 \mathrm{kA}$

Plasma Density

$5 \times 10^{12} \mathrm{~cm}^{-3}$

Temperature

$T_{i}=T_{e}=10-100 \mathrm{eV}$

Target Area

$>1000 \mathrm{~cm}^{2}$

Heat Flux (average)

$10-100 \mathrm{~W} / \mathrm{cm}^{2}$

Cooling System

Water

CCT is a large (PLT size), low density, low magnetic field tokamak capable of continuous operation. A view of CCT is sinown in Fig.7. While the program is funded for applied plasma physics studies, some fraction of the machine time is used to study the evolution of the edge plasma parameters and materials erosion and redeposition in steady state operation. As the facility matures, it is intended to pursue the development of impurity control systems and expand the PMI database for steady state tokamaks. 

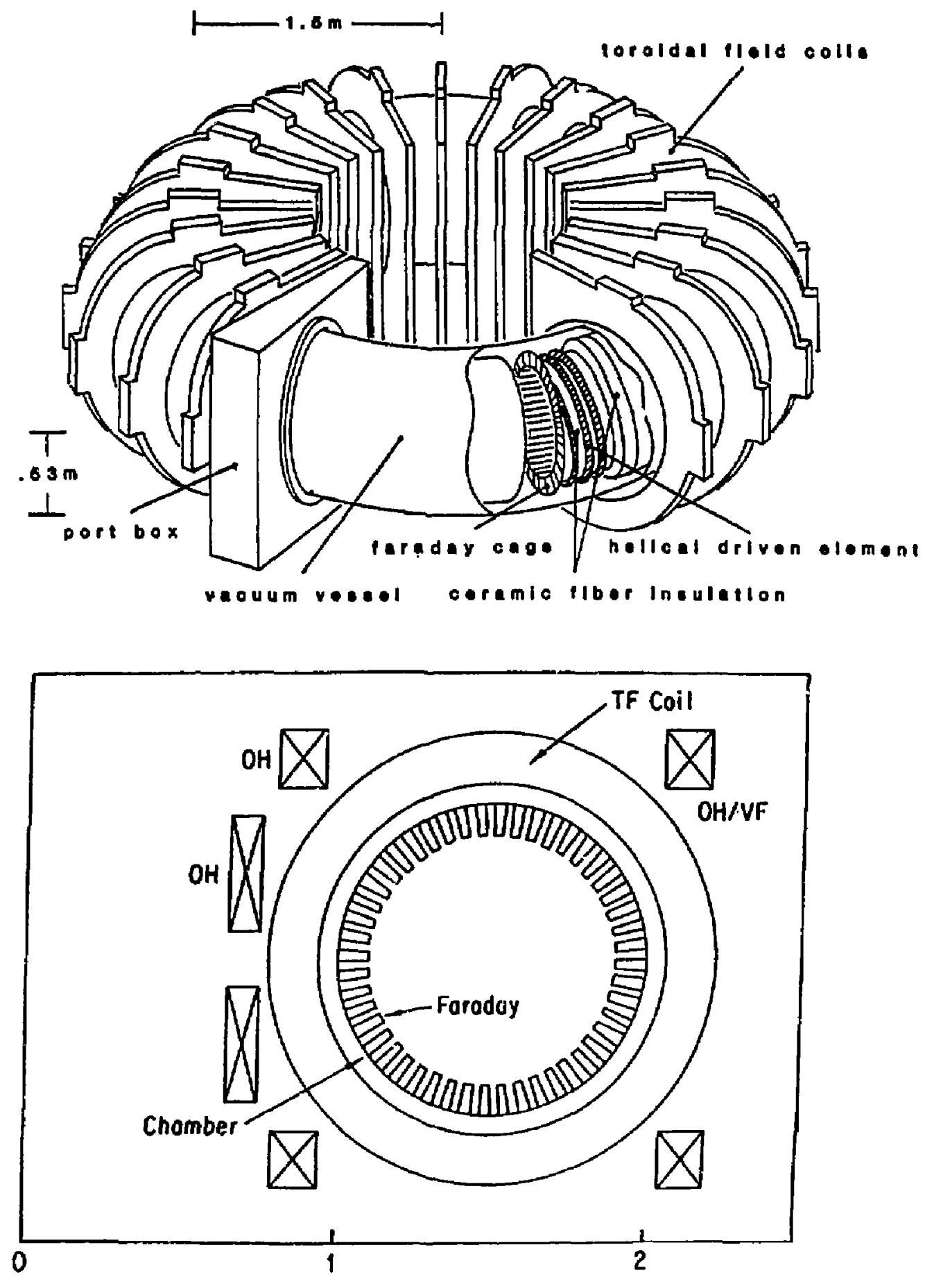

Mojor Radius (M) 
The diagnostics available at the CCT facility include:

Diagnostic

Standard Tokamak Diagnostics plus:

Spectrometers and Optical Multichannel Analyzers

Scanning Langmuir Probes

RGA
Purpose

Edge Impurity Concentration

Edge Plasma Parameters

Partial Pressures

Surface analysis (SEM, AES, SIMS) through PISCES collaborations. 